CHARGE

TRANSPORT IN

NANOSCALE

LATERAL

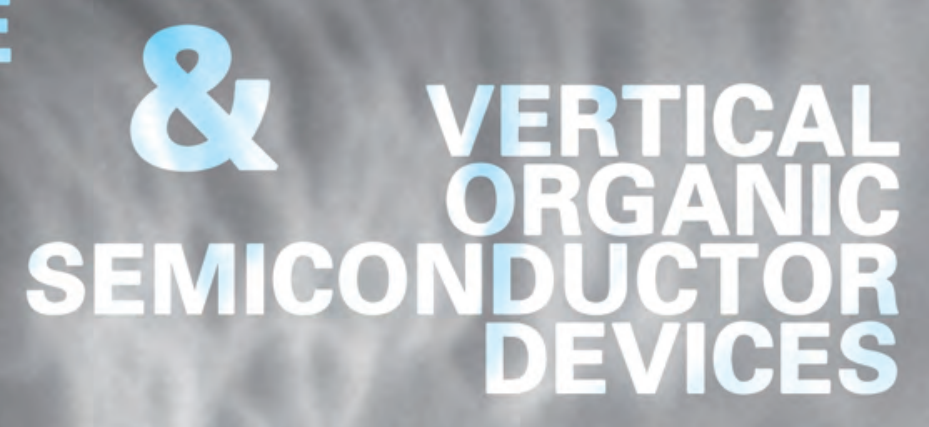


CHARGE TRANSPORT IN NANOSCALE LATERAL AND VERTICAL ORGANIC SEMICONDUCTOR DEVICES

Bojian Xu 
The research described within this thesis was carried out in the NanoElectronics Group at the MESA+ Institute for Nanotechnology at the University of Twente, Enschede, The Netherlands. The NWO-nano (STW) program, grant no. 11470, and the China Scholarship Council program, grant no. 201206090154 financially supported this research.

\section{UNIVERSITY OF TWENTE. MESA+}
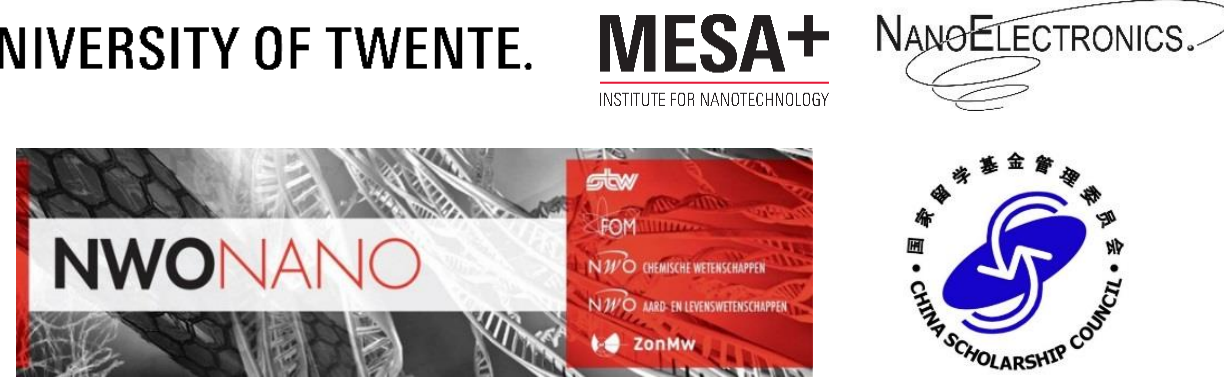

\section{Thesis committee members}

\section{Chairman \& secretary:}

Prof.dr. P.M.G. Apers

University of Twente

\section{Promotor:}

Prof.dr.ir. W.G. van der Wiel

University of Twente

\section{Other members:}

Prof.dr. P.A. Bobbert

University of Twente

Prof.dr.ir. G. Koster

University of Twente

Dr.ir. M.P. de Jong

University of Twente

Prof.dr. P.W.M. Blom

Max Planck Institute for

Polymer Research, Mainz

Prof.dr. B.J. Ravoo

University of Münster

Title: Charge transport in nanoscale lateral and vertical organic semiconductor devices Author: Bojian Xu

Cover design: Ximing Fu

Copyright (C) 2017 by Bojian Xu, Enschede, The Netherlands.

Printed by Gildeprint, Enschede, The Netherlands, 2017.

ISBN: 978-90-365-4286-9

DOI: $10.3990 / 1.9789036542869$ 


\title{
CHARGE TRANSPORT IN NANOSCALE LATERAL AND VERTICAL ORGANIC SEMICONDUCTOR DEVICES
}

\author{
DISSERTATION
}

\author{
to obtain \\ the degree of doctor at the University of Twente, \\ on the authority of the rector magnificus, \\ prof.dr. T.T.M. Palstra,
} on account of the decision of the graduation committee, to be publicly defended on Friday March $10^{\text {th }} 2017$ at 14.45

by

\section{Bojian Xu}

born on March $14^{\text {th }}, 1987$

in Jiangsu, China 
This dissertation has been approved by:

\section{Promotor:}

Prof.dr.ir. W.G. van der Wiel 
This thesis is dedicated to my family. 



\section{Contents}

1 Introduction and motivation ............................................................................ 1

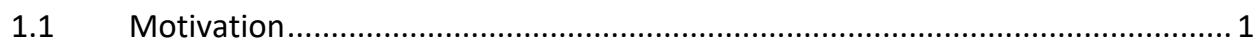

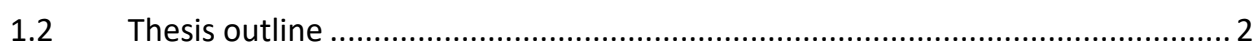

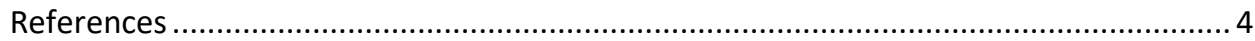

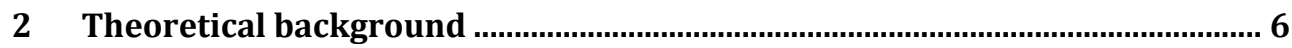

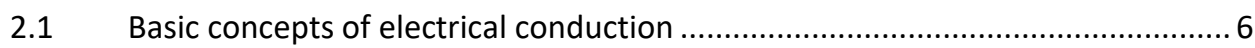

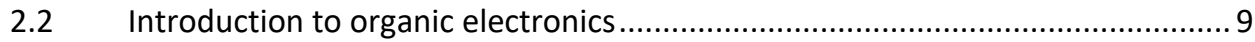

2.2.1 Molecular orbitals, energy bands/levels, band conduction and hopping

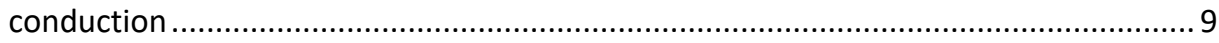

2.2.2 Why organic materials? .......................................................................... 13

2.2.3 Subfields of organic electronics ............................................................. 14

$2.3 \quad$ Organic field-effect transistors.................................................................. 16

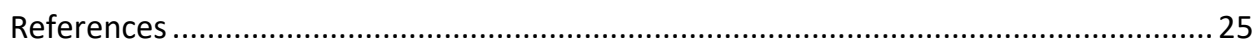

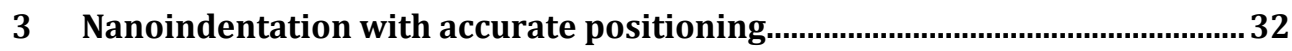

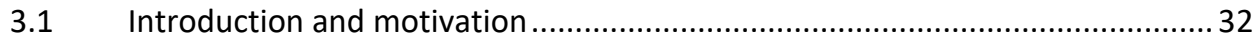

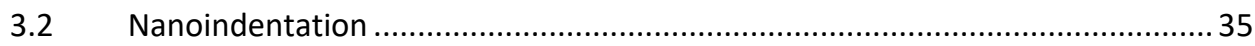

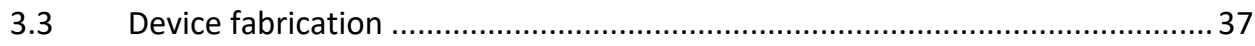

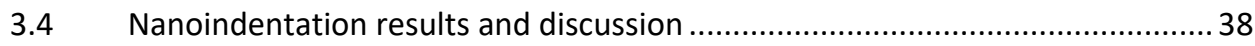

3.4.1 Nanoindentation using conventional working modes of the Veeco Dimension

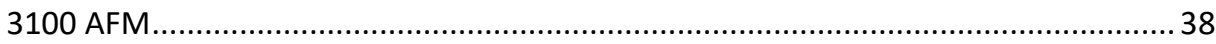

3.4.2 Nanoindentation with accurate positioning using the "point-and-shoot"

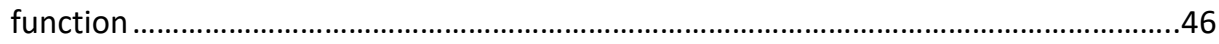

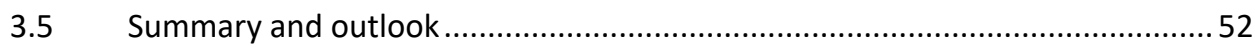

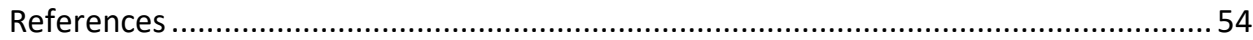

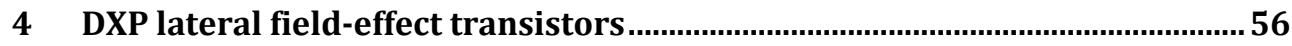

$4.1 \quad$ Introduction and motivation .................................................................... 56

4.2 Experiments and results............................................................................. 56

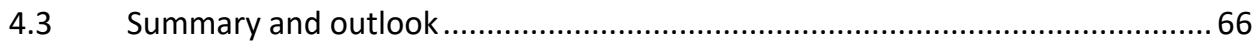


5 Charge transport in nanoscale vertical P3HT pillar devices ...........................69

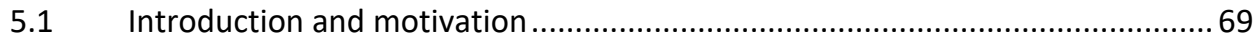

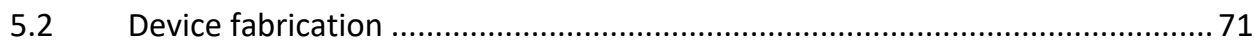

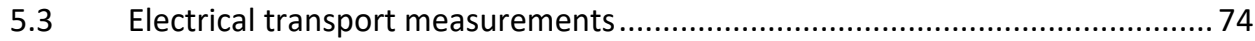

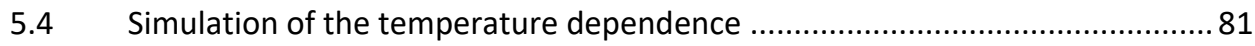

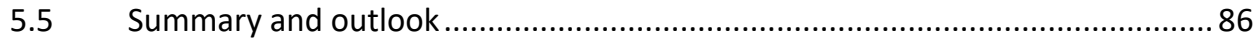

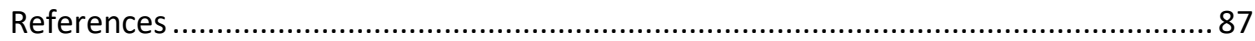

6 Vertical P3HT field-effect transistors............................................................... 91

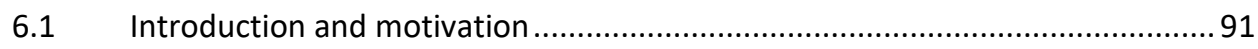

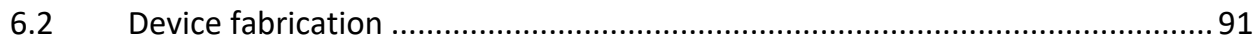

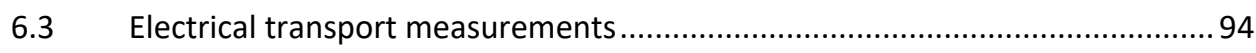

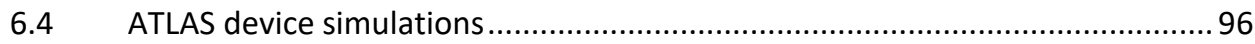

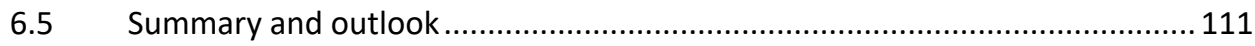

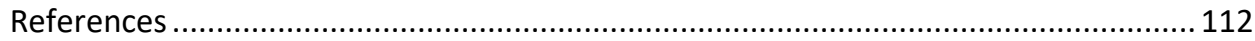

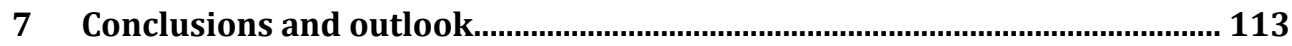

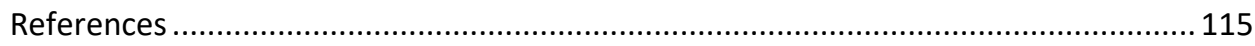

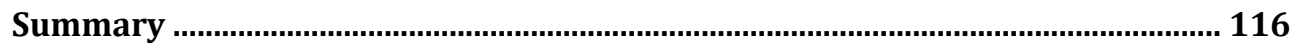

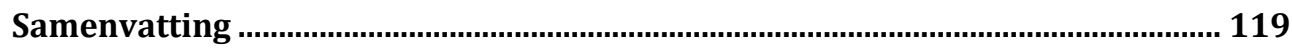

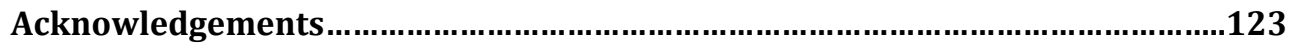




\section{Chapter 1}

\section{Introduction and motivation}

\subsection{Motivation}

Organic semiconductor materials are being intensely studied because of their extensive application in low-cost [1], flexible [2], biocompatible [3] electronic and spintronics [4] devices. Examples include organic field-effect transistors (OFETs) [5], organic light-emitting diodes (OLEDs) [6], and organic spin valves (OSVs) [7]. In this thesis research, we have investigated charge transport in organic semiconductor devices. Specifically, we focused on two organic materials, N, N'-bis(2,6-dimethylphenyl)-perylene-3,4,9,10-tetracarboxylic diimide (DXP) and poly(3-hexylthiophene) (P3HT), in different device configurations.

DXP is a molecular organic semiconductor. It can be assembled into molecular wires when the molecules are inserted in the nanopores of zeolite $L$ crystals [8, 9]. Electrical transport in the molecular wires is expected to have distinctive properties due to the spatial constriction of the molecular wires [10]. To investigate the electrical transport of the DXPloaded zeolite $L$ crystals in multiple experimental instruments, we planned to embed the crystals into devices. Hence, we designed a device fabrication process based on the nanoindentation technique using atomic force microscope (AFM). In the fabrication process, a critical step was to execute the nanoindentation precisely and only on top of the crystals with 150 - $300 \mathrm{~nm}$ diameter. Therefore, it was necessary to carefully verify the positioning properties of the nanoindentation technique.

Lateral field-effect transistors are suitable for investigation of the electrical transport properties in organic semiconductors [11]. In addition, one can integrate on-chip coplanar waveguides so that magnetic resonance experiments can be performed to study spin dynamics $[12,13]$. By investigating lateral FETs with channel lengths similar to the lengths (sub-100 to hundreds of $\mathrm{nm}$ ) of the molecular wires in the zeolites mentioned above, we could compare results between the lateral devices and the DXP-loaded crystals. Hence, apart from the DXP-molecular-wire devices, we also investigated the charge transport properties of the DXP lateral field-effect transistors with $100 \mathrm{~nm}$ channel length.

There are more reasons to investigate OFETs with a short channel length. It has been reported that decreasing the channel length can increase the cut-off frequency of devices $[14,15]$. At small spacing between electrodes, the electric field at low voltage can still be very high. Therefore, the short-channel OFETs can operate at low voltages, while 
maintaining sizeable current densities, which is beneficial for implementation in low-power electronic devices $[16,17]$. In lateral OFET devices, as in the DXP lateral devices, nanolithography techniques are needed to fabricate short spacing electrodes. However, in a vertical configuration, an organic film is sandwiched between two metallic contacts, and the channel length is defined by the film thickness which is well controllable down to a few $\mathrm{nm}$. Nevertheless, fabricating top contacts on thin organic films and patterning organic layers are not straightforward. For the top contacting, the main difficulty is to nondestructively deposit top contacts on the organic film without damaging or introducing contaminants or extra layers. As for the patterning, chemicals used in standard nanolithography, e.g., photolithography and electron beam lithography, and lift-off processes can affect the organic films $[18,19]$. So, we designed a fabrication method to realize vertical organic devices with short junction length. $\mathrm{P} 3 \mathrm{HT}$ is a widely-investigated organic semiconductor. The polymer chains of P3HT can orderly stack together and thus form lamella structures in microcrystalline domains [20, 21]. The order of the microstructure leads to moderately good electrical mobility of P3HT. P3HT can form continuous and flat thin films by spin-coating. Hence, we first fabricated two-terminal P3HT devices in the vertical configuration, and investigated the charge transport in these devices. Then, to add gate control of the charge transport in the two-terminal devices, we fabricated and investigated the charge transport in three-terminal P3HT devices. A surrounding gate electrode including conformal gate dielectric layer was fabricated around the two-terminal device.

\subsection{Thesis outline}

In Chapter 2, we briefly introduce the theoretical background related to the experimental and numerical simulation research in this thesis.

The fabrication method based on the nanoindentation technique and the nanoindentation results are reported in Chapter 3. Two atomic force microscopes, the Veeco Dimension 3100 AFM and the Bruker Dimension Icon AFM were used to perform the nanoindentation on the DXP-loaded zeolite $L$ crystals and test samples. We analyzed the nanoindentation results of the two AFMs, and demonstrated a reliable technique for nanoindentation on top of the crystals. Test devices were fabricated and measured to verify the fabrication method.

The results on DXP lateral field-effect transistors are described in Chapter 4. We first describe the details of the device fabrication and electrical transport measurements. Two solution processes for the DXP deposition, spin-coating and drop-casting were compared. Two electrode configurations, single-gap electrodes and interdigitated electrodes were 
investigated. Electromigration and air degradation during the electrical transport measurements are also observed. Measurement results, including light response, hysteresis, output and transfer characteristics, are summarized and discussed.

The results of the vertical P3HT devices are reported in Chapter 5 and Chapter 6 . In Chapter 5 , we focus on two-terminal P3HT vertical pillar devices. The fabrication process is briefly described. The room-temperature electrical transport measurement results and the temperature dependence are discussed. Numerical simulations based on the GaussianDOS-improved drift-diffusion model were also performed. The results are summarized and discussed.

In Chapter 6, we research further on gated P3HT vertical pillar devices. The fabrication process is concisely reported. Experimental output and transfer characteristics of the devices are summarized and discussed. Device simulations were executed as well using the commercial software Silvaco ATLAS.

Finally, the conclusions of the thesis and an outlook for future research are summarized in Chapter 7. 


\section{References}

1. Forrest, S.R., The path to ubiquitous and low-cost organic electronic appliances on plastic. Nature, 2004. 428(6986): p. 911-918.

2. Lewis, J., Material challenge for flexible organic devices. Materials today, 2006. 9(4): p. 38-45.

3. Irimia-Vladu, M., et al., Biocompatible and Biodegradable Materials for Organic Field-Effect Transistors. Advanced Functional Materials, 2010. 20(23): p. 40694076.

4. Naber, W., S. Faez, and W. Van Der Wiel, Organic spintronics. Journal of Physics D: Applied Physics, 2007. 40(12): p. R205.

5. Klauk, H., Organic electronics: materials, manufacturing, and applications. 2006: John Wiley \& Sons.

6. Geffroy, B., P. Le Roy, and C. Prat, Organic light-emitting diode (OLED) technology: materials, devices and display technologies. Polymer International, 2006. 55(6): p. 572-582.

7. Wang, F. and Z.V. Vardeny, Recent advances in organic spin-valve devices. Synthetic Metals, 2010. 160(3): p. 210-215.

8. Ruiz, A.Z., et al., Synthesis of zeolite L. Tuning size and morphology. Monatshefte für Chemie/Chemical Monthly, 2005. 136(1): p. 77-89.

9. Huber, S. and G. Calzaferri, Energy transfer from dye-zeolite L antenna crystals to bulk silicon. ChemPhysChem, 2004. 5(2): p. 239-242.

10. Choi, S.H., B. Kim, and C.D. Frisbie, Electrical resistance of long conjugated molecular wires. Science, 2008. 320(5882): p. 1482-1486.

11. Kokil, A., K. Yang, and J. Kumar, Techniques for characterization of charge carrier mobility in organic semiconductors. Journal of Polymer Science Part B: Polymer Physics, 2012. 50(15): p. 1130-1144.

12. Dehollain, J., et al., Nanoscale broadband transmission lines for spin qubit control. Nanotechnology, 2012. 24(1): p. 015202.

13. Behrends, J., et al., Bipolaron formation in organic solar cells observed by pulsed electrically detected magnetic resonance. Physical review letters, 2010. 105(17): p. 176601.

14. Klauk, H., U. Zschieschang, and M. Halik, Low-voltage organic thin-film transistors with large transconductance. Journal of Applied Physics, 2007. 102(7): p. 074514.

15. Kitamura, M. and Y. Arakawa, High current-gain cutoff frequencies above $10 \mathrm{MHz}$ in n-channel C60 and p-channel pentacene thin-film transistors. Japanese Journal of Applied Physics, 2011. 50(1S2): p. 01BC01.

16. Sawabe, K., et al., Current-Confinement Structure and Extremely High Current Density in Organic Light-Emitting Transistors. Advanced Materials, 2012. 24(46): p. 6141-6146.

17. Fischer, A., et al., An all C 60 vertical transistor for high frequency and high current density applications. Applied Physics Letters, 2012. 101(21): p. 213303. 
18. Jia, H., et al., Patterning effects on poly (3-hexy/thiophene) organic thin film transistors using photolithographic processes. Organic electronics, 2007. 8(1): p. 44-50.

19. Gundlach, D., et al., Solvent-induced phase transition in thermally evaporated pentacene films. Applied Physics Letters, 1999. 74(22): p. 3302-3304.

20. Sirringhaus, H., et al., Two-dimensional charge transport in self-organized, highmobility conjugated polymers. Nature, 1999. 401(6754): p. 685-688.

21. Northrup, J.E., Atomic and electronic structure of polymer organic semiconductors: P3HT, PQT, and PBTTT. Physical Review B, 2007. 76(24): p. 245202. 


\section{Chapter 2}

\section{Theoretical background}

In this chapter we provide a brief overview of the underlying concepts of organic electronics as far as they are relevant for the experiments being described in the following chapters. We refer to the relevant literature where possible. Organic electronics is a research field focusing on the electronic properties of organic materials, and the application of those materials [1, 2]. Organic electronics usually refers to the electron transport properties of organic materials [3], although sometimes it also involves other aspects such as photonics $[4,5]$ and magnetism [6-8]. In this thesis, carrier transport in organic semiconductor devices is studied. Specifically, we investigated charge transport of two organic materials, DXP and $\mathrm{P} 3 \mathrm{HT}$, in different device configurations.

\subsection{Basic concepts of charge transport}

\section{A. Orbital overlap and delocalization}

In electrical 'conductors' electrons are free to move. In 'insulators' on the contrary, electrons are bound tightly to individual atoms [9]. For electrical conduction it is crucial that the charge carriers are delocalized inside the material. The delocalization results from the overlap of electronic orbitals $[10,11]$ when the atoms or molecules are sufficiently close to each other [12], depending on how atoms and molecules are organized.

In a quantum-mechanical description, the electronic orbitals can be derived from the electronic wave functions from which the probability density of finding electrons at a certain place is derived. The electron orbitals are usually visualized as electron "clouds", which schematically show the distribution of the probability density. In metals, a large number of outer shell electronic orbitals overlap to such an extent that those electrons are delocalized over the whole material [11].

To quantitatively describe the electronic properties of solid materials, it is very instructive to look at the reciprocal space (or momentum space), and study the band/energy-level structure of materials. 


\section{B. Energy band theory}

The electronic properties of solid materials can be explained or predicted by energy band theory. The energy bands are formed by dispersion/broadening of atomic (molecular) energy levels because of interatomic (intermolecular) interactions when a large number of atoms (molecules) are packed together periodically and considering their coulomb interactions. The band structure is derived from the energy dispersion relation, which is calculated by solving the Schrödinger equation for electrons in the solid. Based on the band structure, materials can be classified into insulators, semiconductors and metals, see Fig. 2.1.

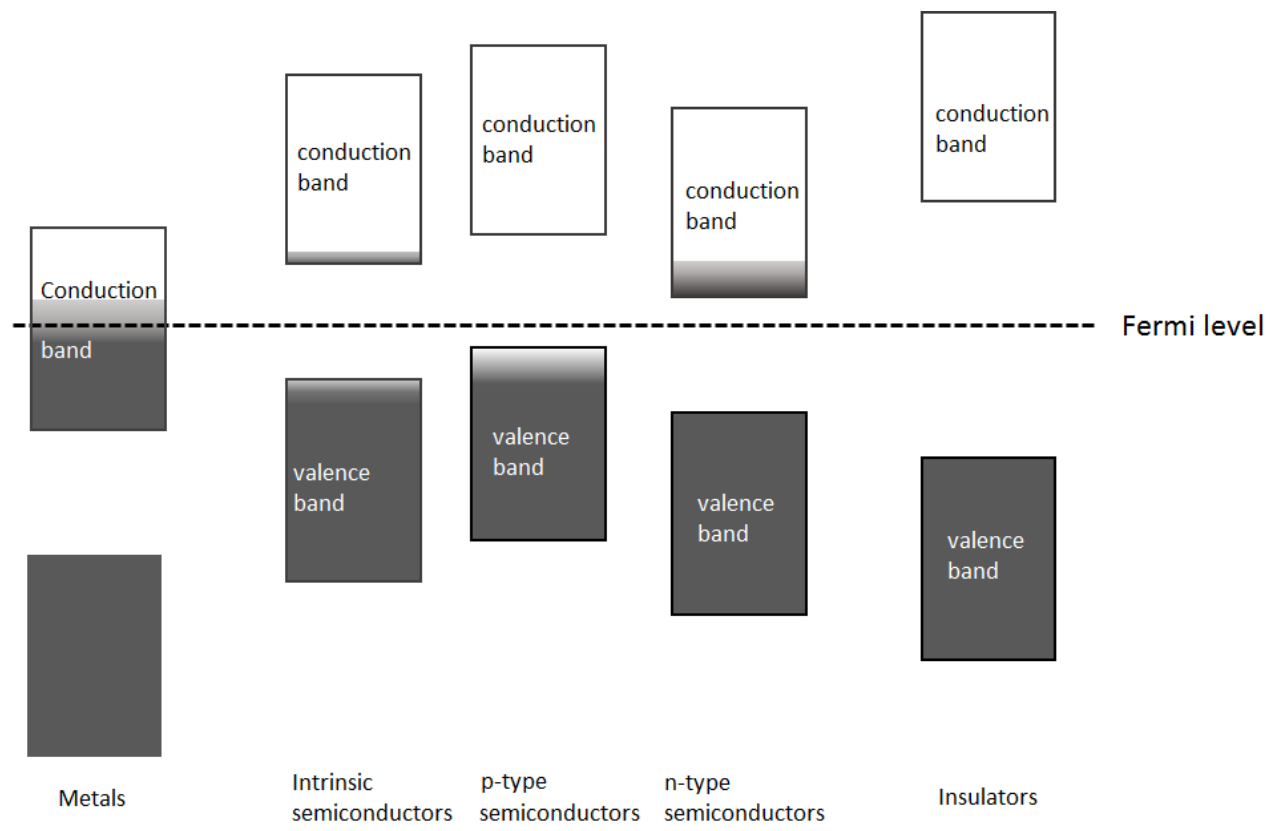

Figure 2.1. Schematic energy band diagrams of metals, semiconductors and insulators at finite temperature. At the Fermi level the electron occupation probability is $1 / 2$ for all temperatures. Finite temperature leads to excitation of electrons from below the Fermi level to energy levels above [13].

When the valence band is fully occupied by electrons and the conduction band is empty, there are no empty states for the valence electrons. Therefore, those electrons cannot delocalize, resulting in an electrical insulator. When there are electrons in the conduction band, or empty states, or 'holes', in the valence band, electrons can scatter into unoccupied states and delocalize [13]. 
The conduction and valence bands are separated by the band gap. Electrons can overcome the band gap energy by thermal excitation. The larger the band gap is compared to the thermal energy $\left(k_{B} T\right)$, the more electrical insulating the material. For example, diamond has a band gap of about $5.5 \mathrm{eV}$ at $300 \mathrm{~K}$ [14], that is over $200 k_{B} T$, which means electrons are barely excited into the conduction band [15] (Fig. 2.1, insulators). If the gap size is small enough so that electrons can be thermally excited into the conduction band, materials are classified as semiconductors (Fig. 2.1, intrinsic semiconductors). For instance, the band gap of intrinsic (i.e. without dopants) crystalline $\mathrm{Si}$ is $1.1 \mathrm{eV}$ at $300 \mathrm{~K}$ [16], which leads to a carrier density of $\sim 10^{10} \mathrm{~cm}^{-3}$ and finite conductivity $[13,15]$.

There is no sharp boundary between semiconductors and insulators, in terms of the gap size. In practice, intentionally introducing impurities, referred to as 'doping' is used to control the carrier concentration $[17,18]$. Doping of intrinsic semiconductors introduces extra charge carriers into the conduction band ( $n$-type doping) or the valence band ( $p$-type doping) (Fig. 2.1, n-type and p-type semiconductors), thereby increasing the conductivity [13]. The impurities are referred to as donors and acceptors, respectively.

When there is no band gap, the material is a metal. The missing band gap is due to either an overlap of the valence band and the conduction band or an originally partially filled valence band [13]. The lack of a band gap implies that there are already empty states for electrons to occupy within the same band. Therefore, metals are much more conducting than insulators and semiconductors.

\section{Charge carriers}

The charge carriers in a solid are not free electrons because of their interactions with their surroundings. These interactions are normally taken care of in band theory by defining quasiparticles with a renormalized mass $[19,20]$. As mentioned above, holes are valence band energy states which are not occupied by electrons. A hole is usually generated by thermally exciting an electron into the conduction band or by p-type doping [21, 22]. A hole has a positive charge and an opposite spin to the electron it replaces. Similar to electron quasiparticles, holes also have an effective mass. For both electrons and the holes, the effective mass can be derived from their respective dispersion relations. An electron (or a hole) combined with the deformation of its adjacent lattice structure can be treated as a quasiparticle referred to as a polaron [23]. The polaron concept is relevant in organic electronics, because organic materials are usually less rigid than inorganic materials [23, 24]. The polaron spin and charge are derived from the electron (or hole) constituting the polaron. Two nearby polarons with the same charge sharing a common deformation can form a 
bipolaron [25]. The bipolaron spin state (singlet or triplet) depends on the spins of the two polarons constituting the bipolaron. When two polarons with opposite charge bound to each other under influence of the Coulomb force, they form a polaron pair [26]. A polaron pair is usually an intermediate state between an exciton and two separated polarons. Similar to a bipolaron, the spin state of a polaron pair depends on the spins of the two polarons which form the polaron pair. Sometimes bipolarons are also regarded as a kind of polaron pairs [27]. Spectroscopic analysis of the quasiparticle energies can help identifying the relevant transport mechanism [28, 29].

\subsection{Introduction to organic electronics}

2.2.1 Molecular orbitals, energy bands/levels, band conduction and hopping conduction

Molecular orbitals are mathematical functions describing the wave-like behavior of electrons in a molecule. They are used to calculate the probability of finding an electron in any specific region, which is in particular relevant for the outer electrons. In the molecular orbital approximation, the behavior of one electron is described in the electric field generated by the surrounding nuclei and the average distribution of the other electrons. The Pauli principle demands that two electrons occupying the same molecular orbital have opposite spins. In the ground state, the topmost filled level is called the highest occupied molecular orbital (HOMO). The lowest empty level is called the lowest unoccupied molecular orbital (LUMO) [30].

In organic materials, electrical conductivity relies on the delocalization of the molecular orbitals [31]. This delocalization occurs in conjugated organic materials with alternating single and double bonds. In those materials, the carbon atoms (sometimes also nitrogen, oxygen and sulfur atoms) form $\sigma$ bonds (Fig. 2.2(e)). In addition, their $\mathrm{p}_{z}$ orbitals overlap with each other and form new orbitals called $\pi$ orbitals, bridging the $\sigma$ bond (Figs. 2.2(d) and $1.2(e))[31,32]$. The $\pi$ electrons delocalize across the adjacent aligned p-orbitals.

In carbon-based materials, usually $\mathrm{sp}, \mathrm{sp}^{2}$ and $\mathrm{sp}^{3}$ hybridizations occur. For $\mathrm{sp}^{3}$ hybridization, no extra $p$ orbital is left for the conjugation as, for example, in diamond. For $s p\left(s p^{2}\right)$ hybridization, there are two (one) unhybridized $\mathrm{p}$ orbitals which form $\pi$ orbitals as, for example, in ethene (ethyne). In an approximate quantum mechanical theory of conjugated molecules, only the molecular orbitals of the $\pi$ electrons are considered [30]. Those molecular orbitals are single-electron wave functions, although there are many $\pi$ electrons. 
Other electrons, such as the $\sigma$ electrons and other inner shell electrons, are much more tightly bound than the $\pi$ electrons. Their Coulomb interaction with $\pi$ electrons is averaged, and incorporated in a potential term, together with the Coulomb interaction between the $\pi$ electrons and the nuclei. Correlations between $\pi$ electrons are neglected in the approximate theory, but are necessary to incorporate in more accurate descriptions [30, 33].
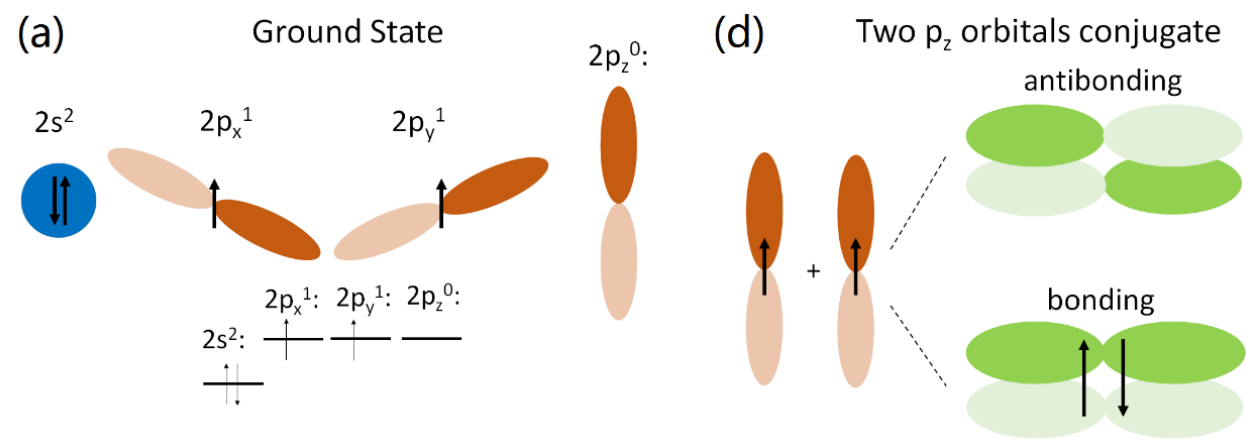

(b) Excited state

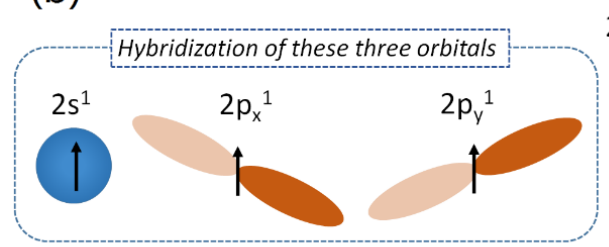
$2 p_{z}{ }^{1}$

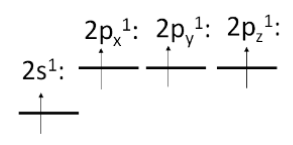

(c) After the $\mathrm{sp}^{2}$ hybridization
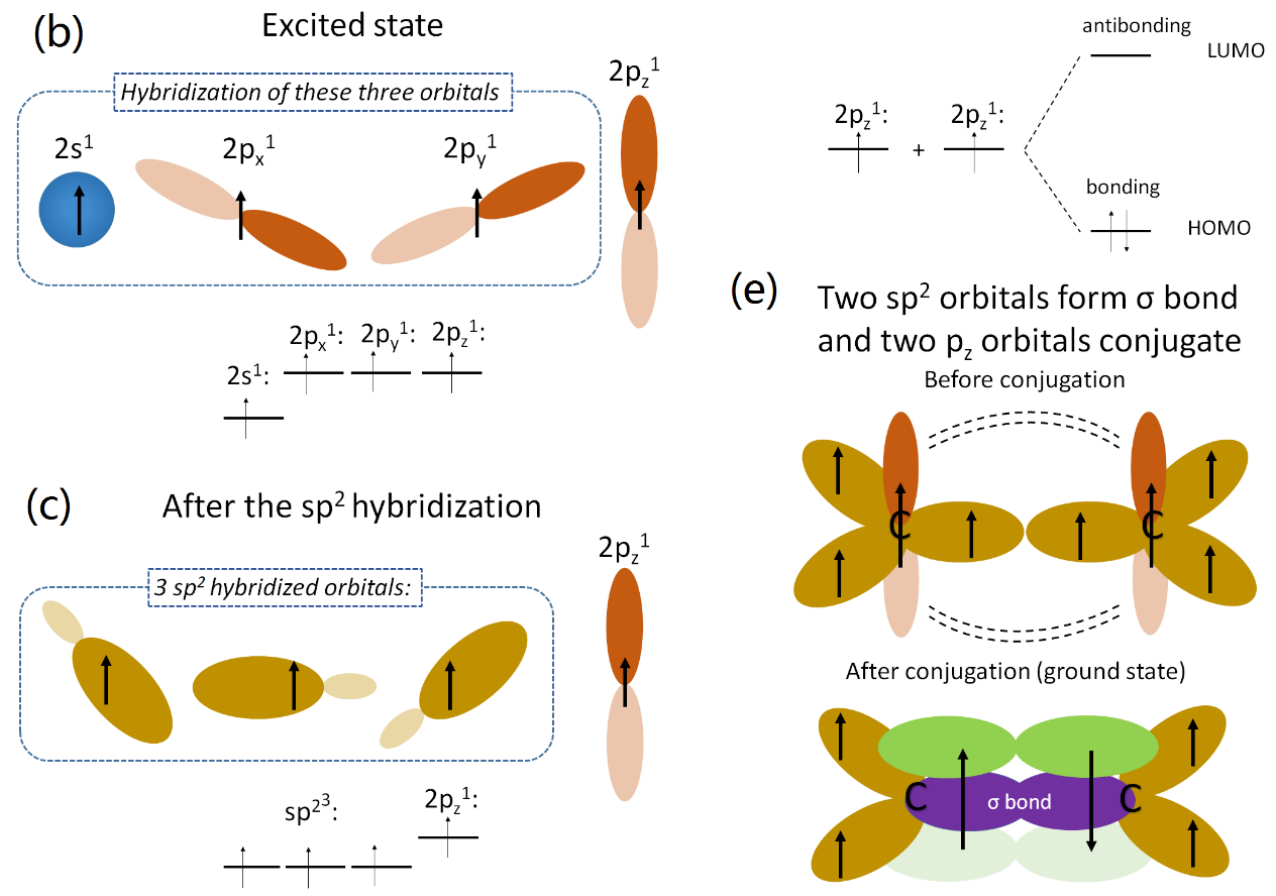

(e) Two $\mathrm{sp}^{2}$ orbitals form $\sigma$ bond and two $p_{z}$ orbitals conjugate Before conjugation

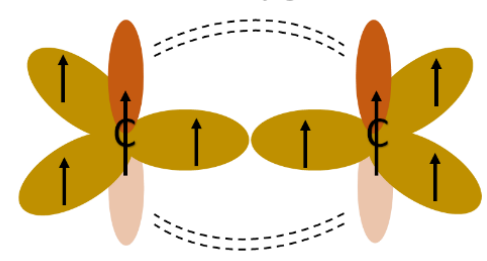

After conjugation (ground state)

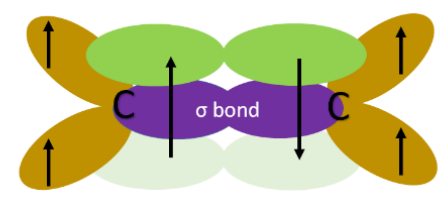

Figure 2.2: Schematic pictures of $\mathrm{sp}^{2}$ hybridization, conjugation between two carbon atoms (ethene), bonding and antibonding levels, HOMO and LUMO. Dark and light shading stand for positive and negative sign of the wave functions, respectively, similar to the Ref. [34]. Arrows stand for electrons (spins) occupying corresponding orbitals. (a) Ground state of a $\mathrm{C}$ atom, only show $2 \mathrm{~s}$ and $2 \mathrm{p}$ orbitals. (b) Excited state before hybridization. (c) $\mathrm{sp}^{2}$ hybridization. (d) Conjugation between two $\mathrm{p}_{z}$ orbitals. (e) Conjugation between two $\mathrm{C}$ atoms after $\mathrm{sp}^{2}$ hybridization. $\mathrm{C}-\mathrm{H}$ bonds are not shown here. 
Figure 2.2 shows the $\mathrm{sp}^{2}$ hybridization of two carbon atoms (an ethene system, the hydrogen atoms and the $\mathrm{C}-\mathrm{H}$ bonds are not shown) and the conjugation of the two carbon atoms, resulting in a bonding and antibonding orbital (Fig. 2.2(d)). The dark/light shading of all $p$ and $\pi$ orbitals indicate the positive/negative signs of the wave function, respectively. When the $p_{z}$ orbitals interact constructively without generating nodes in the final $\pi$ bonds, a bonding orbital is formed. In contrast, when a dark-shaded lobe and a light-shaded lobe interact destructively, leading to a node in the final $\pi$ orbital, an antibonding orbital is formed (Fig. 2.2(d)). The higher the molecular orbital energy, the more nodes appear in the wave function [32]. For the two-carbon system, the energy of the bonding orbital is lower than that of the antibonding orbital. In the ground state, only the bonding orbital is occupied by the two original $p_{z}$ electrons. The antibonding orbital is empty. So the bonding orbital is the HOMO of the two-carbon system and the antibonding one is the LUMO.

In a benzene ring, the delocalization occurs among 6 bonding carbon atoms. Figure 2.3 schematically shows the conjugation of $6 p_{z}$ orbitals, resulting in $6 \pi$ orbitals after conjugation. The orbitals $\pi_{1}, \pi_{2}$ and $\pi_{3}$ are bonding orbitals, $\pi_{4}, \pi_{5}$ and $\pi_{6}$ are antibonding orbitals. Their energy increases with the number of nodes in the wave function [30]. Hence, among the $6 \pi$ orbitals, two pairs of degenerate $\pi$ orbitals exist: $\pi_{2}$ and $\pi_{3}$, and $\pi_{4}$ and $\pi_{5}$. The $6 \mathrm{p}_{z}$ electrons occupy the bottom three levels in the ground state. So the energy level corresponding to the $\pi_{2}$ and $\pi_{3}$ orbitals is the HOMO. Similarly, the energy level corresponding to the $\pi_{4}$ and $\pi_{5}$ orbitals is the LUMO.

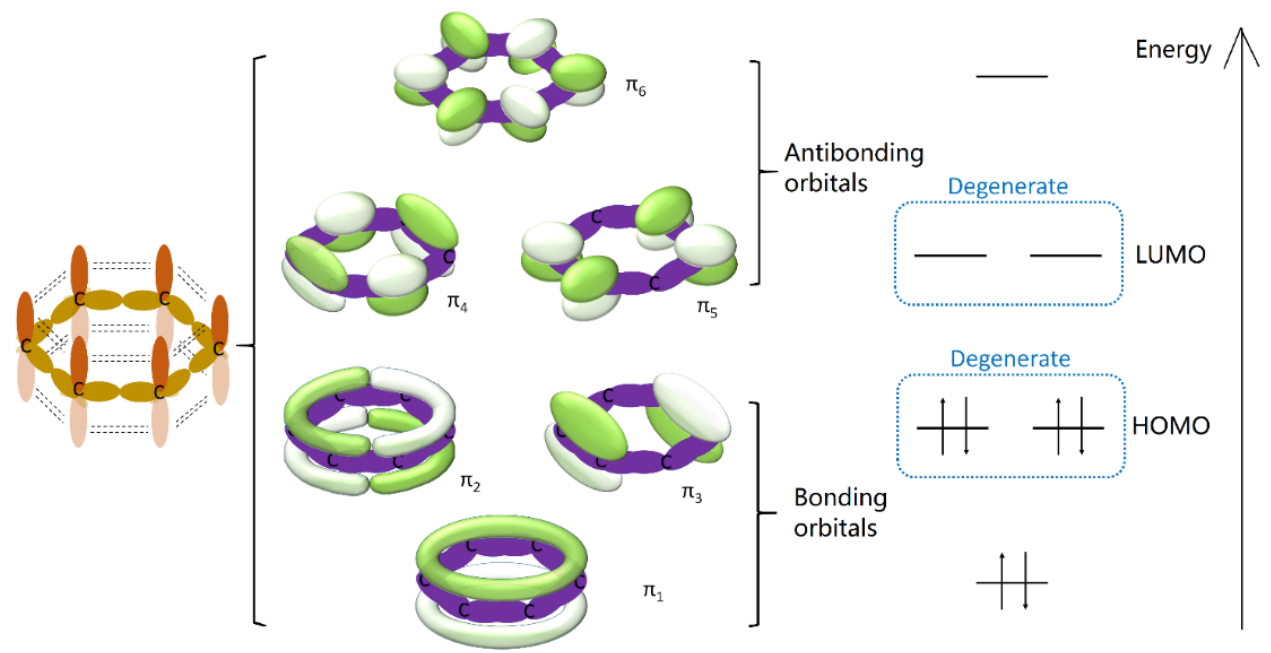

Figure 2.3: Schematic picture of benzene $\pi$ orbitals and the HOMO and LUMO energy levels. The C-H bonds are hidden. Dark and light shading correspond to the positive and negative sign of the wave function, respectively. 
In a solid, molecules will interact with their neighbors. If this interaction is strong enough, and the molecules are packed with long-range order, the molecular orbitals originally belonging to the individual molecules will be broadened into energy bands. The valence band results from the broadening of the HOMO levels, and the conduction band from the LUMO levels $[35,36]$, as shown in Fig. 2.4. The change of the ionization potential $\left(\Delta / I_{P}\right)$ and the electron affinity $\left(\Delta E_{A}\right)$ can be understood as the polarization energy of the surrounding molecules when an electron is removed from a molecule and when an electron is added to a molecule, respectively. In the solid state, adding an electron costs more energy, and ionizing an electron costs less energy, compared to the gas state [36].

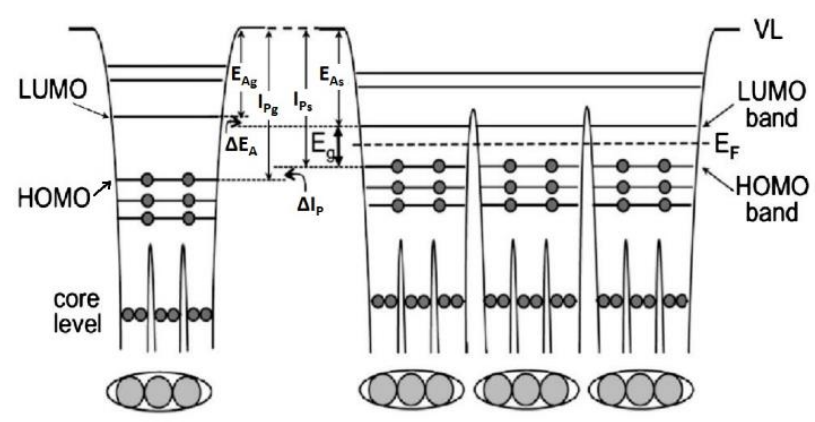

(a) Molecule (b) Organic solid (weak intermolecular interaction)

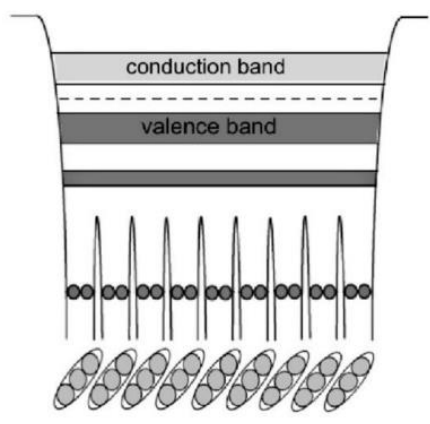

(c) Organic solid (stronger intermolecular interaction)

Figure 2.4. Evolution of electronic structure, from single molecules (a) to solids (b) and (c), reproduced from Ref. [36]. $E_{\mathrm{Ag}}$ and $E_{\mathrm{As}}$ are the electron affinity of the gas state (single molecules) and solid state respectively. $I_{\mathrm{Pg}}$ and $I_{\mathrm{Ps}}$ are the ionization potential of the gas state (single molecules) and solid state respectively. $\Delta /_{\mathrm{p}}$ and $\Delta E_{\mathrm{A}}$ are the change of the ionization potential and the electron affinity, respectively, when evolving from the gas state to the solid state. $E_{\mathrm{g}}$ is the energy (band) gap between LUMO (band) and HOMO (band). $E_{\mathrm{F}}$ is the Fermi energy and VL is the vacuum level. In (b), the LUMO and HOMO bands are very narrow so they are depicted as single lines [36].

However, due to lack of both long-range order and strong interaction between molecular orbitals, there is usually no band formed in disordered organic systems, such as organic polymers. When monomers polymerize into a polymeric chain, the HOMO and LUMO of the monomer units will disperse due to the interaction between monomers within the same chain or due to interaction between different chains [37, 38]. This dispersion gives rise to a certain density of states (DOS). When modeling these disordered materials, a specific DOS needs to be assumed, such as a Gaussian DOS [39] or an exponential DOS, as proposed by Miller and Abrahams [40].

In the band conduction regime, the charge carrier wave functions are delocalized over the whole material. With increasing temperature, lattice vibrations become more dominant, 
reducing the conductivity. So charge carrier mobilities usually decrease with increasing temperature for band-like conduction [41,42]. The temperature dependence can also involve many other factors, such as impurity trapping or impurity scattering [43-45]. In addition, the thermal energy can also inhibit band-like conduction in organic crystals [46].

Due to the weak inter-molecular Van der Waals interactions, the bands in molecular solids are usually very narrow (Fig. 2.4(b)). The $\pi$-orbitals are mainly delocalized within the individual molecules. Therefore, the band conduction model is often inappropriate for molecular materials $[35,36]$. In an alternative description, referred to as the hopping model, the wave functions are assumed to overlap only a little between neighboring molecules. Activated by phonons, charge carriers can hop from one molecular site to another [47]. Therefore, hopping is also called thermally assisted tunneling [48]. This mechanism is usually suppressed at lower temperature, although other activation mechanisms could enable hopping even at very low temperatures [48]. The hopping model is generally accepted to describe conduction in disordered organic systems. As mentioned above, even for ordered systems, there could be a crossover between the band conduction regime and the hopping conduction regime due to thermal bandwidth narrowing [42, 49] or due to electrical polarization in the nearby environment $[41,50]$.

The energy gap between the HOMO and LUMO levels of many bulk conjugated organic materials is around $2 \mathrm{eV}$. This gap size leads to semiconducting properties of those organic materials. Thus those materials are also called organic semiconductors (OSCs). In organic semiconductors, the alignment of the work function of the contact metal electrodes with the HOMO/LUMO levels is very important, because that energy difference largely affects the injection of charge carriers [51]. This effect will be briefly described in the discussion of two-terminal devices and organic field-effect transistors below.

\subsubsection{Why organic materials?}

There are several advantages of organic materials compared to inorganic materials. Organic materials can be processed by low-cost and low-temperature techniques, such as inkjet printing [52]. Organic electronics is very promising for future flexible electronic devices. In addition, organic materials are low-weight. Hence, future wearable electronic devices could be realized [53-55]. Organic electronic devices could be made biocompatible and of great use in medical diagnosis and monitoring [56].

Due to the low atomic number of the dominant elements in organic materials $(C, H, O, N)$, the spin-orbit coupling is generally much weaker than in inorganic materials, which is 
advantageous for spin-orbit-related spin relaxation [23]. A long spin-relaxation time offers the opportunity to detect and manipulate the spins of charge carriers during electrical transport, making organic materials promising for various spintronics applications. For example, in organic spin-valve devices [57], spin-polarized charge carriers are injected from one ferromagnetic electrode into organic materials, and transport to another ferromagnetic electrode. The resistance of the devices is depending on the alignment of the magnetization orientations of the two electrodes. Another application is spin-polarized light-emitting diodes [58]. In those devices, the two electrodes for the injection of electrons and holes are both ferromagnetic. Both the conductivity and electroluminescence of the devices can be controlled by an external magnetic field.

\subsubsection{Subfields of organic electronics}

Since roughly the mid of the last century, a lot of progresses has been made in the research field of organic electronics, ranging from materials design, synthesis, and characterizations to device design, fabrication and measurement techniques, and real-life applications. Below we discuss some basic device structures often applied in organic electronics.

The most basic layout of an organic electronics device is a two-terminal configuration. Having two metal electrodes, one can either apply a voltage or current, and measure the resulting current or voltage, respectively. Two-terminal devices can have a lateral (planar) or vertical geometry. In the lateral geometry, the two metal electrodes are in the same plane as the intermediate organic material. In the vertical geometry, one electrode is above the intermediate organic material and the other one below. The intermediate organic material can consist of a single molecule, a (self-assembled) molecular monolayer, a singlecrystal or bulk film consisting out of small molecules or polymers.

In a two-terminal organic semiconductor device, charge carriers are injected from one electrode into the organic material and transported to the other one. At the injecting electrode, a charge carrier needs to overcome an energy to be injected. This energy is referred to as the contact barrier (or energy barrier). The contact barrier types can be Ohmic or injection-limited, which can be determined by experimental transport characteristics [59]. Organic semiconductors usually have low conductivities. The injected charge carriers become space charge (excess charge) nearby the injecting electrode, building up electric potential and hence limiting the charge carrier injection. When the space charge is maximal, the obtained maximum current in an organic semiconductor is referred to as space charge limited current (SCLC) $[59,60]$. The SCLC is described by the Mott-Gurney equation [61]: 


$$
J_{\mathrm{SCLC}}=\frac{9}{8} \varepsilon \mu \frac{V^{2}}{L^{3}}
$$

where $\varepsilon$ is the dielectric constant of the organic semiconductor, $\mu$ is the charge carrier mobility, $V$ is the applied voltage and $L$ is the distance between the electrodes. From the equation we can see that SCLC has a power law dependence on the voltage. It has also been mentioned that the exponent can be larger than 2 [62]. The mobility can be electric-field dependent. It has also been reported that the mobility is also depending on the charge carrier density [63].

SCLC implies an Ohmic contact. In the SCLC regime, the charge transport in the bulk of organic material is dominant. In contrast, when device performance is determined by the contact barrier, that is, the current in the semiconductor is limited by the injection, the current is referred to as injection limited current (ILC), and the type of the contact barrier is injection-limited $[59,64]$.

It is often considered that charge transport in organic semiconductors includes two components, drift and diffusion current. As mentioned previously, in disordered organic systems, the HOMO and LUMO disperse with a certain density of states. Van Mensfoort et al. [65] have developed a drift-diffusion model. The model assumes a Gaussian density of states and takes account of the enhancement of the diffusion coefficient, the carrier density and the field-dependent mobility due to the Gaussian density of states.

In Chapter 5, we investigate a two-terminal vertical organic semiconductor device where an organic polymer layer is sandwiched between top and bottom metal contacts. We use the drift-diffusion model to explain the transport properties in these devices.

Organic field-effect transistors (OFETs) is being researched extensively. In Chapter 4 and Chapter 6, we also report investigations on OFETs. This topic is introduced in detail in section 2.3 below.

In organic photovoltaics (OPVs) or organic solar cells, light energy is transformed to electrical energy. The light excites electron-hole pairs in the organic semiconductor. These pairs are then dissociated into free negative and positive charge carriers (e.g., electrons and holes, or negative and positive polarons, respectively) which move towards the corresponding electrodes thus providing electrical energy to the outside circuit [66]. If, on the other hand, one electrically injects the opposite charge carriers into the organic material and let them combine, photons are emitted in the case of radiative recombination. This is how organic light-emitting diodes (OLEDs) work [67]. 
Organic spintronics is an emerging research field covering spin-dependent charge transport in organic materials, organic spin valves (OSV) [57] and organic magnetoresistance (OMAR) [68-70], pure spin flow in organic materials, e.g., by pumping spin through organic materials [71] or by non-locally measuring the Hanle effect in organic materials [72]. We refer to the following review papers for an elaborate discussion and overview of the existing literature [72-74].

\subsection{Organic field-effect transistors}

In the beginning of the $20^{\text {th }}$ century, our civilization entered the electronics age when vacuum triodes were launched and then applied extensively in radio communication and telephones not long after. In a vacuum triode, the third electrode, usually a metal grid, was placed between the anode and cathode to control the electron flow emitted from the cathode. In 1926, Julius Edgar Lilienfeld described a device in which the electrons flow in a conductive solid and can be controlled by an electric potential applied to a third electrode [75]. This is the basic design of the field-effect transistor (FET). Replacing the vacuum tube by a solid material reduces the fragility, increases the mobility of the charge carriers, decreases the consumed energy, and allows for device miniaturization. However, this idea was not realized until the invention of metal-oxide (silicon dioxide)-semiconductor (silicon) FETs at Bell Labs in 1959 [76, 77]. Nowadays, with the development of semiconductor fabrication techniques, electronic devices based on FETs have become much more energy efficient and portable than before, and dominate our lives. With the success of silicon FETs, considering the advantages of organic materials mentioned above, extensive research and development of OFETs are ongoing and pushing the field forward.

The conductivity of the organic semiconductor can be tuned by introducing extra charge carriers through applying an external electrical gate potential. This is the basic mechanism of organic field-effect transistors (OFETs). The external electrical potential is not necessarily applied through a gate electrode, but can also result from other sources, e.g., the electrochemical environment [78].

Transistors can be operated in the enhancement mode and the depletion mode, depending on whether the transistors are off or on respectively when the gate voltage is zero $[79,80]$. For instance, considering a p-channel transistor, when the threshold voltage is larger than zero, it means that the transistor is still on when the gate voltage is zero. This transistor is a depletion-mode transistor. The two modes affect eventual applications of transistors in circuits and performances of circuits. 


\section{Device structure}

OFET devices consist of source and drain electrodes, an organic material, a gate electrode, and gate dielectric. Usually, the organic material is deposited as thin film, hence OFETs are also referred to as organic thin-film transistors (OTFTs). OTFTs often have one of the following four different device structures shown in Fig. 2.5 [81]. When the substrate is conducting, e.g., a highly doped silicon substrate, the substrate can be used as the bottom gate, as shown in Figs. 2.5 (a) and (c). In (a) and (c) one could make very short channel devices with lithography techniques. In the configurations of (b) and (d), usually the source and drain electrodes are evaporated and patterned by a shadow mask. The reason for using a shadow mask to pattern the top electrodes is that the organic semiconductor can be affected by the lithography resists and developers used for standard lithography methods, and acetone or dimethyl sulfoxide (DMSO) used in lift-off procedures [82, 83]. In Chapter 5 and Chapter 6, we demonstrate that a method which is referred to as wedging transfer [84] can be used to deposit metal contacts on top of organic semiconductors. This method can also be used for lateral thin film transistors. The advantages are that the contact between the electrode and the organic semiconductor is well-defined, and that the distance between the electrodes can be reduced to very small values in the configurations of (b) and (d). In this method, the electrodes are first patterned by lithography techniques on $\mathrm{SiO}_{2}$, and then transferred onto the organic semiconductor.

(a)

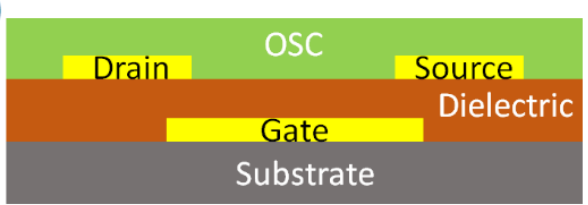

(c)

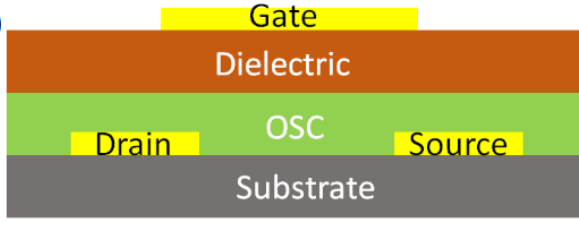

(b)

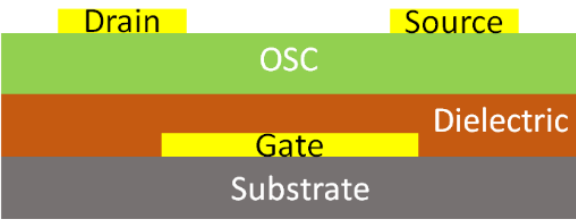

(d)

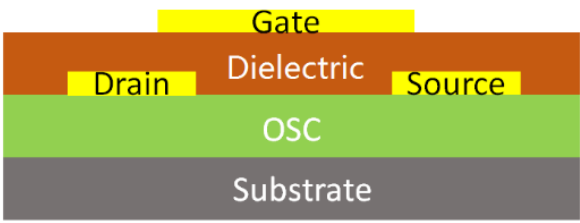

Figure 2.5: General OTFT device configurations. (a) Bottom gate bottom source-drain (SD) (coplanar bottom gate); (b) Bottom gate top SD (staggered bottom gate); (c) Top gate bottom SD (staggered top gate); (d) Top gate top SD (coplanar top gate). 


\section{Organic/metal interfaces: channel types and contact resistance}

Both p-type and n-type channel types are important for future organic electronic circuits. Combination of both types of OTFTs is necessary to build complementary circuits [85]. Complementary circuits can improve the noise properties [86], and increase the power efficiency because the transistors of one of the two carrier types is always off except during switching [87].

In inorganic devices, the channel type is usually determined by the dopant type. For example, in a $\mathrm{p}$-channel transistor, the source and drain are $\mathrm{p}+$-doped regions. Between the $\mathrm{p}$--doped regions is the $\mathrm{n}$-doped region where an inversion layer ( $\mathrm{p}$ channel) is formed when a sufficient gate voltage is applied. The transistor is now in the accumulation regime [88]. The inversion layer bridges the p+-type source and drain regions, and leads to the ON state of the transistor. If there is no inversion layer at all, the conductivity is very low because of reverse biased PN junctions [89]. The transistor is now in in the subthreshold regime [90]. However, in organic devices, charge carriers are usually injected from metal electrodes into the organic material. As mentioned above, the energy alignment between the work function of the metal contacts and the HOMO/LUMO levels of the organic material affects the eventual charge carrier injection [1,91]. Usually, low-work-function metals such as calcium are used to inject electrons into the LUMO, and high-work-function metals such as gold and platinum are used to inject holes into the HOMO.

The energy misalignment also introduces injection barriers at the metal/organic interfaces, giving rise to non-linear behavior in the current-voltage properties. If there is no barrier, the contacts exhibit ohmic behavior [92, 93].

Nevertheless, in real devices, the metal/organic interface could be very complicated. Diffusion of metal into the organic layer and transfer of electrons can introduce an interface dipole layer and gap states. Eventually, the alignment conditions are different from the expectations based on the metal work function and organic HOMO/LUMO levels [92, 94, 95]. It has also been reported that the charge carrier type can be changed by intentionally modifying the interfaces [96].

\section{Materials: polymers and molecules}

Organic semiconductors can be divided into polymers and small molecules. It is a challenging task to obtain long-range order in organic thin films. To obtain (poly)crystallinity, vacuum evaporation and heating the substrate can be used. One needs to be careful for the deformation of the metallic features under heating [97-100]. Post-annealing after the 
evaporation is also an option. Solution processes, spin coating or drop casting, are also often adopted for molecular materials as well. Solution-processable organic semiconductors could be used in future printed electronics [101]. However, solution processes usually are not as good as the evaporation processes concerning crystallinity. Sometimes small molecules are also not soluble enough for spin-coating. Adding alkyl chains to small molecules is a common way to increase solubility to improve solution processability, although this decreases the microscale order of films $[102,103]$. As for polymers, the spincoating process is usually used without too much trouble. It is also reported that hexamethyldisilazane (HMDS)/octadecyltrichlorosilane (OTS) pre-deposited on substrates could improve the crystallinity of organic materials [104].

\section{OFET transport properties}
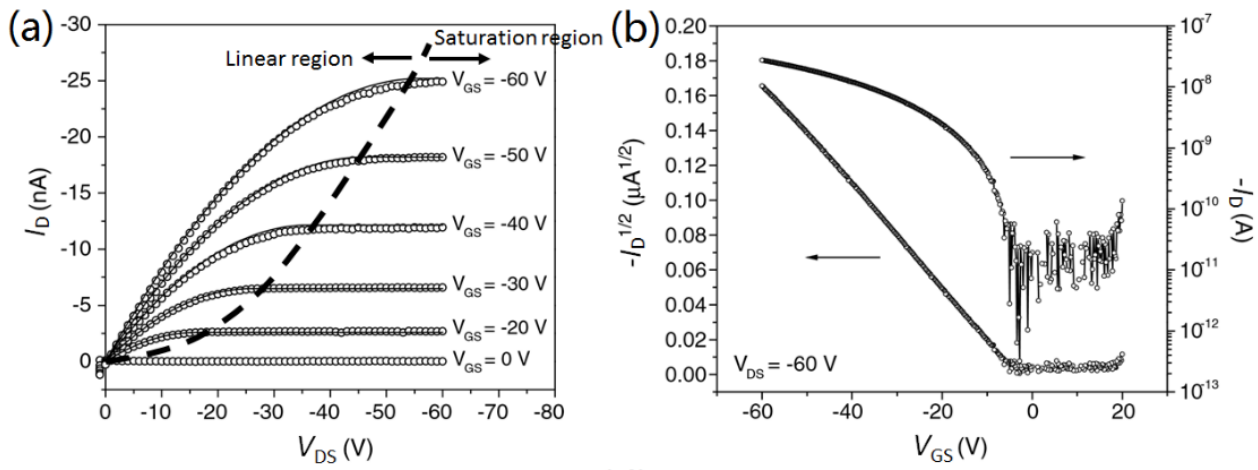

(c)

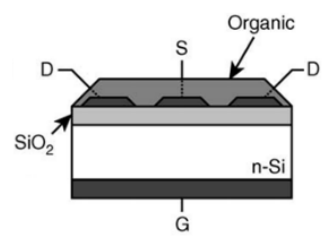

(d)

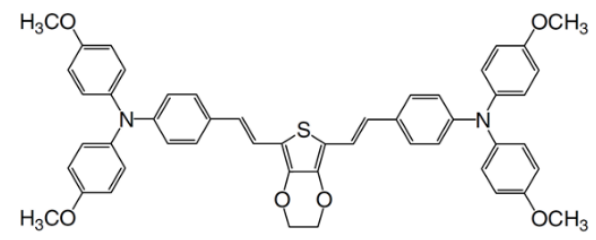

Figure 2.6: Typical output (a) and transfer (b) characteristics of p-type OFETs, reproduced from Ref. [62]. The thick dashed curve in (a) separates the linear and the saturation regime. (c) cross section of the OFET device (d) chemical structure of the organic semiconductor E, E-2,5-bis-[40-bis-(400-methoxyphenyl) amino-styryl]-3,4ethylenedioxy-thiophene. The channel length of the device is $50 \mu \mathrm{m}$, the $\mathrm{SiO}_{2}$ thickness is $200 \mathrm{~nm}$.

Typical OFET transport properties consist of output and transfer characteristics, of which examples are shown in Fig. 2.6. The output characteristic is a measurement of the drain current $I_{D}$ while sweeping the source-drain voltage $V_{S D}$ and keeping the source-gate voltage $V_{S G}$ constant. The transfer characteristic is a measurement of the drain current $I_{D}$ while 
sweeping the source-gate voltage $V_{S G}$ and keeping the source-drain voltage $V_{S D}$ constant. The output and transfer characteristics can be divided into two regimes, the linear regime and the saturation regime.

The output characteristics are described by the following two equations [105]:

linear regime: $\quad I_{\mathrm{D}}=\frac{W}{L} \mu_{\mathrm{lin}} C\left[-\frac{1}{2} V_{\mathrm{DS}}^{2}+\left(V_{\mathrm{GS}}-V_{\mathrm{th}}\right) V_{\mathrm{DS}}\right]$

saturation regime: $\quad I_{\mathrm{D}}=\frac{W}{2 L} \mu_{\mathrm{sat}} C\left(V_{\mathrm{GS}}-V_{\mathrm{th}}\right)^{2}$,

where $W$ is the channel width, $L$ is the channel length, $\mu_{\text {lin }}$ and $\mu_{\text {sat }}$ are the mobilities of the two regimes respectively, $C$ is the gate dielectric capacitance per unit area, $V_{\text {th }}$ is the threshold voltage (turn-off voltage) in the transfer curves.

In the linear regime, $V_{\mathrm{DS}}<V_{\mathrm{GS}}-V_{\mathrm{th}}$ [106], the channel is completely turned on by the source-gate voltage when $V_{\mathrm{GS}}>V_{\mathrm{th}}$, and not pinched off by the source-drain voltage. That is, the channel bridges the drain and source electrodes without being broken by a depletion region around the drain electrode. The conduction between the source and drain electrodes is mainly attributed to the channel conduction. Transistors working in the linear regime are like variable resistors. Because $I_{\mathrm{D}}$ depends linearly on $V_{\mathrm{GS}}$, this regime is also called the ohmic regime. Transistors in this regime can be used in circuits to realize resistive components whose resistance can be controlled by a feedback voltage. In the saturation regime $\left(V_{\mathrm{DS}}>V_{\mathrm{GS}}-V_{\mathrm{th}}\right)$, the channel is partially pinched off by the drain voltage in the vicinity of the drain electrode. In the pinched-off area, charge carriers are in the SCLC regime $[107,108]$. So in the saturation regime, the conduction consists of both SCLC and channel conduction. The saturation regime is also referred to as the active region. Transistors normally operate in the saturation regime for reasons of amplification, because the on/off ratio for the same source-gate voltage swing $\left(\left|V_{\mathrm{GS}}-V_{\mathrm{th}}\right|\right)$ is larger in the saturation regime, compared to that in the linear regime.

The mobility is also very important. The main reason is that the mobility is related to the operational frequency of OFETs. It is reported that the cutoff frequency of OFETs is proportional the transconductance $\left(\partial I_{\mathrm{D}} / \partial V_{\mathrm{GS}}\right)$ which is related to the mobility according to the Eqs. 2.2 and $2.3[109,110]$. It has also been mentioned that organic semiconductor with high mobilities $\left(1-10 \mathrm{~cm}^{2} \mathrm{~V}^{-1} \mathrm{~s}^{-1}\right)$ may meet the mobility requirements of the active-matrix which is used to control OLEDs [111]. Nowadays, the mobility of an OFET has already been improved to a high value which even exceeds that of the amorphous silicon devices (0.5-1 $\left.\mathrm{cm}^{2} \mathrm{~V}^{-1} \mathrm{~s}^{-1}\right)$. For example, a hole mobility of $40 \mathrm{~cm}^{2} \mathrm{~V}^{-1} \mathrm{~s}^{-1}$ for pentacene crystalline thin films [112] and a hole mobility of $43 \mathrm{~cm}^{2} V^{-1} \mathrm{~s}^{-1}$ for rubrene single crystals [113] have been reported. 
For polymeric organic semiconductors, a hole mobility of $8 \mathrm{~cm}^{2} \mathrm{~V}^{-1} \mathrm{~s}^{-1}$ in PTVD-10-based FETs has been reported [114].

For many applications of OFETs, like organic light-emitting transistors or display pixel drivers, it is crucial to achieve high frequencies ( $10 \mathrm{MHz}$ ) to improve the device performance [115, 116]. Besides using organic semiconductors with high mobility, reducing the channel length can also increase the cutoff frequency. Hence, in Chapter 5 and Chapter 6, we report a fabrication method to realize vertical organic devices with ultrashort channel length. However, this may give rise to another problem which is discussed in the following section.

\section{Short-channel effect}

When the channel length is decreased without changing the other device parameters, the OFET performance starts to deteriorate due to the so-called short-channel effect. In Chapter 4 and Chapter 6, we investigate OFETs with a short channel length ( $\leq 100 \mathrm{~nm}$ ). We also observe transport properties exhibiting the short-channel effect.

There are several reasons for going to short-channel OFET devices. The first reason is to scale down OFET integrated circuits. The second reason is to increase the cut-off frequency of devices [109, 110]. The third reason is that short-channel devices could cover single crystal domains thereby limiting the negative effects of domain boundaries [117-119]. For small channel length, the electric field between the source and drain electrodes at low voltage can still be very high. The devices can thus operate at low voltages, while maintaining sizeable current densities, which is beneficial for implementation in low-power electronic devices $[120,121]$.

The short-channel effect has two main characteristics. Firstly, in the saturation region, the drain current becomes source-drain-voltage dependent. When the channel length is decreased further, the drain current has a power-law dependence on the source-drain voltage and does not saturate at all $[62,122]$. Secondly, the threshold voltage shifts, and the source-drain channel is more difficult to turn off. As a result, enhancement-mode transistors can become depletion-mode transistors. The threshold voltage is also drain voltage dependent [62].

The increase of the longitudinal (source-drain) electric field is generally considered to be the main reason of the short-channel effect $[62,123]$. For shorter channel length, the depletion region around the drain electrode grows because of the stronger longitudinal electrical field and comparable to the transverse (source-gate) electrical field. Hence, the effective channel length (i.e. the accumulation layer length) decreases. 
As the depletion region expands, SCLC becomes more and more dominant. The SCLC has power law dependence on the voltage, as mentioned previously [124]. So the drain current shows an increase instead of instead of saturation (Figs. 2.7(a) and 2.7(b)). As the channel length decreases further, the depletion region will finally bridge the source-drain channel, resulting in a bulk space-charge-limited drain current Hence, when decreasing the channel length, the drain current will first show a linear dependence on $V_{S D}$ in the saturation region, referred to as channel-length modulation (CLM) [62], as shown in Fig. 2.7(a). Then the $I_{\mathrm{D}}-$ $V_{\mathrm{DS}}$ characteristic obtains a power-law dependence (Fig. 2.7(b)). As for the transfer characteristics, the drain current becomes less and less dependent on the gate voltage as the channel length decreases. Therefore, the device cannot be completely turned off due to the short-channel effect.
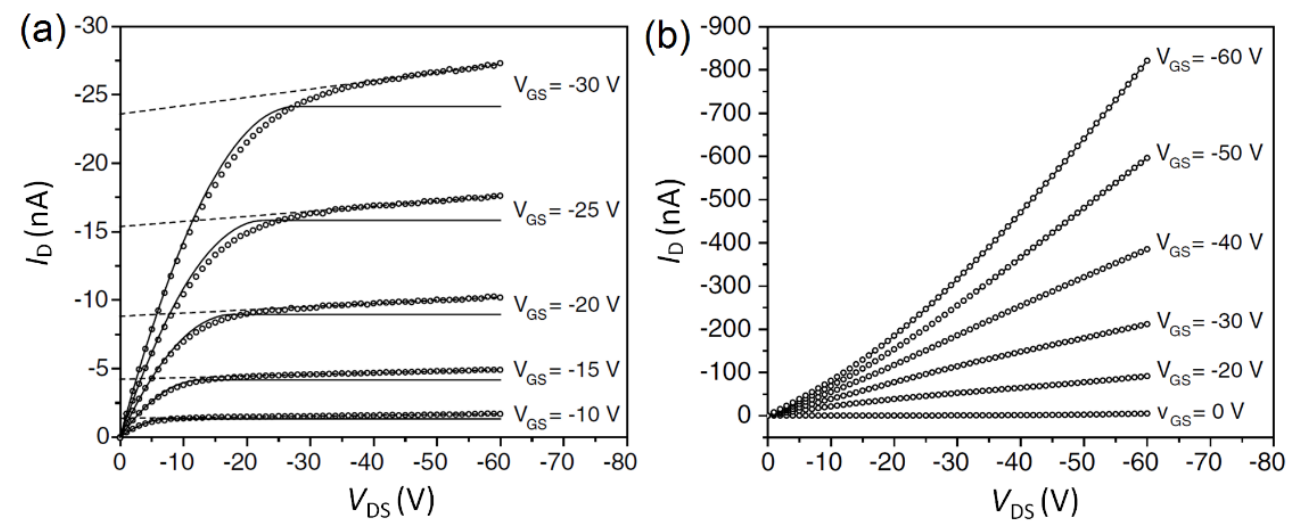

Figure 2.7: Output characteristics showing the short-channel effect. (a) and (b) show experimental (circles) output characteristics of three OFET devices, reproduced from Ref. [62]. Compared to the device of Fig. 2.6, the only difference with these two devices is the channel length: (a) $5 \mu \mathrm{m}$, (b) $1 \mu \mathrm{m}$. Solid lines in (a) are the fitting results using Eqs. (2.2) and (2.3). Dashed lines in (a) are linear fits to the data above pinch off $\left(V_{\mathrm{DS}}>V_{\mathrm{GS}}-V_{\mathrm{th}}\right)$.

To suppress the short-channel effect, one could increase the transverse field by increasing the gate capacitance, e.g., by using high-k dielectrics, decreasing the dielectric thickness, or increasing the gated area. Decreasing the organic thin-film thickness, either by simply coating a thinner organic film or using a special device structure, can shrink the bulk SCLC conduction region, thereby increasing the gate effect $[125,126]$. The above philosophy has already been very successfully applied in Si FETs. The FinFET structure (Fig. 2.8) is mainly for suppressing the short-channel effect by thinning the active Si region between source and drain, and increasing the gated area simultaneously. Thereby very short channel transistors even down to $10 \mathrm{~nm}$ can be realized $[127,128]$. Using the fabrication method reported in this thesis, we have realized ultrashort channel lengths down to $5 \mathrm{~nm}$ in vertical devices. 
Based on the above philosophy, apart from a surrounding gate which has also been fabricated in the vertical devices, reducing the lateral dimension of the devices is also expected to enhance the gate effect. This topic is discussed in Chapter 6.

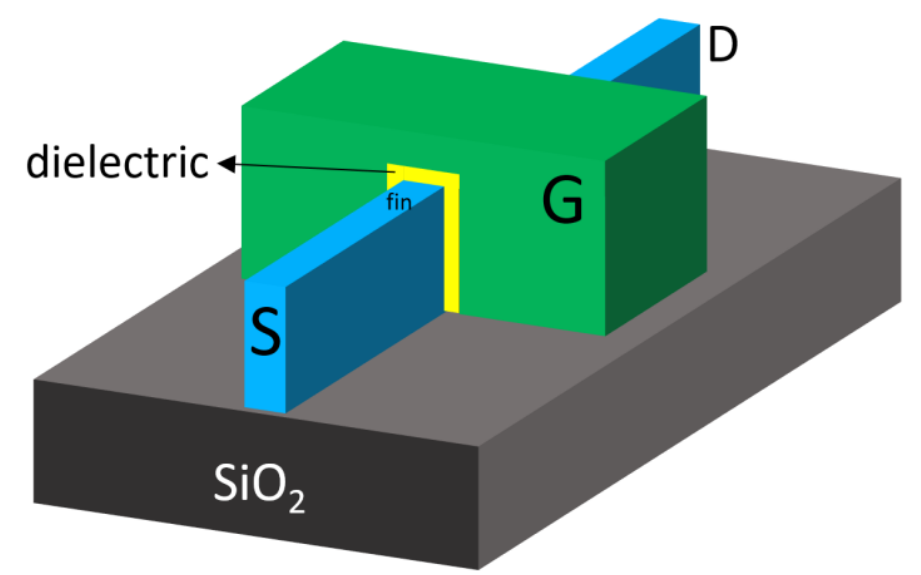

Figure 2.8: Schematic picture of a FinFET. The Si active region is limited in a vertical fin (blue). In addition, the fin is surrounded by a gate electrode (green) on three sides.

\section{Air-stability}

As reported in Chapter 4 and Chapter 5, we have observed that our devices were much more stable when measuring in vacuum $\left(<10^{-4} \mathrm{mbar}\right)$ than in the ambient atmosphere. In 1997, De Leeuw et al. reported that the ambient instability of the transport characteristics of organic semiconductors was mainly due to the reaction between charge carriers and $\mathrm{O}_{2} / \mathrm{H}_{2} \mathrm{O}$ in the ambient [129]. The instability can cause conduction degradation and it is also one of the main reasons of the bias stress effect, see below [130]. If the energy levels of the charge carriers are closer to the vacuum level, then it is easier for the reaction to happen. This is why the issue is more severe in most $n$-channel OSCs than in p-channel ones. Abundant work has been done to synthesize organic semiconductors with lower LUMO levels, that is, higher electron affinities. The usual way is to introduce electron-deficient substituents, such as perfluoralkyl $\left[-C_{n} F_{2 n+1}\right]$, cyanide $[-C N]$, fluoride $[-F]$, perfluorophenyl $\left[\mathrm{C}_{6} \mathrm{~F}_{5}-\right]$, carbonyl $[-\mathrm{C}(\mathrm{O})-]$, and imide $[-\mathrm{C}(\mathrm{O}) \mathrm{NHC}(\mathrm{O})-]$ groups to the molecules or monomers [102]. 


\section{Bias stress effect}

The bias stress effect usually presents itself as hysteresis in the transfer properties or as shift of the threshold voltage in a direction depending on the polarity of the applied gate voltage [130]. A similar phenomenon is also reported in Chapter 4 of this thesis. This effect decreases the stability of OFETs and the reliability of OFETs significantly. Many investigations have been done on this phenomenon and some underlying mechanisms have been proposed. Usually this effect is ascribed to trapping processes inside the organic semiconductor or gate dielectric, or at the gate dielectric/organic semiconductor interface. The traps could have several origins, such as air instability already mentioned above, structural-disorder-induced traps, or surface defects/contaminations in the gate dielectric [130-133].

The trapping inside the organic material could be associated with structural disorder of organic materials, impurities, and air instability. The structure-related trapping states commonly appear in disordered organic systems. Some reports mentioned that the mechanism behind it is local DOS broadening caused by local charge polarization [130, 134]. It has been reported that increasing the crystallinity could suppress this structure-related effect [135]. It was also found that light could also excite trapping states and cause the bias stress effect [136].

Residual contaminants on the dielectric surface is a well-known reasons for the bias stress effect $[130,137]$. Some researchers also suggested that hole carriers could transform to protons with the help of water in the measurement environment or at the OSC/dielectric interface, where the protons then diffuse into the dielectric layer in some p-channel OFETs [133]. To suppress the bias stress effect caused by the contaminants at the OSC/dielectric interface, self-assembled-monolayer (SAM) modification of the dielectric surface before deposition of OSCs is an efficient way which has already been widely adopted. HMDS or OTS treatment are most often used to decrease the bias stress effect. In addition, similar to the effect caused by local broadening of the DOS inside OSCs due to the structural disorder, the broadening could also happen close to the interface because of interaction between charge carriers in the accumulation layer and disordered dipoles in the gate dielectric. In this case, decreasing the interface structural disorder of the gate dielectric needs to be take into account $[50,130]$. 


\section{References}

1. Klauk, H., Organic electronics: materials, manufacturing, and applications. 2006: John Wiley \& Sons.

2. Klauk, H., Organic electronics II: more materials and applications. Vol. 2. 2012: John Wiley \& Sons.

3. Coropceanu, V., et al., Charge transport in organic semiconductors. Chemical reviews, 2007. 107(4): p. 926-952.

4. O'Neill, M. and S.M. Kelly, Ordered materials for organic electronics and photonics. Advanced Materials, 2011. 23(5): p. 566-584.

5. Clark, J. and G. Lanzani, Organic photonics for communications. Nature photonics, 2010. 4(7): p. 438-446.

6. Kahn, O., Molecular magnetism. VCH Publishers, Inc.(USA), 1993, 1993: p. 393.

7. Miller, J.S. and D. Gatteschi, Molecule-based magnets. Chemical Society Reviews, 2011. 40(6): p. 3065-3066.

8. Sugawara, T., H. Komatsu, and K. Suzuki, Interplay between magnetism and conductivity derived from spin-polarized donor radicals. Chemical Society Reviews, 2011. 40(6): p. 3105-3118.

9. Feynman, R.P., R.B. Leighton, and M. Sands, The Feynman lectures on physics, vol. 2: Mainly electromagnetism and matter. 1979: Addison-Wesley.

10. Jorgensen, W., The organic chemist's book of orbitals. 2012: Elsevier.

11. Ebbing, D. and S.D. Gammon, General chemistry. 2015: Nelson Education.

12. Liu, C.-y. and A.J. Bard, Pressure-induced insulator-conductor transition in a photoconducting organic liquid-crystal film. Nature, 2002. 418(6894): p. 162-164.

13. Streetman, B.G. and S. Banerjee, Solid state electronic devices. Vol. 4. 2000: Prentice Hall New Jersey.

14. Kittel, C., Introduction to solid state. 1966: John Wiley \& Sons.

15. Pan, L.S. and D.R. Kania, Diamond: electronic properties and applications. 2013: Springer Science \& Business Media.

16. Furukawa, S. and T. Miyasato, Quantum size effects on the optical band gap of microcrystalline Si: H. Physical Review B, 1988. 38(8): p. 5726.

17. Singleton, J., Band theory and electronic properties of solids. Vol. 2. 2001: Oxford University Press.

18. Erwin, S.C., et al., Doping semiconductor nanocrystals. Nature, 2005. 436(7047): p. 91-94.

19. Tse, W.-K., Many-body effects in graphene. 2008: ProQuest.

20. Tikhonov, E., Y.A. Uspenskii, and D. Khokhlov, Calculation of the spectrum of quasiparticle electron excitations in organic molecular semiconductors. Journal of Experimental and Theoretical Physics, 2015. 120(6): p. 1093-1100.

21. Thomas, G., Materials science: Invisible circuits. Nature, 1997. 389(6654): p. 907908.

22. Shklovskii, B.I. and A.L. Efros, Electronic properties of doped semiconductors. Vol. 45. 2013: Springer Science \& Business Media.

23. Naber, W., S. Faez, and W. Van Der Wiel, Organic spintronics. Journal of Physics D: Applied Physics, 2007. 40(12): p. R205. 
24. Pope, M. and C.E. Swenberg, Electronic processes in organic crystals and polymers. 1999: Oxford University Press on Demand.

25. Misra, P., Physics of condensed matter. 2011: Academic Press.

26. Ibrahim, M.I., Criteria for validating polaron pair dissociation in polymer-fullerene bulk heterojunction solar cells. Journal of Applied Physics, 2016. 119(15): p. 154504.

27. Janssen, P., et al., Tuning organic magnetoresistance in polymer-fullerene blends by controlling spin reaction pathways. Nature communications, 2013. 4.

28. Boehme, C. and J.M. Lupton, Challenges for organic spintronics. nature nanotechnology, 2013. 8(9): p. 612-615.

29. Bobbert, P., Is there more than meets the eye? Nature nanotechnology, 2013. 8(12): p. 887-887.

30. Salem, L., Molecular orbital theory of conjugated systems. 1966.

31. Cui, Z., Printed Electronics: Materials, Technologies and Applications. 2016: John Wiley \& Sons.

32. Anslyn, E.V. and D.A. Dougherty, Modern physical organic chemistry. 2006: University Science Books.

33. Mulliken, R.S., Spectroscopy, molecular orbitals, and chemical bonding. 1967: Laboratory of Molecular Structure and Spectra, University of Chicago.

34. Weiß, S., et al., Exploring three-dimensional orbital imaging with energydependent photoemission tomography. Nature communications, 2015. 6.

35. Silinsh, E.A., Organic molecular crystals: their electronic states. Vol. 16. 2012: Springer Science \& Business Media.

36. Holmes, R.J.D., Physics of organic semiconductors. 2012: Wiley-VCH.

37. Ajayaghosh, A., Donor-acceptor type low band gap polymers: polysquaraines and related systems. Chemical Society Reviews, 2003. 32(4): p. 181-191.

38. Kim, B.G., et al., Energy Level Modulation of HOMO, LUMO, and Band-Gap in Conjugated Polymers for Organic Photovoltaic Applications. Advanced Functional Materials, 2013. 23(4): p. 439-445.

39. Bässler, H., Charge transport in disordered organic photoconductors a Monte Carlo simulation study. physica status solidi (b), 1993. 175(1): p. 15-56.

40. Blakesley, J.C. and D. Neher, Relationship between energetic disorder and opencircuit voltage in bulk heterojunction organic solar cells. Physical Review B, 2011. 84(7): p. 075210.

41. Lezama, I.G. and A.F. Morpurgo, Progress in organic single-crystal field-effect transistors. MRS bulletin, 2013. 38(01): p. 51-56.

42. Hannewald, K. and P. Bobbert, Ab initio theory of charge-carrier conduction in ultrapure organic crystals. Applied physics letters, 2004. 85(9): p. 1535-1537.

43. Karl, N., Charge carrier transport in organic semiconductors. Synthetic Metals, 2003. 133: p. 649-657.

44. Bloch, A., et al., Low-temperature metallic behavior and resistance minimum in a new quasi one-dimensional organic conductor. Physical Review Letters, 1975. 34(25): p. 1561.

45. Jurchescu, O.D., J. Baas, and T. Palstra, The effect of impurities on the mobility of single crystal pentacene. arXiv preprint cond-mat/0404130, 2004. 
46. Troisi, A. and G. Orlandi, Charge-transport regime of crystalline organic semiconductors: Diffusion limited by thermal off-diagonal electronic disorder. Physical review letters, 2006. 96(8): p. 086601.

47. Kocherzhenko, A., Charge Transport in Disordered Molecular Systems. 2011: Aleksey Kocherzhenko.

48. Asadi, K., et al., Polaron hopping mediated by nuclear tunnelling in semiconducting polymers at high carrier density. Nature communications, 2013. 4: p. 1710.

49. Hannewald, K., et al., Theory of polaron bandwidth narrowing in organic molecular crystals. Physical Review B, 2004. 69(7): p. 075211.

50. Hulea, I., et al., Tunable Fröhlich polarons in organic single-crystal transistors. Nature Materials, 2006. 5(12): p. 982-986.

51. Hu, W., et al., Organic optoelectronics. 2012: John Wiley \& Sons.

52. Forrest, S.R., The path to ubiquitous and low-cost organic electronic appliances on plastic. Nature, 2004. 428(6986): p. 911-918.

53. Reuveny, A., et al., Ultra-flexible short-channel organic field-effect transistors. Applied Physics Express, 2015. 8(9): p. 091601.

54. Fukuda, K., et al., Fully-printed high-performance organic thin-film transistors and circuitry on one-micron-thick polymer films. Nature communications, 2014. 5.

55. Gundlach, D.J., Organic electronics: Low power, high impact. Nature Materials, 2007. 6(3): p. 173-174.

56. Irimia-Vladu, M., et al., Biocompatible and Biodegradable Materials for Organic Field-Effect Transistors. Advanced Functional Materials, 2010. 20(23): p. 40694076.

57. Xiong, Z., et al., Giant magnetoresistance in organic spin-valves. Nature, 2004. 427(6977): p. 821-824.

58. Nguyen, T.D., E. Ehrenfreund, and Z.V. Vardeny, Spin-polarized light-emitting diode based on an organic bipolar spin valve. Science, 2012. 337(6091): p. 204-209.

59. Woudenbergh, T.v., Charge injection into organic semiconductors. 2005, University of Groningen.

60. Simon, J. and J.-J. Andre, Molecular semiconductors: photoelectrical properties and solar cells. 2012: Springer Science \& Business Media.

61. Kiy, M., et al., Observation of the Mott-Gurney law in tris (8-hydroxyquinoline) aluminum films. Applied physics letters, 2002. 80(7): p. 1198-1200.

62. Haddock, J.N., et al., A comprehensive study of short channel effects in organic field-effect transistors. Organic electronics, 2006. 7(1): p. 45-54.

63. Vissenberg, M. and M. Matters, Theory of the field-effect mobility in amorphous organic transistors. Physical Review B, 1998. 57(20): p. 12964.

64. Varo, P.L., et al., Space-charge and injection limited current in organic diodes: $A$ unified model. Organic Electronics, 2014. 15(10): p. 2526-2535.

65. Van Mensfoort, S. and R. Coehoorn, Effect of Gaussian disorder on the voltage dependence of the current density in sandwich-type devices based on organic semiconductors. Physical Review B, 2008. 78(8): p. 085207.

66. Kaur, N., et al., Organic materials for photovoltaic applications: Review and mechanism. Synthetic Metals, 2014. 190: p. 20-26. 
67. Geffroy, B., P. Le Roy, and C. Prat, Organic light-emitting diode (OLED) technology: materials, devices and display technologies. Polymer International, 2006. 55(6): p. 572-582.

68. Reichert, T. and T.P. Saragi, Photoinduced magnetoresistance in organic field-effect transistors. Applied physics letters, 2011. 98(6): p. 063307.

69. Prigodin, V., et al., Anomalous room temperature magnetoresistance in organic semiconductors. Synthetic Metals, 2006. 156(9): p. 757-761.

70. Desai, P., et al., Magnetoresistance and efficiency measurements of Al q 3-based OLEDs. Physical Review B, 2007. 75(9): p. 094423.

71. Watanabe, S., et al., Polaron spin current transport in organic semiconductors. Nature Physics, 2014. 10(4): p. 308-313.

72. Yu, Z., Spin transport and the Hanle effect in organic spintronics. Nanoelectronics and Spintronics, 2015. 1(1).

73. Devkota, J., et al., Organic spin valves: a review. Advanced Functional Materials, 2016. 26(22): p. 3881-3898.

74. $\mathrm{Yu}, \mathrm{Z} .-\mathrm{G} .$, Suppression of the Hanle effect in organic spintronic devices. Physical review letters, 2013. 111(1): p. 016601.

75. Edgar, L.J., Method and apparatus for controlling electric currents. 1930, Google Patents.

76. Kahng, D., A historical perspective on the development of MOS transistors and related devices. IEEE Transactions on Electron Devices, 1976. 23(7): p. 655-657.

77. Dawon, K., Electric field controlled semiconductor device. 1963, Google Patents.

78. Capozzi, B., et al., Tunable charge transport in single-molecule junctions via electrolytic gating. Nano Lett, 2014. 14(3): p. 1400-4.

79. Lee, C.A., et al., Full-swing pentacene organic inverter with enhancement-mode driver and depletion-mode load. Solid-state electronics, 2006. 50(7): p. 1216-1218.

80. Lee, K.H., et al., Low-voltage pentacene transistor inverters using micro-contact printed nano-layer. Journal of Materials Chemistry, 2010. 20(4): p. 663-665.

81. Vidor, F.F., T. Meyers, and U. Hilleringmann, Flexible Electronics: Integration Processes for Organic and Inorganic Semiconductor-Based Thin-Film Transistors. Electronics, 2015. 4(3): p. 480-506.

82. Jia, H., et al., Patterning effects on poly (3-hexy/thiophene) organic thin film transistors using photolithographic processes. Organic electronics, 2007. 8(1): p. 44-50.

83. Gundlach, D., et al., Solvent-induced phase transition in thermally evaporated pentacene films. Applied Physics Letters, 1999. 74(22): p. 3302-3304.

84. Schneider, G.F., et al., Wedging transfer of nanostructures. Nano letters, 2010. 10(5): p. 1912-1916.

85. Laquindanum, J.G., et al., n-Channel organic transistor materials based on naphthalene frameworks. Journal of the American Chemical Society, 1996. 118(45): p. 11331-11332.

86. Bode, D., et al., Noise-margin analysis for organic thin-film complementary technology. IEEE Transactions on Electron Devices, 2010. 57(1): p. 201-208.

87. Klauk, H., et al., Ultralow-power organic complementary circuits. Nature, 2007. 445(7129): p. 745-748. 
88. Maddalena, F., Organic field-effect transistors for sensing applications. 2011: University Library Groningen][Host].

89. Spaepen, F., Solid State Physics: Advances in Research and Applications. Vol. 56. 2001: Academic Press.

90. Makovejev, S., et al., Assessment of global variability in UTBB MOSFETs in subthreshold regime. Journal of Low Power Electronics and Applications, 2014. 4(3): p. 201-213.

91. Meijer, E., et al., Solution-processed ambipolar organic field-effect transistors and inverters. Nature materials, 2003. 2(10): p. 678-682.

92. Ishii, H., et al., Energy level alignment and interfacial electronic structures at organic/metal and organic/organic interfaces. Advanced materials, 1999. 11(8): p. 605-625.

93. Kumatani, A., et al., On practical charge injection at the metal/organic semiconductor interface. Sci Rep, 2013. 3: p. 1026.

94. Scott, J.C., Metal-organic interface and charge injection in organic electronic devices. Journal of Vacuum Science \& Technology A, 2003. 21(3): p. 521-531.

95. Hill, I., et al., Molecular level alignment at organic semiconductor-metal interfaces. Applied Physics Letters, 1998. 73(5): p. 662-664.

96. Chua, L.-L., et al., General observation of $n$-type field-effect behaviour in organic semiconductors. Nature, 2005. 434(7030): p. 194-199.

97. Chen, X., et al., Ordered Au nanocrystals on a substrate formed by light-induced rapid annealing. Nanoscale, 2014. 6(3): p. 1756-1762.

98. Ge, X., et al., Tailoring the structure and property of Pt-decorated nanoporous gold by thermal annealing. The Journal of Physical Chemistry C, 2009. 113(17): p. 73797384.

99. Link, S., Z.L. Wang, and M.A. El-Sayed, How Does a Gold Nanorod Melt?\#. The Journal of Physical Chemistry B, 2000. 104(33): p. 7867-7870.

100. Siegel, J., et al., Annealing of gold nanostructures sputtered on polytetrafluoroethylene. Nanoscale research letters, 2011. 6(1): p. 1-6.

101. Yoo, B., et al., High-Performance Solution-Deposited n-Channel Organic Transistors and their Complementary Circuits. Advanced materials, 2007. 19(22): p. 4028-4032.

102. Usta, H., et al., Design, synthesis, and characterization of ladder-type molecules and polymers. Air-stable, solution-processable $n$-channel and ambipolar semiconductors for thin-film transistors via experiment and theory. Journal of the American Chemical Society, 2009. 131(15): p. 5586-5608.

103. Usta, H., A. Facchetti, and T.J. Marks, Air-stable, solution-processable n-channel and ambipolar semiconductors for thin-film transistors based on the indenofluorenebis (dicyanovinylene) core. Journal of the American Chemical Society, 2008. 130(27): p. 8580-8581.

104. Lim, S.C., et al., Surface-treatment effects on organic thin-film transistors. Synthetic Metals, 2005. 148(1): p. 75-79.

105. DiBenedetto, S.A., et al., Molecular Self-Assembled Monolayers and Multilayers for Organic and Unconventional Inorganic Thin-Film Transistor Applications. Advanced materials, 2009. 21(14-15): p. 1407-1433. 
106. Korodi, I.G., et al., Characterisation of organic field-effect transistors using metal phthalocyanines as active layers. physica status solidi (c), 2010. 7(2): p. 456-459.

107. Yan, D., H. Wang, and B. Du, Introduction to organic semiconductor heterojunctions. 2010: John Wiley \& Sons.

108. Oberhoff, D., et al., Arbitrary density of states in an organic thin-film field-effect transistor model and application to pentacene devices. IEEE Transactions on Electron Devices, 2007. 54(1): p. 17-25.

109. Klauk, H., U. Zschieschang, and M. Halik, Low-voltage organic thin-film transistors with large transconductance. Journal of Applied Physics, 2007. 102(7): p. 074514.

110. Kitamura, M. and Y. Arakawa, High current-gain cutoff frequencies above $10 \mathrm{MHz}$ in n-channel C60 and p-channel pentacene thin-film transistors. Japanese Journal of Applied Physics, 2011. 50(1S2): p. 01BC01.

111. Sirringhaus, H., 25th Anniversary Article: Organic Field-Effect Transistors: The Path Beyond Amorphous Silicon. Advanced materials, 2014. 26(9): p. 1319-1335.

112. Jurchescu, O.D., et al., Interface-Controlled, High-Mobility Organic Transistors. Advanced Materials, 2007. 19(5): p. 688-692.

113. Yamagishi, M., et al., High-mobility double-gate organic single-crystal transistors with organic crystal gate insulators. Applied physics letters, 2007. 90(18): p. 182117.

114. Chen, H., et al., Highly $\pi$-Extended Copolymers with Diketopyrrolopyrrole Moieties for High-Performance Field-Effect Transistors. Advanced Materials, 2012. 24(34): p. 4618-4622.

115. Wilbers, J.G.E., et al., Charge transport in nanoscale vertical organic semiconductor pillar devices. Scientific Reports, 2017. 7: p. 41171.

116. Kang, H., et al., Megahertz-class printed high mobility organic thin-film transistors and inverters on plastic using attoliter-scale high-speed gravure-printed sub-5 $\mu \mathrm{m}$ gate electrodes. Organic Electronics, 2014. 15(12): p. 3639-3647.

117. Chwang, A.B. and C.D. Frisbie, Temperature and gate voltage dependent transport across a single organic semiconductor grain boundary. Journal of Applied Physics, 2001. 90(3): p. 1342-1349.

118. Horowitz, G., Organic thin film transistors: From theory to real devices. Journal of materials research, 2004. 19(07): p. 1946-1962.

119. Granstrom, E.L. and C.D. Frisbie, Field effect conductance measurements on thin crystals of sexithiophene. The Journal of Physical Chemistry B, 1999. 103(42): p. 8842-8849.

120. Sawabe, K., et al., Current-Confinement Structure and Extremely High Current Density in Organic Light-Emitting Transistors. Advanced Materials, 2012. 24(46): p. 6141-6146.

121. Fischer, A., et al., An all C 60 vertical transistor for high frequency and high current density applications. Applied Physics Letters, 2012. 101(21): p. 213303.

122. $\mathrm{Xu}, \mathrm{Y}$. and P.R. Berger, High electric-field effects on short-channel polythiophene polymer field-effect transistors. Journal of applied physics, 2004. 95(3): p. 14971501.

123. Herlogsson, L., et al., Downscaling of organic field-effect transistors with a polyelectrolyte gate insulator. Advanced Materials, 2008. 20(24): p. 4708-4713. 
124. Austin, M.D. and S.Y. Chou, Fabrication of $70 \mathrm{~nm}$ channel length polymer organic thin-film transistors using nanoimprint lithography. Applied Physics Letters, 2002. 81(23): p. 4431-4433.

125. Tukagoshi, K., et al., Suppression of short channel effect in organic thin film transistors. Applied Physics Letters, 2007. 91(11): p. 113508.

126. Wang, J., Z. Zheng, and H. Sirringhaus, Suppression of short-channel effects in organic thin-film transistors. Applied physics letters, 2006. 89(8): p. 083513.

127. Hisamoto, D., et al., FinFET-a self-aligned double-gate MOSFET scalable to $20 \mathrm{~nm}$. IEEE Transactions on Electron Devices, 2000. 47(12): p. 2320-2325.

128. Huang, X., et al. Sub 50-nm finfet: Pmos. in Electron Devices Meeting, 1999. IEDM'99. Technical Digest. International. 1999. IEEE.

129. De Leeuw, D., et al., Stability of n-type doped conducting polymers and consequences for polymeric microelectronic devices. Synthetic Metals, 1997. 87(1): p. 53-59.

130. Sirringhaus, H., Reliability of organic field-effect transistors. Advanced Materials, 2009. 21(38-39): p. 3859-3873.

131. Zschieschang, U., et al., Bias stress effect in low-voltage organic thin-film transistors. Applied Physics A, 2009. 95(1): p. 139-145.

132. Egginger, M., et al., Current versus gate voltage hysteresis in organic field effect transistors. Monatshefte für Chemie-Chemical Monthly, 2009. 140(7): p. 735-750.

133. Sharma, A., et al., Proton migration mechanism for the instability of organic fieldeffect transistors. Applied Physics Letters, 2009. 95(25): p. 253305.

134. Lee, B., et al., Origin of the bias stress instability in single-crystal organic field-effect transistors. Physical Review B, 2010. 82(8): p. 085302.

135. Sekitani, T., et al., Suppression of DC bias stress-induced degradation of organic field-effect transistors using postannealing effects. Applied Physics Letters, 2005. 87(7): p. 073505.

136. Gu, G., M.G. Kane, and S.-C. Mau, Reversible memory effects and acceptor states in pentacene-based organic thin-film transistors. Journal of applied physics, 2007. 101(1): p. 014504.

137. Goldmann, C., D. Gundlach, and B. Batlogg, Evidence of Water-related Discrete Trap State Formation in Pentacene Single Crystal Field-Effect Transistors. arXiv preprint cond-mat/0508607, 2005. 


\section{Chapter 3}

\section{Nanoindentation with accurate positioning}

\subsection{Introduction and motivation}

In this chapter we introduce a fabrication method based on nanoindentation using atomic force microscope (AFM) for electron transport measurements on one-dimensional DXPloaded zeolite $\mathrm{L}$ crystals and two-dimensional electron systems in $\mathrm{LaAlO}_{3} / \mathrm{SrTiO}_{3}$ heterostructures. We investigate different nanoindentation methods and demonstrate a high-precision nanoindentation technique which is suitable for the fabrication of nanocontacts on the submicron DXP-loaded zeolites and $\mathrm{LaAlO}_{3} / \mathrm{SrTiO}_{3}$ samples.

Zeolite $L$ is an insulating aluminosilicate crystal with well-defined channels and cavities [1]. Figure 3.1 shows the zeolite $L$ framework and the dimensions of its main channels. Figure 3.1(a) shows the top view of a zeolite with $c$-axis perpendicular to the paper plane and the side view of one channel. The channels have $0.71 \mathrm{~nm}$ inner radius shown in Fig. 3.1(b). The channels can accommodate organic species. Our zeolites had already been filled with $\mathrm{N}, \mathrm{N}^{\prime}$ bis(2,6-dimethylphenyl)-perylene-3,4,9,10-tetracarboxylic diimide (DXP) molecules, shown in Fig. 3.1(b). The DXP molecules were loaded into the channels along the $c$-axis at high temperatures [2]. With close spacing between two neighboring molecules, the molecules form one-dimensional molecular wires. The filling rate was derived by thermogravimetric analysis of the concentration of inserted molecules $[3,4]$.

DXP are organic dye molecules. The DXP-loaded zeolite system had already been researched in terms of energy transfer through the DXP molecules [5]. DXP also has conjugated orbitals which could enable charge transport. The HOMO and LUMO of DXP are -3.9 and $-6 \mathrm{eV}$ respectively [6]. Therefore, the DXP-loaded zeolite is expected to have unique electrical transport properties due to the strictly one-dimensional constrain.

To perform electrical transport measurements, firstly the zeolites were distributed into monolayers on glass substrates [7] and then transferred to PEDOT: PSS/ITO substrates by the PDMS stamp transfer technique [8]. The PEDOT: PSS played as a conductive adhesion layer. Considering the air stability issue of the organic material and small diameters ( 150 $300 \mathrm{~nm}$ ) of the zeolites, previously a high-vacuum conductive-probe atomic force microscope (CP-AFM) was used to measure those DXP-loaded zeolites. We planned to embed those zeolites into devices to measure more transport properties with other equipments, such as AC transport measurements and temperature dependence 
measurements, and so on. And more intriguingly, if we could eventually fabricate devices with interesting electrical transport properties, for example, magnetic field dependent transport, then we could further put the DXP-loaded crystals into practical use such as magnetic field sensors based on the devices. Therefore, we designed a fabrication process using nanoindentation to embed the zeolites into devices. The fabrication process will be described below. Figure 3.2(b) shows a 3D schematic diagram of an envisioned zeolite device. SEM image of three zeolites are shown in Fig. 3.2(a).
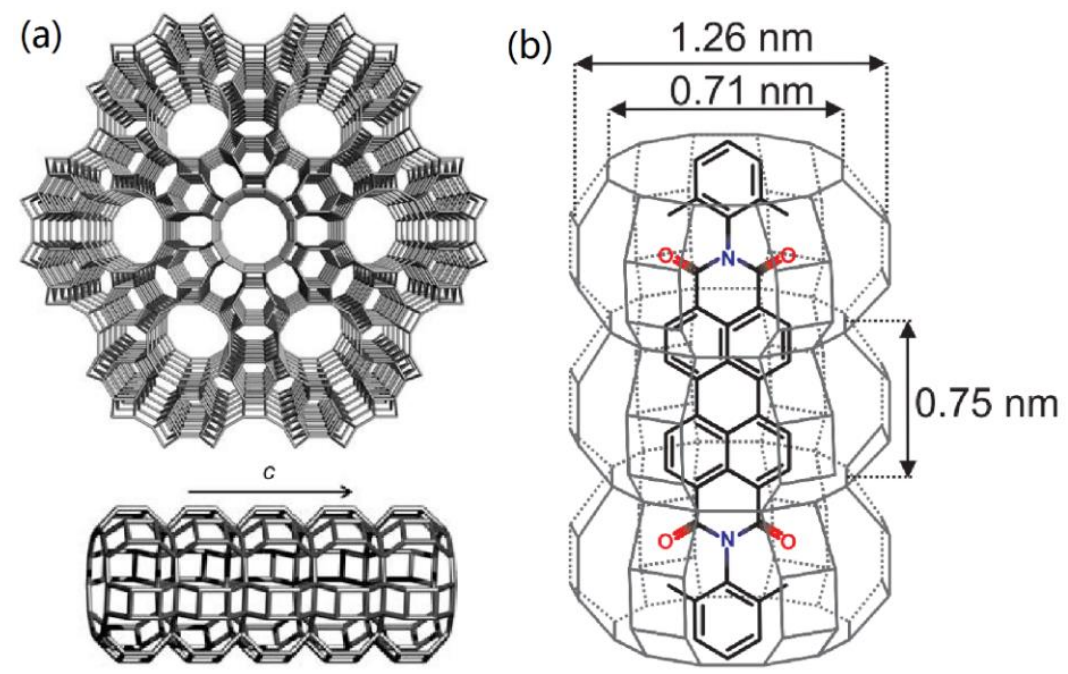

Figure 3.1: Zeolite framework and DXP molecule inserted in the channel of zeolite. (a) Top view (top, $c$-axis is perpendicular to the paper plane) and side view (bottom) of zeolite framework, reproduced from Ref. [1]. (b) A DXP molecule inserted in the channel and the dimensions of the channel.

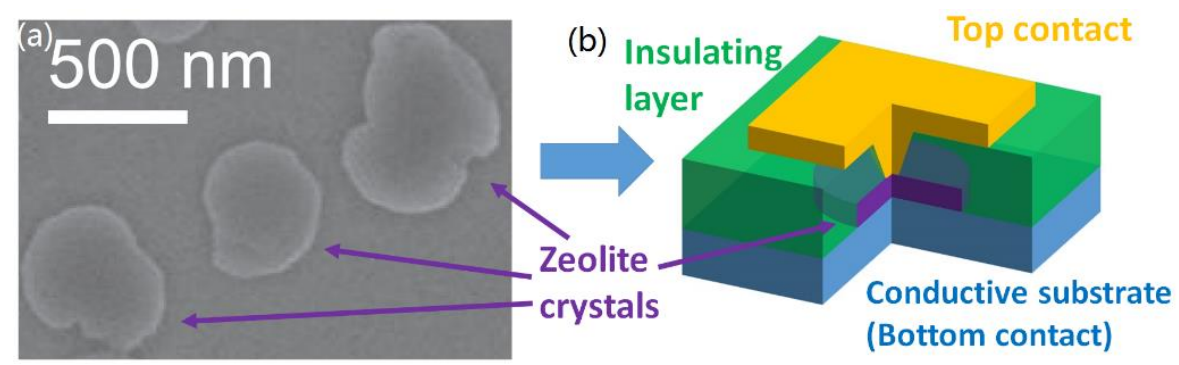

Figure 3.2: (a) SEM image of top view of three zeolites. (b) 3D schematic picture showing expected device structures containing a DXP-loaded zeolite.

Besides the zeolite device, we also suggest to fabricate metallic nanocontacts on $\mathrm{LaAlO}_{3} / \mathrm{SrTiO}_{3}$ heterostructures using the fabrication method for electron transport 
measurements on two-dimensional electron systems in the heterostructures. These experiments are presently carried out, but fall outside the scope of this thesis.

It has been reported that there are high-mobility 2-dimensional electron gases (2DEGs) at the interface between the two insulating oxide layers, $\mathrm{LaAlO}_{3}$ (LAO) and $\mathrm{SrTiO}_{3}$ (STO) [9-11]. This 2DEG layer has some interesting properties, such as superconductivity [12] and tunable superconductivity of tunneling transport from the 2DEG layer to the LAO surface through the LAO layer [13], different density of states (DOS) from 2DEGs in conventional semiconductors [10], strong spin-orbit coupling [14], signatures of magnetic scattering properties in the interface electrical transport and ferromagnetism at the interface $[15,16]$, and sensitivity to the electric potential at the LAO surface $[17,18]$. Owing to the small tipradius of atomic force microscope (AFM) probes (Fig. 3.3(a)), we are able to measure the tunneling spectroscopy in small regions by fabricating nanocontacts on the LAO/STO samples using the nanoindentation technique. Figure 3.3(b) is a schematic picture showing the expected device structure fabricated with a similar process of the zeolite-crystal device and the tunneling measurement setup. With small junction areas, suppression of structural fluctuations is expected to be suppressed. In addition, biasing the top Au contacts can also locally tune the electron density of the interface, which thereby affects the in-plane electrical transport at the interface, as reported in Refs. [17, 18]. Resistance switching behavior of vertical tunneling transport was also reported. The phenomenon is likely due to the ionic movement of charged oxygen vacancies in the LAO layer under high electric fields ( MV/cm) because of the ultrathin LAO layer (only a few unit cells) [19]. These devices are suitable for realization of the switching behavior as well.
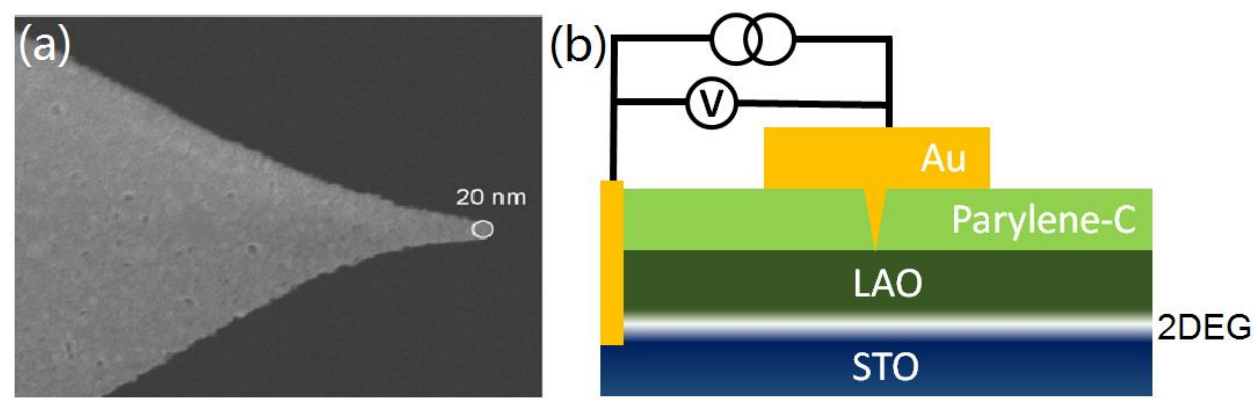

Figure 3.3: (a) SEM image of the tip a PtSi non-contact-mode AFM probe. Reproduced from NANOSENSORS product brochure. (b) Schematic diagram of desired device structure and the simplified tunneling measurement setup. 


\subsection{Nanoindentation}

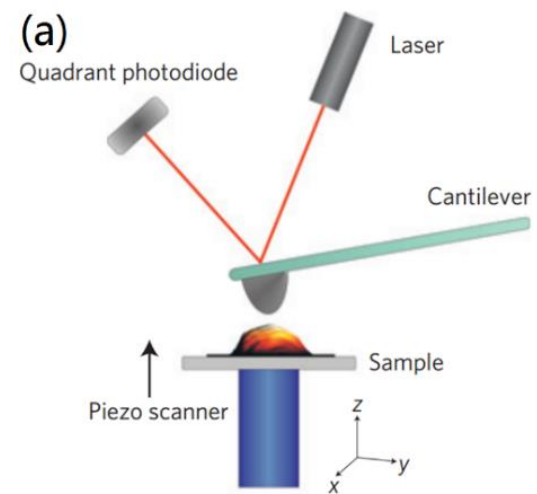

(b)

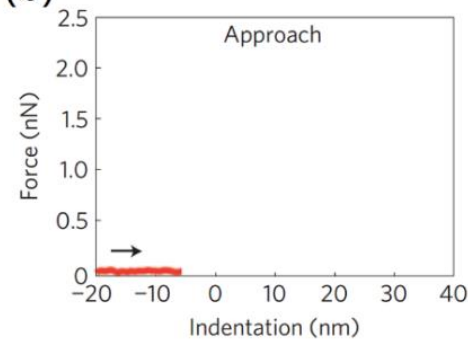

(c)

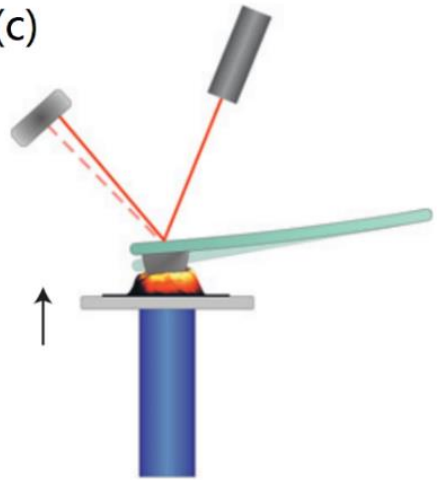

(d)

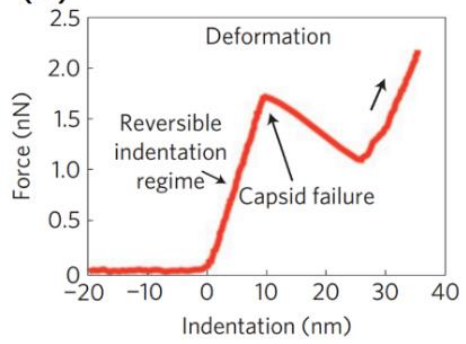

Figure 3.4: Schematic representation of nanoindentation experiment on a virus, reproduced from Ref. [20]. (a) Piezo is extending. (b) The AFM probe has not yet touched the sample, so there is no force. (c) The AFM probe has touched the sample, so the laser on the photodetector is deflected and this signal is transformed to force and plotted in (d) as a function of indentation.

The indentation technique has been used to investigate the mechanical properties of materials, such as hardness, Young's modulus, the yield stress, the elastic and plastic properties, etc. [21-24]. Nanoindentation is a variation on the indentation technique, used to measure the mechanical properties at the nanoscale. Nanoindentation is usually performed with an atomic force microscope (AFM), where the AFM probes are used as indenters. Because of the small tip-radius of the AFM probes, the mechanical information has nanoscale resolution. Nanoindentation with an AFM probe is schematically shown in Fig. 3.4. During the nanoindentation process, the sample stage is actuated by the $z$-piezo controller and moved towards the AFM probe. Alternatively, the AFM probe can be actuated by the $z$-piezo controller, and moved towards the sample. A laser is cast on the back surface of the cantilever of the AFM probe, and then reflected to a four-quadrant photo detector. Hence, bending of the cantilever due to the interaction between the tip of the AFM probe and the sample is recorded in the form of the deflection of the laser spot in the four-quadrant photodetector. This laser signal can be transformed to a force signal, 
which is usually related to the hardness, Young's modulus, elastic and plastic properties, etc., of the sample. In this way one can derive the mechanical properties of the sample from the relationship between the force and the z-piezo extension, which is usually referred to as the force-distance curve or force-z curve.

Sample to be measured

Insulating layer

Junction material

Contact electrodes

(normal metal, FM material, etc.)

1)

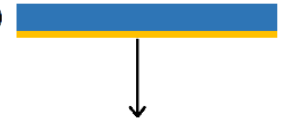

(2)
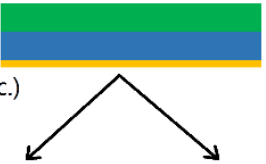

(3)

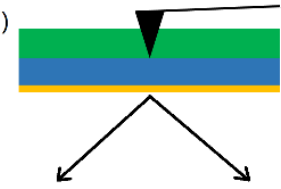

(5)

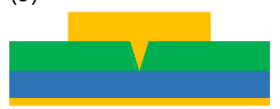

(6)

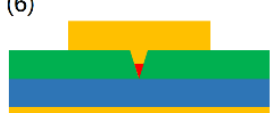

(4)

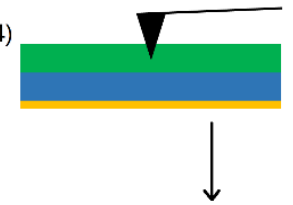

(7)

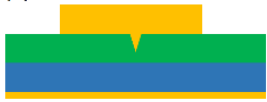

(8)

(9)

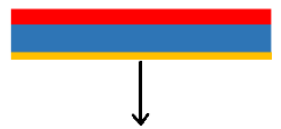

(10)

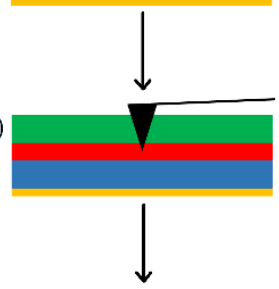

(11)

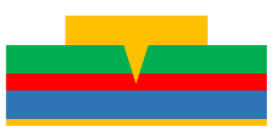

Figure 3.5: Schematic diagram of fabrication processes using the nanoindentation. Each number indicates a fabrication step. (1) Sample to be measured deposited on bottom electrodes. (2) Cover soft insulating layer for nanoindentation. Nanoindentation through the insulating layer (3), or leave some insulating material (4). Deposit top contacts $(5,7)$ or fill the hole with junction material before covering top contacts $(6)$. (8) Cover junction material before deposition of insulating layer. (9) Cover insulating layer. (10) Nanoindentation without indenting through the insulating layer. (11) Deposit top contacts.

Recently, nanoindentation has been adopted to fabricate nanodevices [25-29], schematically shown in Fig. 3.5. First, the simplest way is that we can employ nanoindentation to fabricate nanocontacts on samples to be measured, as shown in the process $(1) \rightarrow(2) \rightarrow(3) \rightarrow(5)$ of Fig. 3.5. Or we can fill in nanoindented holes with junction materials before covering top contacts $((1) \rightarrow(2) \rightarrow(3) \rightarrow(6))$. Nanoindentation can also be executed using conductive-tip AFM (CT-AFM), also known as conductive-probe AFM (CPAFM), especially when the underlying substance beneath the insulating layer is conductive. The conductivity between the tip and the underlying conductive part can be monitored realtime during the nanoindentation. In this way we can also leave a very thin insulating layer as a tunneling layer, instead of completely indenting through the insulating layer, shown in the process (1) $\rightarrow(2) \rightarrow(4) \rightarrow(7)$ of Fig. 3.5 .

There can be many varieties of the nanoindentation fabrication method. For example, we could firstly deposit junction material and then execute other steps, as shown in the process $(8) \rightarrow(9) \rightarrow(10) \rightarrow(11)$, eventually ending up with a similar junction device to (6) of Fig 3.5. 
From the above introduction we can see that nanoindentation could replace sophisticated lithography processes in nanodevice fabrication to make metallic contacts or nanojunctions. Based on the above introduction, we designed a device fabrication method using the nanoindentation to realize the envisioned devices shown in Figs. 3.2 and 3.3. The fabrication method is described below.

\subsection{Device fabrication}

(a)

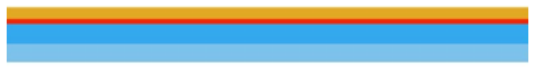

(b)

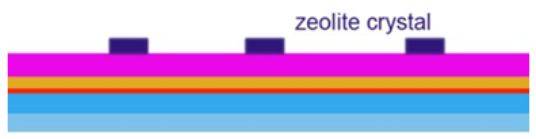

(c)

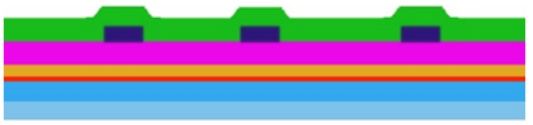

(d)

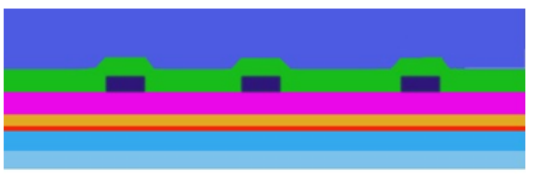

(e)

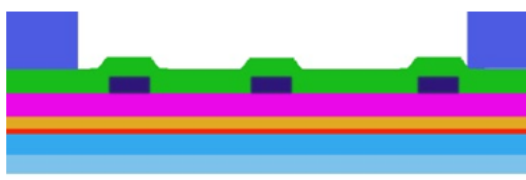

(f)

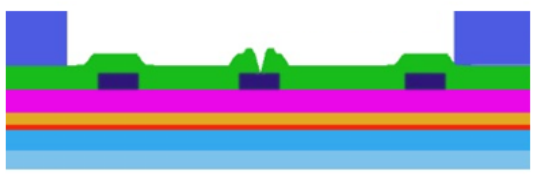

(g)

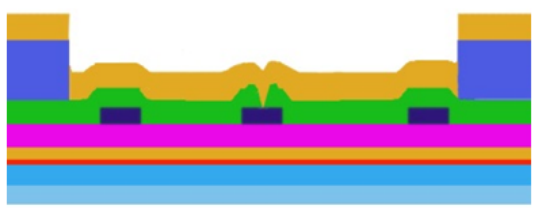

(h)

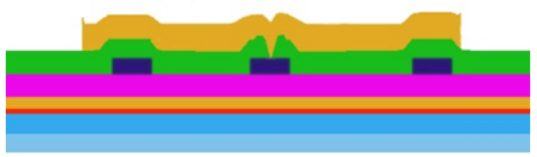

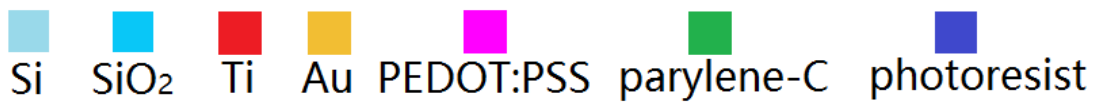

Figure 3.6: Fabrication process flow. (a) $\mathrm{Au}(50 \mathrm{~nm}) / \mathrm{Ti}(5 \mathrm{~nm}) / \mathrm{SiO}_{2}(250 \mathrm{~nm}) / \mathrm{Si}$ substrate. (b) Deposit PEDOT: PSS on the substrate and then zeolite crystals are transferred onto the substrate. (c) Evaporate 50-100 nm thick parylene-C. (d) Spin-coat the photoresist (Olin907/17)(1.7 $\mu \mathrm{m}$ thick) for the photolithography. (e) After exposure and developing, the photoresist layer is patterned. (f) Nanoindentation is performed in the area without photoresist covered. (g) $150 \mathrm{~nm}$ Au is evaporated on the sample everywhere. (h) Lift-off is executed to remove the unexposed photoresist and the Au on top of the photoresist.

Figure 3.6 shows the fabrication process flow in respect of the zeolite-crystal devices. At the beginning the zeolites have already been transferred onto conductive substrates, such as PEDOT: PSS/Au/Ti/SiO $/ 2$ Si substrates (Figs. 3.6(a) and (b)) or PEDOT: PSS/ITO substrates. 
After that, we firstly cover the whole sample with an insulating layer (Fig. 3.6(c)). As insulating layer we use parylene- $C$, which has good insulation when the thickness is larger than $50 \mathrm{~nm}$ and good surface flatness [30]. The coating of parylene-C is also conformal [31]. Then photolithography is executed on top of the parylene layer. The positive resist layer is Olin907/17 with $1.7 \mu \mathrm{m}$ thickness (Fig. 3.6(d)). After the photolithography, the areas where we want to implement the nanoindentation are opened up (Fig. 3.6(e)). After the nanoindentation, a hole is created right on top of a zeolite (Fig. 3.6(f)). To make the nanoindentation process not too difficult, we only deposit the parylene layer up to about $100 \mathrm{~nm}$. A layer of $150 \mathrm{~nm}$ Au is evaporated on the sample everywhere (Fig. 3.6(g)). The last step is lift-off to remove the unexposed resist and the Au on top of the resist to isolate the Au top contacts on top of the parylene layer (Fig. 3.6(h)).

In the fabrication process, the critical step is to accurately position nanoindents on the zeolites. Wider zeolites (larger diameters) usually signify longer channel length, that is, longer molecular wires inserted in the channels, and thus larger resistance of the molecular wires. Hence, we always sought for crystals with diameters of about $300 \mathrm{~nm}$ or smaller, to avoid that the device would have too high resistance. Taking this into account, accurate positioning of the nanoindentation is desired. As long as the nanoindentation hole was indented through to the bottom conducting substrate, the zeolites will be shorted. In the following we will show that this requirement can be fulfilled with fast nanoindentation technique using the "point-and-shoot" function of a Bruker Dimension Icon AFM [32].

In summary, we introduce a fabrication method based on nanoindentation using AFM for electron transport measurements on the zeolite $L$ crystals and two-dimensional electron systems in $\mathrm{LaAlO}_{3} / \mathrm{SrTiO}_{3}$ samples. In the following, results of nanoindentation performed using different AFM working modes are presented.

\subsection{Nanoindentation results and discussion}

3.4.1 Nanoindentation using conventional working modes of the Veeco Dimension 3100 AFM

In the following the nanoindentation using a Veeco Dimension 3100 AFM [33] will be discussed. We have tried several different methods and found that all of them did not satisfy our positioning requirements. 
Before executing nanoindentation on a zeolite, a zeolite with suitable size needs to be found by AFM scanning. Usually a zoom-in scan is necessary to center the small zeolites in the scanned areas. Once we have already centered a zeolite, the scanning is stopped and the tip goes back to the center of the scanning area and therefore is placed on top of the zeolite as well. After that, we start indenting in the sample by pressing the tip.

To scan the sample, two working modes can be used. First one is the tapping mode and the second is the contact mode. Tapping mode (TM) scanning requires a non-contact-mode AFM probe. If the tapping mode is chosen for the scanning, then we either directly indent in the tapping mode or switch to the TM deflection mode or the contact mode to do the nanoindentation. Similar to the contact mode, the TM deflection mode also adopts the deflection of laser instead of the amplitude as the feedback signal. Considering that switching to the contact mode could cause some unpredictable drift of the tip, we usually avoid to use the contact mode for the nanoindentation if the tapping mode is used for the scanning. As for the scanning in the contact mode, a contact-mode AFM probe is required. The contact-mode AFM probe has a softer cantilever (a longer cantilever, and a smaller force constant) than the non-contact-mode probe. After the scanning we directly start indentation without switching working mode. Shortly speaking, we have three conventional methods to do the nanoindentation, listed in the following table. We have tried all of the three methods and the results are discussed in the following.

Table 3.1: Three nanoindentation methods using the Veeco Dimension 3100 AFM

\begin{tabular}{|c|c|c|}
\hline & Scanning mode & Indentation mode \\
\hline 1 & Tapping & Tapping mode deflection \\
\hline 2 & Tapping & Tapping \\
\hline 3 & Contact & Contact \\
\hline
\end{tabular}

\section{Method 1:}

In this method, the scanning was performed in the tapping mode. The sample consisted of parylene-coated zeolites, which had already been transferred onto the conductive PEDOT: PSS/ITO substrates. The parylene thickness was about $100 \mathrm{~nm}$. The AFM probe is a conductive PtSi alloy with a $48 \mathrm{~N} / \mathrm{m}$ force constant. When the AFM tip was indenting in the parylene layer during the nanoindentation, a voltage was applied to the tip. An oscilloscope was used to monitor the current signal which is related to the tip-sample resistance, so that we knew when the tip had just indented through the insulating layer and when to retract 
the z-piezo to withdraw the tip out as well. Afterwards, the sample was scanned in the tapping mode again to check the nanoindentation result.
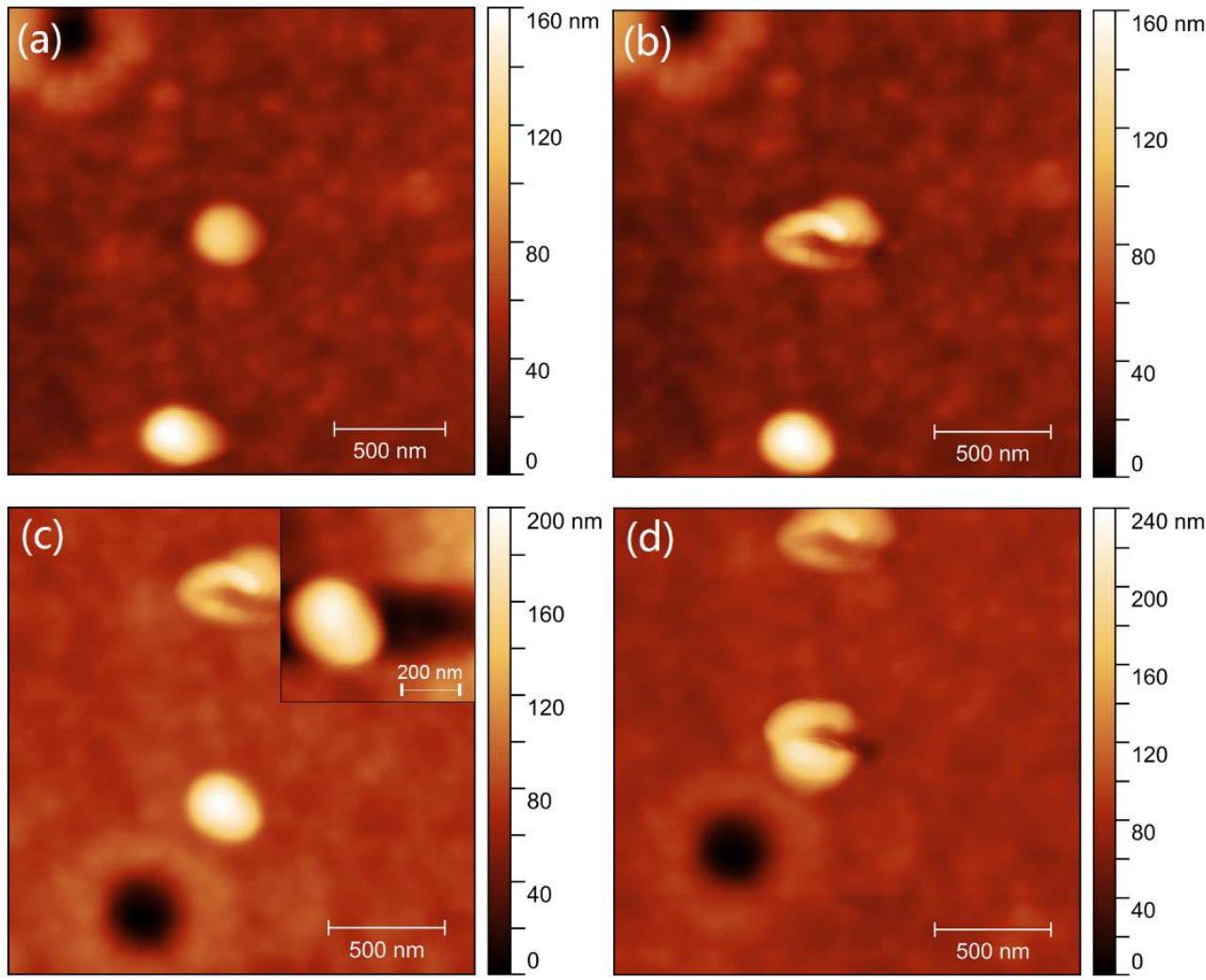

Figure 3.7: Nanoindentation results on parylene-coated zeolites using Method 1. (a) and (c) are AFM images scanned before the nanoindentation. (b) and (d) show the nanoindentation results of (a) and (c) respectively. Inset of (c) indicates that we stopped the tip at the right side of the zeolite, instead properly centered the zeolite.

Figure 3.7 shows two typical nanoindentation results using this method. Figures 3.7(a) and (c) are the AFM images of zeolites of about $300 \mathrm{~nm}$ diameter scanned before the nanoindentation which are shown in Figs. 3.7(b) and (d). From Fig. 3.7(b) we can see that the hole is shifted from the center of the zeolite where the hole was supposed to be. The offset magnitude is about $200 \mathrm{~nm}$. After many times of nanoindentation, we found that the final positions of the holes relative to the zeolites are different, although we had already centered the zeolites. We also checked if we could cancel out this offset by intentionally shifting the zeolites away from the center in the final scanned areas before nanoindentation. As shown in the final zoom-in AFM image of a zeolite in the inset of Fig. 3.7(c), we executed nanoindentation at the side of the zeolite. Nevertheless, eventually the hole was still not in the center of the zeolite. Results of Method 1 reveal that the nanoindentation holes were 
always created around zeolites with random offsets relative to the zeolites. From the morphology of the holes we also speculate that the tip not only vertically indented in the parylene, but also laterally scratched the parylene a little bit.

It is worth mentioning that if the zeolites are wider, for example, the diameter is larger than $500 \mathrm{~nm}$, then we will have better chance to create nanoindentation holes right on top of the zeolites. Overall speaking, this method is not a complete nightmare, if there is no such strict positioning requirement. Besides, in this way we can gradually increase the deflection set point and indent slowly, so that we can cautiously control the rest thickness of the insulating layer, which is similar to the work in Refs. [25, 26].

\section{Method 2:}

We also performed nanoindentation in the tapping mode after the tapping mode scanning. The starting point of this method was that directly executing nanoindentation without switching feedback signal could probably suppress the drift of the holes.

The AFM tips used in this method were still the PtSi tips used in Method 1. After centering the zeolite, we gradually decreased the amplitude set point to press the tip against the sample harder and harder. The sample was still parylene-coated zeolites on PEDOT: PSS/ITO substrates. The parylene thickness was about $100 \mathrm{~nm}$. We found that only when the amplitude set point became very small could the tip indent in the parylene layer, when decreasing the amplitude set point. Figures 3.8(a) and (b) show the AFM images before and after one nanoindentation test respectively. In that test, when the amplitude set point was not too small (larger than $0.02 \mathrm{~V}$ ), the tip was still oscillating and we did not see any conducting signal. When the set point reached $0.02 \mathrm{~V}$, the tip suddenly stopped oscillating and crashed into the sample. At the same time, we observed conducting signal in the oscilloscope. The AFM image (Fig. 3.8(b)) scanned after the nanoindentation reveals a very big hole and we could not identify the original zeolite.

We tried the nanoindentation on a test sample which was $95 \mathrm{~nm}$ parylene-C coated on $\mathrm{Au}$ substrate. The AFM probe was common silicon non-contact-mode probe. In one tapping mode scanning, $1.526 \mathrm{~V}$ amplitude set point was the largest value with which the tip could follow the topography of the sample surface very well. When we decreased the amplitude set point gradually to $0.1 \mathrm{~V}$, the tip was still oscillating and scanning the sample, shown in Fig. 3.8(c). We can already see that there are many error lines in the image, which signifies that the tip was pressed against the sample very hard under such small amplitude set point and thus disturbed by the sample surface during the scanning. When the set point was 
decreased further to $0.05 \mathrm{~V}$, the tip suddenly crashed into the sample. After withdrawing the tip and scanning again, we observed a big hole, as shown in Fig. 3.8(d).

In summary, using Method 2, if the amplitude set point is not set very small, AFM tip will not indent in the parylene layer. However, when the set point is very small, the tip immediately hits into the insulating layer and generates very big holes. This sudden crash is not constrained by any tunable set point. Hence, the height of holes cannot be controlled. In addition, this method is very destructive to the tip. The tip becomes too blunt to be used for scanning very soon.
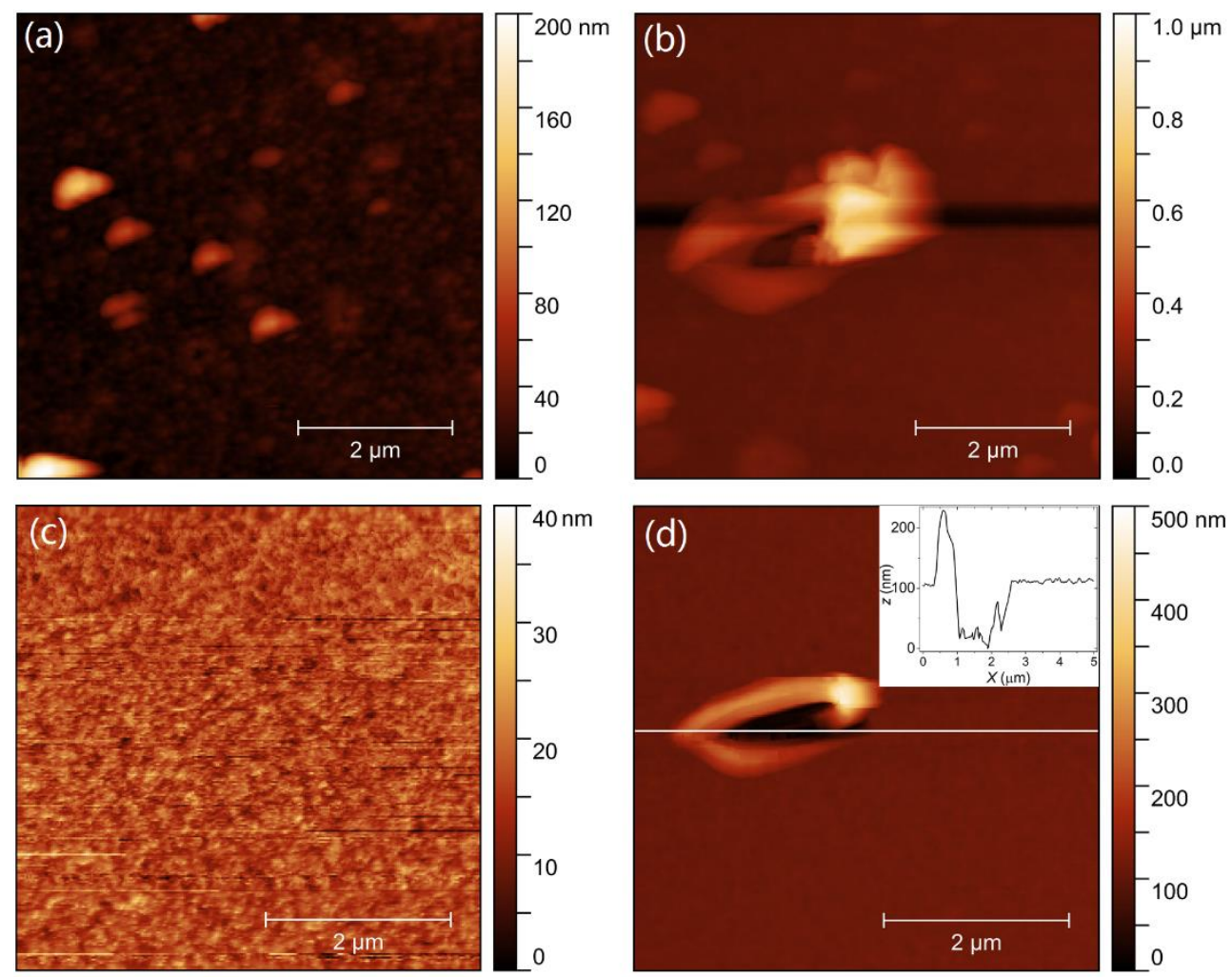

Figure 3.8: Nanoindentation results on parylene-coated zeolite and parylene-coated Au respectively, using Method 2. (a) and (c) are AFM images scanned before the nanoindentation. (b) and (d) are AFM images scanned after the nanoindentation of (a) and (c), respectively. Inset of (d) is the profile plot. 


\section{Method 3:}

In the last method, the contact mode was adopted for both scanning and indentation. After scanning to center zeolites, the tip was stopped at the center of the scanned area. Then we increased the deflection set point gradually to indent in the insulating layer.

Firstly, a PtIr conductive probe with a $1.87 \mathrm{~N} / \mathrm{m}$ force constant was used. During the scanning, a small $(0.2 \mathrm{~V})$ deflection set point was set. The test sample was $76 \mathrm{~nm}$ parylene coated on Au substrate. The tip was also biased to monitor conducting signal during the nanoindentation. Figure 3.9(a) is the scanned image. In the nanoindentation process, we did not observe conducting signal when increasing the deflection set point from $0.2 \mathrm{~V}$ to the maximum value $(10 \mathrm{~V})$. After that, the deflection set point was set back to $0 \mathrm{~V}$ immediately to withdraw the tip, and then indent in the same way again. After two times of this repeat, that is, three times of the nanoindentation in total, we still did not measure any conducting signal. Scanned AFM image after this shows that the "hole" looks more like a lateral scratch (Fig. 3.9(b)), compared to the previous nanoindentation results obtained in Method 1. And the scratch is also very long, compared to the diameters of the desired zeolites. The arrow points out the direction of the scratching on the surface, which is, from the center of the image where we stopped the tip, to the left side. The profile plot (Inset of Fig. 3.9(b)) reveals that the depth of the hole is only about $10 \mathrm{~nm}$, which indicates that the tip had not yet indented through the parylene layer at all after three times of the nanoindentation. This explains why we did not see any conducting signal in the oscilloscope. The failure to indent through the parylene layer is probably because the cantilever is too soft to withstand the counterforce from the sample surface.

Since the PtIr probe was probably too soft to execute nanoindentation properly in the contact mode, we also tried another contact-mode AFM probe with a larger force constant, $2.8 \mathrm{~N} / \mathrm{m}$. The material of the probe was the PtSi alloy mentioned in Method 1. The sample was still same as above. The contact mode scanning also required a small deflection set point (0.2 V). As shown in the AFM image scanned before nanoindentation (Fig. 3.9(c)), the tip already scratched the parylene surface during the scanning. After the scanning, we performed a nanoindentation process similar to one we executed when using the PtIr tip. This time, the difference was that we observed conducting signal in the second nanoindentation when the deflection set point was $9.5 \mathrm{~V}$. Scanned image after the nanoindentation (Fig. 3.9(d)) shows that the hole is still very long. The hole profile (Inset of Fig. 3.9(d)) indicates that the parylene layer had been indented through. From this nanoindentation test we can see that, using the stiffer probe, we still had to indent twice and apply big force to indent through the parylene layer. 

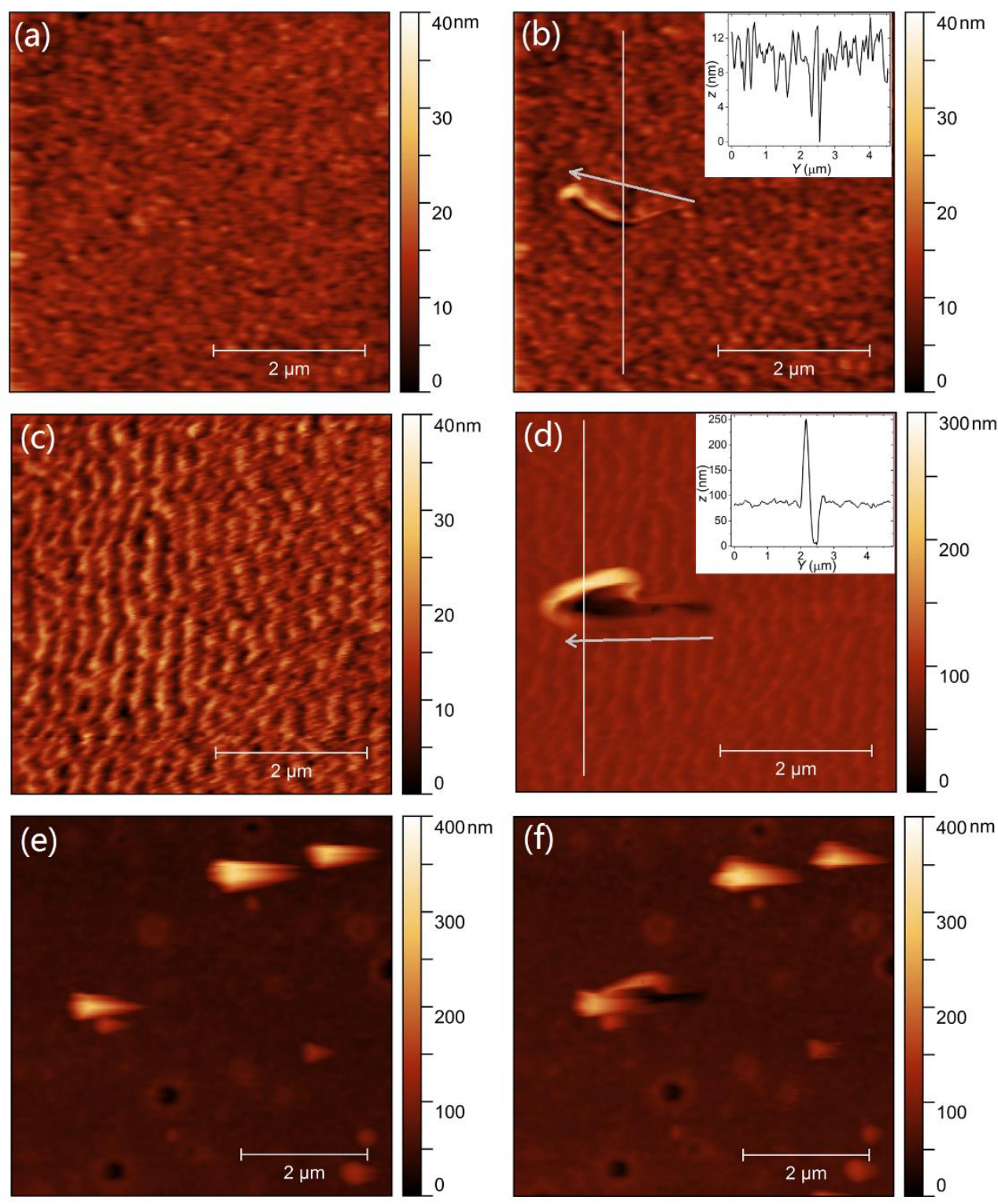

Figure 3.9: Nanoindentation results on parylene-coated zeolites and parylene-coated Au respectively, using Method 3. Left side are AFM images scanned before the nanoindentation. Right side are AFM images scanned after the nanoindentation. (a) and (b) are results of the parylene-Au test sample using the PtIr tip with a $1.87 \mathrm{~N} / \mathrm{m}$ force constant. (c) and (d) are results of the parylene-Au test sample using the PtSi tip with a $2.8 \mathrm{~N} / \mathrm{m}$ force constant. (e) and (f) are results of the parylene-zeolite sample using the PtSi tip. Insets of (b) and (d) are the profile plots. Arrows on (b) and (d) indicate the direction of the scratching of the AFM tips. 
This method had also been tried on the zeolite sample. The parylene thickness was about $100 \mathrm{~nm}$. During the scanning, we did not set the deflection set point too large to avoid crash of the tip onto the hundreds of $\mathrm{nm}$ high zeolites. Therefore, the probe could not completely trace the sharp slope of zeolites and we can see the "comet tail" effect in the AFM images (Figs. 3.9(e) and (f)). Besides this scanning problem, the more important problem is that the "hole" is too big for the small zeolites, shown in Fig. 3.9(f). To summarize, in Method 3, the contact-mode AFM probes are too soft to indent through the parylene layer. In addition, the nanoindentation holes are too big for the small zeolites.

Based on the results of Method 1 and 3, we propose that the offset of the holes is probably due to the lateral drift of AFM tips during the nanoindentation. Figure 3.10 is a schematic process showing this drift. Different colored probes stand for different statuses of an AFM probe in the nanoindentation process. No matter it is the TM deflection mode or the contact mode that is used for the nanoindentation, the deflection set point always needs to be increased gradually to execute the nanoindentation. Every time after increasing the deflection set point, the z-piezo engages the AFM probe towards the sample and thus the tip indents into the parylene. When the tip is indenting in the parylene layer, it is subject to a counter force exerted by the sample. This force causes upward bending of the cantilever, indicated by the pink and purple probes in Fig. 3.10, so the deflection signal increases. When the deflection signal reaches the set point, the piezo stops engaging and the indenting stops. Therefore, as the deflection set point is set larger and larger, the tip can indent deeper and deeper in the parylene. Due to the bending of the cantilever, eventually there will be a lateral movement of the tip, indicated by the dashed arrow in Fig. 3.10. And this movement is always pointing from the right to the left. This is why the parylene was always pushed from the right to the left, shown in Figs. 3.7 and 3.9. Furthermore, one can also imagine that the softer the cantilever, the more severe the bending, hence the longer the lateral movement, which explains why the soft contact-mode probes caused that long scratches in the parylene.

In conclusion, the three methods are all inappropriate for the nanoindentation to create holes right on top of zeolites with diameters of about $300 \mathrm{~nm}$ or even smaller. The problems are the positioning issue and the size of the nanoindentation holes. In respect of Method 3 , soft AFM probes are not suitable for indenting through the parylene layers. 


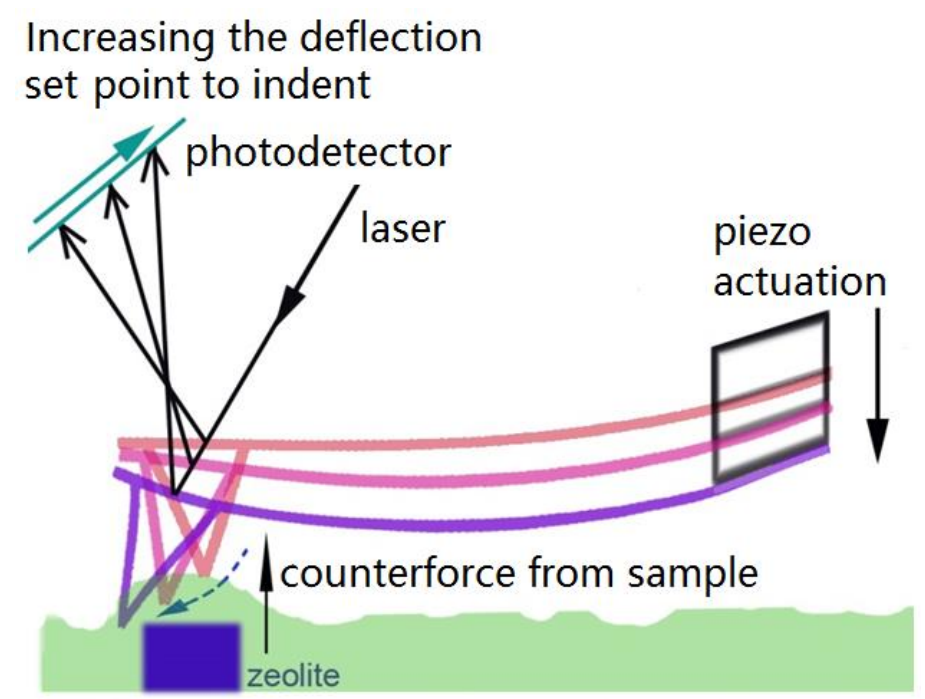

Figure 3.10: Schematic process showing how the tip is drifting laterally in the nanoindentation process using the TM deflection or the contact mode. Three different colors of AFM probes schematically indicate three different statuses of an AFM probe during the nanoindentation process.

3.4.2 Nanoindentation with accurate positioning using the "point-and-shoot" function

The AFM used for this part is a Bruker Dimension Icon AFM, which has a so-called "pointand-shoot" working mode. As mentioned above, in the TM deflection mode and the contact mode, the deflection set point is changed gradually in the nanoindentation process. In contrast, in the point-and-shoot mode, a deflection set point needs to be set only once at the beginning of a nanoindentation process. This set point is called the "trig threshold". During the nanoindentation process, the trig threshold is fixed. After setting the trig threshold, the voltage applied to the z-piezo-actuator quickly ramps up first, which actuates the tip to indent the sample fast. Then the voltage quickly ramps down when the laser deflection signal reaches the trig threshold, and the tip is withdrawn back quickly. This ramp loop is so short (much less than 1 second, if we do not set a delay time between the ramping up and down) that the tip seems to be "shooting" towards the sample.

The point-and-shoot nanoindentation process is schematically shown in Fig. 3.11. The left part shows the TM deflection-z curve of one nanoindentation process. The blue curve corresponds to the stage when the AFM tip is indenting, and the red curve corresponds to the stage when the tip is being withdrawn. The numbers along the curve correspond to the tip positions shown at the right. When the tip moves towards the sample, it is subject to an 
attractive force from the sample till it touches the sample (1 to 3). After that, the tip indents into the parylene layer, and the cantilever is bent upward, so the TM deflection increases (3 to 4). Once the TM deflection reaches the trig threshold (at 4), the piezo retracts to withdraw the tip, and the TM deflection starts decreasing as the cantilever restores from the upward bending position. However, when the cantilever recovers back to its straight position, the tip is still stuck in the parylene layer. Therefore, as the z-piezo continues the retraction, the cantilever starts to bend downward. And the lowest point of the TM deflection is lower than the initial position (at 5). At that moment, the cantilever has the maximum downward bending. Immediately after that, the tip leaves the parylene layer quickly. Finally, the TM deflection returns back to its initial position as the cantilever restores (5 to 6).

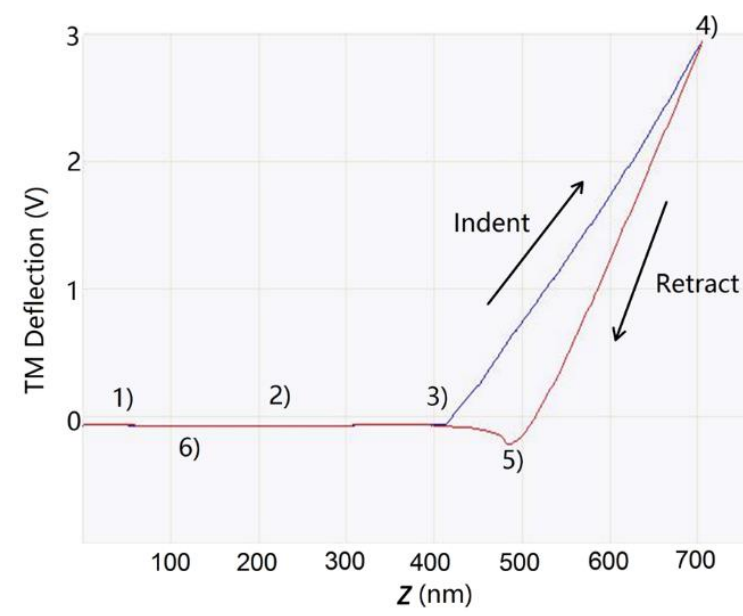

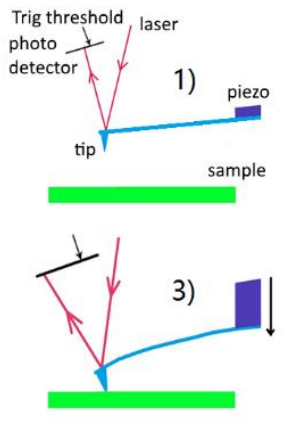
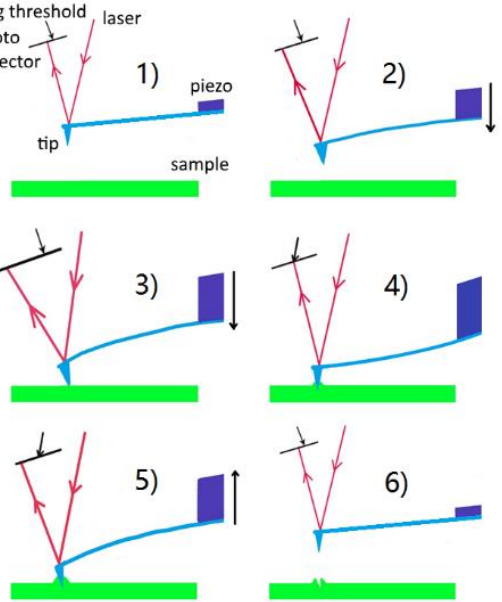

Figure 3.11: Schematic nanoindentation process in the point-and-shoot mode. Left: Experimental TM deflection$z$ curve of one nanoindentation process. Right: Tip position corresponding to the numbers in the left image.

Figure 3.12 demonstrates a typical nanoindentation result using the point-and-shoot function. The sample was zeolites covered by about $90 \mathrm{~nm}$ parylene. The AFM images were scanned before and after the nanoindentation respectively. The dashed circles on the AFM images indicate the position of the zeolite. The result shows that the nanoindentation hole is exactly on top the zeolite. The morphology of the hole (Fig. 3.12(b)) also suggests that the tip mainly indented vertically in the sample, instead of laterally scratched over the zeolite. Comparing the profile plots, we can estimate the hole depth. In this case, the hole depth is about $100 \mathrm{~nm}$. In this method, we have more than $90 \%$ chance to put a hole right on top of the small zeolites with diameters of about $150-300 \mathrm{~nm}$. 

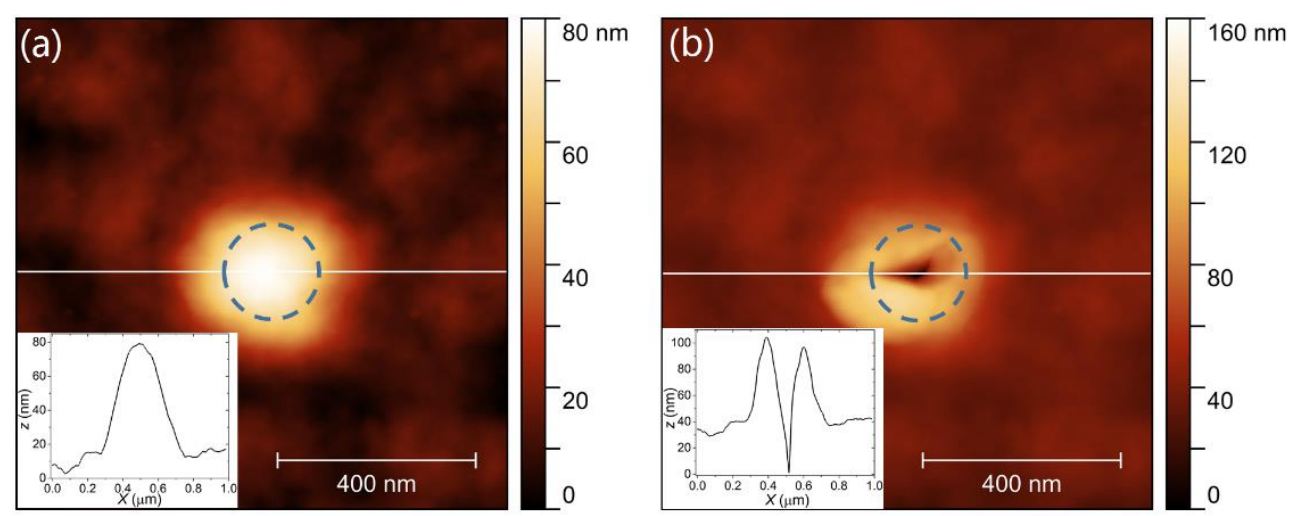

Figure 3.12: Nanoindentation result using the point-and-shoot mode. (a) and (b) are AFM images scanned before and after the nanoindentation respectively. Insets are the profile plots. Blue dashed circles on the AFM images are used to indicate the position of the zeolite.

We also tried to change several parameters of the ramping process to modulate the depth of holes. First parameter is the trig threshold mentioned before. As we described in above, by increasing the trig threshold, we expected deeper holes. The second parameter is the delay time which determines for how long the z-piezo actuator keeps its maximum expanding state before retracting. As we know, when the tip is indenting in the parylene layer, the cantilever is bending upwards. Therefore, there should be some elastic energy stored in the cantilever due to this bending. If the tip is not withdrawn immediately, it probably will be able to indent deeper. Hence, we also expected deeper holes by increasing the delay time. The third parameter is the shoot times at same places, that is, perform multiple nanoindentation processes at the same points. As shown in the top red square of Fig. 3.14, the ramping times was set as 3 . We expected deeper holes by increasing the shoot times.

Figure 3.13(a) shows the trig threshold dependence of the hole depth. The delay time was fixed at three different values, 0,1 and $3 \mathrm{~s}$. We can see that as we increase the trig threshold, the hole depth increases. However, there is no clear rising trend in the hole depth with the delay time, which suggests that such short delay does not cause significant change to the hole depth. From Fig. 3.13(b) we can see that when we indent more times at the same point, the hole depth increases. And the hole depth is generally larger when using $1.5 \mathrm{~V}$ trig threshold than using $1 \mathrm{~V}$ trig threshold. 

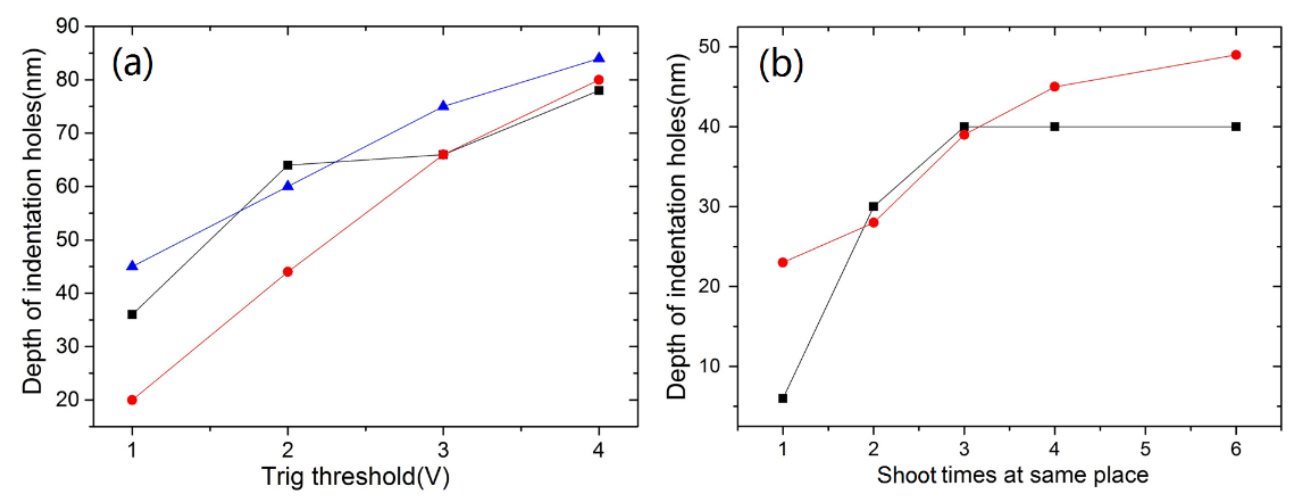

Figure 3.13: Hole depth vs. ramping parameters in the point-and-shoot mode. (a) Depth of the holes as a function of the trig threshold. The delay time was set to $0 \mathrm{~s}$ (black square), $1 \mathrm{~s}$ (red dot), $3 \mathrm{~s}$ (blue triangle), and always indented one time. (b) Depth of the holes as a function of the shoot times at same place. The trig threshold was set to $1 \mathrm{~V}$ (black square) and $1.5 \mathrm{~V}$ (red dot). The delay time was always set to 0.

Sometimes we would like to expand the nanoindentation hole to ensure that deposited metal in the hole contacts well with the bottom sample. In the literature a short oxygen plasma was used to clean and enlarge the hole at the same time [29]. If the sample under the hole is sensitive to the plasma treatment, then the oxygen plasma method is probably not appropriate. In this case, we can indent in a way called "box of shooting points". Simply speaking, we just need to set multiple indentation points arranged in a 2-dimensional array with some spacing between each two neighbor points in the row and column directions. Thereby, we expected to be able to make a bigger nanoindentation hole. Figure 3.14 is the operation interface of the point-and-shoot function. The bottom red square shows where one can set the indentation point array. In the figure, a $4 \times 4$ array was set. This 2 -dimensional indentation point array is also shown in the red circle of the figure.

Figure 3.15 demonstrates one nanoindentation result using this kind of setting. The trig threshold was $3 \mathrm{~V}$, the delay time was 0 , and the tip only indented one time at each point. A $2 \times 2$ array with $10 \mathrm{~nm}$ spacing in both directions was adopted. The profile plot of Fig. 3.15(b) shows that the nanoindentation hole has a wider bottom part, compared to that of Fig. 3.12(b). 


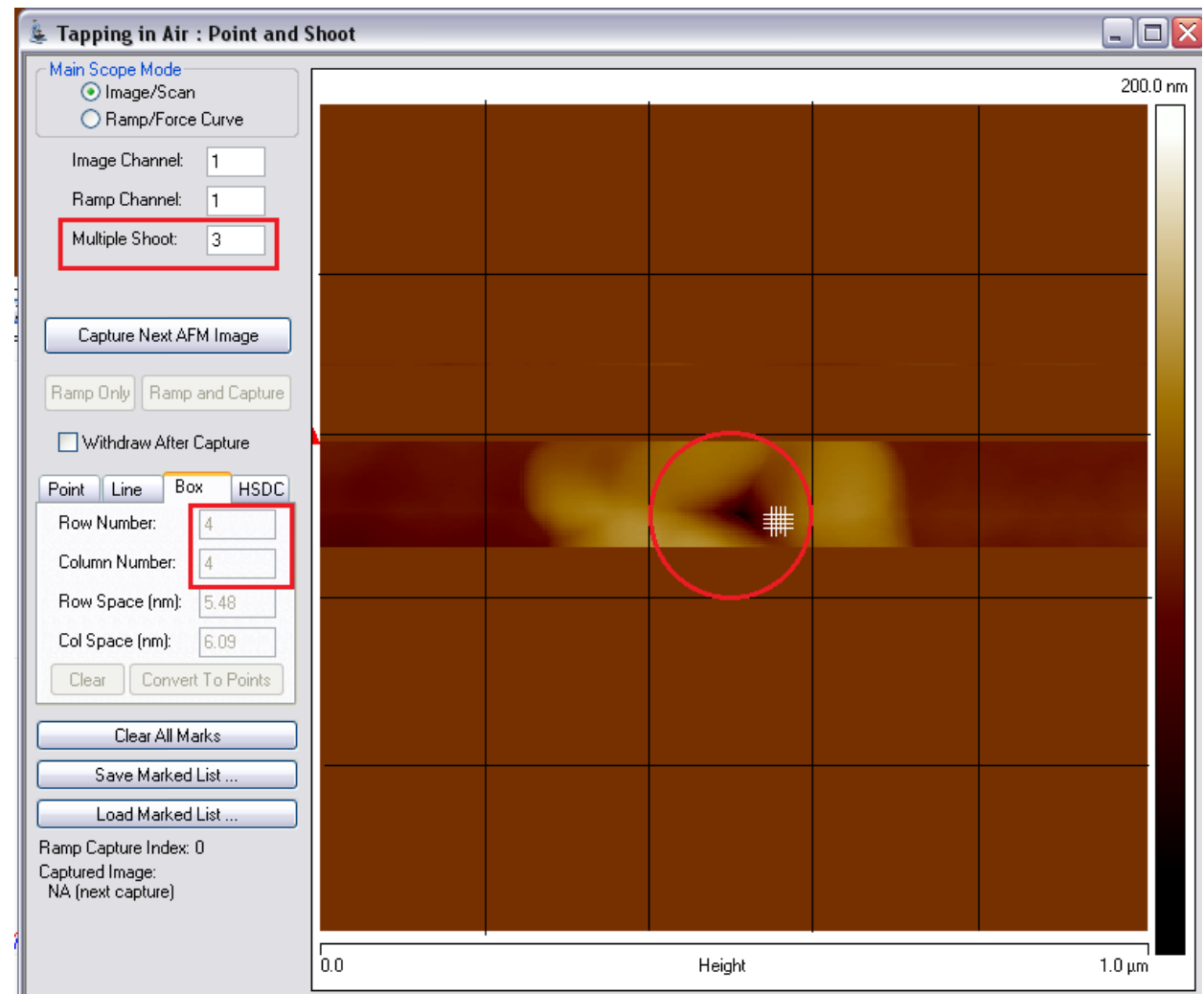

Figure 3.14: Screenshot of the user interface of the point-and-shoot mode. The top red box is where to set the shoot times. The bottom red box shows the row and column settings for the 2-dimensional indentation point array. The red circle indicates the position of the zeolite.
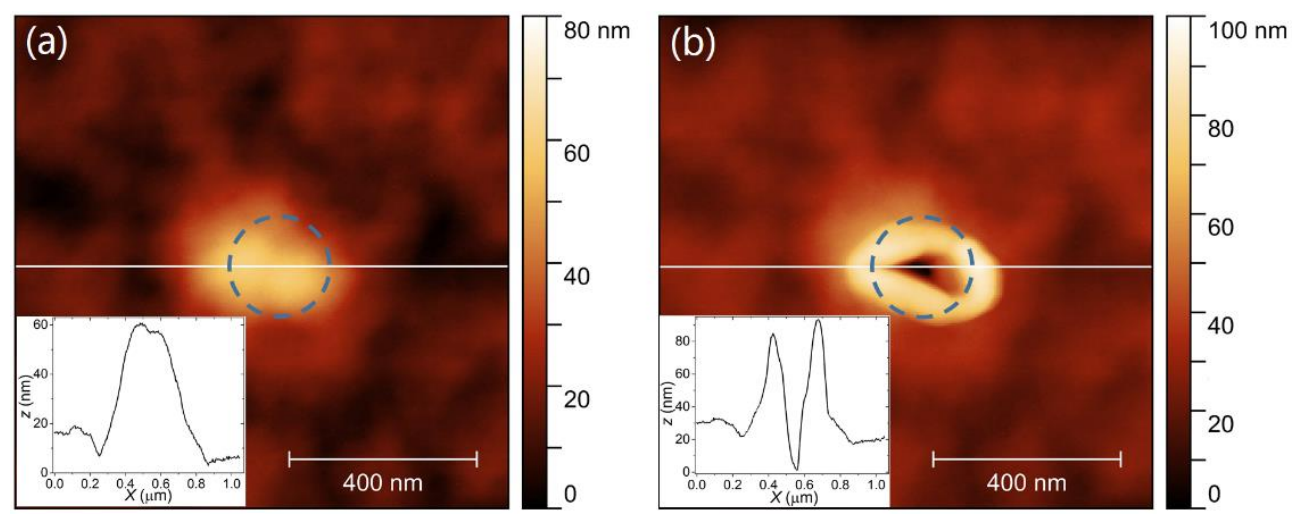

Figure 3.15: Nanoindentation result by setting a 2-dimensional indentation point array. (a) and (b) are AFM images scanned before and after the nanoindentation respectively. Insets are the profile plots. Blue dashed circles on the AFM images are used to indicate the position of the zeolite. 
CP-AFM nanoindentation was executed as well to monitor current signal. We used a parylene $(65 \mathrm{~nm}) / \mathrm{Au}$ test sample. The conductive probe was biased at $5 \mathrm{mV}$ during the nanoindentation. Since the contact time between the tip and bottom Au is very short in the point-and-shoot mode, the current signal detected by the oscilloscope should be instant pulses. With the help of the current monitoring, we accurately knew whether the tip had indented through the parylene layer. The CP-AFM nanoindentation results are shown below.

When the above-mentioned "box of shooting points" method is used to perform the nanoindentation, it is good to know when the tip just indents through the parylene layer, thereby knowing roughly if the bottom of the hole is enlarged. For example, if multiple shooting is set to perform a nanoindentation, but only one current pulse is observed in the end of the nanoindentation process, then the parylene layer is actually just indented through by that one shoot. This means that the bottom of the hole is probably not as large as we expect, and that we need to adjust the shooting parameters to make the tip indent harder. Hence, we performed the nanoindentation using the "box of shooting points" method and used the oscilloscope to observe when the parylene was indented through. We set a $4 \times 4$ array of indentation points with small spacing $(\sim 5 \mathrm{~nm})$ in the row and column directions, 3 shoot times at each point, as shown in Fig. 3.14. The delay time was $500 \mathrm{~ms}$. By this setting, we expected to observe 48 instant pulses if the tip could indent through the parylene and touch the bottom Au substrate in every shooting process. Figure 3.16(a) is the screen capture of the oscilloscope during the nanoindentation. The yellow line is the measurement channel. As shown in the figure, only two current pulses appeared when the nanoindentation was almost finished. This suggests that the tip had not indented through the parylene layer yet before the last two shootings, which was probably because a relative small trig threshold (1.8 V) was set. As the multiple-time shooting kept going on, the tip indented deeper and deeper and eventually indented through the insulating layer and touched the bottom Au substrate. When the trig threshold was increased to $2.5 \mathrm{~V}$, we observed more pulses, as shown in Fig. 3.16(b). The result implies that the tip indented through the parylene layer earlier than the first experiment. In another test, we set 0 delay time. We observed shorter time duration of the pulses than the previous two tests, as shown in Fig. 3.16(c). To conclude, by performing the CP-AFM nanoindentation, we are able to know whether/when the tip indents through the insulating layer.

After the nanoindentation step, we finished the fabrication process shown in Fig. 3.4 and obtained Au-parylene-Au test devices. We measured their current-voltage properties. All the devices with nanoindentation holes in the parylene layers show very conductive properties. The $I V$ characteristic of Fig. 3.16(d) shows a small resistance which is about 50 $\Omega$. The $50 \Omega$ resistance is due to a series resistor used in the $I V$ measurement setup. In 
contrast, if there was no nanoindentation executed, the eventual devices show a very large resistance which is about $70 \mathrm{G} \Omega$ according to the slope of the IV loop (Fig. 3.16(e)), which indicates that the $65 \mathrm{~nm}$ parylene has good insulation. The hysteresis loop is probably due to the charging and discharging effect of the insulating devices during the voltage sweeping.

\subsection{Summary and outlook}

To perform electron transport measurements on one-dimensional DXP-loaded zeolite L crystals and two-dimensional electron systems in LAO/STO heterostructures, we introduce a fabrication method based on nanoindentation using atomic force microscopy. Parylene-C is used as the indent material in the fabrication method. To verify the positioning property of the nanoindentation, we tried three common methods from literature and the "pointand-shoot" mode to perform nanoindentation on top of. We found that the common methods did not meet the positioning requirement. Nanoindentation using the "point-andshoot" function showed good positioning properties, and enabled to create holes on top of the small zeolites. The depth and size of the holes was controlled by adjusting the parameters of the "point-and-shoot" mode. IV measurements of the Au-parylene-Au test devices with nanoindentation showed high conduction. Test devices without nanoindentation showed good insulation. Devices containing DXP-loaded zeolites and LAOSTO devices with nanocontacts are to be fabricated. 

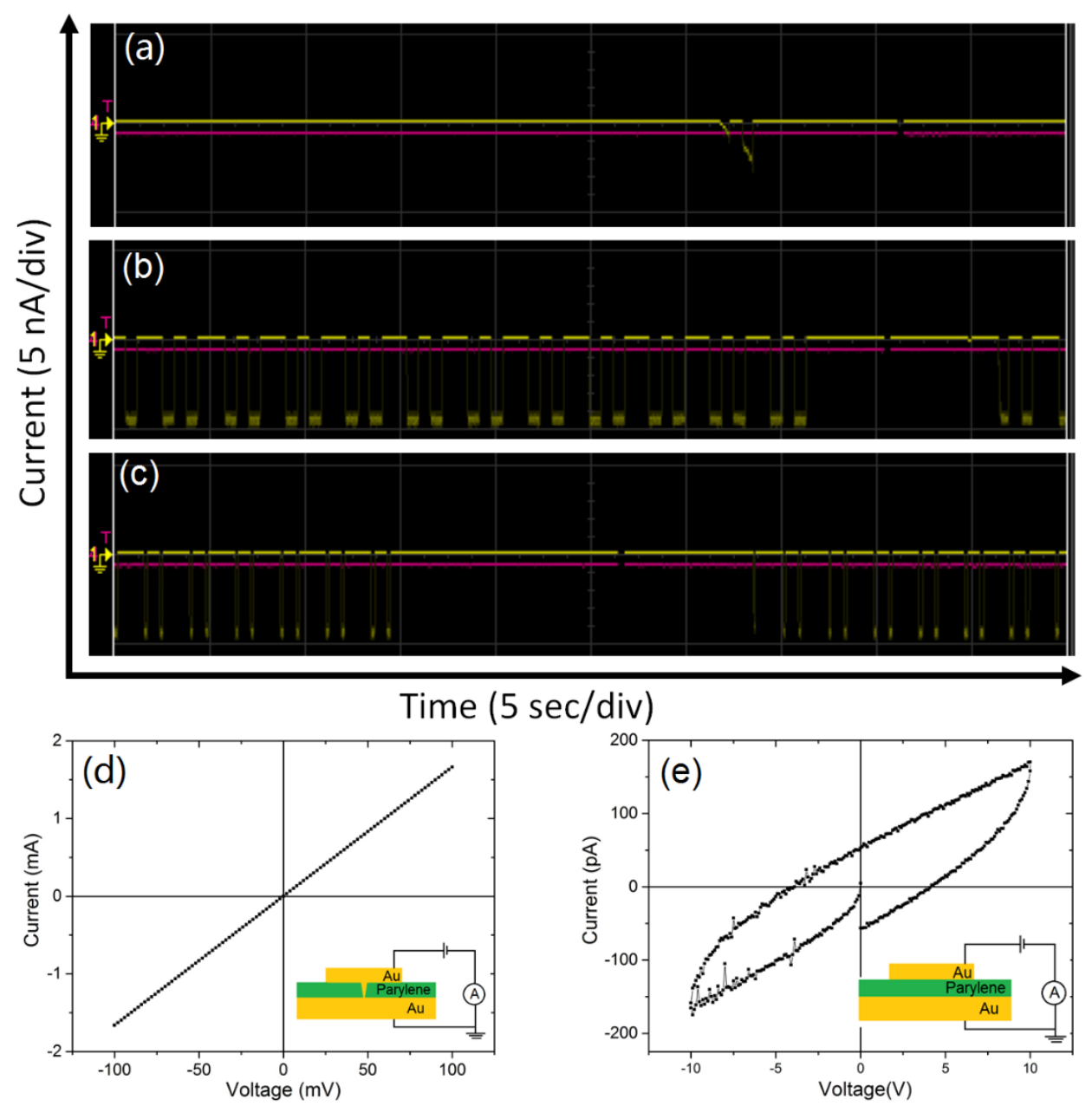

Figure 3.16: CP-AFM nanoindentation. (a), (b) and (c) Oscilloscope screen captures when monitoring the current signal during the nanoindentation. (a) Only two pulses were observed. Point-and-shoot parameters: $1.8 \mathrm{~V}$ trig threshold, $4 \times 4$ indentation point array, 3 shoot times at each point and $500 \mathrm{~ms}$ delay. The row and column spacings were both about $5 \mathrm{~nm}$. (b) More pulses were observed. Point-and-shoot parameters: $2.5 \mathrm{~V}$ trig threshold, $4 \times 4$ indentation point array, 2 shoot times at each point and $500 \mathrm{~ms}$ delay. The row and column spacings were both about $5 \mathrm{~nm}$. (c) Shorter time duration of the pulses than (a) and (b) was observed when 0 delay time was set. Other point-and-shoot parameters: $3 \mathrm{~V}$ trig threshold, $4 \times 4$ indentation point array, 2 shoot times at each point. (d) Typical $I V$ measurement result of the Au-parylene-Au test devices with nanoindentation holes. (e) Typical IV measurement result of the Au-parylene-Au test devices without nanoindentation. Insets of (d) and (e) schematically show the device structures and the $I V$ measurement setup. 


\section{References}

1. Ruiz, A.Z., et al., Synthesis of zeolite L. Tuning size and morphology. Monatshefte für Chemie/Chemical Monthly, 2005. 136(1): p. 77-89.

2. Bornhauser, P. and G. Calzaferri, Ring-opening vibrations of spherosiloxanes. The Journal of Physical Chemistry, 1996. 100(6): p. 2035-2044.

3. Calzaferri, G., et al., Host-guest antenna materials. Angewandte Chemie International Edition, 2003. 42(32): p. 3732-3758.

4. Busby, M., et al., Interactions of perylene bisimide in the one-dimensional channels of zeolite L. The Journal of Physical Chemistry C, 2011. 115(13): p. 5974-5988.

5. Huber, S. and G. Calzaferri, Energy transfer from dye-zeolite L antenna crystals to bulk silicon. ChemPhysChem, 2004. 5(2): p. 239-242.

6. Lee, S.K., et al., Electrochemistry, spectroscopy and electrogenerated chemiluminescence of perylene, terrylene, and quaterrylene diimides in aprotic solution. Journal of the American Chemical Society, 1999. 121(14): p. 3513-3520.

7. Lee, J., et al., Ultrasound - Aided Remarkably Fast Assembly of Monolayers of Zeolite Crystals on Glass with a Very High Degree of Lateral Close Packing. Advanced Materials, 2005. 17(7): p. 837-841.

8. Cucinotta, F., et al., Microcontact transfer printing of zeolite monolayers. Advanced Materials, 2009. 21(10 - 11): p. 1142-1145.

9. Ohtomo, A. and H. Hwang, A high-mobility electron gas at the LaAlO3/SrTiO3 heterointerface. Nature, 2004. 427(6973): p. 423-426.

10. Breitschaft, M., et al., Two-dimensional electron liquid state at LaAlO 3-SrTiO 3 interfaces. Physical Review B, 2010. 81(15): p. 153414.

11. Copie, O., et al., Towards two-dimensional metallic behavior at LaAlO 3/SrTiO 3 interfaces. Physical review letters, 2009. 102(21): p. 216804.

12. Reyren, N., et al., Superconducting interfaces between insulating oxides. Science, 2007. 317(5842): p. 1196-1199.

13. Richter, C., et al., Interface superconductor with gap behaviour like a hightemperature superconductor. Nature, 2013. 502(7472): p. 528-531.

14. Caviglia, A., et al., Tunable Rashba spin-orbit interaction at oxide interfaces. Physical review letters, 2010. 104(12): p. 126803.

15. Brinkman, A., et al., Magnetic effects at the interface between non-magnetic oxides. Nature materials, 2007. 6(7): p. 493-496.

16. Lee, J.-S., et al., Titanium $d x y$ ferromagnetism at the LaAlO3/SrTiO3 interface. Nature materials, 2013. 12(8): p. 703-706.

17. Xie, Y., et al., Control of electronic conduction at an oxide heterointerface using surface polar adsorbates. Nature communications, 2011. 2: p. 494.

18. Cen, C., et al., Nanoscale control of an interfacial metal-insulator transition at room temperature. Nature materials, 2008. 7(4): p. 298-302.

19. Wu, S., et al., Nonvolatile resistive switching in Pt/LaAlO 3/SrTiO 3 heterostructures. Physical Review X, 2013. 3(4): p. 041027.

20. Roos, W., R. Bruinsma, and G. Wuite, Physical virology. Nature Physics, 2010. 6(10): p. 733-743. 
21. Sattler, K.D., Handbook of nanophysics: functional nanomaterials. 2010: CRC Press.

22. Bishop, R., R. Hill, and N. Mott, The theory of indentation and hardness tests. Proceedings of the Physical Society, 1945. 57(3): p. 147.

23. Bulychev, S., et al., Determining Young's modulus from the indenter penetration diagram. Zavod. Lab, 1975. 41(9): p. 1137-1140.

24. Nix, W.D., Elastic and plastic properties of thin films on substrates: nanoindentation techniques. Materials Science and Engineering: A, 1997. 234: p. 37-44.

25. Fusil, S., et al., Nanolithography based contacting method for electrical measurements on single template synthesized nanowires. Nanotechnology, 2005. 16(12): p. 2936.

26. Bouzehouane, K., et al., Nanolithography based on real-time electrically controlled indentation with an atomic force microscope for nanocontact elaboration. Nano Letters, 2003. 3(11): p. 1599-1602.

27. Bernand-Mantel, A., et al., Evidence for spin injection in a single metallic nanoparticle: a step towards nanospintronics. Applied physics letters, 2006. 89(6): p. 062502.

28. Barraud, C., et al., Unravelling the role of the interface for spin injection into organic semiconductors. Nature Physics, 2010. 6(8): p. 615-620.

29. Galbiati, M., et al., Unveiling Self - Assembled Monolayers' Potential for Molecular Spintronics: Spin Transport at High Voltage. Advanced Materials, 2012. 24(48): p. 6429-6432.

30. Kahouli, A., Effect of film thickness on structural, morphology, dielectric and electrical properties of parylene C films. Journal of Applied Physics, 2012. 112(6): p. 064103.

31. Lee, H. and J. Cho. Development of conformal PDMS and parylene coatings for microelectronics and MEMS packaging. in ASME 2005 International Mechanical Engineering Congress and Exposition. 2005. American Society of Mechanical Engineers.

32. Bruker Dimension Icon AFM, https://www.bruker.com/products/surface-anddimensional-analysis/atomic-force-microscopes/dimension-icon/overview.html. (Date of access: 28-12-2016)

33. Veeco Dimension 3100 AFM, https://amolf.nl/veeco-dimension-3100-afm. (Date of access: 28-12-2016) 


\section{Chapter 4}

\section{DXP lateral field-effect transistors}

\subsection{Introduction and motivation}

Lateral field-effect transistor (FET) devices provide a convenient method to study charge transport in organic semiconductors [1]. We planned to investigate the charge transport properties of DXP in such lateral FET devices, in addition to the DXP-loaded zeolites described in Chapter 3. By investigating lateral FETs with a channel length similar to the height (sub-100 to hundreds of $\mathrm{nm}$ ) of the zeolites, we could compare results between the lateral devices and the zeolites. Magnetic resonance experiments, such as electron spin resonance (ESR) and electrically detected magnetic resonance (EDMR), are necessary to identify the spin states and spin species of charge carriers and to prove suggested theoretical models [2-6]. Coplanar waveguides have already been used to detect and control single spins in silicon single-electron transistors [7]. Hence, we also planned to combine the lateral devices with coplanar waveguides to perform EDMR studies of spin-dependent charge transport in the DXP devices.

In this chapter, electrical transport measurements of lateral DXP FETs are reported. The DXP FETs consist of DXP organic films deposited on Au electrodes pre-fabricated on $\mathrm{SiO}_{2} / \mathrm{Si}(\mathrm{p}++)$ substrates. To increase the conductance of the DXP devices, we fabricated interdigitated electrodes (IDEs) with short channels of about $100 \mathrm{~nm}$ channel length. Taking into account the short channel length, the $\mathrm{SiO}_{2}$ thickness was chosen to be $100 \mathrm{~nm}$, to enhance gate action.

\subsection{Experiments and Results}

\section{DXP spin-coating/drop-casting on $\mathrm{SiO}_{2}$ substrates}

DXP thin films were prepared from DXP solutions in 1,2-dichlorobenzene $(1.5 \mathrm{mg} / \mathrm{ml})$ using either spin-coating or drop-casting on $\mathrm{SiO}_{2}$ substrates. The solution was stirred at about $120^{\circ} \mathrm{C}$ for several hours prior to spin-coating. 1,2-Dichlorobenzene was chosen because of 


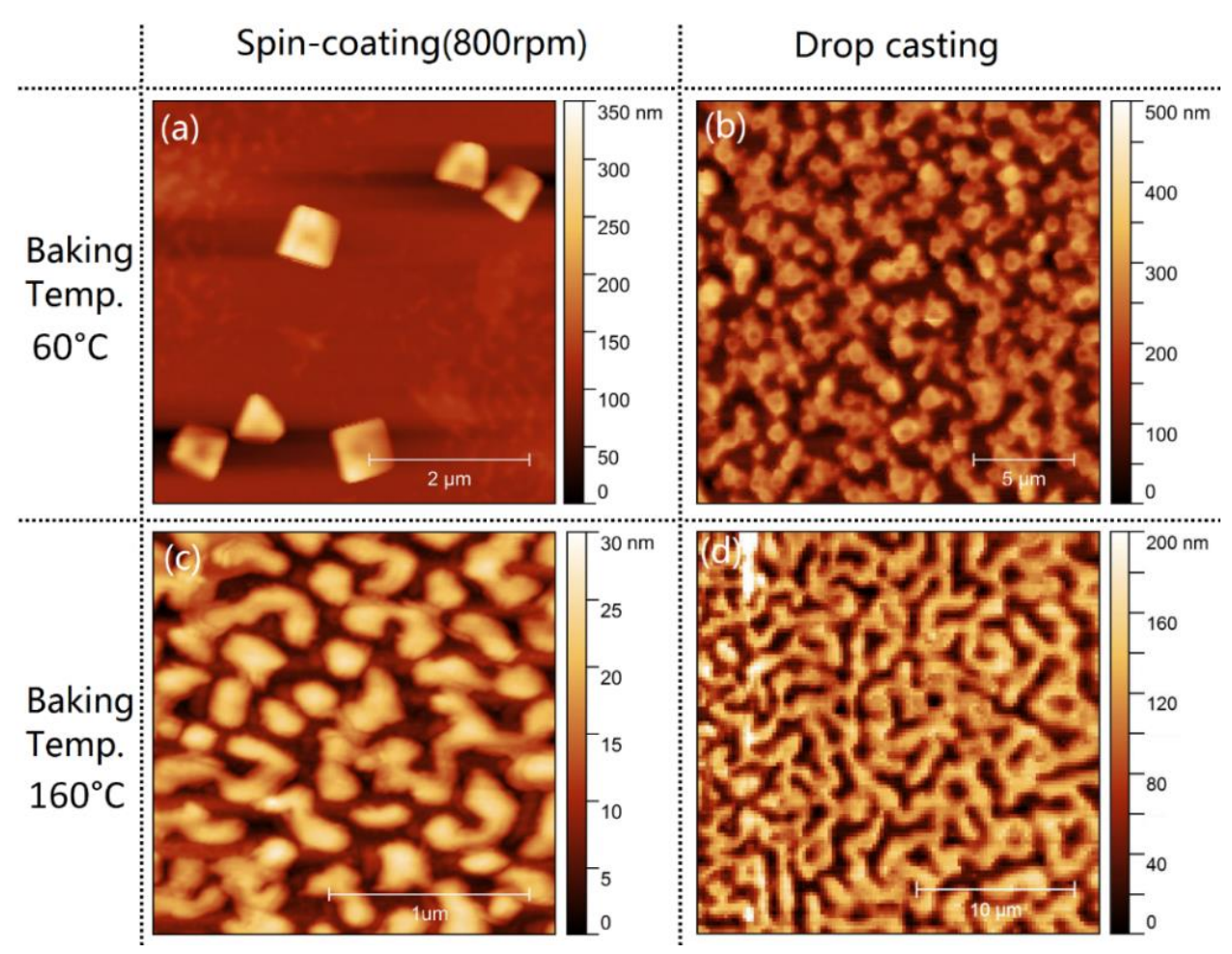

Figure 4.1: AFM images of DXP spin-coated and drop-casted on $\mathrm{SiO}_{2}$ substrates. (a), (c) are the spin-coating results and (b), (d) are the drop-casting results. (a), (b) were baked at $60^{\circ} \mathrm{C}$ and (c), (d) were baked at $160^{\circ} \mathrm{C}$.

its high boiling point $\left(180^{\circ} \mathrm{C}\right)$ so that we could heat up the solution to $120^{\circ} \mathrm{C}$ for extended time periods without evaporating the solvent. Before spin-coating, the $\mathrm{SiO}_{2}$ substrates were cleaned using acetone and isopropanol successively, followed by a short ( $1 \mathrm{~min}$ ) $\mathrm{O}_{2}$ plasma treatment. After spin-coating, the samples were baked on a hot plate at several different temperatures. Figures 4.1(a) and (c) show AFM images of two spin-coated films baked at $60{ }^{\circ} \mathrm{C}$ and $160{ }^{\circ} \mathrm{C}$, respectively. The observed morphology demonstrates that the DXP molecules formed crystallites rather than uniform films on the substrate when the solution was dried, especially after being baked at a lower temperature. One the one hand, crystallization is beneficial for obtaining high charge carrier mobility. On the other hand, when crystallization leads to discontinuity of the films, FETs cannot be produced reliably. hexamethyldisilazane (HMDS) treatment of the substrate did not introduce a significant change, which shows that wettability of the surface did not play a major role. Filtration of the solution with $0.2 \mu \mathrm{m}$ filters excluded the possibility the crystallites were pre-formed in the solution. It can thus be concluded that the DXP molecules self-organize and form 
crystallites as the solvent evaporates, similar to many other molecular organic semiconductors [8, 9]. Drop-casting was adopted afterwards, as it is an easier process that moreover enabled an increased coverage of DXP on the substrates, as shown in Fig. 4.1(d).

\section{Electrodes}

We firstly used single-gap Au electrodes to fabricate FETs. Figures 4.2(a) and (b) show AFM images of $\mathrm{Au}$ source and drain electrodes (with $\mathrm{Ti}$ adhesion layer) fabricated on a $\mathrm{SiO}_{2} / \mathrm{Si}$ substrate via electron beam lithography (EBL) and lift-off processes, before and after dropcasting DXP. Prior to drop-casting, the Au electrodes were cleaned using $\mathrm{O}_{2}$ plasma ( $20 \mathrm{~s}$ ) followed by an ethanol rinse, to remove residual organics left after the fabrication processes and oxides generated during the plasma stage, respectively [10]. The highly-doped silicon substrate was used as back gate electrode. The resulting device was measured at room temperature (RT), in a vacuum of $\sim 10^{-5}$ mbar obtained using a turbomolecular pump connected to a probe-station. The measurements were performed using a Keithley 2400 SourceMeter. We measured IV characteristics using source-drain voltages up to $10 \mathrm{~V}$, and found that the device was not conducting, as shown in Fig. 4.2(c). Back gate voltages up to $\pm 50 \mathrm{~V}$ were applied without significant change.
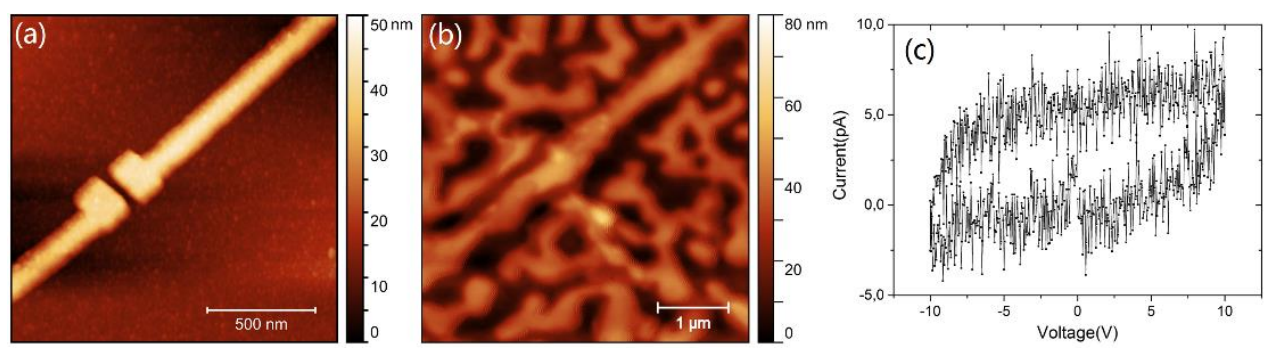

Figure 4.2: Results of one single-gap electrode device. (a), (b) AFM images of the ( $3 \mathrm{~nm} \mathrm{Ti/30} \mathrm{nm} \mathrm{Au})$ source and drain electrodes, separated by a gap of ... $\mathrm{nm}$, scanned before and after drop-casting DXP, respectively. (c) Current vs. voltage characteristic on the device shown in (b).

During IV measurements of the devices with short spacing (about 50-100 nm) between the source and drain electrodes, the maximum voltage was limited up to $15 \mathrm{~V}$ to prevent electromigration of the Au metal, which may cause measurement artifacts. Electromigration can break very narrow metallic constrictions [11, 12], thereby causing undesired open circuits. It can also bridge the narrow spacing between the electrodes and cause undesired 
shorts [13, 14]. The AFM images in Fig. 4.3 show an example of the result of electromigration after sweeping the source-drain voltage over a +/- $15 \mathrm{~V}$ range. The $I V$ in Fig. 4.3(b) recorded during this sweep shows clear current transients associated with the electromigration. Hence, we always checked all fabricated electrodes (after inspection with an optical microscope) for robustness upon $15 \mathrm{~V}$ or $10 \mathrm{~V} I \mathrm{~V}$ sweeps, depending on the gap size, before deposition of DXP. After the deposition, we only measured those devices which were previously checked to be completely insulating. In addition, during the transport measurements after the DXP deposition, we did not use voltages exceeding those used in the robustness tests to avoid possible electromigration in the small gaps.
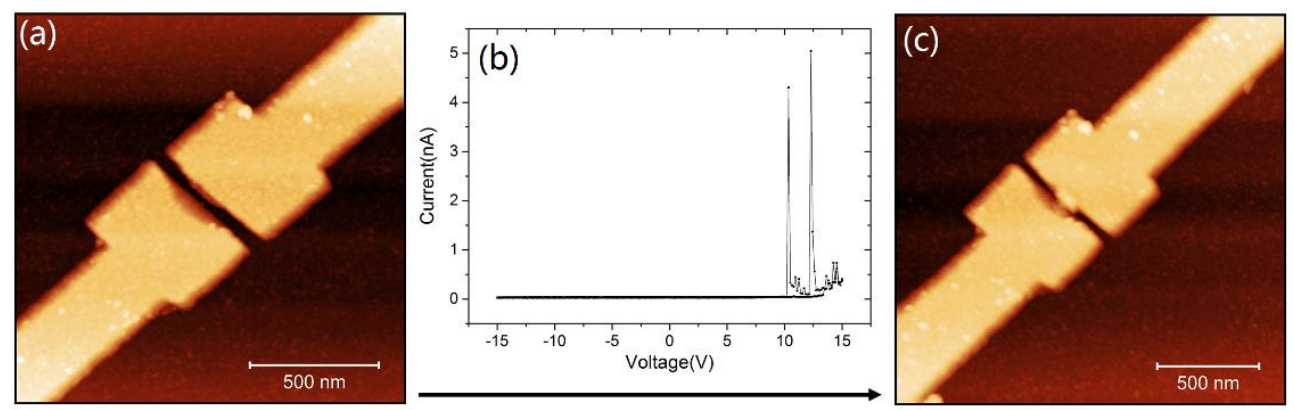

Figure 4.3: Electromigration of a small-gap electrode (without DXP coated) after ramping up to 15 V. (a), (c) AFM images of the electrode before and after a $15 \mathrm{~V} I V$ measurement, respectively. (b)The IV measurement.
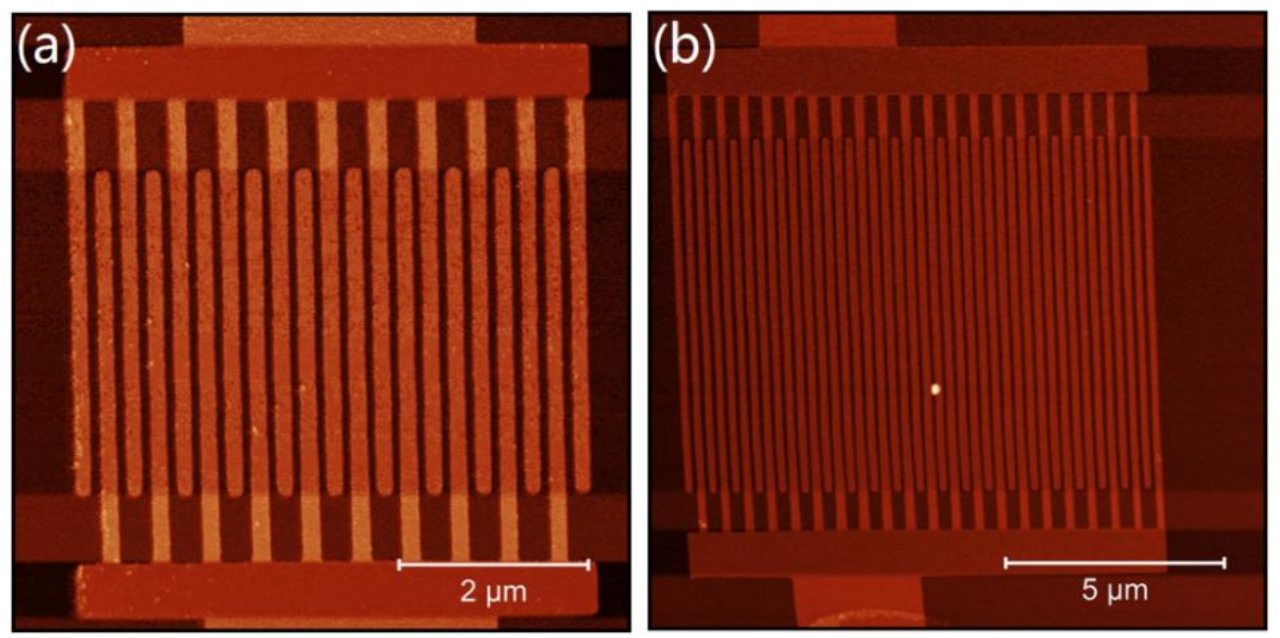

Figure 4.4: AFM images of $\mathrm{Au}$ interdigitated electrodes on $\mathrm{SiO}_{2}$ substrates. The gap between the $(3 \mathrm{~nm} \mathrm{Ti} / 15 \mathrm{~nm}$ $\mathrm{Au}$ ) electrodes is $100 \mathrm{~nm}$. (a) 20 gaps and $3.5 \mu \mathrm{m}$ Au strip length. (b) 41 gaps and $8 \mu \mathrm{m}$ Au strip length. 
To increase the conductance of the DXP devices, we adopted interdigitated Au electrodes with Ti adhesion layer (Fig. 4.4) on similar substrates, using the same fabrication techniques (EBL and lift-off) and cleaning methods.

\section{Light response}
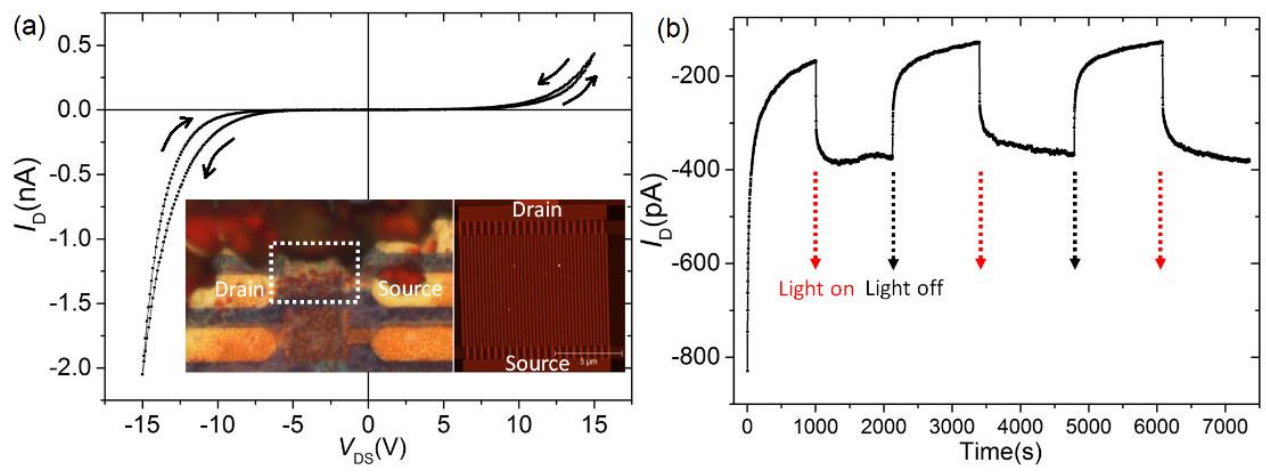

Figure 4.5: Light response of a DXP device. (a) $I V$ measurement recorded before the time-dependent measurement shown in (b). No gate voltage was applied. Arrows show the sweep direction of the voltage. Insets are an optical microscope image of the device after drop-casting DXP (left) and an AFM image of the electrode before dropcasting DXP (right), respectively. The white dashed box in the optical microscope image indicates the location of the measured device. The electrode has same parameters as the electrode of Fig. 4.4(b). (b) Light response during the time-dependent measurement $\left(V_{D S}=-10 \mathrm{~V}\right.$, no $\left.V_{G S}\right)$.

It has been reported that perylene diimide derivatives such as DXP could have applications in photosensitive devices in which the charge carrier concentration is modulated by exposure to light [15]. Therefore, we have checked the responsiveness of our DXP devices to light. After measuring the IV characteristics shown in Fig. 4.5(a), we kept the source-drain voltage at a constant value $(-10 \mathrm{~V})$ and then did a time-dependent measurement. The device was kept in the dark at the beginning of the measurement. During the time-dependent measurement, we intermittently exposed the sample to light generated by a halogen lamp. As expected, exposure to light boosts the current, which decreases again abruptly after blocking the light (Fig. 4.5(b)). This experiment shows that the observed conductivity is indeed related to DXP. It has been reported that the transient current in an organic semiconductor device can be explained by the charging and discharging processes of the photointroduced space charge, corresponding with the light-on and light-off regions respectively [16]. The space charge is usually ascribed to a poor mobility of the organic 
semiconductor [16] and can also relate to traps and defects in the device [17]. The latter is also discussed below.

\section{Degradation in air}

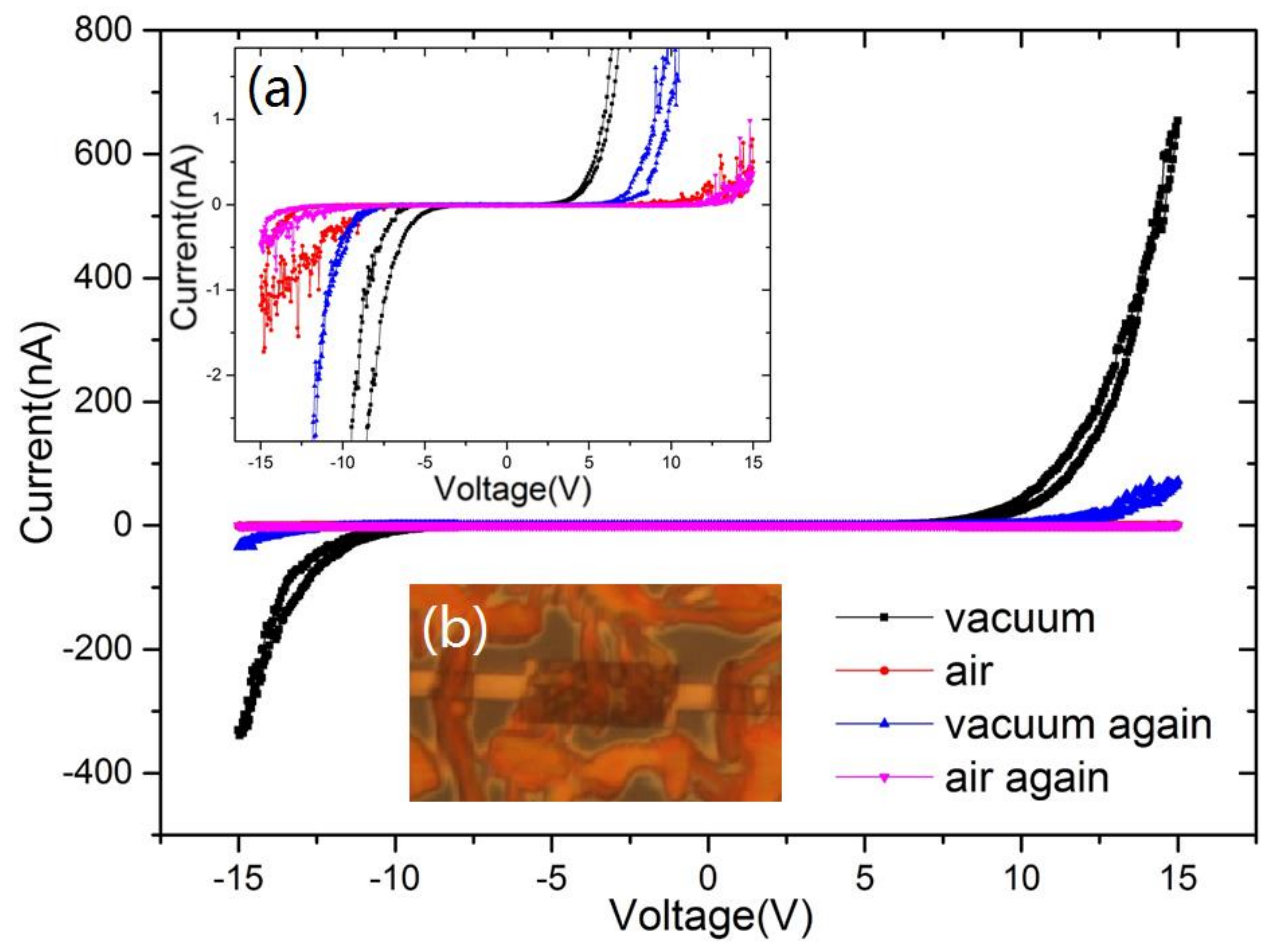

Figure 4.6: Current-voltage characteristics of a DXP device measured alternatively in high vacuum ( 10 $\left.0^{-5} \mathrm{mbar}\right)$ and air, respectively. Inset (a): zoom-in of the small current region. Inset (b): optical microscope image of the device.

Measurements in high vacuum is required, as the devices were found to be instable in the ambient or a rough vacuum generated by a roughing pump. Figure 4.6 shows the $I V$ results of a DXP device measured at RT in high vacuum ( $10^{-5} \mathrm{mbar}$ ) and in air, respectively. The inset (b) shows an optical microscope image of the device. We first measured in the vacuum, then in the air, back to the similar vacuum again afterwards, and went to the air again in the last measurement. The results clearly show that the device was much less conducting and less stable when measuring in the air than in the vacuum. Furthermore, once we measured the device in the air, the degradation is irreversible. Most probably, oxygen and/or water react with charged DXP molecules, as is already mentioned in Chapter 2. To suppress the 
effects of oxygen and water as much as possible, all measurements were carried out in high vacuum (unless specifically stated otherwise).

\section{Hysteresis}

The hysteresis observed in the IV characteristics (Fig. 4.5(a)) as well as the transient current observed in the time-dependent measurement (Fig. 4.5(b)) indicate that our DXP devices suffer from some degradation effects. Since we always measured in high vacuum, effects due to ambient gases should be suppressed. However, we cannot exclude the effect of defects and contamination at the $\mathrm{DXP} / \mathrm{SiO}_{2}$ interface, especially since no special treatment of the $\mathrm{SiO}_{2}$ surface was carried out, for example, HMDS treatment, before the deposition of DXP. It has been reported that the hysteresis can be related to such undesired properties of the organic/dielectric interface $[18,19]$. In addition, the DXP layer formed by drop-casting on the electrodes is disordered on the length scale of the devices. Consequently, structural defects such as grain boundaries could also introduce traps inside the organic layer and contribute to the hysteresis in the $I V$ characteristics [20,21]. To reveal whether such issues play a role in our devices, it is necessary to carry out further measurements of devices with DXP samples featuring different crystalline properties, such as evaporated and annealed DXP films, or single DXP crystals.

Lastly, the hysteresis might be attributed to "memristive" effects [22]. In memristive devices, ionic motion under large electric fields usually plays the main role. In addition, similar effects may occur when non-rigid structures are involved in nanoscale devices where large electric fields could be generated at even small voltages. Unless the memristive effect is controllable, repeatable and significant, so that memristive devices could be realized, it is undesired and should be suppressed by, for instance, making the nanostructures more rigid and/or avoiding large voltages.

\section{Output and transfer characteristics}

Electrical transport measurements of output (sweep source-drain voltage $V_{\mathrm{DS}}$, measure source-drain current $I_{D}$, and gate voltage $V_{G S}$ is fixed) and transfer (sweep $V_{G S}$, measure $I_{D}$ and $V_{D S}$ is fixed.) characteristics of DXP devices were performed. The devices had the same electrode layout as shown Fig. 4.4(b). Figures 4.7 and 4.8 show two typical results. The main difference between these two results is the maximum gate voltage we could apply. Because the $100 \mathrm{~nm} \mathrm{SiO} 2$ layer was not always equally insulating, most likely due to defect-related 
breakdown [23, 24], we could not apply equally high gate voltages for all devices. We kept $I_{\mathrm{G}}$ below $100 \mathrm{pA}$ when measuring the gate dependence to avoid breakdown.

Figures 4.7(b) and (c) show the output characteristics in the positive and negative sourcedrain voltage regions, respectively. The gate voltage bias was set at $-7.5 \mathrm{~V}$ and $+7.5 \mathrm{~V}$ alternatingly, and this alternation was repeated for three times. Each $I_{D}-V_{D S}$ curve is an average of four successive measurements (Fig. 4.7(a)). From these output characteristics we can see that the source-drain current is always larger for positive $V_{G S}$. Figures $4.7(e)$ and (f) show transfer characteristics recorded for positive and negative $V_{D S}$, respectively. $V_{G S}$ was swept between $-10 \mathrm{~V}$ and $10 \mathrm{~V}$ and $V_{D S}$, was kept at $0 \mathrm{~V}, \pm 2 \mathrm{~V}, \pm 4 \mathrm{~V}, \pm 6 \mathrm{~V}$ and $\pm 8 \mathrm{~V}$ respectively. Each curve is again an average of four successive measurements (Fig. 4.7(d)). The transfer characteristics show that source-drain current increases when the gate voltage is changed from negative to positive. The results of Fig. 4.8 show similar output and transfer characteristics. The above results indicate n-type behavior of the DXP devices. The unsaturated output characteristics are most probably due to the short channel effect (see Chapter 2). We can also see that the devices were not completely off when the gate voltages were zero. To verify that this behavior is indeed related to the short-channel effect, we need to systematically vary the channel length for the same gate dielectric thickness. Another issue evident in these measurements is the significant hysteresis in the transfer characteristics. As mentioned above, this is very likely due to bias stress effects (see also Chapter 2), involving undesired properties of the $\mathrm{DXP} / \mathrm{SiO}_{2}$ interface. In future work, we would need to improve the organic/dielectric interface, and address the effect of charge traps in the organic layer as well.

Even though the results discussed above are consistent with n-type conduction, it is difficult to rule out alternative explanations. For instance, it has been proposed that in short-channel devices, gate effects could be rooted in modulation of the injection barriers for electrons and holes. To suppress the influence of this effect, a low-work-function metal could be used for source and drain electrodes [25]. 

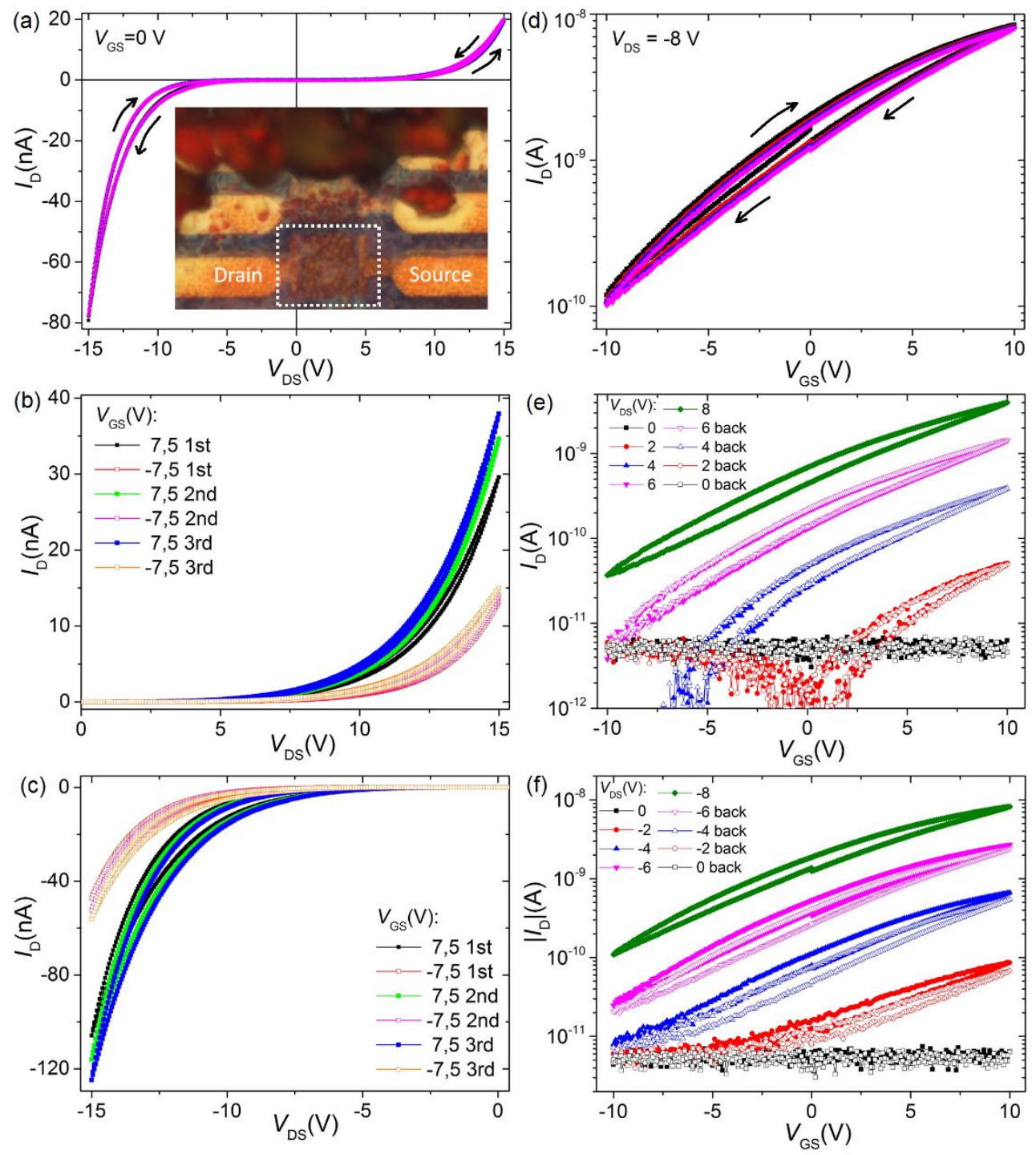

Figure 4.7: Output and transfer characteristics of a DXP device. (a) Four successive $I_{D}-V_{D S}$ measurements at $V_{G S}=$ $0 \mathrm{~V}$. Arrows show the sweep direction of $V_{D S}$. Inset: optical microscope image of the device (in the white dashed box). (b) Output characteristics in the positive $V_{D S}$ region. (c) Output characteristics in the negative $V_{D S}$ region. (d) Four successive $I_{D}-V_{G S}$ measurements at $V_{D S}=-8 \mathrm{~V}$. Arrows show the sweep direction of $V_{G S}$. (e) Transfer characteristics for the positive $V_{D S}$. (f) Transfer characteristics for the negative $V_{D S}$ 

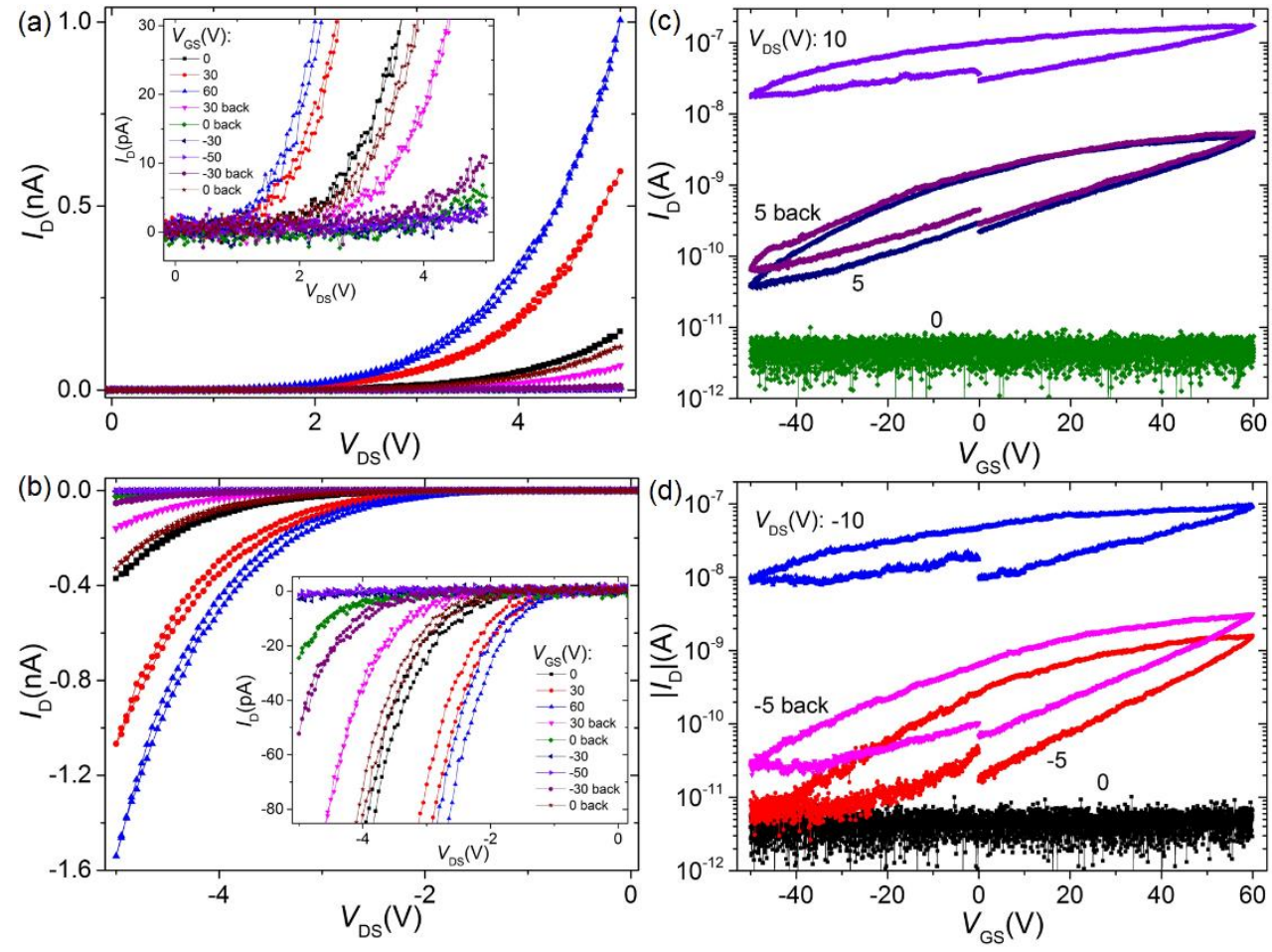

Figure 4.8: Output and transfer characteristics of a DXP device with better oxide quality. (a) Output characteristics in the positive $V_{D S}$ region. Inset is a zoom-in of the small $I_{D}$ region. (b) Output characteristics in the negative $V_{D S}$ region. Inset is a zoom-in of the small $I_{D}$ region. (c) Transfer characteristics for positive $V_{D S}$. (d) Transfer characteristics for negative $V_{D S}$. The sweep directions of $V_{D S}$ and $V_{G S}$ are the same as in Fig. 4.7. 


\subsection{Summary and outlook}

In summary, we measured the output and transfer characteristics of lateral DXP devices made by drop-casting DXP onto Au interdigitated electrodes. The electrical transport property of the devices were light sensitive. The devices were much more stable when measuring in vacuum ( $10^{-5} \mathrm{mbar}$ ) than in ambient atmosphere. Based on the output and transfer properties, the DXP devices exhibited n-type channel behavior.

Nevertheless, further experiments are necessary to confirm and improve the channel behavior and to make spin-dependent transport measurements possible. The first problem is the morphology of the DXP film. To achieve continuous, uniform films with a high degree of crystallinity, thermal evaporation and substrate heating during deposition are highly preferable. This might also suppress the hysteresis in both the output and transfer characteristics, if the hysteresis is related to charge trapping at structural defects in the DXP film. Secondly, passivation of the $\mathrm{SiO}_{2}$ surface is considered to be promising for decreasing the hysteresis in the transfer characteristics. Thirdly, to verify the short channel effect in the lateral DXP devices, we need to investigate systematically both long-channel and shortchannel devices. 


\section{References}

1. Kokil, A., K. Yang, and J. Kumar, Techniques for characterization of charge carrier mobility in organic semiconductors. Journal of Polymer Science Part B: Polymer Physics, 2012. 50(15): p. 1130-1144.

2. Boehme, C. and J.M. Lupton, Challenges for organic spintronics. nature nanotechnology, 2013. 8(9): p. 612-615.

3. Bobbert, P., Is there more than meets the eye? Nature nanotechnology, 2013. 8(12): p. 887-887.

4. McCamey, D., et al., Hyperfine-field-mediated spin beating in electrostatically bound charge carrier pairs. Physical review letters, 2010. 104(1): p. 017601.

5. Marumoto, K., et al., Spatial extent of wave functions of gate-induced hole carriers in pentacene field-effect devices as investigated by electron spin resonance. Physical review letters, 2006. 97(25): p. 256603.

6. Behrends, J., et al., Bipolaron formation in organic solar cells observed by pulsed electrically detected magnetic resonance. Physical review letters, 2010. 105(17): p. 176601.

7. Dehollain, J., et al., Nanoscale broadband transmission lines for spin qubit control. Nanotechnology, 2012. 24(1): p. 015202.

8. Wang, L., et al., Insight into crystallization process of rubrene by binary solvent mixtures. RSC Advances, 2016. 6(5): p. 3532-3538.

9. Kumatani, A., et al., Solution-processed, self-organized organic single crystal arrays with controlled crystal orientation. Scientific reports, 2012. 2: p. 393.

10. Ron, H., S. Matlis, and I. Rubinstein, Self-assembled monolayers on oxidized metals. 2. Gold surface oxidative pretreatment, monolayer properties, and depression formation. Langmuir, 1998. 14(5): p. 1116-1121.

11. Baumans, X.D., et al., Thermal and quantum depletion of superconductivity in narrow junctions created by controlled electromigration. Nature communications, 2016. 7.

12. Zandbergen, H.W., Electromigration. http://www.tnw.tudelft.nl/?id=36285\&L=1 (Date of access: 20-12-2016)

13. Kim, H., et al., Electromigration-induced failure of GaN multi-quantum well light emitting diode. Electronics Letters, 2000. 36(10): p. 1.

14. Geden, B., Understand and Avoid Electromigration (EM) \& IR-drop in Custom IP Blocks. Synopsys White Paper, 2011. 
15. Rekab, W., et al., High-Performance Phototransistors Based on PDIF-CN2 SolutionProcessed Single Fiber and Multifiber Assembly. ACS applied materials \& interfaces, 2016. 8(15): p. 9829-9838.

16. Hu, L., et al., Organic optoelectronic interfaces with anomalous transient photocurrent. Journal of Materials Chemistry C, 2015. 3(20): p. 5122-5135.

17. Dacuña, J. and A. Salleo, Modeling space-charge-limited currents in organic semiconductors: Extracting trap density and mobility. Physical Review B, 2011. 84(19): p. 195209.

18. Okamoto, H., et al., Transistor application of alkyl-substituted picene. Scientific reports, 2014. 4.

19. Singh, M., et al., Bio-sorbable, liquid electrolyte gated thin-film transistor based on a solution-processed zinc oxide layer. Faraday Discuss., 2014. 174: p. 383-398.

20. Sirringhaus, H., Reliability of organic field-effect transistors. Advanced Materials, 2009. 21(38-39): p. 3859-3873.

21. Ucurum, C., et al., Hole trap related hysteresis in pentacene field-effect transistors. Journal of Applied Physics, 2008. 104(8): p. 4501.

22. Strukov, D.B., et al., The missing memristor found. nature, 2008. 453(7191): p. 8083.

23. Wolters, D. and J. Vanderschoot, DIELECTRIC-BREAKDOWN IN MOS DEVICES. 1. DEFECT-RELATED AND INTRINSIC BREAKDOWN. Philips Journal of Research, 1985. 40(3): p. 115-136.

24. Dumin, D., Oxide reliability: a summary of silicon oxide wearout, breakdown, and reliability. Vol. 23. 2002: World Scientific.

25. Collet, J., et al., Low-voltage, $30 \mathrm{~nm}$ channel length, organic transistors with a selfassembled monolayer as gate insulating films. Applied Physics Letters, 2000. 76(14): p. 1941-1943. 


\section{Chapter 5}

\section{Charge transport in nanoscale vertical P3HT pillar devices ${ }^{1}$}

\subsection{Introduction and motivation}

For application in light-emitting diodes, field-effect transistors and solar cells, organic semiconductors (OSCs) are playing an increasingly important role, owing to their easy processability and suitability for low-cost and flexible electronics [1, 2]. Properties such as charge-carrier mobility, solution-processability, crystallinity and interface properties are important for implementation of organic semiconductors into electronic devices [3]. For many applications, like organic light-emitting transistors [4] or display pixel drivers [5], it is crucial to achieve high frequencies ( $10 \mathrm{MHz})$ and large current densities $\left(10-20 \mathrm{~mA} / \mathrm{cm}^{2}\right)$ [6] to improve the device performance. This can be realized by choosing organic semiconductors with high carrier mobility $[7,8]$, or by reducing the channel length down to the nanoscale [9], as demonstrated in this chapter.

For the fabrication of such nanoscale junction lengths in planar devices, source and drain electrodes have to be patterned by nanolithography techniques [10, 11]. A vertical configuration, where the OSC thin film is sandwiched between two (metallic) contacts, is very attractive because the channel length is defined by the thickness of the OSC layer, which is very well controllable down to a few $\mathrm{nm}$, whereas the device area is given by the overlap of the contacts $[12,13]$. Vertical geometries enable the investigation of charge transport in organic semiconductors at the nanoscale. They are already commonly used in molecular monolayer junctions $[14,15]$, and present several advantages in comparison to planar structures. At small junction thickness, the electric field at low voltage can still be very high. The junctions can thus operate at low voltages, while maintaining sizeable current densities, which is beneficial for implementation in low-power electronic devices like organic light-emitting diodes and organic field-effect transistors [4, 16].

Top contacting and nanopatterning of thin layers of OSC are not straightforward. Direct metal evaporation can result in penetration through the organic film, often leading to damage of the film and electrical shorts $[17,18]$. Standard lithography methods that

\footnotetext{
${ }^{1}$ This chapter is based on Wilbers, J.G.E., et al., Charge transport in nanoscale vertical organic semiconductor pillar devices. Sci. Rep. 7, 41171; doi: 10.1038/srep41171 (2017).
} 
generally include optical or electron-beam lithography resists and developers, as well as liftoff procedures in solvents like acetone or dimethyl sulfoxide (DMSO), cannot be applied to pattern OSC, because most of them are affected by these chemicals [19, 20]. There are methods to avoid damage of the organic layer, for example, by indirect evaporation of metals onto cooled samples through a shadow mask [21] or buffer-layer-assisted deposition [22]. However, those techniques do not allow lateral nanostructuring. Other approaches for top contacting organic thin films and/or monolayers are so-called "soft-landing" techniques such as transfer printing [23, 24], the use of conductive polymers [25] or liquid metals [26] as top contacts, lift-off float-on (LOFO) [27] and polymer-assisted lift-off (PALO) [28]. These methods, which are mainly utilized for the fabrication of molecular tunnel junctions, all have their own advantages and disadvantages. They either introduce an additional resistance or an oxide layer, or hazardous chemicals are used during processing. In Ref. [29] a photoresist compatible with OSC was used, enabling direct patterning of the organic layers. However, in that study, the metal contact is directly evaporated onto the organic thin film, which can, as already mentioned, lead to electrical shorts, especially for thin organic films.

In this chapter, we demonstrate a method to realize vertical organic devices with ultrashort junction lengths down to $5 \mathrm{~nm}$. Thin layers of the $p$-type organic semiconductor regioregular poly(3-hexylthiophene) (Р3HT) were gently contacted by wedging transfer [30], and subsequently further structured by dry etching. By applying this water-based technique [30], EBL-patterned metal electrodes ( $200 \mathrm{~nm}$ to $2 \mu \mathrm{m}$ in diameter $d$ ) are gently transferred onto $\mathrm{P} 3 \mathrm{HT}$. This is the only step in which EBL is applied. All the other steps are either self-aligning or achieved with standard photolithography. In earlier work, we showed that we were able to contact self-assembled monolayers (SAMs) of alkanethiols of different lengths by wedging transfer [31]. We now optimized this technique for contacting and patterning nanoscale P3HT devices. P3HT was used because it forms a smooth thin film that can be easily varied in thickness by changing the concentration and/or the spin-coating speed. We note that our water-based wedging-transfer technique could induce electron traps in the P3HT [32]. However, such traps are not expected to influence hole transport, which is the focus of our study. If necessary, water could be removed by (vacuum) annealing.

Regioregular P3HT is widely used for organic-based electronic devices due to its relatively high mobility as compared to other organic semiconductors [33-35], owing to crystallites that form via $\pi-\pi$ inter-chain self-assembly [34]. However, the mobility does not only depend on regioregularity and molecular weight, but also on the solvent used. The higher the boiling point of the solvent, the slower the solvent evaporation during and after spincoating, and the better the crystallinity of the thin film. A higher crystallinity gives better conductivities [35, 36]. For this reason and the high solubility for P3HT we used 
bromobenzene as solvent [36]. The ionization potential of P3HT strongly depends on the average chain length, chain torsion and packing density of the chains, which change the delocalization length and screening of charges [37]. The injection barrier for charge carriers depends on the position of the ionization potential with respect to the work function of the electrode material. Au was used as electrode material, because a low injection barrier is expected for Au-P3HT interfaces $[38,39]$. In order to achieve good current injection from the electrode into the organic semiconductor with low contact resistance, the work function has to be aligned to either the highest occupied molecular orbital (HOMO) energy or the lowest unoccupied molecular orbital (LUMO) energy of the organic film [40].

\subsection{Device fabrication}

In the following, device fabrication is briefly described. More details are elaborated in Ref. [41]. The complete fabrication process is shown schematically in Fig. 5.1. P3HT was spincoated onto clean Au bottom electrodes on $\mathrm{Si} / \mathrm{SiO}_{2}$ substrates (Figs. 5.1(a) and (b)). The thickness was varied by different $\mathrm{P} 3 \mathrm{HT}$ concentrations in the solvent and spin-coating speeds. In this chapter, the P3HT layer thickness $t=5,10,40$ and $100 \mathrm{~nm}$. For wedging transfer hydrophilic surfaces are required. For the previous SAM devices [31], the molecules were only present on the metal bottom electrodes, while the rest of the substrate remained hydrophilic. This is different for the P3HT devices. P3HT was spin-coated over the whole substrate, leading to a completely hydrophobic surface. Therefore, we first removed P3HT from the unpatterned upper part of the $\mathrm{SiO}_{2}$ substrate, and subsequently wedging transfer was performed (not shown in the figure) [30,31]. The metal top contacts (70 nm thick Au disks with a diameter $d$ between $200 \mathrm{~nm}$ and $2 \mu \mathrm{m}$ ) were wedge-transferred onto the P3HT. For wedging transfer the top contacts were embedded in a hydrophobic polymer, cellulose acetate butyrate $(C A B)$. The cellulose polymer was prepared by dissolving the cellulose acetate butyrate $(C A B)$ in ethyl acetate at a concentration of $30 \mathrm{mg} / \mathrm{ml}$ under stirring for 30 min. The important property of the cellulose polymer is its hydrophobicity. When the device is dipped onto water, the water penetrates in between the hydrophilic $\mathrm{SiO}_{2}$ substrate and the hydrophobic $C A B$ and thereby the polymer is lifted off. The Au disks are lifted off with the $\mathrm{CAB}$ polymer due to the low adhesion between $\mathrm{Au}$ and $\mathrm{SiO}_{2}$. The $\mathrm{Au}$ disks were wedgetransferred onto the new substrate with the $5 \mu \mathrm{m}$ wide bottom electrodes coated with $\mathrm{P} 3 \mathrm{HT}$ by placing the substrate under an angle of $\sim 45^{\circ}$ on a grid holder in a beaker with Milli$Q$ water and slowly pumping out the water (Fig. 5.1(c)). In this way, the cellulose polymer first makes contact with the hydrophilic $\mathrm{SiO}_{2}$ and then softly lands on the $\mathrm{P} 3 \mathrm{HT}$. In the next step, directional reactive ion etching (RIE) was performed to form vertical pillars (Fig. 5.1(d)). The Au disks served as an etch mask for the P3HT. Next, the pillars were embedded in an 
electrically insulating layer of hydrogen silsesquioxane (HSQ), which is thinner on top of the pillars than on the substrate (Fig. 5.1(e)). The Au top contacts of the pillars were exposed by RIE, while the organic layer was still protected (Fig. 5.1(f)) [42]. Subsequently, in order to contact the pillar structures, a $100 \mathrm{~nm}$ thick metal layer was deposited and patterned by photolithography to form large contact pads (Fig. 5.1(g)).

Scanning electron microscopy (SEM) was used to image a cross-section of a metal-P3HTmetal test device after directional dry etching and spin coating of HSQ (Fig. 5.2). The top Au served as an etch mask for the underlying P3HT thin film (Fig. 5.2(a)). In Figs. 5.2(c), a final test structure with large top contacts is shown.

(a)

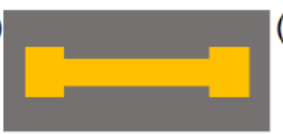

bottom electrodes

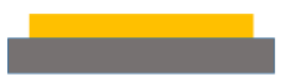

(e)

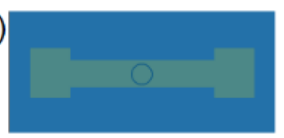

HSQ planarization

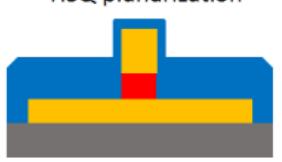

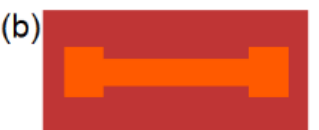

P3HT spin-coating

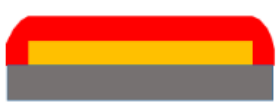

(f)

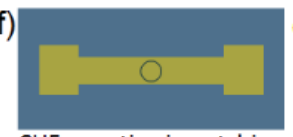

$\mathrm{CHF}_{3}$ reactive ion etching $\downarrow \downarrow \downarrow \downarrow \downarrow \downarrow \downarrow \downarrow \downarrow \downarrow \downarrow \downarrow \downarrow$

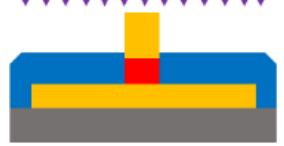

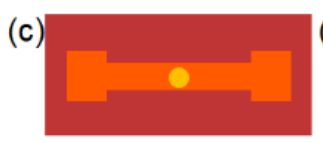

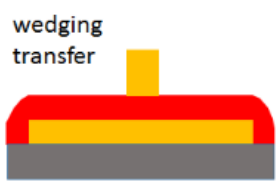

(g)

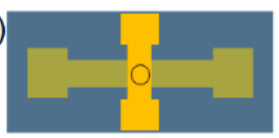

large top electrodes

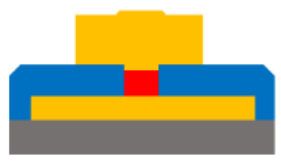

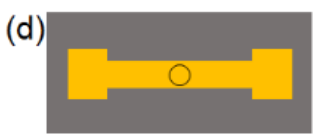
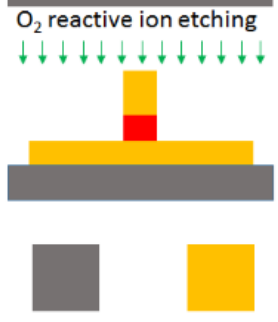

$\mathrm{SiO}_{2} \quad \mathrm{Au}$
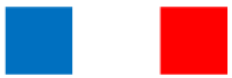

HSQ P3HT

Figure 5.1: Fabrication steps for vertical Au-P3HT-Au pillars. In each subfigure, the top is the top view and the bottom is the side view of the devices. (a) Fabrication of the bottom electrodes ( $\mathrm{Au}$, with Ti adhesion layer) on the $\mathrm{SiO}_{2}$ substrates by photolithography. (b) Spin-coating the P3HT. (c) Wedging transfer of the EBL-patterned Au disks. (d) RIE with $\mathrm{O}_{2}$ ions for pillar formation. (e) HSQ spin-coating; (f) $\mathrm{CHF}_{3}$ RIE to uncover the Au top contacts. (g) Evaporation of large top contacts patterned by photolithography. 

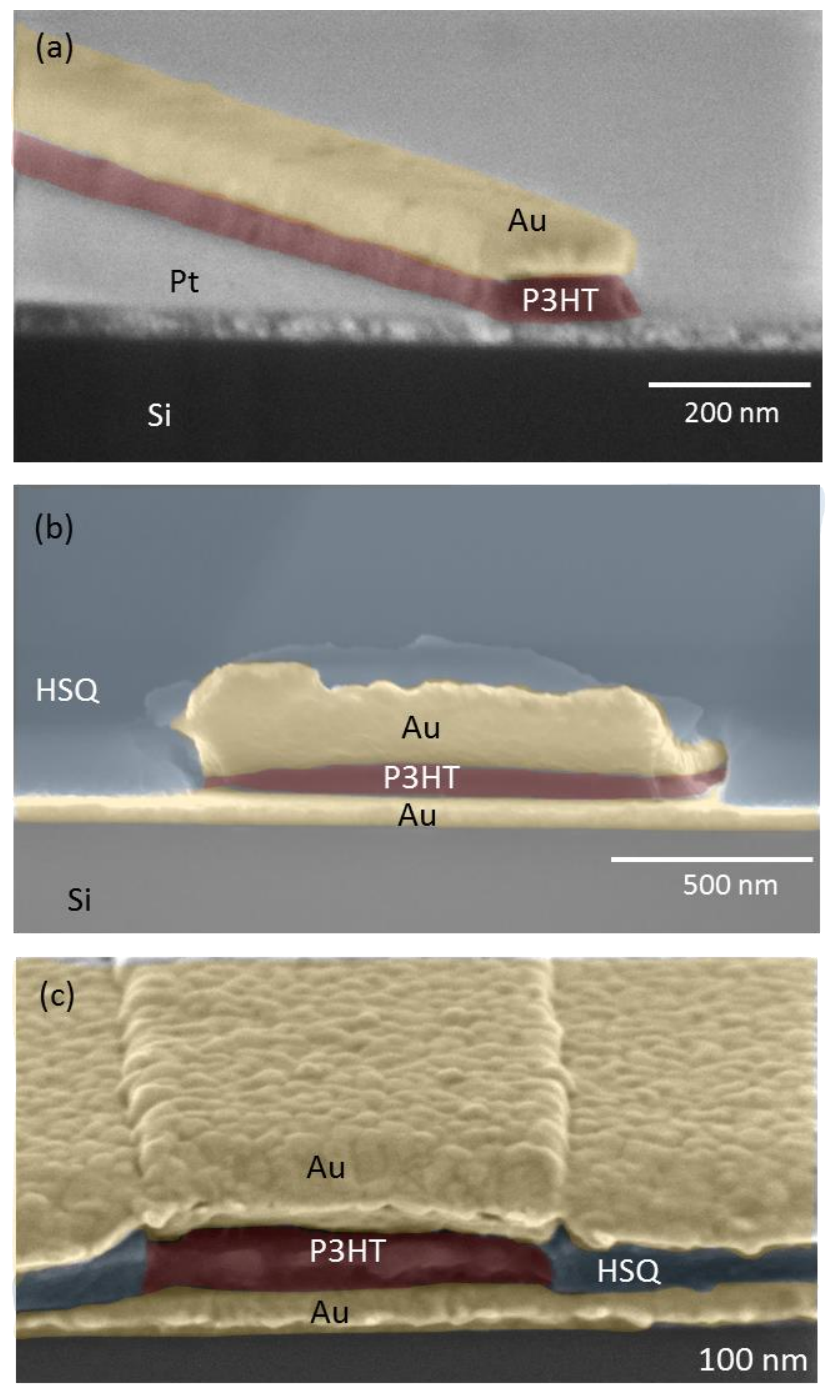

Figure 5.2: Colored SEM images of the cross-sections of the test devices. (a) Au-P3HT-Pt test structures (200 nm wide line). (b) Au-P3HT-Au structures ( $1 \mu \mathrm{m}$ wide line) embedded in HSQ. (c) Final Au-P3HT-Au structures (200 nm wide line) with large top contacts. 


\subsection{Electrical transport measurements}

We measured electrical transport properties of the two-terminal P3HT pillar devices at room temperature (RT, 294 or $295 \mathrm{~K}$ ) and the temperature dependence between RT and $150 \mathrm{~K}$ with steps of $50 \mathrm{~K}$. For the devices with $t=5,10$ and $40 \mathrm{~nm}$ P3HT thicknesses, the room temperature measurements were performed in a probe station (Janis ST-500) connected with custom built low noise electronics measurement instruments (IVVI-DAC rack, Quantum Transport designed instrumentation, designed by Ing. Raymond Schouten from Delft University of Technology [43]) which were controlled by a LabVIEW programme. The temperature dependence measurements were performed in a compressor-cooled Oxford Instrument with the low noise electronics measurement instruments. As for the devices with $t=100 \mathrm{~nm}$, we used Keithley 2400 as the voltage source because it has negligible output resistance compared to the device resistances. Similar to the air-instability of the DXP devices mentioned in Chapter 4, we found that the P3HT devices were not stable neither in air. Hence, the room temperature measurements were performed in the turbopumped vacuum ( $<10^{-4} \mathrm{mbar}$ ). As for the temperature dependence measurements, before introducing exchange gas (Helium) into the chamber of the Oxford Instrument, we pumped the chamber to a similar vacuum using a turbo pump.

Table 5.1 shows an overview of all (218) measured Au-P3HT-Au devices. There were 129 working devices and 89 non-working devices. A device is considered as "working" when it shows a non-linear IV characteristic that is stable over two consecutive sweeps within a factor of 2. The non-working devices have three different non-working styles, nonconductive, shorted, and unstable. A non-conductive device has current values in the noise level of the measurement equipment (1 pA). A shorted device shows a linear IV characteristic with small resistance of about hundreds of Ohms or a smaller value. An unstable device shows very large (larger than a factor of 2) fluctuations in the current signal during the $I V$ measurements. The instability of the unstable devices was probably due to filament/ion conducting behavior because of large electrical fields. The statistics in the table show that we achieved throughput of about 40-85\%, depending on the P3HT thicknesses. The relatively high working device yield indicates that the fabrication method is reliable, as even very thin layers in the sub-10 nm regime were successfully contacted and measured. 
Table 5.1: Overview of the yield of the Au-P3HT-Au devices for different P3HT thicknesses and pillar diameters. n.c. stands for non-conductive, us for unstable and s for shorted devices. * means that one device was not taken into account in Figs. 5.4 and 5.5 because current values of that device at larger biases reached the compliance of the measurement set-up.

\begin{tabular}{|c|c|c|c|c|}
\hline $\begin{array}{l}\text { P3HT thickness } \\
\text { [nm] }\end{array}$ & $\begin{array}{l}\text { Pillar diameter } \\
{[\mu \mathrm{m}]}\end{array}$ & $\begin{array}{l}\text { Total number of } \\
\text { junctions }\end{array}$ & $\begin{array}{l}\text { working } \\
\text { junctions }\end{array}$ & $\begin{array}{l}\text { non-working } \\
\text { junctions }\end{array}$ \\
\hline \multirow[t]{6}{*}{5} & 2 & 10 & $2\left(1^{*}\right)$ & 7 (s), 1 (us) \\
\hline & 1 & 11 & 6 & $\begin{array}{c}3 \text { (s), } 1 \text { (n.c.) } 1 \\
\text { (us) }\end{array}$ \\
\hline & 0.5 & 16 & 8 & 6 (s), 2 (n.c.) \\
\hline & 0.4 & 1 & 1 & \\
\hline & 0.3 & 15 & 4 & 10 (s), 1 (n.c.) \\
\hline & 0.2 & 1 & 1 & \\
\hline \multirow[t]{4}{*}{10} & 2 & 29 & 19 & 8 (s), 2 (n.c.) \\
\hline & 1 & 35 & 22 & 10 (s), 3 (n.c.) \\
\hline & 0.5 & 39 & 26 & 1, (s), 12 (n.c.) \\
\hline & 0.2 & 12 & & $\begin{array}{c}10 \text { (n.c.), } 2 \\
\text { unstable }\end{array}$ \\
\hline \multirow[t]{4}{*}{40} & 2 & 8 & 7 & $1(\mathrm{~s})$ \\
\hline & 1 & 10 & 9 & 1 (n.c.) \\
\hline & 0.5 & 9 & 7 & 1 (s), 1 (n.c.) \\
\hline & 0.2 & 9 & 6 & 3 (n.c.) \\
\hline \multirow[t]{2}{*}{100} & 2 & 4 & 4 & \\
\hline & 1 & 9 & 7 & 2 (n.c.) \\
\hline total & & 218 & 129 & 89 \\
\hline
\end{tabular}

We checked the stability in the vacuum of some working devices by performing consecutive $I V$ measurements at RT in short time span (e.g., in few hours or measured overnight) and IV measurements in every few days, without breaking the vacuum during the whole stability measurements. Figure 5.3 shows typical stability measurement results. From the results we can see that the devices were stable during the consecutive $I V$ measurements in the short time span (Figs. 5.3(a) and 5.3(c)). The variations between measurements over days are also very small (Figs. 5.3(b) and 5.3(d)). IV measurements were always performed by backward and forward voltage sweeps and no significant hysteresis was observed. We verified that the P3HT devices were also stable in the helium environment with the high background vacuum by performing similar $I V$ measurements. 
(a)
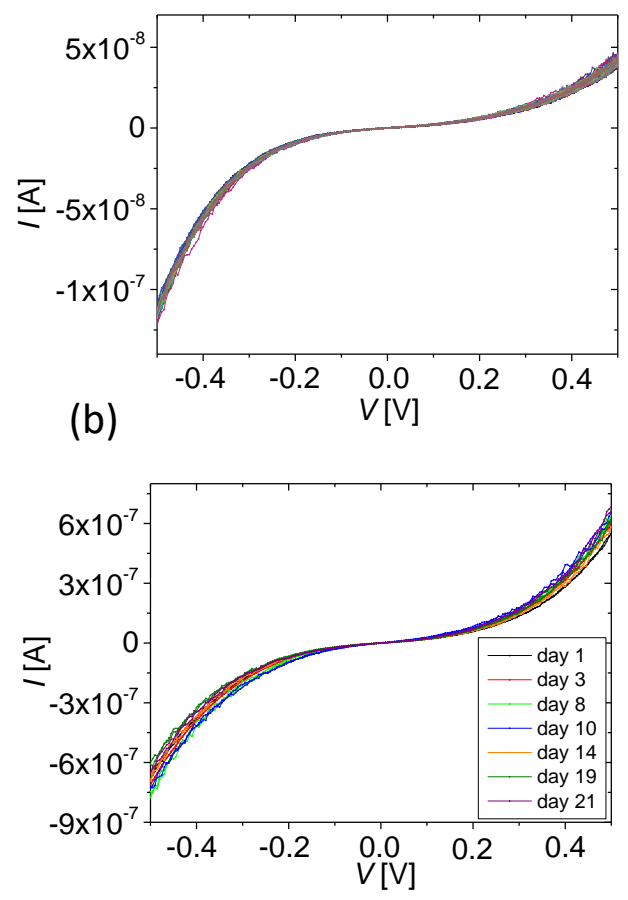

(c)
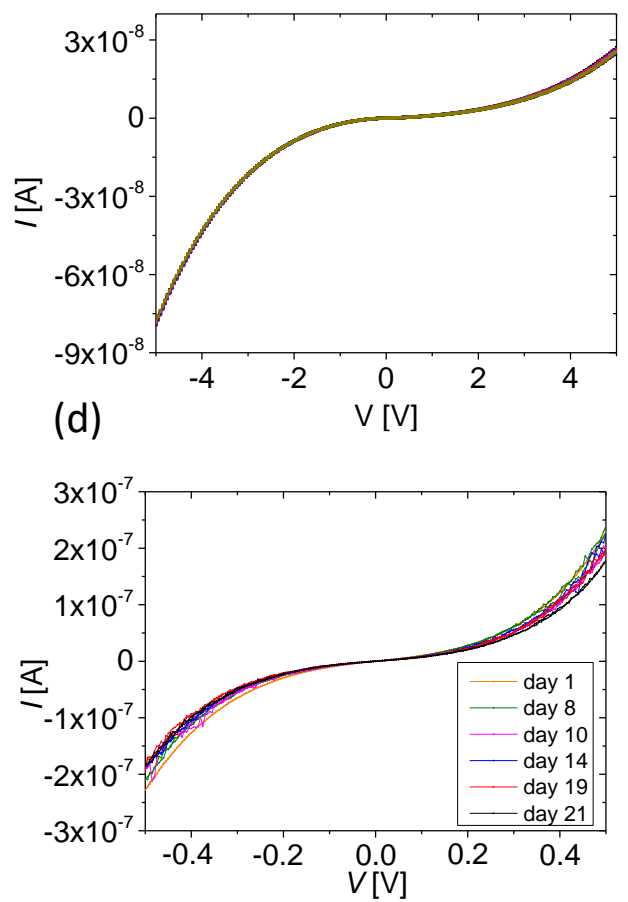

Figure 5.3: Vacuum-stability of the Au-P3HT-Au devices measured at RT in vacuum ( $10^{-4}$ mbar). (a) 40 consecutive $I V$ sweeps of a device with $t=10 \mathrm{~nm}$ P3HT thickness and $d=1 \mu \mathrm{m}$ P3HT diameter. (b) $I V$ measurements over days of a device with $t=5 \mathrm{~nm}$ and $d=500 \mathrm{~nm}$. (c) 10 consecutive $I V$ sweeps of a device $t=100 \mathrm{~nm}$ and $d=2$ $\mu \mathrm{m}$. (d) $I V$ measurements over days of a device with $t=5 \mathrm{~nm}$ and $d=1 \mu \mathrm{m}$.

Figures 5.4-5.6 show the RT measurement results of the P3HT devices. In Fig. 5.4, we firstly took 10 -base logarithmic value of the current densities which were calculated by dividing the measured current data by the nominal junction areas of the devices. Then we averaged the logarithmic value of $J$ over all junction diameters for each P3HT thickness, and plotted as a function of voltage. The error bars represent the standard deviation of the log $J$ values and provide an indication of the spread in J for devices with the same P3HT thickness. This is a common way of plotting data for molecular junctions [26, 44-46]. Hereby a normal distribution of $J$ is assumed, because $J$ is exponentially dependent on the thickness of the organic film, which can vary due to electrode surface defects and also roughness of the organic layer itself [47]. This approach is also appropriate for our devices with P3HT thicknesses of $5 \mathrm{~nm}$ and $10 \mathrm{~nm}$. In order to treat all devices equally, we also applied this approach for $40 \mathrm{~nm}$ and $100 \mathrm{~nm}$ thick P3HT. During the $I V$ measurements, we used larger voltage increments for the devices with thicker $\mathrm{P} 3 \mathrm{HT}$ to decrease measurement time. This 
is why Fig. 5.4 shows different numbers of data points in the $J-V$ characteristics of the devices with different thicknesses. The highly reproducible current densities are on the order of $10^{2}$ up to $10^{6} \mathrm{~A} / \mathrm{m}^{2}$, depending on the $\mathrm{P} 3 \mathrm{HT}$ thickness. Our devices with the thinnest ( $t=10 \mathrm{~nm}$ and $5 \mathrm{~nm}$ ) P3HT layers carry exceptionally high current densities, to our knowledge not reported for $\mathrm{P} 3 \mathrm{HT}$ devices so far. The devices with $t=100 \mathrm{~nm}$ show current densities of about $10^{2} \mathrm{~A} / \mathrm{m}^{2}$ at $0.5 \mathrm{~V}$, which is comparable to other P3HT-based devices sandwiched between indium tin oxide bottom electrodes and evaporated Au top contacts with similar P3HT thickness in Ref. [48].

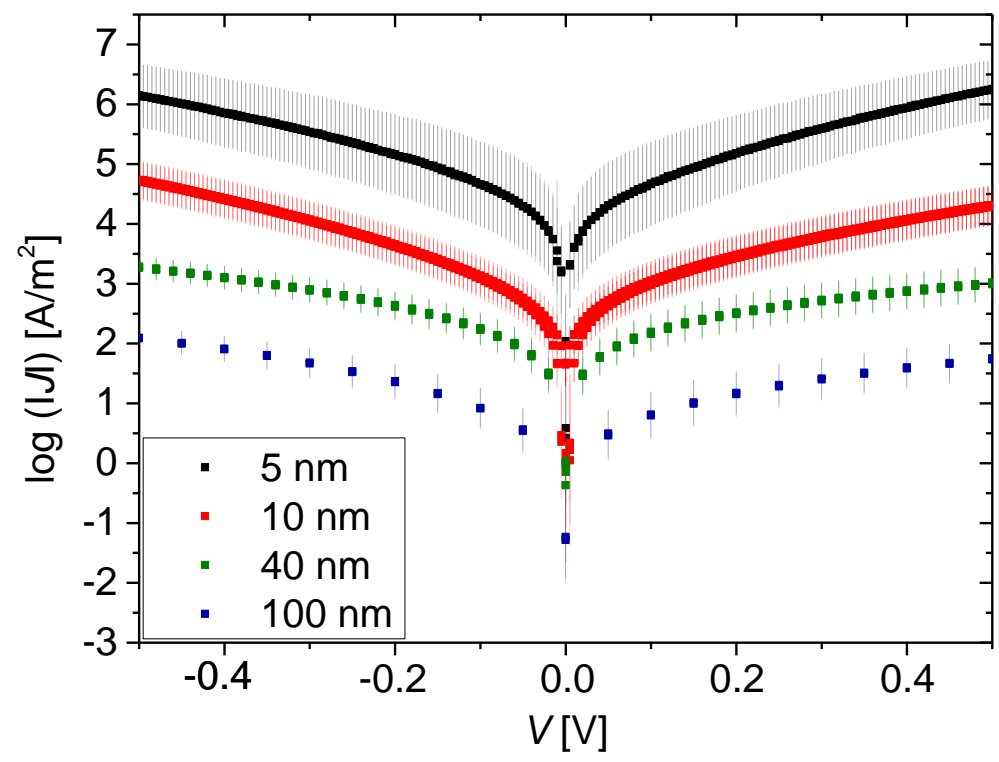

Figure 5.4: Electrical characterization of Au-P3HT-Au devices at RT in vacuum $\left(<10^{-4} \mathrm{mbar}\right)$ between $\pm 0.5 \mathrm{~V}$. The $J-V$ characteristics were obtained by taking the logarithmic average of the current densities of the devices with the same thicknesses. Black: $t=5 \mathrm{~nm}$ P3HT (steps: $5 \mathrm{mV}$ ), red: $t=10 \mathrm{~nm}$ P3HT (steps: $5 \mathrm{mV}$ ), green: $t=40 \mathrm{~nm} \mathrm{P3HT}$ (steps: $20 \mathrm{mV}$ ), $t=100 \mathrm{~nm}$ P3HT (steps: $50 \mathrm{mV}$ ).

We also plotted the $J-V$ characteristics of the devices with different P3HT diameters for each P3HT thickness in the left of Figs. 5.5 and 5.6. The right of Figs. 5.5 and 5.6 show the corresponding distribution of $\log (|J|)$ at certain voltages. Figures $5.5(\mathrm{~b})$ and 5.6 demonstrate that the current densities for $\mathrm{P} 3 \mathrm{HT}$ thicknesses $t=10,40$, and $100 \mathrm{~nm}$ slightly varied with respect to the junction diameter showing the trend of higher $J$ values for larger $d$. This is probably because the edge of the P3HT pillars was affected during the RIE step. And this affect has stronger impact on the devices with smaller P3HT diameters since the ratio of affected to non-affected area is larger for the devices with smaller diameters than 
the devices with larger diameters. However, in Fig. 5.5(a), there is no such trend. This is probably due to a shorter etching time for $5 \mathrm{~nm}$ thick P3HT. Hence, the affection from the RIE is not that strong, compared to the devices with thicker P3HT.

(a)

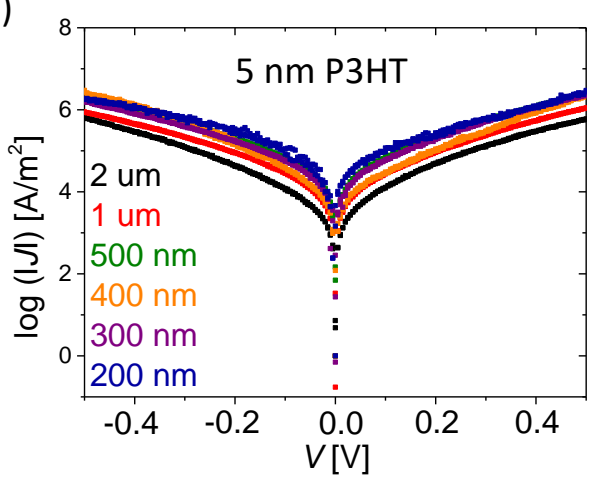

(b)

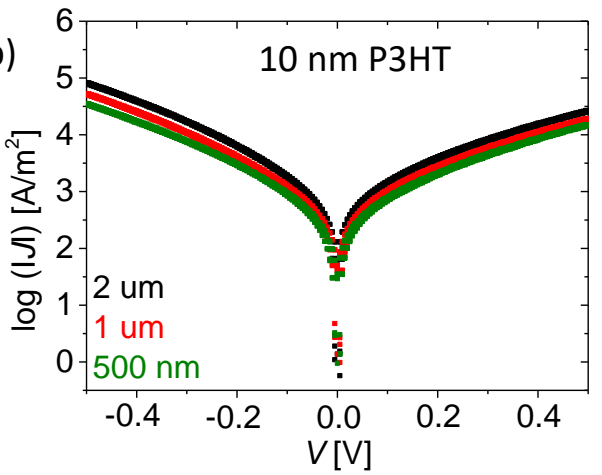

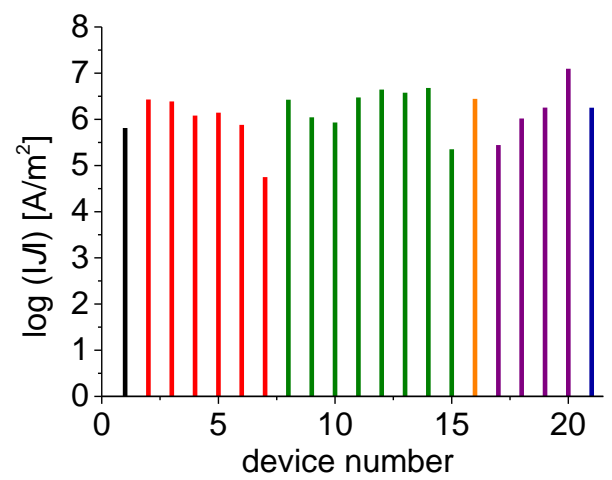

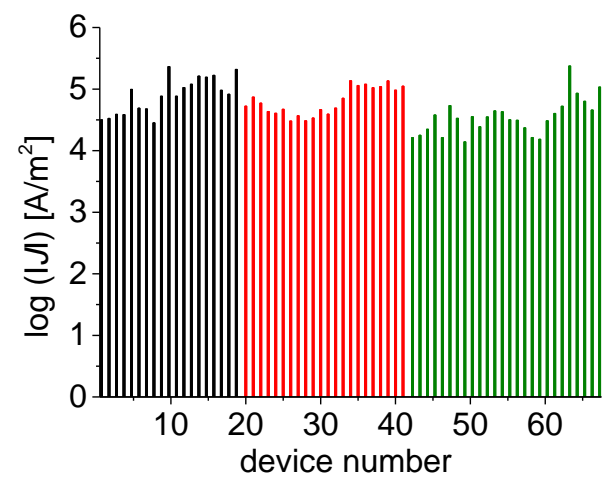

Figure 5.5: Electrical characterization of Au-P3HT-Au devices at RT in vacuum $\left(<10^{-4} \mathrm{mbar}\right)$. In the left figures, the current densities were obtained by taking the logarithmic average of the current densities for each junction diameter. The right figures show the corresponding distribution of $\log (|J|)$ at $-0.5 \mathrm{~V}$. The different colors stand for the junction diameter: black: $2 \mu \mathrm{m}$, red: $1 \mu \mathrm{m}$, green: $500 \mathrm{~nm}$, orange: $400 \mathrm{~nm}$, purple: $300 \mathrm{~nm}$, blue: $200 \mathrm{~nm}$. The P3HT thickness is (a) $5 \mathrm{~nm}$ and (b) $10 \mathrm{~nm}$.

Although top and bottom contacts are made from the same metal (Au), we observe a slightly asymmetric $J-V$ behavior (Figs. 5.3-5.6), which we attribute to differences in the top and bottom Au-P3HT interfaces. P3HT was spin-coated onto UV/ozone-cleaned bottom electrodes and subsequently annealed, while the top electrodes were applied by wedging transfer onto the $\mathrm{P} 3 \mathrm{HT}$ which was exposed to air and water. The energy level alignment between the Au work function and the P3HT HOMO is influenced by the interface, and can thus change during processing [38]. Also, the local packing of the P3HT chains at either interface is likely to be different. It is known that there is a strong dependence of the charge 
transport $[49,50]$ and energy level alignment [51] on the local packing. P3HT thin films contain crystalline areas, formed by $\pi-\pi$ interchain stacking, that are surrounded by an amorphous matrix [34]. The structure of the first nanometers is expected to be different from the bulk of the film [52]. This plays especially a role in vertical devices, while it is less critical for planar structures, where, e.g., in a field-effect transistor, the current flows in a thin region above the dielectric between the source and drain contacts.

(a)
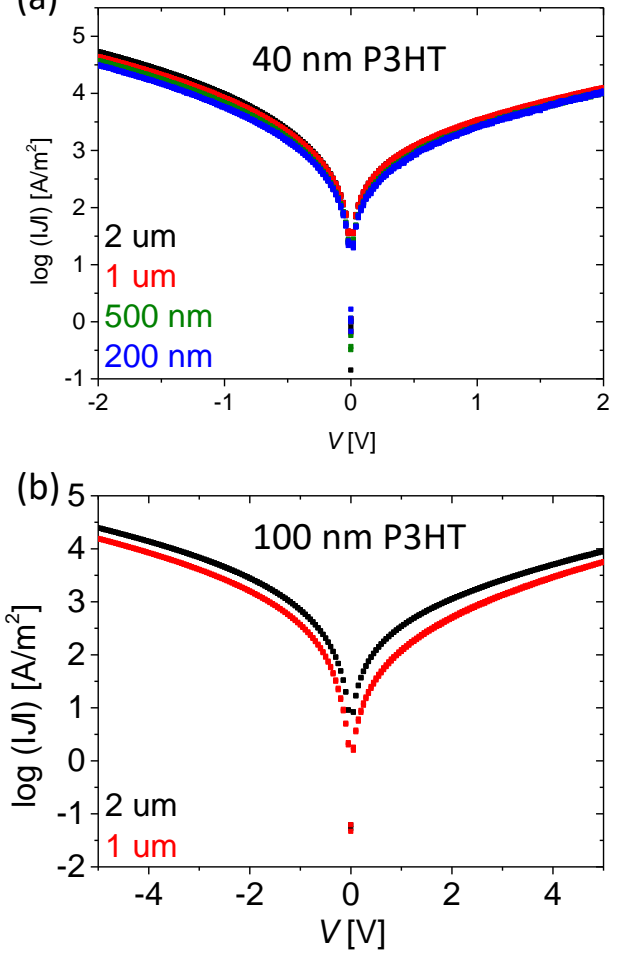
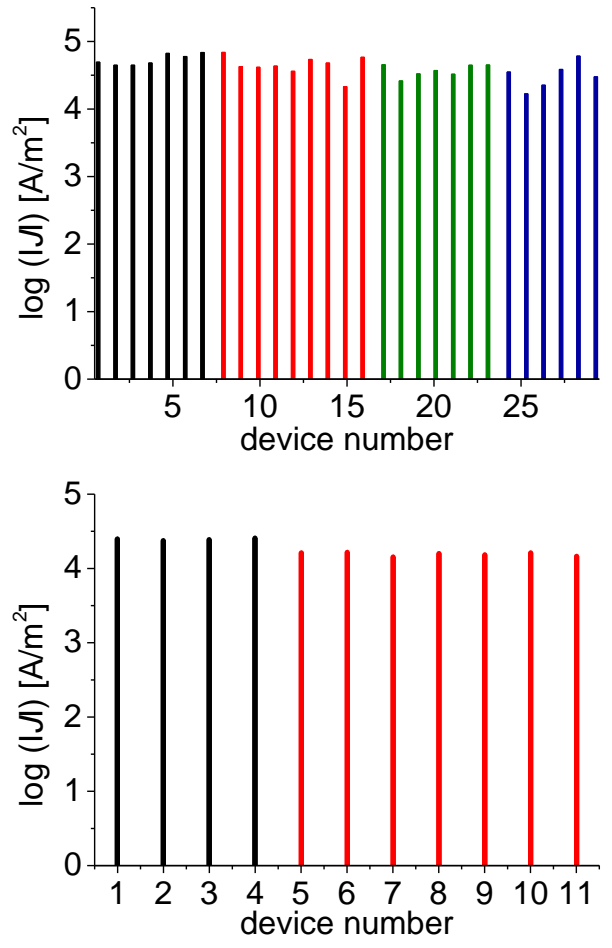

Figure 5.6: Electrical characterization of Au-P3HT-Au devices at RT in vacuum $\left(<10^{-4} \mathrm{mbar}\right)$. In the left figures, the current densities were obtained by taking the logarithmic average of the current densities for each junction diameter. The right figures show the corresponding distribution of $\log (|J|)$ at (a) $-0.5 \vee$ and (b) $-5 \vee$. The different colors stand for the junction diameter: black: $2 \mu \mathrm{m}$, red: $1 \mu \mathrm{m}$, green: $500 \mathrm{~nm}$, blue: $200 \mathrm{~nm}$. The P3HT thickness is (a) $40 \mathrm{~nm}$ and (b) $100 \mathrm{~nm}$.

The temperature dependence measurement results are shown in Figs. 5.7 and 5.8. The left of the figures shows the Arrhenius plots of the devices for each P3HT thickness at certain biases, and the right shows the distribution of $\log (|J|)$ at certain biases. From the results we can see that the conductivity of the devices dropped when the temperature decreased. The bar diagrams in the right reveal that there is almost no difference between the results 
measured in the cooling-down and the warming-up cycles, which indicates that the devices exhibited good stability in the cooling-down and warming-up cycle. As introduced in Chapter 2, electrical transport properties in disordered organic materials is usually understood as the hopping transport mechanism [53, 54], which is also called thermal(phonon)-assisted tunneling mechanism. The measured results also indicate thermal-assisted transport characteristic. To better understand the temperature dependence measurement results, we executed numerical modeling and the results are discussed below.

(a)
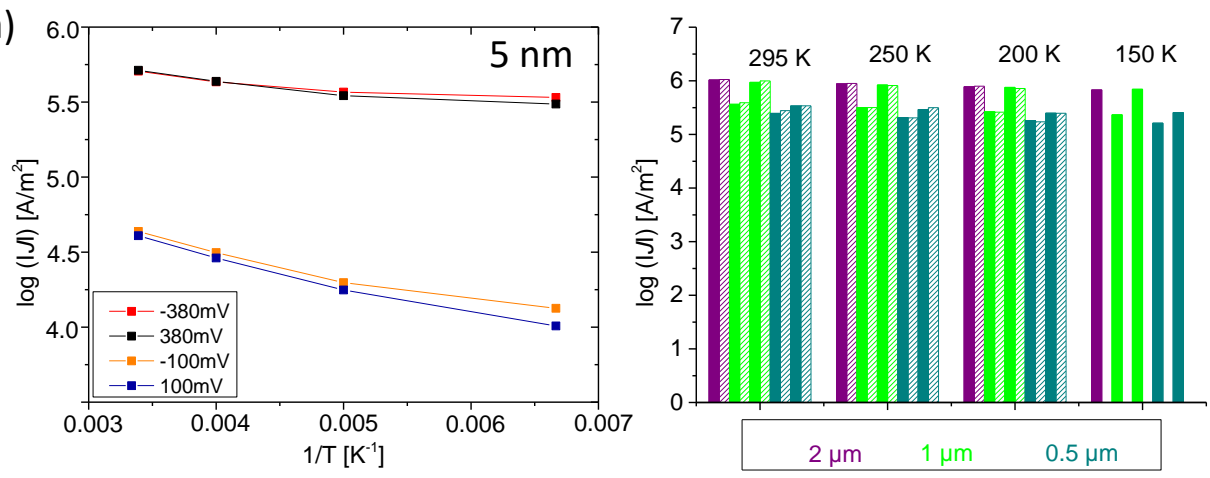

(b)
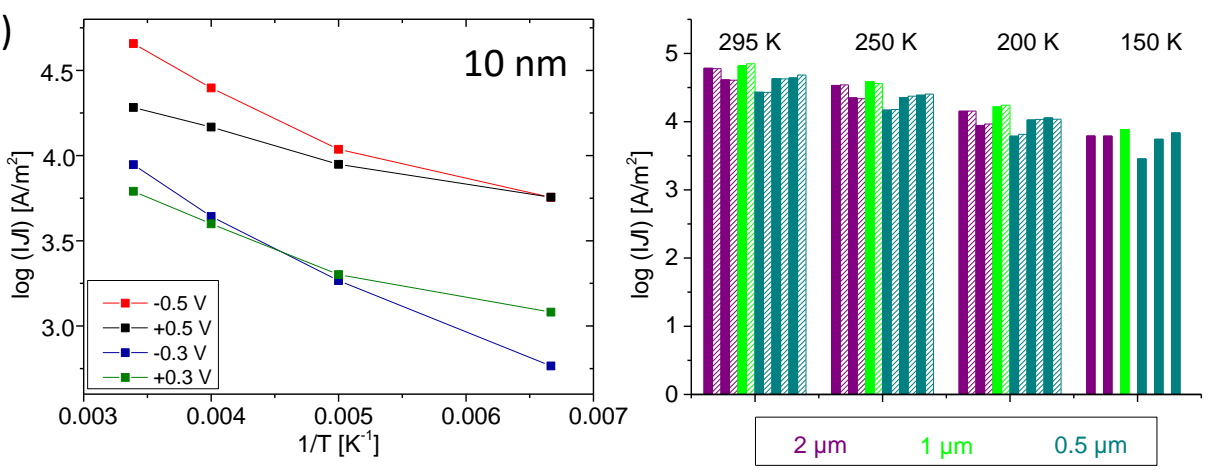

Figure 5.7: The temperature dependence measurement results of the devices with the P3HT thicknesses of (a) 5 and (b) $10 \mathrm{~nm}$. Left: Arrhenius plot of the temperature dependence at difference biases. The current densities were obtained by taking the logarithmic average of the current densities of all junction diameters for each P3HT thickness. Right: The distribution of $\log (|J|)$ at biases of (a) $-380 \mathrm{mV}$ and (b) $-500 \mathrm{mV}$. Full and dashed bars represent the results measured in the cooling-down and warming-up cycles, respectively. 

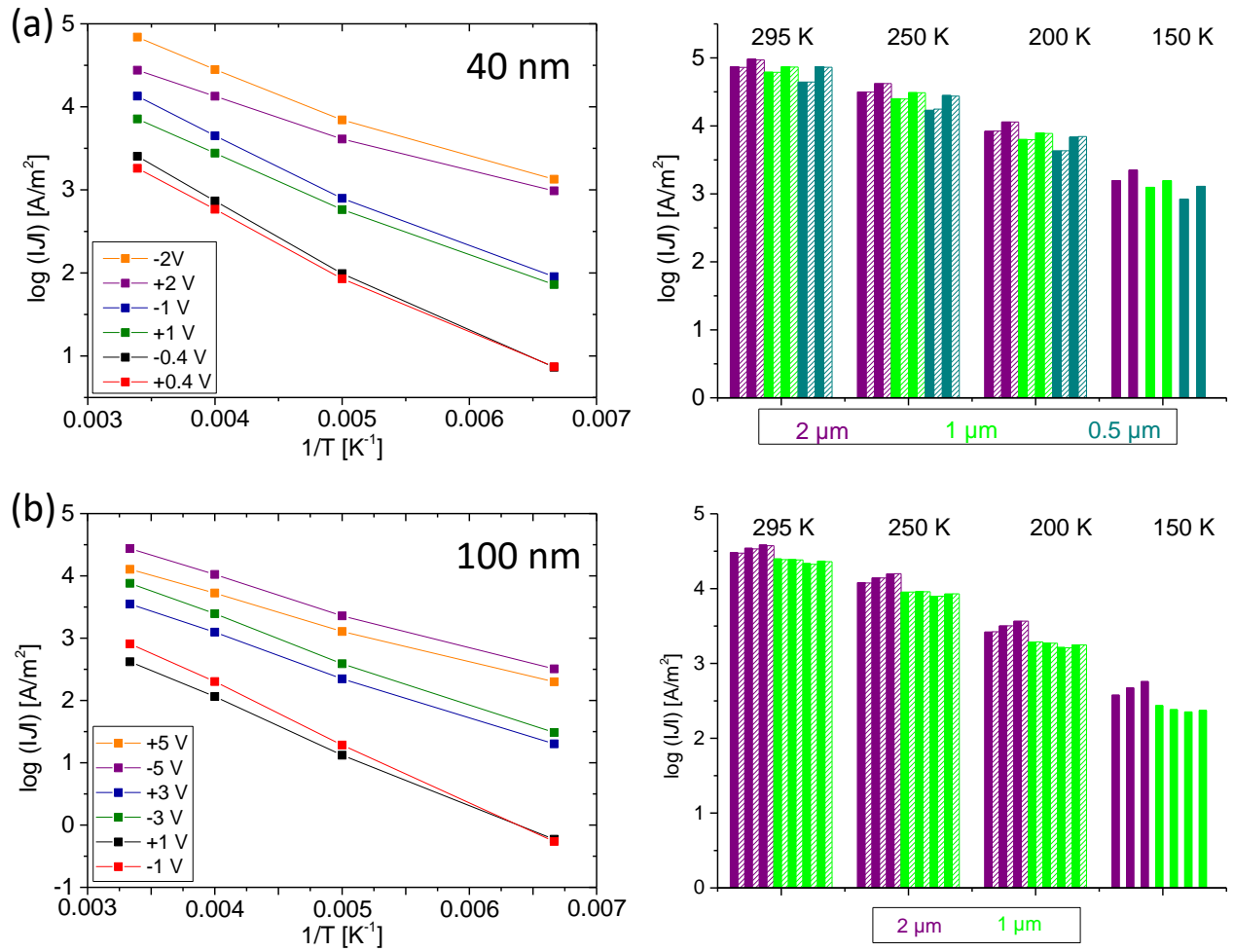

Figure 5.8: The temperature dependence measurement results of the devices with the P3HT thicknesses of (a) 40 and (b) $100 \mathrm{~nm}$. Left: Arrhenius plot of the temperature dependence at difference biases. The current densities were obtained by taking the logarithmic average of all junction diameters for each P3HT thickness. Right: The distribution of $\log (|J|)$ at biases of $(a)-2 \mathrm{~V}$ and $(\mathrm{b})-5 \mathrm{~V}$. Full and dashed bars represent the results measured in the cooling-down and warming-up cycles, respectively.

\subsection{Simulation of the temperature dependence}

We simulated the temperature dependence using the drift-diffusion model described in Ref. [55]. The model makes use of a mobility function $\mu(T, n, F)$ depending on temperature $T$, and on the local charge-carrier concentration $n(x)$ and electric field $F(x)$ ( $x$ is the distance from the injection electrode), as calculated for thermally assisted hopping in between localized sites with a site density $N_{t}$ and random on-site energies taken from a Gaussian density of states (DOS) with standard deviation $\sigma$. In the absence of an injection barrier, the work function of the metal is aligned with the center of the DOS and we have $n(O)=n(L)=N_{t} / 2$ as boundary conditions for the solution of the drift-diffusion equation, where $L$ is the P3HT thickness. In the presence of injection barriers $\varphi_{1}$ and $\varphi_{2}$ at the injecting and collecting contact, respectively, we obtain the boundary conditions $n(0)$ and $n(L)$ by evaluating the 
Gauss-Fermi integral. Three parameters were derived from the simulations, the room temperature zero-concentration zero-field hopping mobility $\mu_{0}=\mu(294 \mathrm{~K}, 0,0)$, the site density $N_{t}$, and the width of the Gaussian DOS $\sigma$. P3HT dielectric constant $\left(\varepsilon_{r}=4.4\right)$ was taken from Ref. [56].

We only simulated the data in the negative voltage region because of a slightly lower injection barrier. In addition, only devices with $2 \mu \mathrm{m}$ P3HT diameter were simulated, considering the possible RIE etching effect mentioned above. We concentrated on the devices with $100 \mathrm{~nm}$ P3HT thickness, for which the continuum drift-diffusion model is expected to work best, and for which the spread in the data is the least, as shown in Fig. 5.4.

Firstly, zero injection barrier was assumed. When the parameters were set to: $\mu_{0}=7 \times 10^{-5}$ $\mathrm{cm}^{2} / \mathrm{Vs}$, in accordance with other (vertical) devices [57], $N_{t}=1.5 \times 10^{26} \mathrm{~m}^{-3}$ and $\sigma=0.075 \mathrm{eV}$, we obtained the best agreement of the modelled and the measured $J-V$ characteristics for the devices with the P3HT thicknesses of 100 and 40 nm, as shown in Figs. 5.9(a) and 5.9(b). The site density is of the order of that in a typical organic semiconductor, and the width of the Gaussian DOS is also reasonable for the disordered organic materials [55]. For the devices with the P3HT thicknesses of 10 and $5 \mathrm{~nm}$, the simulations do not fit the data (Figs. 5.9(c) and 5.9(d)). The main discrepancy is that the simulated results are larger than the experimental results. Nonetheless, the measured temperature dependence is still well described in the simulated results.

We tried lowering down the modelled results of the devices with smaller P3HT thicknesses by increasing the injection barrier to $0.1 \mathrm{eV}$ and $0.2 \mathrm{eV}$, to get agreement of the modelled and the measured results. We found that when taking account of the injection barriers, the simulated temperature dependence deviated from the measured results, especially for the devices with $5 \mathrm{~nm}$ P3HT thickness. Specifically, the injection barriers strengthened the temperature dependence, as shown in Figs 5.10 and 5.11. Adding an injection barrier leads to an activated transport with the injection barrier as the activation energy, therefore enhances the temperature dependence.

Based on the above simulation results, we conclude that the injection barrier is not more than $0.1 \mathrm{eV}$ in the Au-P3HT-Au devices. The low injection barrier can also explain the weaker temperature dependence of the devices with smaller P3HT thicknesses. When the barrier is low, the injection of charge carriers is very good even at low temperatures, which leads to a high carrier density near the electrode where both diffusive transport away from the electrode and drift transport toward to the electrode contribute to the net charge transport [55]. In the devices with thinner $\mathrm{P} 3 \mathrm{HT}$, the electrodes are very close to each other, the diffusion transport dominants the transport between the electrodes with a high carrier 
density distribution across the $\mathrm{P} 3 \mathrm{HT}$. In the devices with thicker $\mathrm{P} 3 \mathrm{HT}$, the carrier density in the device center is much smaller than that near the electrode [55]. The much higher average carrier density in the thinner devices leads to a much weaker temperature dependence, in accordance with the theoretical prediction [58].
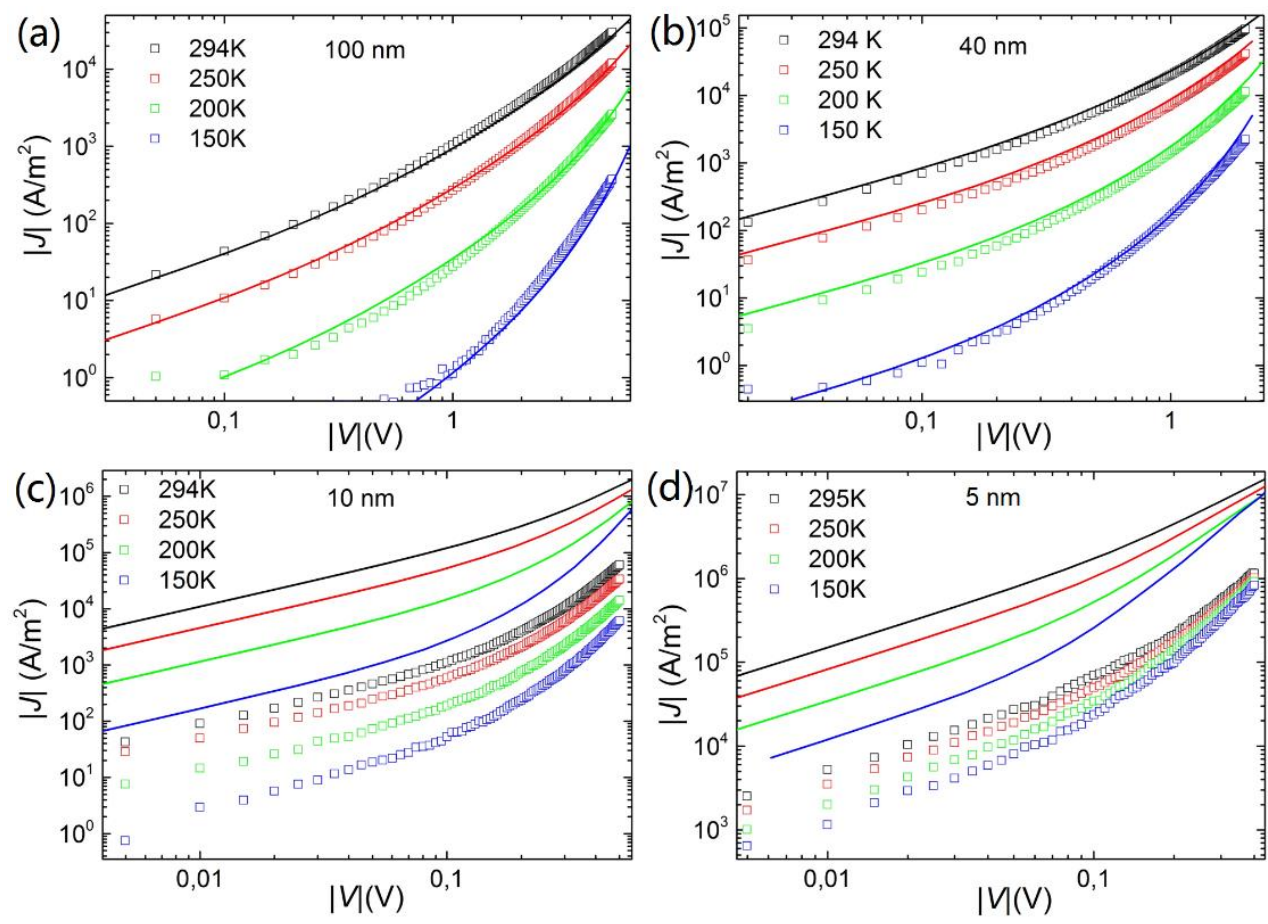

Figure 5.9: Experimental (symbols) and modelled (lines) $J-V$ characteristics of the devices with $2 \mu \mathrm{m}$ P3HT diameter and (a) $100 \mathrm{~nm}$, (b) $40 \mathrm{~nm}$, (c) $10 \mathrm{~nm}$ and (d) $5 \mathrm{~nm}$ P3HT thickness at various temperatures. The injection barrier was set to $0 \mathrm{eV}$ for the modeling. Other parameters in the drift-diffusion modeling are: room-temperature (294 K) zero-concentration zero-field mobility $\mu_{0}=7 \times 10^{-5} \mathrm{~cm}^{2} / \mathrm{Vs}$, site density $N_{t}=1.5 \times 10^{26} \mathrm{~m}^{-3}$, width of the Gaussian DOS $\sigma=75 \mathrm{meV}$. The relative dielectric constant of the P3HT $\varepsilon_{r}=4.4$. 

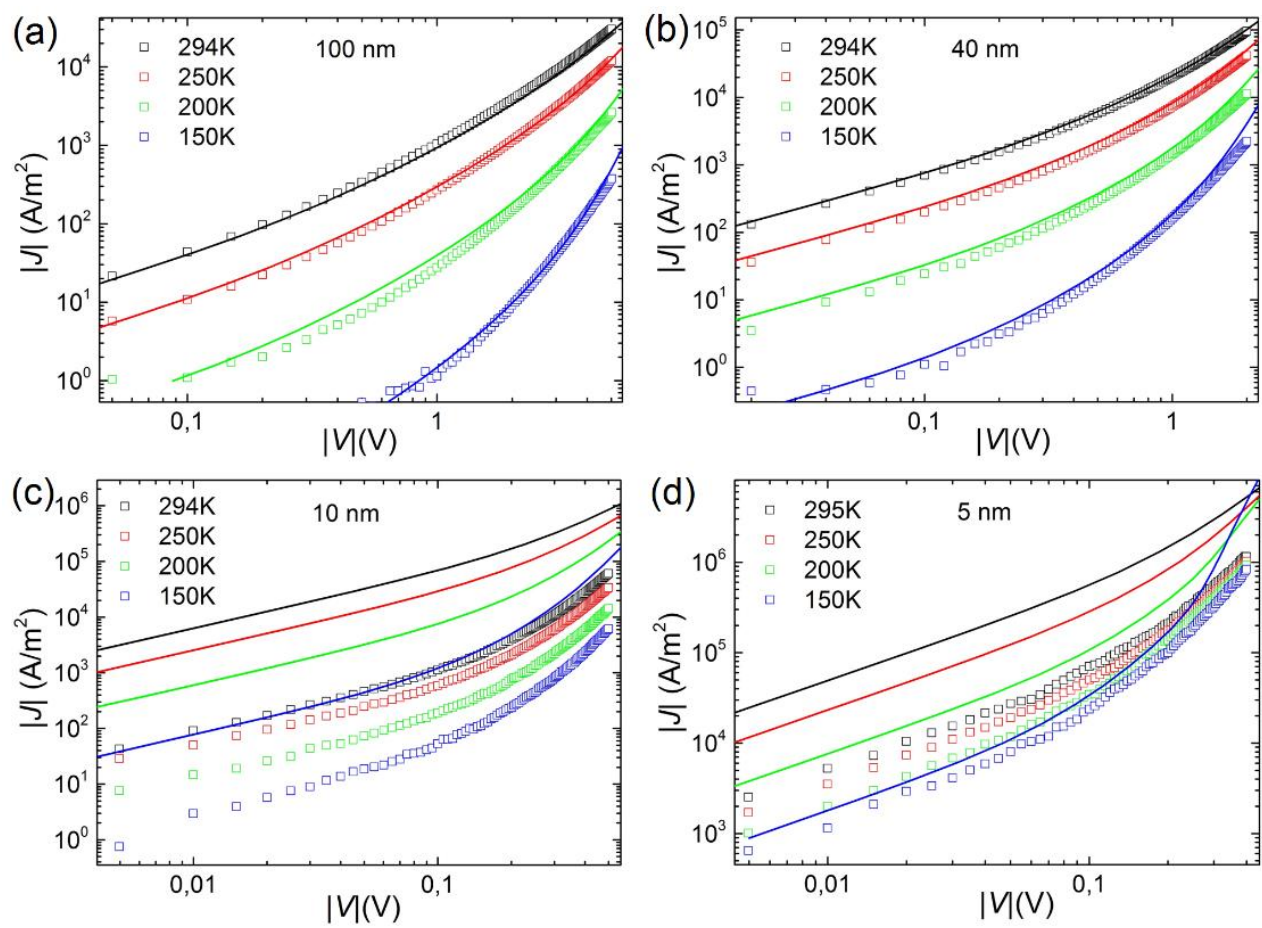

Figure 5.10: Experimental (symbols) and modelled (lines) $\mathrm{J}-\mathrm{V}$ characteristics of the devices with $2 \mu \mathrm{m}$ P3HT diameter and (a) $100 \mathrm{~nm}$, (b) $40 \mathrm{~nm}$, (c) $10 \mathrm{~nm}$ and (d) $5 \mathrm{~nm}$ P3HT thickness at various temperatures. The injection barrier was set to $0.1 \mathrm{eV}$ for the modeling. Other parameters in the drift-diffusion modeling are: room-temperature (294 K) zero-concentration zero-field mobility $\mu_{0}=7.3 \times 10^{-5} \mathrm{~cm}^{2} / \mathrm{Vs}$, site density $N_{t}=1.2 \times 10^{26} \mathrm{~m}^{-3}$, width of the Gaussian DOS $\sigma=72.5 \mathrm{meV}$. The relative dielectric constant of the P3HT $\varepsilon_{r}=4.4$. 

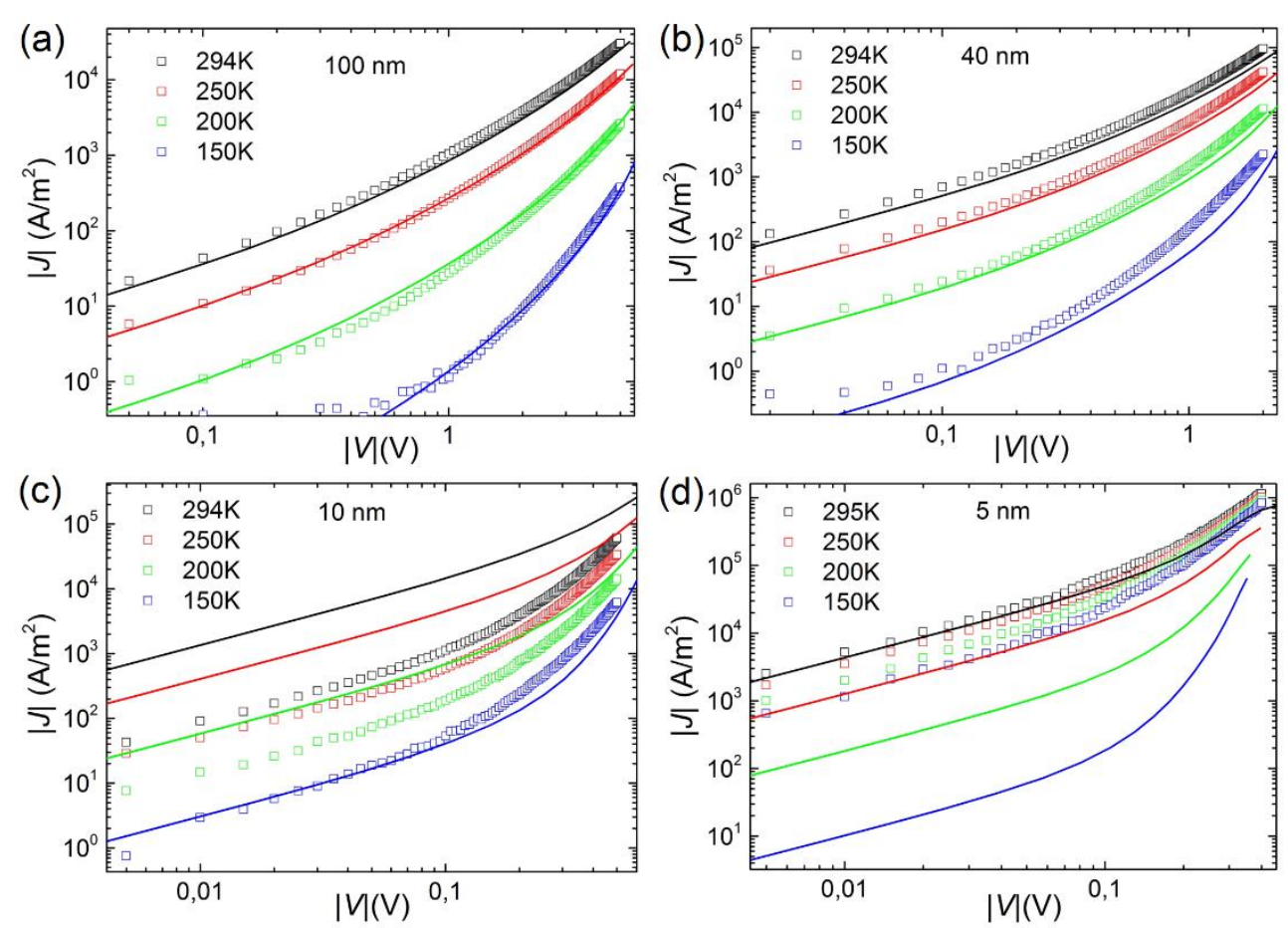

Figure 5.11: Experimental (symbols) and modelled (lines) $\mathrm{J}-\mathrm{V}$ characteristics of the devices with $2 \mu \mathrm{m}$ P3HT diameter and (a) $100 \mathrm{~nm}$, (b) $40 \mathrm{~nm}$, (c) $10 \mathrm{~nm}$ and (d) $5 \mathrm{~nm}$ P3HT thickness at various temperatures. The injection barrier was set to $0.2 \mathrm{eV}$ for the modeling. Other parameters in the drift-diffusion modeling are: room-temperature (294 K) zero-concentration zero-field mobility $\mu_{0}=8 \times 10^{-5} \mathrm{~cm}^{2} / \mathrm{Vs}$, site density $N_{t}=1.0 \times 10^{26} \mathrm{~m}^{-3}$, width of the Gaussian DOS $\sigma=71.25 \mathrm{meV}$. The relative dielectric constant of the P3HT $\varepsilon_{r}=4.4$.

We also considered a possible error in the nominal P3HT thicknesses. To decrease the simulation results, we had to increase the nominal thickness in the simulations. We found that increasing the nominal thickness led to enhancement of the temperature dependence. For the device with $5 \mathrm{~nm}$ P3HT thickness, we had to go to a thickness of $13 \mathrm{~nm}$ to get a reasonable agreement for the room-temperature $J-V$ curve, but the simulated temperature dependence was then much too strong. Hence, we conclude that it is not possible to attribute the discrepancy between the simulated and measured results to an error in the film thickness.

The most reasonable explanation seems to be the ordering of the P3HT close to the $\mathrm{Au}$ electrodes. It has been reported that the first few layers of P3HT on a silicon oxide substrate often have a higher amount of edge-on orientation as compared to the bulk, which is composed of randomly dispersed $\pi$-stacked aggregates [59]. The edge-on orientation facilitates charge transport in the direction along the substrate, but severely impedes it in 
the vertical direction. In the case that also on Au electrodes in our devices the first few nanometers have an edge-on orientation, the conductivity of the devices with $10 \mathrm{~nm}$ and 5 $\mathrm{nm}$ P3HT thickness would be much less than in the simulations, in accordance with our finding. Edge-on orientation of $\mathrm{P} 3 \mathrm{HT}$ at the interface with Au after an annealing procedure has indeed been reported $[60,61]$. For the thicker devices the effect will be much weaker, because the conductivity is then governed by the bulk.

\subsection{Summary and outlook}

We fabricated Au-P3HT-Au vertical pillar devices by wedging transfer, utilizing the wedgetransferred Au top contacts as etch masks for directional dry etching of the P3HT thin film. SEM images showed that the $\mathrm{P} 3 \mathrm{HT}$ is well protected by the top contacts, and that there is a distinctive interface between the metal and the organic layer, suggesting that the metal does not penetrate the P3HT. Lateral dimension of the devices can be scaled down to hundreds of nanometers and the thickness of P3HT can be as small as $5 \mathrm{~nm}$. The relatively high working device yield of $40-85 \%$ indicates that the top-contacting is very soft, allowing for charge transport through very thin organic films. The junctions are robust under high current densities and reveal thermally assisted hopping transport. Simulations of the temperature dependences were performed based on the drift-diffusion model with a Gaussian DOS. Experimental results of the devices with the P3HT thicknesses of 40 and 100 $\mathrm{nm}$ can be explained by the model very well. The simulated results indicate low injection barrier (less than $0.1 \mathrm{eV}$ ) which can explain the weaker temperature dependence of the devices with thinner P3HT. However, for the devices with the P3HT thicknesses of 5 and 10 $\mathrm{nm}$, the calculated current densities are higher than the measured values. Nonetheless, the measured temperature dependence is still well described in the simulated results. We attribute the discrepancy to a different orientation of the chains in the $\mathrm{P} 3 \mathrm{HT}$ thin film close to the Au electrodes as compared to the bulk. We conclude that carrier injection in our devices is very good, yielding the prospect of new types of very thin and highly conducting organic devices. 


\section{References}

1. Zhang, L., et al., Oligothiophene Semiconductors: Synthesis, Characterization, and Applications for Organic Devices. ACS Applied Materials \& Interfaces, 2014. 6(8): p. 5327-5343.

2. Kumar, B., B.K. Kaushik, and Y.S. Negi, Organic thin film transistors: structures, models, materials, fabrication, and applications: a review. Polymer Reviews, 2014. 54(1): p. 33-111.

3. Nielsen, C.B. and I. McCulloch, Recent advances in transistor performance of polythiophenes. Progress in Polymer Science, 2013. 38(12): p. 2053-2069.

4. Sawabe, K., et al., Current-Confinement Structure and Extremely High Current Density in Organic Light-Emitting Transistors. Advanced Materials, 2012. 24(46): p. 6141-6146.

5. Klauk, H., Organic thin-film transistors. Chemical Society Reviews, 2010. 39(7): p. 2643-2666.

6. from personal communication with Prof. Karl Leo, Technical University Dresden, Germany.

7. Zhang, L., et al., Bistetracene: An Air-Stable, High-Mobility Organic Semiconductor with Extended Conjugation. Journal of the American Chemical Society, 2014. 136(26): p. 9248-9251.

8. Wang, C., et al., Semiconducting $\pi$-Conjugated Systems in Field-Effect Transistors: A Material Odyssey of Organic Electronics. Chemical Reviews, 2012. 112(4): p. 2208-2267.

9. Lüssem, B., et al., Vertical organic transistors. Journal of Physics Condensed Matter, 2015. 27(44).

10. Li, T., W. Hu, and D. Zhu, Nanogap electrodes. Advanced Materials, 2010. 22(2): p. 286-300.

11. Grünewald, M., et al., Large room-temperature magnetoresistance in lateral organic spin valves fabricated by in situ shadow evaporation. Organic Electronics: physics, materials, applications, 2013. 14(8): p. 2082-2086.

12. Chen, Y. and I. Shih, Fabrication of vertical channel top contact organic thin film transistors. Organic Electronics, 2007. 8(6): p. 655-661.

13. Liu, J., et al., Vertical polyelectrolyte-gated organic field-effect transistors. Applied Physics Letters, 2010. 97(10): p. 103303-103303-3.

14. Xiong, Z.H., et al., Giant magnetoresistance in organic spin-valves. Nature, 2004. 427(6977): p. 821-824.

15. Zhang, X., et al., Observation of a large spin-dependent transport length in organic spin valves at room temperature. Nat Commun, 2013. 4: p. 1392.

16. Fischer, A., et al., An all C60 vertical transistor for high frequency and high current density applications. Applied Physics Letters, 2012. 101(21): p. 213303.

17. Dürr, A.C., et al., Morphology and interdiffusion behavior of evaporated metal films on crystalline diindenoperylene thin films. Journal of Applied Physics, 2003. 93(9): p. 5201-5209. 
18. Cho, J.H., et al., Effects of metal penetration into organic semiconductors on the electrical properties of organic thin film transistors. Applied physics letters, 2006. 89(13): p. 132101.

19. Jia, H., et al., Patterning effects on poly (3-hexy/thiophene) organic thin film transistors using photolithographic processes. Organic Electronics, 2007. 8(1): p. 44-50.

20. Gundlach, D.J., et al., Solvent-induced phase transition in thermally evaporated pentacene films. Applied Physics Letters, 1999. 74(22): p. 3302-3304.

21. Haick, H., et al., Electrical Contacts to Organic Molecular Films by Metal Evaporation: Effect of Contacting Details. The Journal of Physical Chemistry C, 2007. 111(5): p. 2318-2329.

22. Rahim, A., et al., Metal electrode formation on organic film using xe-buffer-layerassisted deposition for efficient measurement of inelastic tunneling spectroscopy. Applied Physics Express, 2013. 6(10).

23. Haick, H. and D. Cahen, Contacting organic molecules by soft methods: towards molecule-based electronic devices. Accounts of chemical research, 2008. 41(3): p. 359-366.

24. Loo, Y.-L., et al., Electrical Contacts to Molecular Layers by Nanotransfer Printing. Nano Letters, 2003. 3(7): p. 913-917.

25. Akkerman, H.B., et al., Towards molecular electronics with large-area molecular junctions. Nature, 2006. 441(7089): p. 69-72.

26. Wan, A., et al., Reversible soft top-contacts to yield molecular junctions with precise and reproducible electrical characteristics. Advanced Functional Materials, 2014. 24(28): p. 4442-4456.

27. Vilan, A. and D. Cahen, Soft Contact Deposition onto Molecularly Modified GaAs. Thin Metal Film Flotation: Principles and Electrical Effects. Advanced Functional Materials, 2002. 12(11-12): p. 795-807.

28. Stein, N., et al., Nondestructive Contact Deposition for Molecular Electronics: SiAlkyl//Au Junctions. The Journal of Physical Chemistry C, 2010. 114(29): p. 1276912776.

29. Kleemann, H., et al., High-Performance Vertical Organic Transistors. Small, 2013. 9(21): p. 3670-3677.

30. Schneider, G.g.F., et al., Wedging Transfer of Nanostructures. Nano Letters, 2010. 10(5): p. 1912-1916.

31. Krabbenborg, S.O., et al., Symmetric Large-Area Metal-Molecular MonolayerMetal Junctions by Wedging Transfer. Advanced Functional Materials, 2013. 23(6): p. 770-776.

32. Nicolai, H.T., et al., Unification of trap-limited electron transport in semiconducting polymers. Nat Mater, 2012. 11(10): p. 882-887.

33. Lyashenko, D.A., et al., Fabrication of high-mobility poly(3-hexylthiophene) transistors at ambient conditions. Organic Electronics, 2010. 11(9): p. 1507-1510.

34. Osaka, M., et al., Development of highly conductive nanodomains in poly(3hexylthiophene) films studied by conductive atomic force microscopy. Polymer, 2013. 54(14): p. 3443-3447. 
35. Chang, J.-F., et al., Enhanced Mobility of Poly(3-hexylthiophene) Transistors by SpinCoating from High-Boiling-Point Solvents. Chemistry of Materials, 2004. 16(23): p. 4772-4776.

36. Machui, F., et al., Determination of the P3HT:PCBM solubility parameters via a binary solvent gradient method: Impact of solubility on the photovoltaic performance. Solar Energy Materials and Solar Cells, 2012. 100(0): p. 138-146.

37. Aarnio, H., et al., Spontaneous Charge Transfer and Dipole Formation at the Interface Between P3HT and PCBM. Advanced Energy Materials, 2011. 1(5): p. 792797.

38. Osikowicz, W., et al., Energetics at Au top and bottom contacts on conjugated polymers. Applied Physics Letters, 2006. 88(19): p. 193504.

39. Zhao, Y., Y. Guo, and Y. Liu, 25th Anniversary Article: Recent Advances in n-Type and Ambipolar Organic Field-Effect Transistors. Advanced Materials, 2013. 25(38): p. 5372-5391.

40. Blakesley, J.C., et al., Towards reliable charge-mobility benchmark measurements for organic semiconductors. Organic Electronics, 2014. 15(6): p. 1263-1272.

41. Wilbers, J.G., Vertical hybrid inorganic-organic nanoelectronic devices. PhD thesis, Univeristy of Twente, 2016, Gildeprint.

42. Büyükköse, S., B. Vratzov, and W.G. van der Wiel, High-quality global hydrogen silsequioxane contact planarization for nanoimprint lithography. Journal of Vacuum Science \&amp; Technology B, 2011. 29(2): p. -.

43. Schouten, R., QT designed instrumentation, http://qt.tn.tudelft.nl/ schouten/ (Date of access: 28-11-2016)

44. Yaffe, O., et al., Hg/Molecular Monolayer-Si Junctions: Electrical Interplay between Monolayer Properties and Semiconductor Doping Density. The Journal of Physical Chemistry C, 2010. 114(22): p. 10270-10279.

45. Valkenier-van Dijk, E.H., Molecular conductance: synthesis, self-assembly, and electrical characterization of alpha-conjugated wires and switches. 2011, University of Groningen.

46. Liao, K.-C., et al., Fluorination, and Tunneling across Molecular Junctions. Journal of the American Chemical Society, 2015. 137(11): p. 3852-3858.

47. Thuo, M.M., et al., Odd-Even Effects in Charge Transport across Self-Assembled Monolayers. Journal of the American Chemical Society, 2011. 133(9): p. 2962-2975.

48. Tanase, C., et al., Unification of the hole transport in polymeric field-effect transistors and light-emitting diodes. Physical Review Letters, 2003. 91(21): p. 216601.

49. Coropceanu, V., et al., Charge Transport in Organic Semiconductors. Chemical Reviews, 2007. 107(4): p. 926-952.

50. Dudenko, D., et al., A Strategy for Revealing the Packing in Semicrystalline $\pi$ Conjugated Polymers: Crystal Structure of Bulk Poly-3-hexyl-thiophene (P3HT). Angewandte Chemie International Edition, 2012. 51(44): p. 11068-11072.

51. Tengstedt, C., et al., Fermi-level pinning at conjugated polymer interfaces. Applied Physics Letters, 2006. 88(5): p. 053502. 
52. Joseph Kline, R., M.D. McGehee, and M.F. Toney, Highly oriented crystals at the buried interface in polythiophene thin-film transistors. Nat Mater, 2006. 5(3): p. 222-228.

53. Li, C., et al., Universal trap effect in carrier transport of disordered organic semiconductors: transition from shallow trapping to deep trapping. The Journal of Physical Chemistry C, 2014. 118(20): p. 10651-10660.

54. Roichman, Y. and '. רויכמן, Charge transport in conjugated polymers. 2004: Technion-Israel Institute of Technology, Faculty of Electrical Engineering.

55. Van Mensfoort, S. and R. Coehoorn, Effect of Gaussian disorder on the voltage dependence of the current density in sandwich-type devices based on organic semiconductors. Physical Review B, 2008. 78(8): p. 085207.

56. Cho, Y.-S. and R.R. Franklin, Conducting polymer material characterization using high frequency planar transmission line measurement. Transactions on Electrical and Electronic Materials, 2012. 13(5): p. 237-240.

57. Johnston, D.E., et al., One-volt operation of high-current vertical channel polymer semiconductor field-effect transistors. Nano letters, 2012. 12(8): p. 4181-4186.

58. Pasveer, W., et al., Unified description of charge-carrier mobilities in disordered semiconducting polymers. Physical review letters, 2005. 94(20): p. 206601.

59. Joshi, S., et al., Thickness Dependence of the Crystalline Structure and Hole Mobility in Thin Films of Low Molecular Weight Poly(3-hexylthiophene). Macromolecules, 2008. 41(18): p. 6800-6808.

60. Park, Y.D., The Molecular Structures of Poly (3-hexylthiophene) Films Determine the Contact Properties at the Electrode/Semiconductor Interface. Bulletin of the Korean Chemical Society, 2014. 35(8): p. 2277-2280.

61. Gurau, M.C., et al., Measuring Molecular Order in Poly(3-alkylthiophene) Thin Films with Polarizing Spectroscopies. Langmuir, 2007. 23(2): p. 834-842. 


\section{Chapter 6}

\section{Vertical P3HT field-effect transistors}

\subsection{Introduction and motivation}

In this chapter, we further discuss gated $\mathrm{P} 3 \mathrm{HT}$ pillar devices. To realize gate control of the electrical transport of the two-terminal devices discussed in the last chapter, we fabricated aluminum gate electrodes around the $\mathrm{P} 3 \mathrm{HT}$ pillars with $\mathrm{Al}_{2} \mathrm{O}_{3}$ as gate dielectric between the gate electrode and the $\mathrm{P} 3 \mathrm{HT}$ pillar. We performed electrical transport measurements in these novel devices. Numerical device simulations were executed as well.

\subsection{Device fabrication}

In this section, the fabrication process of the gated devices is briefly described. More details of the process flow are elucidated in Ref. [1]. The whole fabrication process is schematically shown in Fig. 6.1. Similar to the fabrication process described in Chapter 5, we employed the wedging transfer technique and oxygen plasma reactive ion etching (RIE) to prepare P3HT pillars with palladium top contacts (Figs. 6.1(a)-(d)). The P3HT thickness of the gated devices was $100 \mathrm{~nm}$, and the pillars had the same diameters as in Chapter 5: $d=2 \mu \mathrm{m}, 1 \mu \mathrm{m}$, $500 \mathrm{~nm}$ and $200 \mathrm{~nm}$. The main difference with the process described in the last chapter is that the $\mathrm{Al}_{2} \mathrm{O}_{3}$ gate dielectric and aluminum gate electrodes were fabricated before placing the final top electrodes. The $\mathrm{Al}_{2} \mathrm{O}_{3}$ gate dielectric was deposited by atomic layer deposition (ALD) (Fig. 6.1(e)). The aluminum gate electrodes were evaporated and patterned by photolithography (Fig. 6.1(f)).

During the aluminum evaporation, we found that when the sample straightly faced the evaporation source, the overlap of the gate dielectric and the gate electrode around the pillars was very small, as shown in Fig. 6.2(a). The top Pd contact acts as a shadow mask and suppresses the deposition of aluminum in the region closely around the gate dielectric. Hence, we tilted the sample by $45^{\circ}$ such that the aluminum was evaporated from one side. Using this method, we found that the overlap became larger, as shown in Fig. 6.2(b). The disadvantage is that the gate electrode does not contact the gate dielectric at the other side of the pillar. In the following, the former device type is called "full-gate" device and the latter type is called "half-gate" device. 
(a)

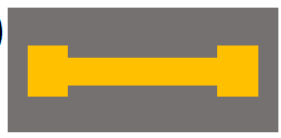

bottom electrodes

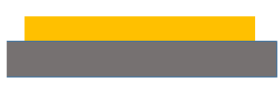

(d)

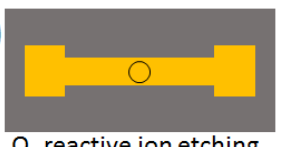

$\mathrm{O}_{2}$ reactive ion etching
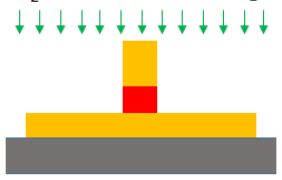

(g)

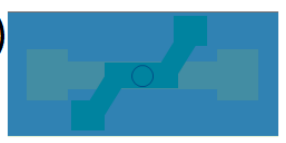

HSQ planarization
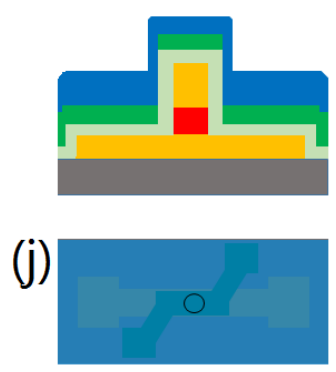

$2^{\text {nd }} \mathrm{HSQ}$ planarization

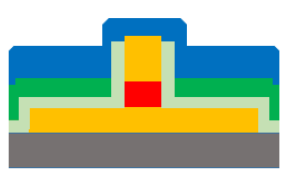

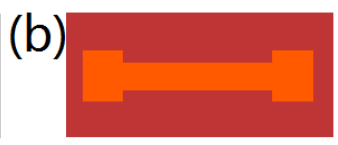

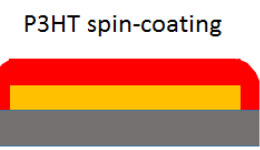

(e)
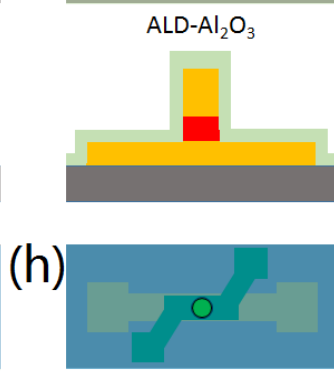

$\mathrm{CHF}_{3}$ reactive ion etching $\downarrow \downarrow \downarrow \downarrow \downarrow \downarrow \downarrow \downarrow \downarrow \downarrow \downarrow \downarrow \downarrow$

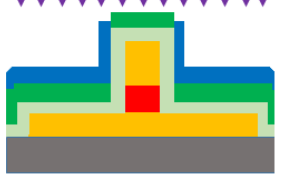

(k)

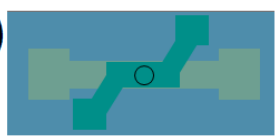

$\mathrm{CHF}_{3}$ reactive ion etching

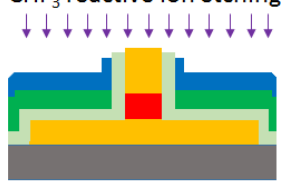

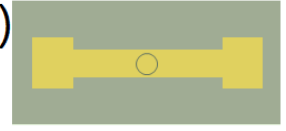

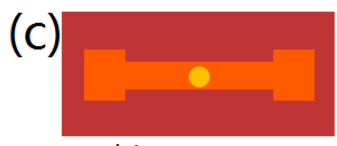

wedging transfer

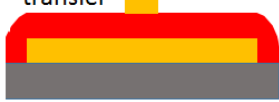

(f)

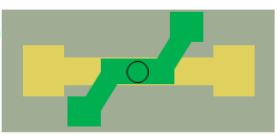

gate electrodes

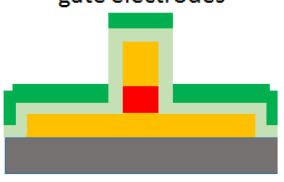

(i)

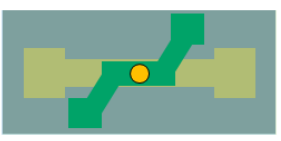

Ar mechanical ion etching

$\downarrow \downarrow \downarrow \downarrow \downarrow \downarrow \downarrow \downarrow \downarrow \downarrow \downarrow \downarrow \downarrow$

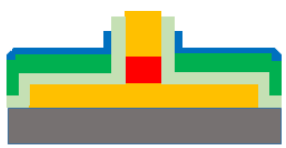

(I)

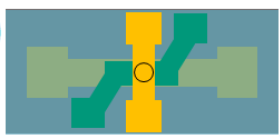

large top electrodes
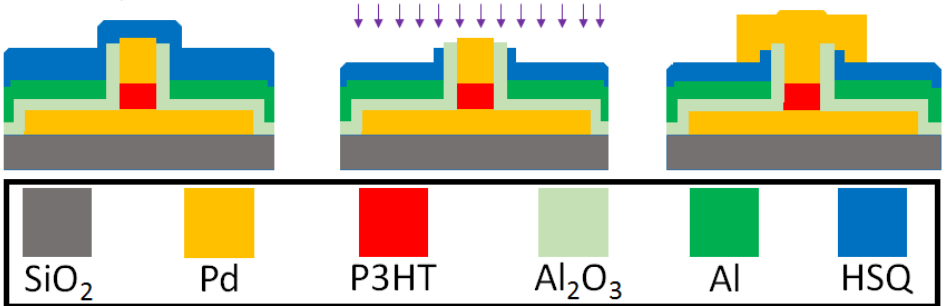

Figure 6.1: Fabrication process flow of the gated devices. In each subfigure, the top is a top view and the bottom is a side view of the devices. (a) Fabrication of bottom electrodes (Pd, with Ti as adhesion layer) on $\mathrm{SiO}_{2}$ substrates by photolithography. (b) Spin-coating of P3HT. (c) Wedging transfer of EBL patterned Pd disks. (d) RIE with $\mathrm{O}_{2}$ ions for pillar formation. (e) $\mathrm{ALD}$ of $\mathrm{Al}_{2} \mathrm{O}_{3}$. (f) Al gate electrodes patterned by photolithography. (g) Spin-coating of HSQ. (h) $\mathrm{CHF}_{3} \mathrm{RIE}$ to open the $\mathrm{Al}$ and $\mathrm{Al}_{2} \mathrm{O}_{3}$ on top of the Pd top contacts. (i) Ar mechanical ion etching to remove the $\mathrm{Al}$, $\mathrm{Al}_{2} \mathrm{O}_{3}$, and some HSQ as well, to open the Pd top contacts. (j) Second HSQ spin-coating; (k) $\mathrm{CHF}_{3} \mathrm{RIE}$ to open the Pd top contacts. (I) Patterning of large top electrodes by photolithography and RIE. 

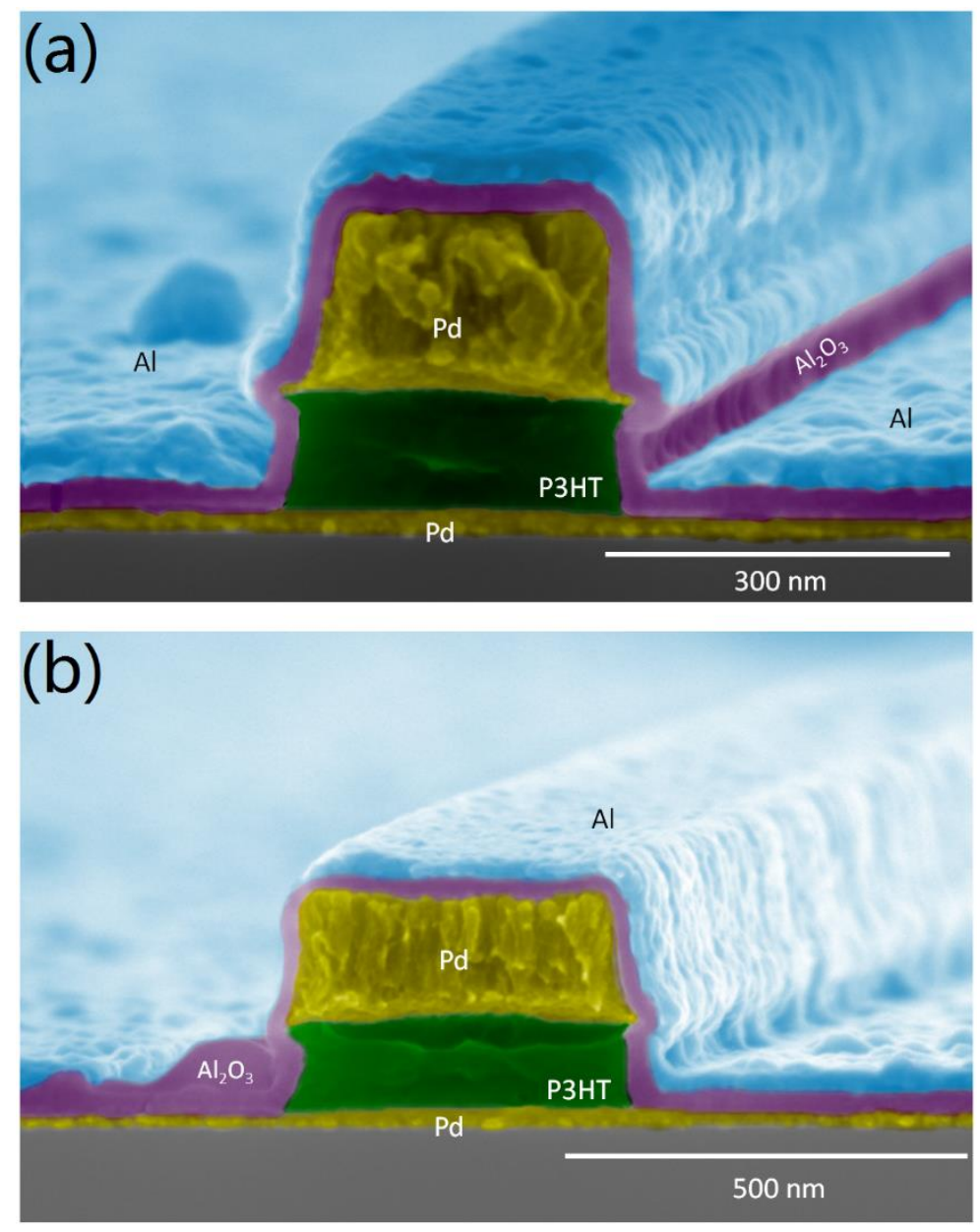

Figure 6.2: Colored SEM images showing cross sections of the full-gate (a) and the half-gate (b) test devices: $\mathrm{P} 3 \mathrm{HT}$ (100 nm, green), $\mathrm{Pd}$ top electrode (150 nm, yellow), $\mathrm{Pd}$ bottom electrode ( $20 \mathrm{~nm}$, yellow), $\mathrm{Al}_{2} \mathrm{O}_{3}$ gate dielectric ( $25 \mathrm{~nm}$, purple), and Al gate electrode $(30 \mathrm{~nm}$, blue).

After fabrication of the aluminum gate electrodes, hydrogen silsesquioxane (HSQ) was spincoated to planarize the sample surface (Fig. 6.1(g)). After this, $\mathrm{CHF}_{3} \mathrm{RIE}$ was used to remove the $\mathrm{HSQ}$, thereby uncovering the aluminum on top of the pillars (Fig. 6.1(h)). In the following step, the $\mathrm{Al}$ and $\mathrm{Al}_{2} \mathrm{O}_{3}$ on top of the pillars were mechanically etched by reactive ion beam etching (RIBE) with Ar ions (Fig. 6.1(i)) so that the top Pd contacts were exposed. After this step, conductive-probe AFM was used to check if the Pd top contacts were exposed. Once the exposure was verified, we spin-coated a new HSQ layer (Fig. 6.1(j)) since the first HSQ layer was already very thin and not well insulating after the above two steps of dry etching. Back-etching of the new HSQ layer was performed to uncover the Pd top contacts (Fig. 6.1(k)). After this step, conductive-probe AFM was used again to check if the HSQ on top of 
the Pd top contacts was etched clean. Finally, large top electrodes were deposited and patterned by photolithography and mechanically by RIBE (Fig. 6.1(I)).

\subsection{Electrical transport measurements}

The electrical transport properties of the devices were measured at room temperature (294 $\mathrm{K})$ in a turbo-pumped vacuum $\left(\sim 10^{-5}-10^{-6} \mathrm{mbar}\right)$ to suppress the air instability mentioned in Chapter 5. We used a probe station connected with an Agilent B2912A precision source/measure unit. The bottom Pd electrodes were set as the source electrodes, which were always the common ground.

Figure 6.3 shows the transport characteristics of a half-gate device with $d=1 \mu \mathrm{m}$ P3HT diameter. In Fig. 6.3(a), the top shows a measurement of the drain current $I_{D}$ when sweeping the source-drain voltage and alternating the gate-source voltage $V_{G S}$ between $10 \mathrm{~V}$ and -10 $V$. The top of Fig. $6.3(\mathrm{~b})$ shows $I_{\mathrm{D}}$ as a function of $V_{\mathrm{GS}}$, at different drain-source voltage $V_{\mathrm{DS}}$. The bottom panels in Fig. 6.3 show the corresponding gate leakage measurements. The results do not show a distinct gate effect, apart from a small drift of $I_{D}$ when sweeping $V_{G S}$ and keeping $V_{D S}$ constant (Fig. 6.3(b)). All full-gate and most half-gate devices show similar results. We also tried exchanging the source and drain electrodes, that is, grounding the top Pd electrodes. No distinct gate effect was observed.

We also observed somewhat different $I_{D}-V_{G S}$ results in two devices, one with $d=1 \mu \mathrm{m}$ and one with $d=200 \mathrm{~nm}$, although the output properties of the two devices did not show a distinct gate effect. While measuring transfer characteristics, $I_{D}$ slightly changed with the gate voltage. Specifically, $I_{D}$ increased when $V_{G S}$ became more negative. In addition, when $V_{G S}$ was swept from negative to positive, $I_{D}$ decreased, as shown in Fig. 6.4. Linear fitting reveals that the transconductance $\left(\left(I_{\mathrm{Dmax}}-I_{\mathrm{Dmin}}\right) /\left(V_{\mathrm{GS} \max }-V_{\mathrm{GS} \max }\right)\right.$, similarly hereafter $)$ is $1 \mathrm{pS}$ for $V_{\mathrm{DS}}=-3 \mathrm{~V}$ and $3 \mathrm{pS}$ for $V_{\mathrm{DS}}=-5 \mathrm{~V}$ for the device with $d=1 \mu \mathrm{m}$ (Fig. 6.4(a)). For the device with $d=200 \mathrm{~nm}$, the transconductance is only $0.13 \mathrm{pS}$ when $V_{\mathrm{DS}}=-5 \mathrm{~V}$ (Fig. 6.4(b)). The two devices were measured again before breakdown due to a too large gate voltage applied. The weak effect was reproducible. It is not likely that this weak gate dependence is due to the drift of the drain current. As shown in Fig. 6.3(b), the drift of $I_{D}$ is always in one direction, regardless of the sweep direction of the gate voltage. It is not likely either that the weak gate dependence is due to the gate leakage, because if there would be crosstalk between the $I_{\mathrm{D}}$ and $I_{\mathrm{G}}$ measurements we should observe that $I_{\mathrm{D}}$ decreases when $I_{\mathrm{G}}$ increases due to the increase of $V_{G}$. The weak gate dependence indicates that there is probably some $p$-type channel behavior in the devices. We cannot draw a definite conclusion, however, because of the smallness of the effect and because it was only observed in two devices. 

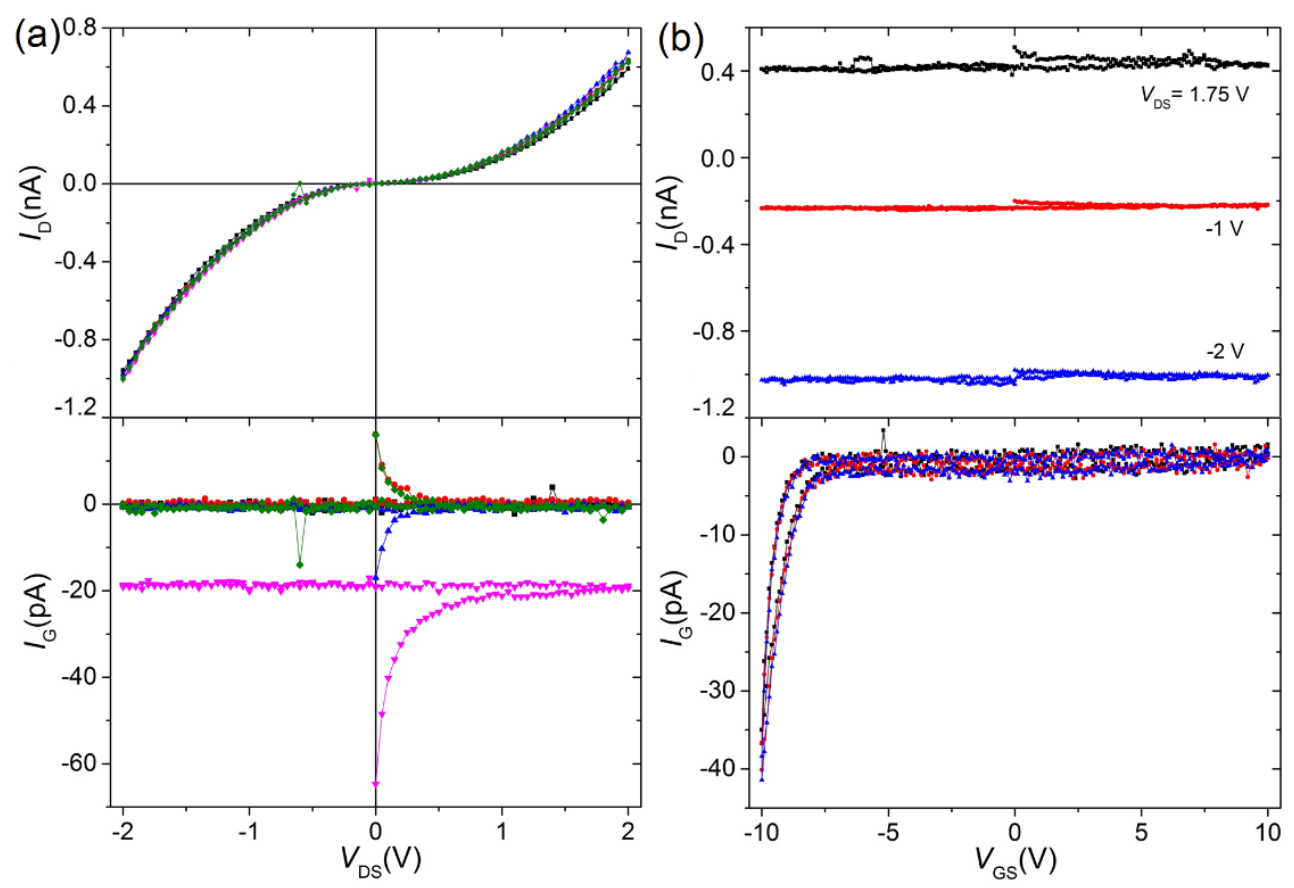

Figure 6.3: Output (top of (a)) and transfer (top of (b)) characteristics of a half-gate device with $d=\mathbf{1} \boldsymbol{\mu m}$ P3HT diameter. The bottom panels are corresponding $I_{G}$ measurement results. (a) $V_{G S}$ was set to 0 (black squares), 10 (red dots), 0 (blue up triangles), -10 (pink down triangles), and $0 \vee$ (greed diamonds), respectively. $V_{D S}$ was first swept from 0 to $2 \mathrm{~V}$, then to $-2 \mathrm{~V}$, and finally back to $0 \mathrm{~V}$. (b) $V_{D S}$ was set to $1.75 \mathrm{~V}$ (black squares), $-1 \mathrm{~V}$ (red dots), and $-2 \mathrm{~V}$ (blue up triangles), respectively. $V_{G S}$ was first swept from 0 to $10 \mathrm{~V}$, then to $-10 \mathrm{~V}$ and finally back to $0 \mathrm{~V}$.
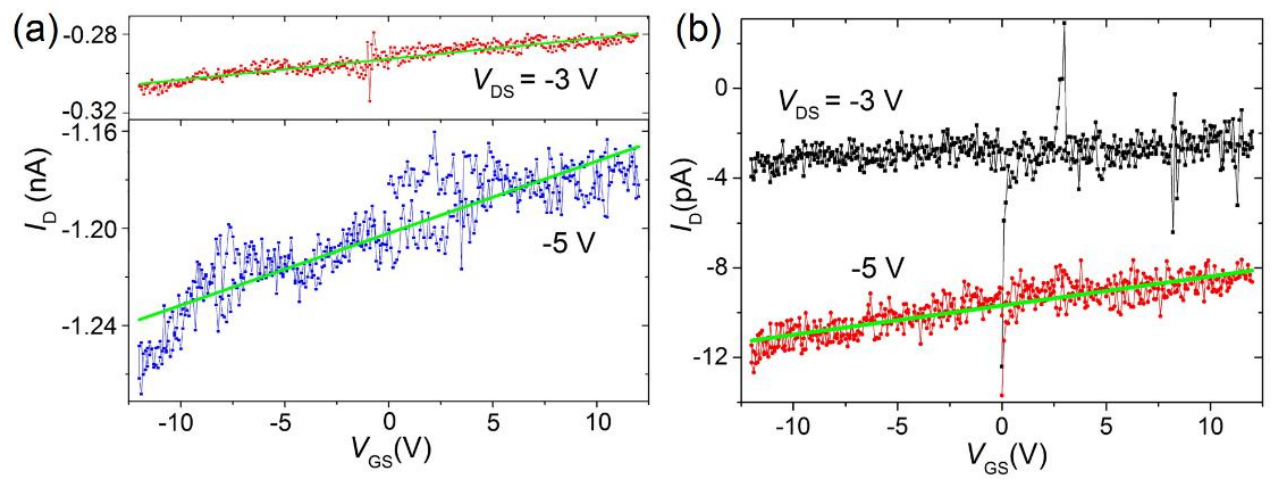

Figure 6.4: Transfer characteristics of two half-gate devices. Symbols are measured data and green lines are linear fits. (a) $d=1 \mu \mathrm{m}$. (b) $d=200 \mathrm{~nm}$. $V_{\mathrm{GS}}$ was first swept from 0 to $12 \mathrm{~V}$, then to $-12 \mathrm{~V}$, and finally back to $0 \mathrm{~V}$. The gate leakage results are similar to Fig. 6.3(b). 
In summary, neither of the full-gate and half-gate devices showed a significant gate effect. Hence, the gate effect of the P3HT pillar devices is to be greatly improved in the future. In section 6.3 we discuss device simulation results and possible reasons for the absence of a significant gate effect.

\subsection{ATLAS device simulations}

ATLAS is a commercial 2D/3D device simulation software tool developed by the company Silvaco [2]. After many extensions over the years, it can now perform various types of simulations of semiconductor devices, such as simulations of DC/AC/transients, inorganic as well as organic devices, electrical/optical/thermal properties, and so on. It also incorporates the drift-diffusion model together with a Gaussian density of states (DOS) [3], described in Chapter 5. We performed device simulations using the ATLAS software to investigate the output and transfer characteristics of the gated P3HT pillar devices.

The user interface of ATLAS is not as convenient as that of, e.g., COMSOL, where users can just point, click and input numbers to define simulations. Instead, one needs to specify "Statements" in deck files to define devices, meshes, assign models to be used for simulations, and set data to be calculated. Since ATLAS is a finite-element analysis (FEA) tool, meshing structures need to be carefully handled. Some critical regions in devices, such as interfaces between two different materials, should be meshed as fine as possible. The time scaling of ATLAS is roughly $N^{a}$, where $N$ is the number of mesh nodes and $a=2-3$, depending on the complexity of the problem [4]. Less critical regions can be coarser meshed to decrease the simulation time. Additionally, taking advantage of the symmetry of devices, which can, for example, reduce 3D to effectively 2D simulations, can dramatically increase the simulation efficiency.

Since the pillar structures are radially symmetric, we employed the $2 \mathrm{D}$ cylindrical mesh method to transfer the 3D structure to $2 \mathrm{D}$ structure in order to decrease simulation time. Figure 6.5(a) schematically shows the construction of the 2D cylindrical mesh. When a 2D rectangle is rotated by $360^{\circ}$ with one of its sides as the axis of rotation, it forms a cylinder. In this method, only the rectangle needs to be meshed. The simulation results for the rectangle are then rotated to obtain the results in the 3D device (Fig. 6.5(b)). As for the halfgate devices, although they are not radially symmetric, we assume that the gating at only side merely means a loss of part of the gate control. Hence, we used the same mesh method to simulate the half-gate devices. 


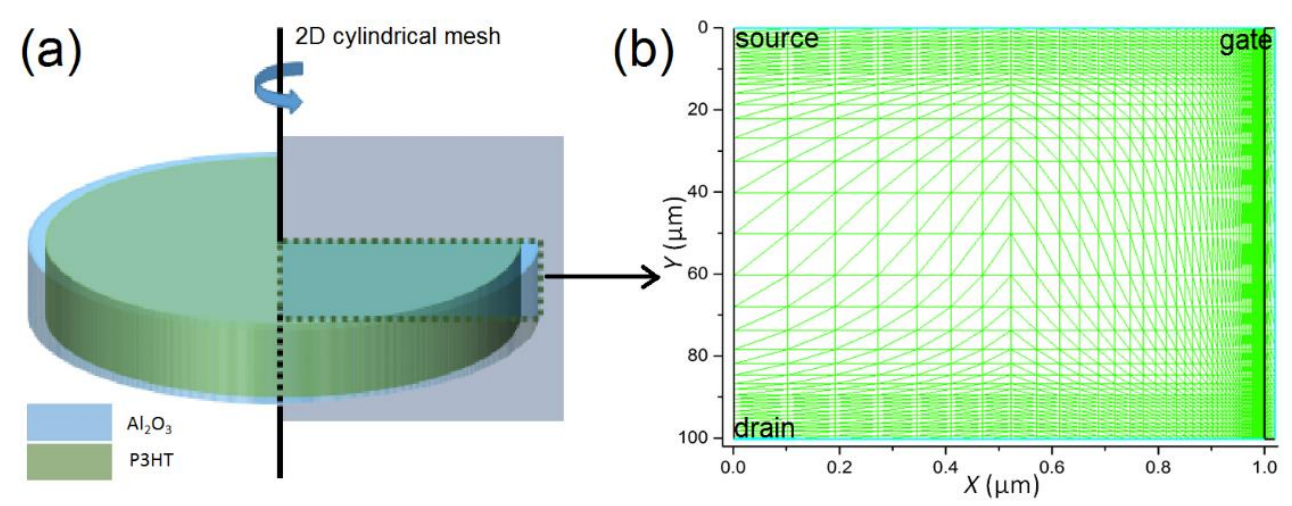

Figure 6.5: The 2D cylindrical mesh. (a) Rotation of a 2D rectangle to form a 3D cylinder. (b) Mesh grid on the 2D rectangle. The rightmost part of the rectangle is the gate dielectric; source, drain and gate electrodes were set as top, bottom and right boundaries, respectively (similarly hereafter). The $X$ axis is the distance from the center of the pillars, and the $Y$ axis is the distance from the source, which is always at $Y=0$ (similarly hereafter). The drawn situation corresponds to a 3D pillar device with P3HT diameter of $2 \mu \mathrm{m}$ and thickness of $100 \mathrm{~nm}$, and a thickness of the gate dielectric of $20 \mathrm{~nm}$. All the regions close to the interfaces were finely meshed with a $1 \mathrm{~nm}$ mesh size. Other regions were coarser meshed.

We simulated the characteristics of device with $d=200 \mathrm{~nm}$ P3HT diameter. We took $25 \mathrm{~nm}$ and $73 \mathrm{~nm}$ for the gate dielectric thickness and gate electrode height (the vertical length of the contact area between the gate electrode and the gate dielectric), respectively, according to the SEM image of the half-gate device (Fig. 6.2(b)). Since we obtained excellent agreement of the modelled and the measured results for the two-terminal devices with 100 $\mathrm{nm}$ P3HT thickness in Chapter 5, we used the same parameters in the drift-diffusion model incorporated in the ATLAS for the device simulations in this chapter. Hence, we set the room temperature zero-concentration zero-field hopping mobility $\mu_{0}$ to $7 \times 10^{-9} \mathrm{~m}^{2} / \mathrm{Vs}$, the site density $N_{t}$ to $1.5 \times 10^{26} \mathrm{~m}^{-3}$, and the width of the Gaussian DOS $\sigma$ to $0.075 \mathrm{eV}$. P3HT dielectric constant $\left(\varepsilon_{r}=4.4\right)$ was taken from Ref. [5].

The simulation results demonstrate a distinct gate effect, as shown in the simulated output and transfer characteristics (Figs. 6.6(a) and (b)). The hole concentration and hole current density distributions demonstrate that the accumulation layer thickness is only a few nanometers (Figs. 6.6(c) and (d)). The transconductance is of $\mathrm{nS}$ order, which is much larger than what we measured (Fig. 6.4(b)). Furthermore, the simulated current is also about 2 orders of magnitude larger than the measured results (Fig. 6.7(d)). Half-gate devices and full-gate devices (smaller gate electrode height than the half-gate devices, set as $10 \mathrm{~nm}$ in accordance with Fig. 6.2(a)) with different diameters were simulated as well. The simulated results all show a distinct gate effect. Different from the device with $d=200 \mathrm{~nm}$ P3HT diameter, the simulated drain currents at zero gate voltage are only a few times larger than the measured results for the devices with larger diameters, as shown in Figs. 6.7(a), (b), and 
(c). From the simulation results we conclude that there is not only a problem regarding gate effect, but also regarding the size of the conductivity of the devices, especially for the device with $d=200 \mathrm{~nm}$ P3HT diameter.
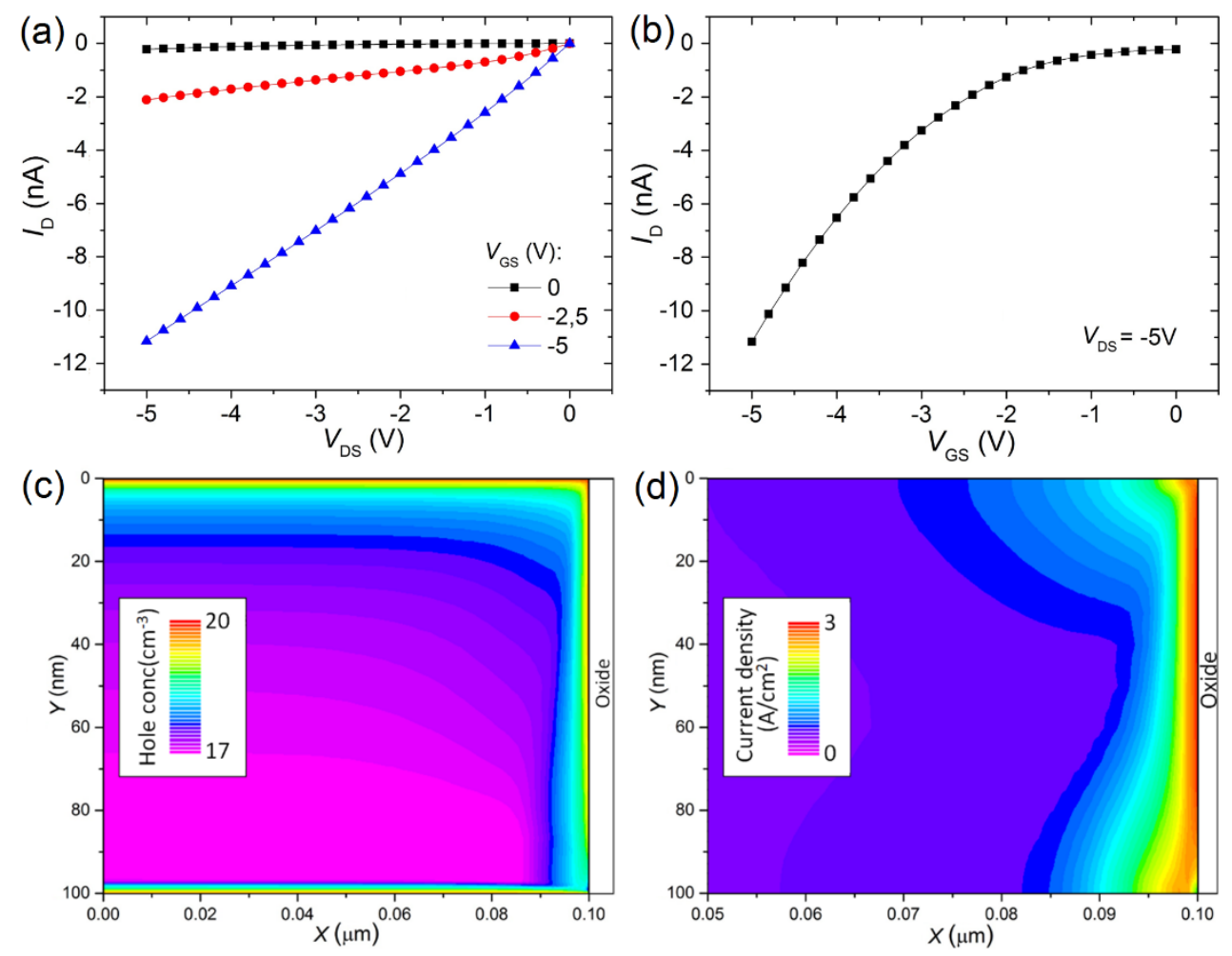

Figure 6.6: Simulation results for the device with a $200 \mathrm{~nm}$ diameter P3HT layer with $\mathbf{7 3} \mathbf{n m}$ gate electrode height and $25 \mathrm{~nm}$ oxide thickness. (a) Output characteristics for three different values of $V_{G S}$. (b) Transfer characteristics for $V_{D S}=-5 V$. (c) Hole concentration (log scale) in the whole P3HT region for $V_{G S}=V_{D S}=-5 V$. (d) Hole current density ( $\log$ scale) in the region close to the interface when $V_{G S}=V_{D S}=-5 \mathrm{~V}$. The rightmost oxide region is not completely shown here (similarly hereafter). 

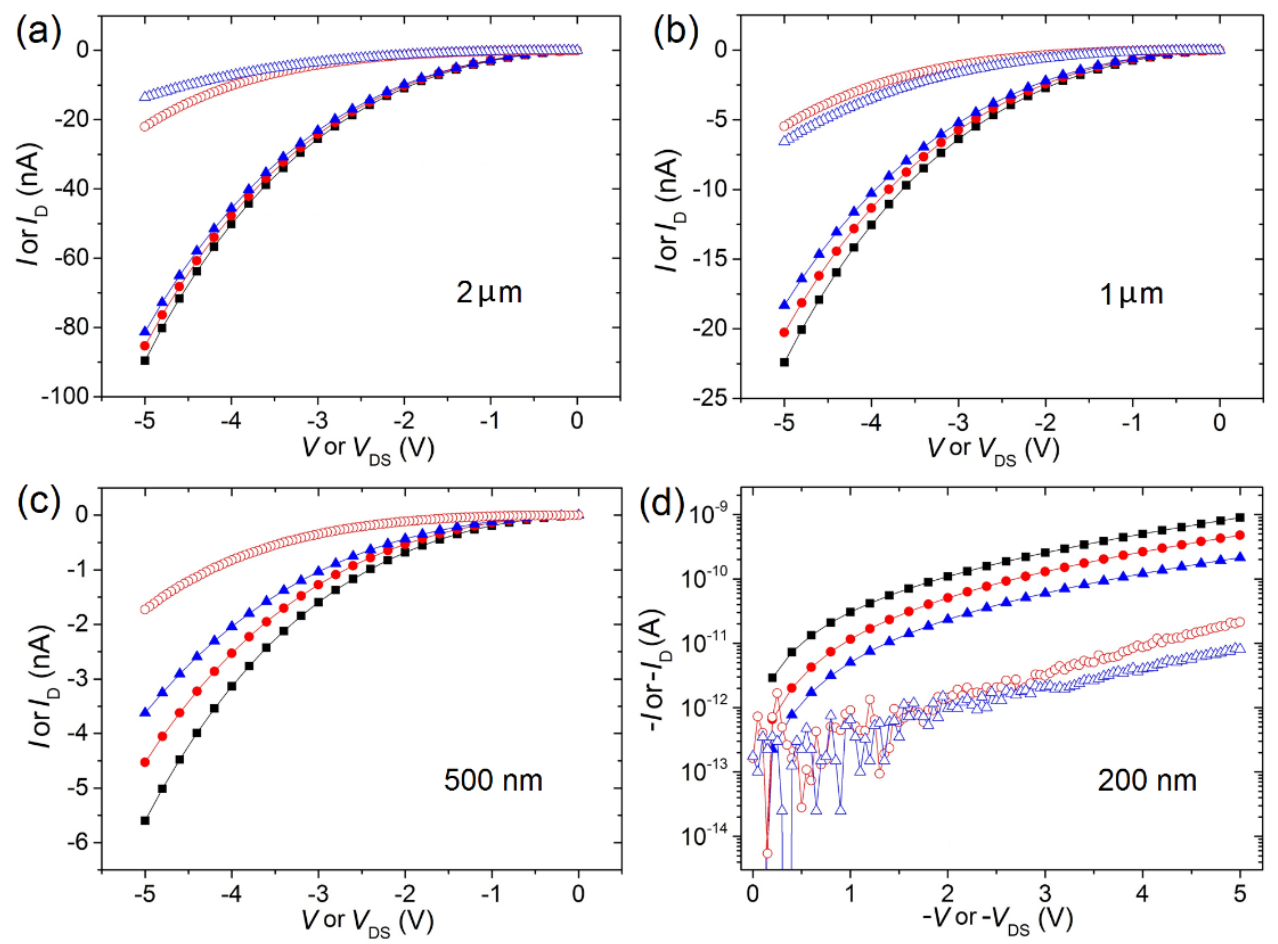

Figure 6.7: Discrepancy between simulated and measured results for full-gate and half-gate devices with four different diameters of the P3HT layers. In all of the four figures, red open circles are averaged measurement results of all full-gate devices and red solid circles are simulated results of the full-gate devices $(10 \mathrm{~nm}$ gate electrode height) at $V_{G S}=0 \mathrm{~V}$; blue open triangles are averaged measurement results of all half-gate devices and blue solid triangles are simulated results of the half-gate devices ( $73 \mathrm{~nm}$ gate electrode height) at $V_{G S}=0 \mathrm{~V}$; black solid squares are simulated results of the non-gated devices.

Since gate control of transistors is mainly caused by the generation of an accumulation layer in the region close to the semiconductor/dielectric interface, the reason for the almost absent gate effect in our devices probably lies in the region where the accumulation layer is supposed to be created. We used $\mathrm{O}_{2}$ plasma RIE to etch the $\mathrm{P} 3 \mathrm{HT}$, and it is very likely that the oxygen ions are scattered and spread out. Therefore, the outermost part of the P3HT pillars is probably damaged by the oxygen ions, leading to many dangling bonds in the damaged region. Accounting for this, we assume that there are many traps in that region. Even though charge carriers would be accumulated there by the gate field, they would not freely move. Based on this reasoning, we take a smaller room temperature zeroconcentration zero-field hopping mobility $\mu_{0}$ in a thin edge region of the $\mathrm{P} 3 \mathrm{HT}$ pillars close to the $\mathrm{P} 3 \mathrm{HT} / \mathrm{Al}_{2} \mathrm{O}_{3}$ interface, as shown in Fig. 6.8. 


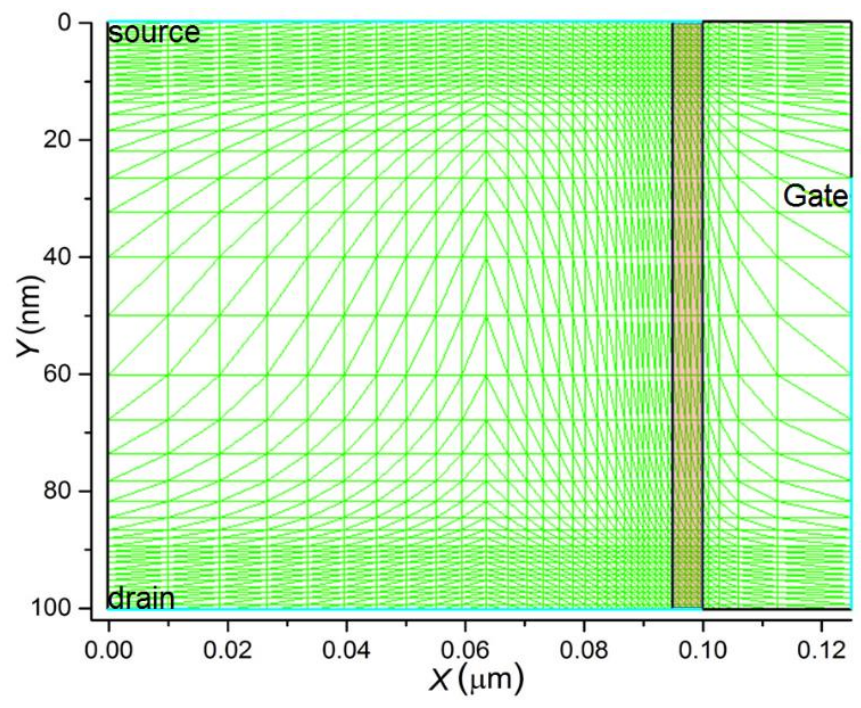

Figure 6.8: $5 \mathrm{~nm}$ thick damaged region (the shaded area) close to the interface with the oxide of a half-gate device with $d=200 \mathrm{~nm}$ P3HT diameter ( $73 \mathrm{~nm}$ gate electrode height and $25 \mathrm{~nm}$ oxide thickness). In reality, the damaged region will very likely have a different shape. We assigned a smaller mobility to the damaged region than to the undamaged P3HT. We only modulated the thickness and the mobility of the damaged region in our analysis.
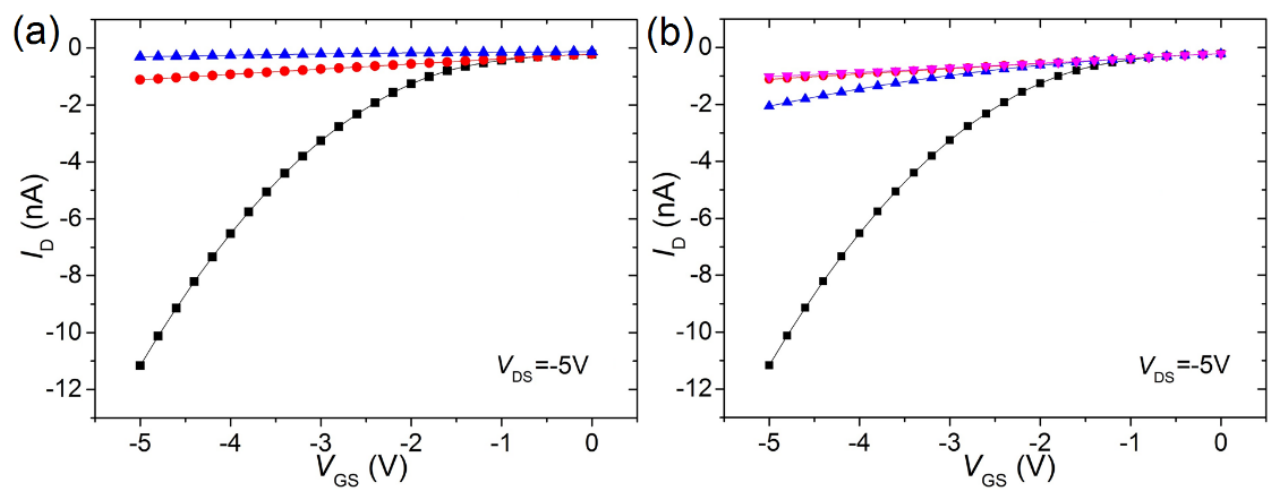

Figure 6.9: Transfer characteristics simulated with and without the damaged layers. (a) Different thicknesses of the damaged layer for a mobility in the damaged layer reduced by a factor 0.01: $0 \mathrm{~nm}$ (black squares), $5 \mathrm{~nm}$ (red dots) and $50 \mathrm{~nm}$ (blue up triangles) (b) Damaged layer with thickness of $5 \mathrm{~nm}$ and unreduced mobility (black squares), and mobilities reduced by a factor 0.1 (blue up triangles), 0.01 (red solid circles), and 0.001 (pink down triangles).

The simulation results shown in Fig. 6.9 reveal that even a $5 \mathrm{~nm}$ damaged layer with $10 \%$ of the mobility of undamaged $\mathrm{P} 3 \mathrm{HT}$ can already significantly suppress the gate effect. The hole current flow in the damaged region is suppressed, but the hole accumulation in the damaged layer is not significantly affected, as shown in Fig. 6.10. These results show that a damaged layer could be the cause for the strong suppression of the gate effect. 

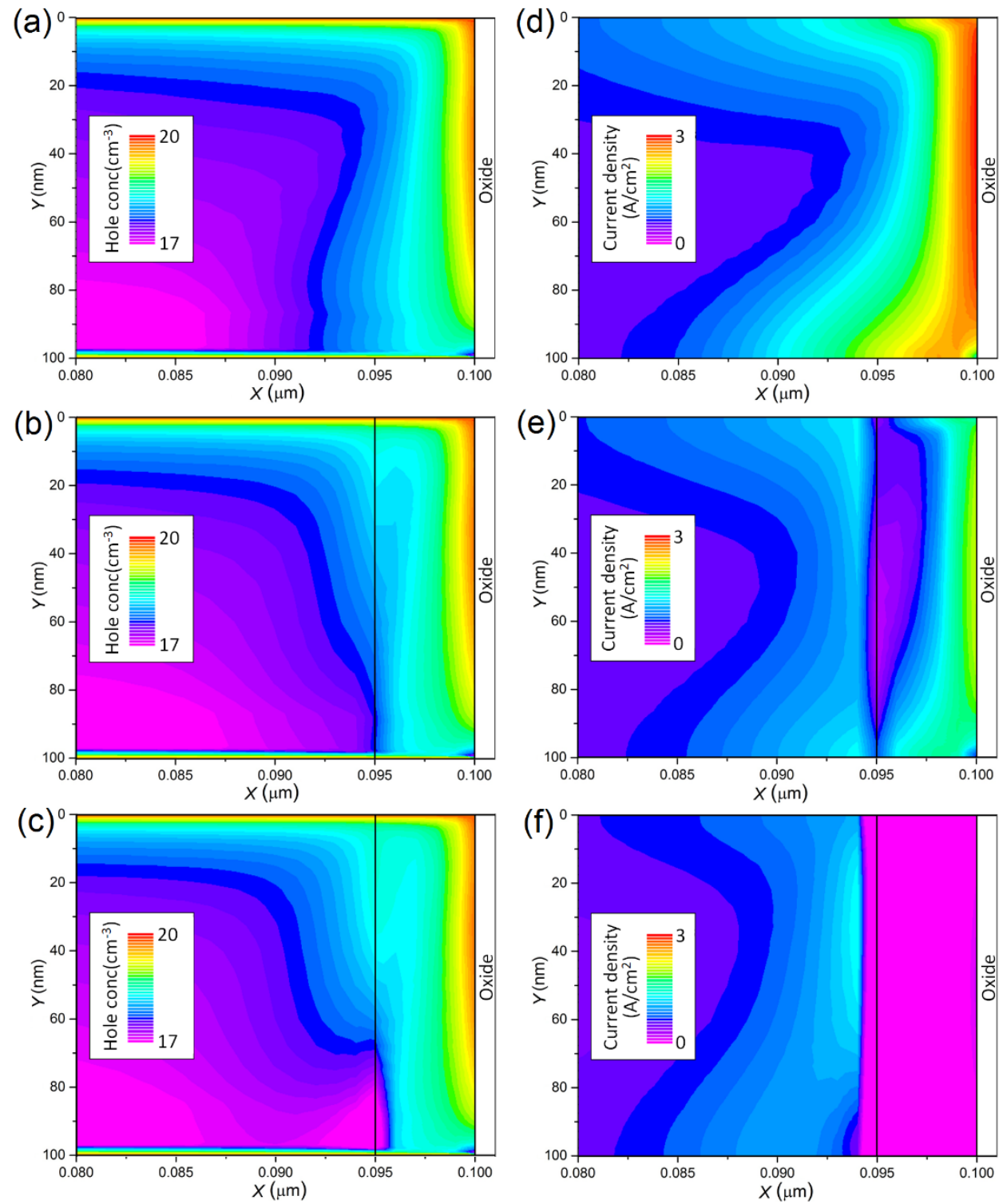

Figure 6.10: Hole concentration ((a)-(c), log scale) and hole current density ((d)-(f), log scale) in the region close to the organic/dielectric interface, for $V_{G S}=V_{D S}=-5 \mathrm{~V}$. In (a) and (d) there is no damaged layer. The damaged layer thickness is $5 \mathrm{~nm}$ for (b), (c), (e) and (f). The mobility of the damaged layer was set to 0.1 of the mobility of undamaged $\mathrm{P} 3 \mathrm{HT}$ for (b) and (e), and to 0.001 of this mobility for (c) and (f).

However, both the gate effect and the magnitude of the drain current simulated with the damaged layer, either $5 \mathrm{~nm}$ thick with $0.001 \times \mu_{0}$ or $50 \mathrm{~nm}$ thick with $0.01 \times \mu_{0}$, are still larger than the experimental results. Therefore, we fixed the decreased mobility at a very low value $\left(10^{-10} \times \mu_{0}\right)$ to assume that the damaged part was completely insulating, then tweaked 
the thickness of the damaged layer to see when the simulated transconductance matches the experimental results. The simulation results reveal that, for the device with $d=200 \mathrm{~nm}$ $\mathrm{P} 3 \mathrm{HT}$ diameter, when the damaged layer is $81 \mathrm{~nm}$ thick, the transconductance drops to about $0.9 \mathrm{pS}$ which is close to the experimental result. Besides, the magnitude of the current is close to the measured result as well, as shown in Fig. 6.11. Both the weak gate effect and the small current are described when using the thick damaged layer. We did similar simulations for the devices with $1 \mu \mathrm{m}$ P3HT diameter. We find that the damaged thickness has to be about $200 \mathrm{~nm}$ to obtain simulation results for the gate effect and the size of the current that are roughly equal to the measured data. As for the devices with $d=2 \mu \mathrm{m}$ and $500 \mathrm{~nm}$, we find that the damaged thickness has to be about $500 \mathrm{~nm}$ and $100 \mathrm{~nm}$ respectively, to obtain simulation results for the size of the current that are roughly equal to the measured data. These results suggest that the damaged $\mathrm{P} 3 \mathrm{HT}$ region in those devices is very thick. It seems unlikely that RIE can lead to such thick damaged regions. Hence, there are probably other factors that lead to a reduction of the gate effect and the size of the current. It has been reported that P3HT OFETs stored in air can be affected by water and oxygen [6]. The fabrication and storage time for the gated P3HT devices, that is, the time span between the beginning of the device fabrication and the measurements, is about five months. While this time span for the devices with $100 \mathrm{~nm}$ P3HT thickness mentioned in Chapter 5 is about two weeks. Hence, we suspect that the gated P3HT devices were probably severely affected by water and oxygen in air due to the long fabrication and storage time.

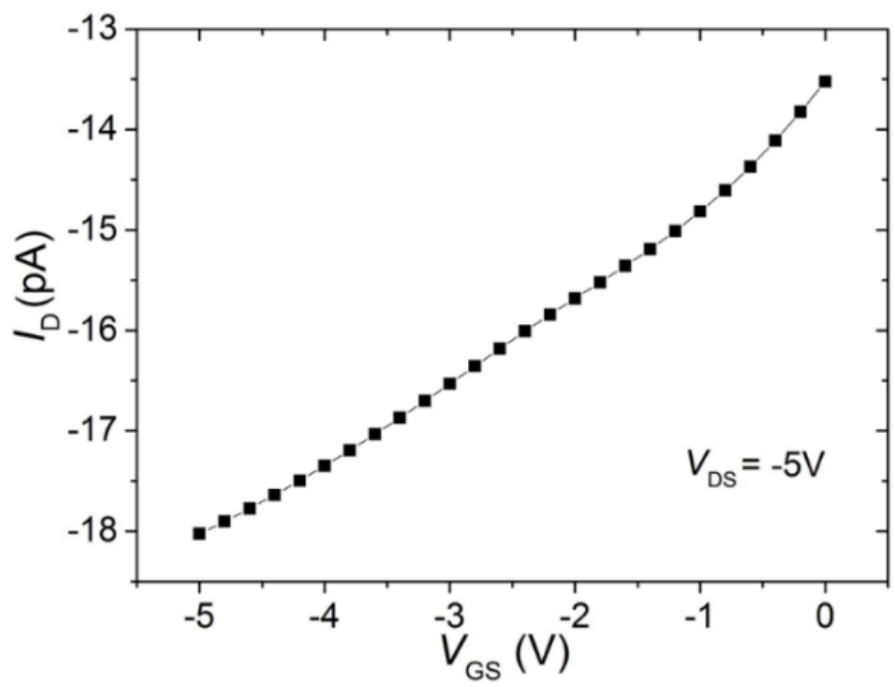

Figures 6.11: Simulated transfer characteristics of a device with $d=200 \mathrm{~nm}$ P3HT diameter, $73 \mathrm{~nm}$ gate electrode height, $25 \mathrm{~nm}$ oxide thickness and $81 \mathrm{~nm}$ damaged region thickness. 


\section{Gate electrode height dependence}

Figure 6.2 show that the full-gate device has much smaller gate electrode height than the half-gate devices. Hence, we examined the influence of the thickness of the gate electrodes on the gate effect. We simulated three devices that all had a $d=2 \mu \mathrm{m}$ P3HT diameter and a $20 \mathrm{~nm}$ oxide thickness, but three different gate electrode heights: $10 \mathrm{~nm}, 52 \mathrm{~nm}$ and 100 $\mathrm{nm}$, respectively. From Fig. 6.12 we can see that the gate effect is strengthened when the gate electrode height is increased. Hole concentration plots reveal that the stronger gate effect is caused both by a larger hole concentration around the source contact at finite gate voltage (Figs. 6.13(a) and (b)) and a stronger depletion of charge carriers around the drain contact at zero gate voltage (Figs. 6.13(c) and (d)).

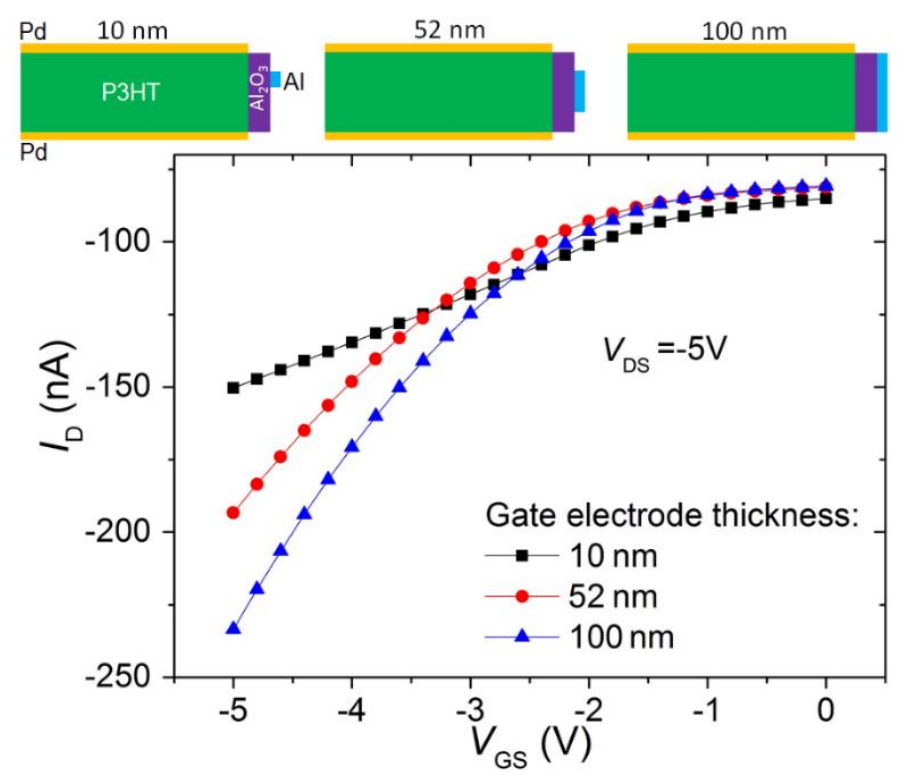

Figure 6.12: Simulated transfer characteristics of three devices. The three devices have the same P3HT diameter and oxide thickness, $2 \mu \mathrm{m}$ and $20 \mathrm{~nm}$ respectively. The only difference is the height of the gate electrodes, which is $10 \mathrm{~nm}, 52 \mathrm{~nm}$ and $100 \mathrm{~nm}$, respectively, as shown schematically in the top. 

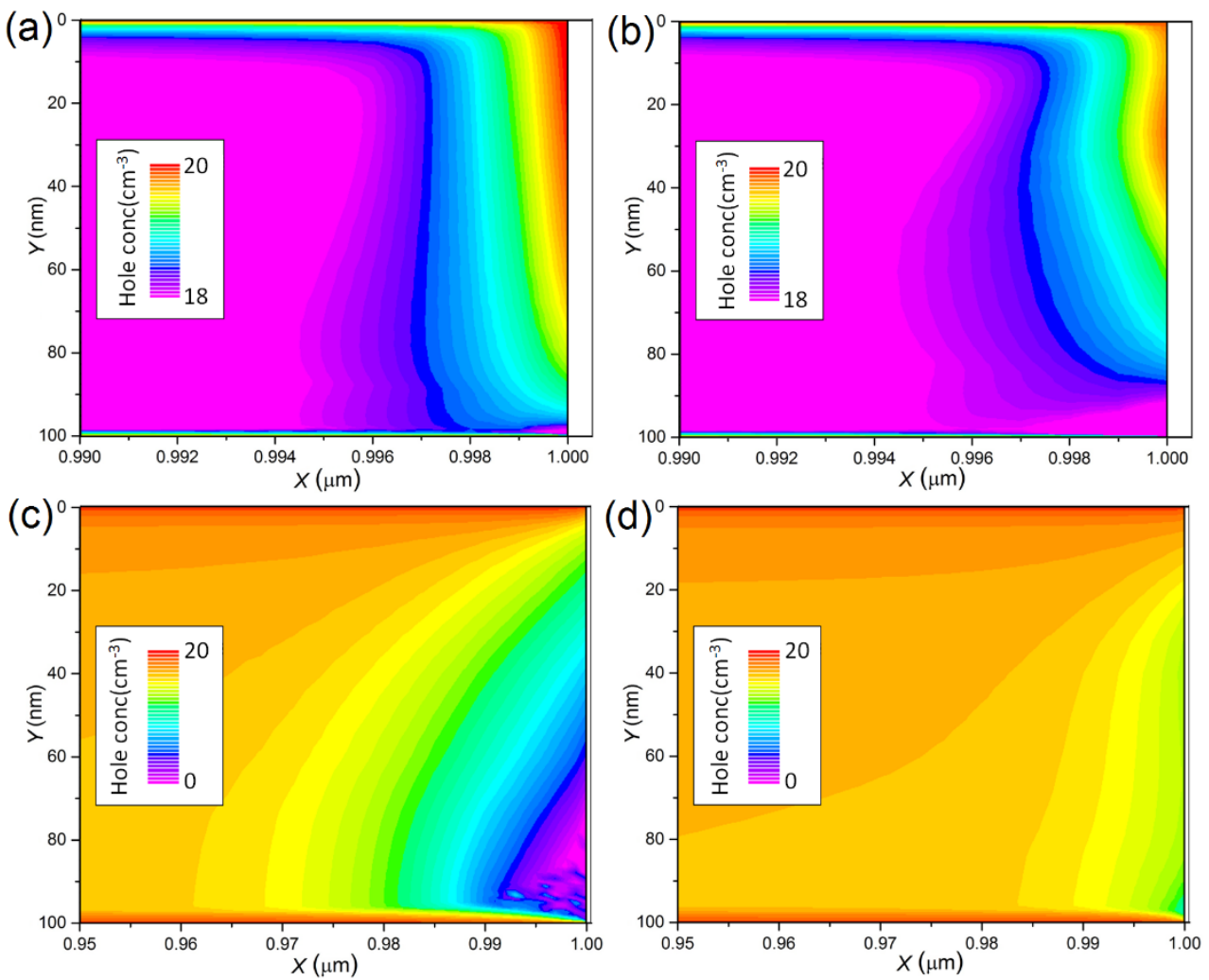

Figure 6.13: Hole concentration distributions (log scale) in two devices and a zoom-in of the region close to the organic/dielectric interface. (a) $100 \mathrm{~nm}$ high gate electrode, $V_{G S}=V_{D S}=-5 \mathrm{~V}$. (b) $10 \mathrm{~nm}$ high gate electrode, $V_{G S}=$ $V_{D S}=-5 V$. (c) $100 \mathrm{~nm}$ high gate electrode, $V_{G S}=0 V, V_{D S}=-5 V$. (d) $10 \mathrm{~nm}$ high gate electrode, $V_{G S}=0 V, V_{D S}=-5 V$.

\section{Dependence on the diameter of the P3HT pillars}

Also the influence of the diameter $d$ of the P3HT pillars on the gate effect was investigated. We found that even for a gate electrode height of $100 \mathrm{~nm}$, that is, a gate electrode fully covering the gate dielectric around the pillars, the source-drain current in the output characteristics was still not saturated in devices with a large P3HT pillar diameter (e.g., $d=$ $2 \mu \mathrm{m}$ ) when the source-drain voltage was larger than the gate voltage (Fig. 6.14(a)). As the $d$ was decreased, the source-drain current became more and more saturated for non-zero gate voltage. At zero gate voltage, the source-drain current became smaller and smaller until it was completely zero (Figs. 6.14(b)-(d)). This effect is similar to the short-channel effect in lateral OFETs [7] resulting from a decreasing channel length. The reason behind this phenomenon is the dominance of the total current by the bulk space-charge-limitedcurrent (SCLC), introduced in Chapter 2, instead of the channel conduction. It was reported 
that limiting the bulk SCLC region could suppress the short channel effect $[8,9]$, in analogy to the situation in the vertical $\mathrm{P} 3 \mathrm{HT}$ devices, where the current in the bulk region decreases relatively to that in the channel by decreasing the $\mathrm{P} 3 \mathrm{HT}$ pillar diameter. From Fig. 6.15 we can see that in the region far ( $200 \mathrm{~nm})$ away from the gate dielectric in the device with the larger $\mathrm{P} 3 \mathrm{HT}$ pillar diameter the hole concentration distribution is not affected by the gate voltage. By contrast, in the device with the smaller P3HT pillar diameter the distribution in the whole region is affected by the gate voltage.
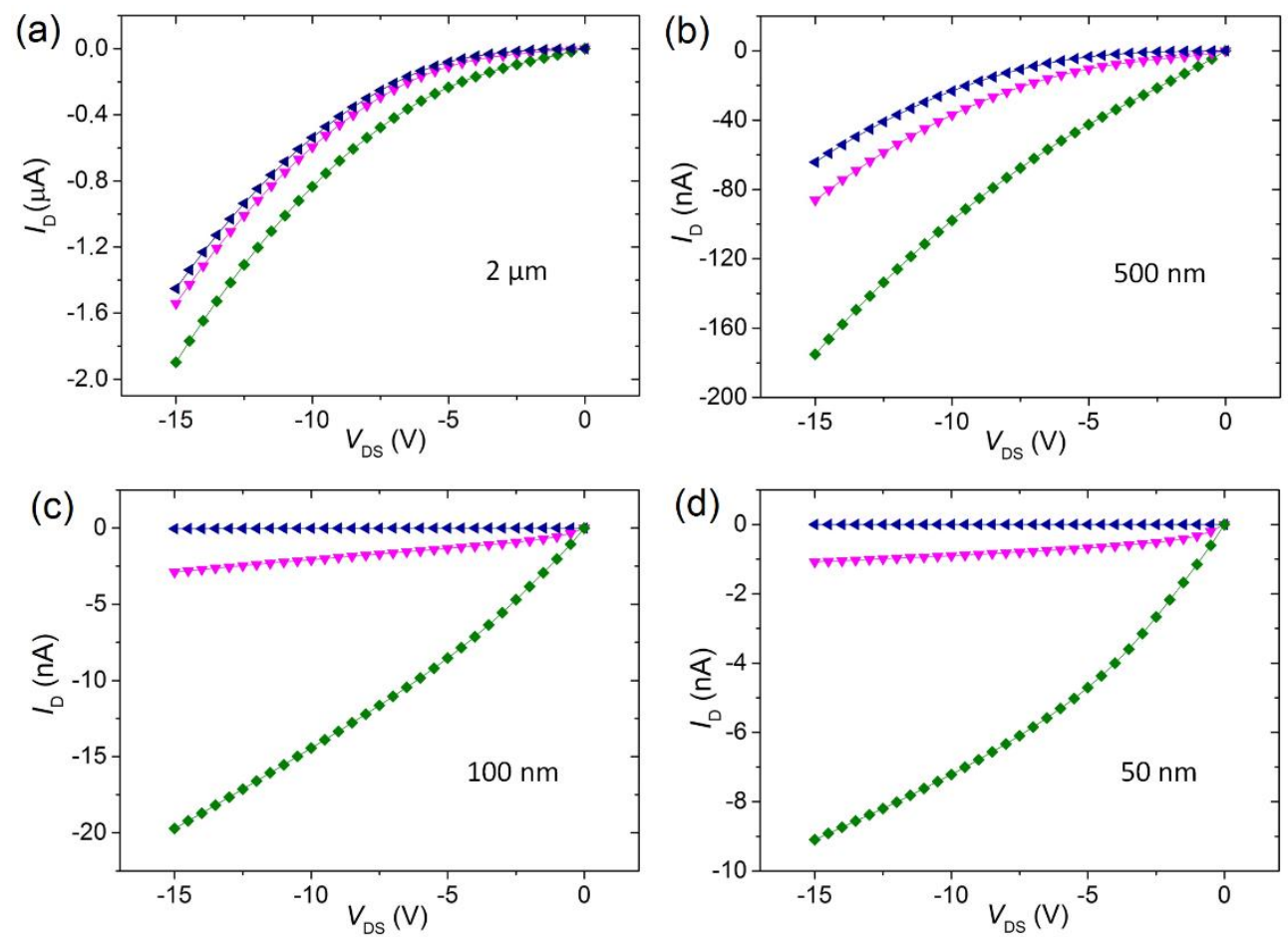

Figure 6.14: Simulated output characteristics of gated P3HT devices with four different P3HT pillar diameters d. The gate electrode height is $100 \mathrm{~nm}$ and the oxide thickness is $20 \mathrm{~nm}$. The gate voltage was fixed at three values: $0 \mathrm{~V}$ (blue left triangles), $-2.5 \mathrm{~V}$ (pink down triangles) and $-5 \mathrm{~V}$ (green diamonds). In (c), the maximum $I_{\mathrm{D}}$ at zero $V_{\mathrm{GS}}$ is about $-50 \mathrm{pA}$, while in (d) it is essentially zero (on the order of $10^{-19} \mathrm{~A}$ ). 

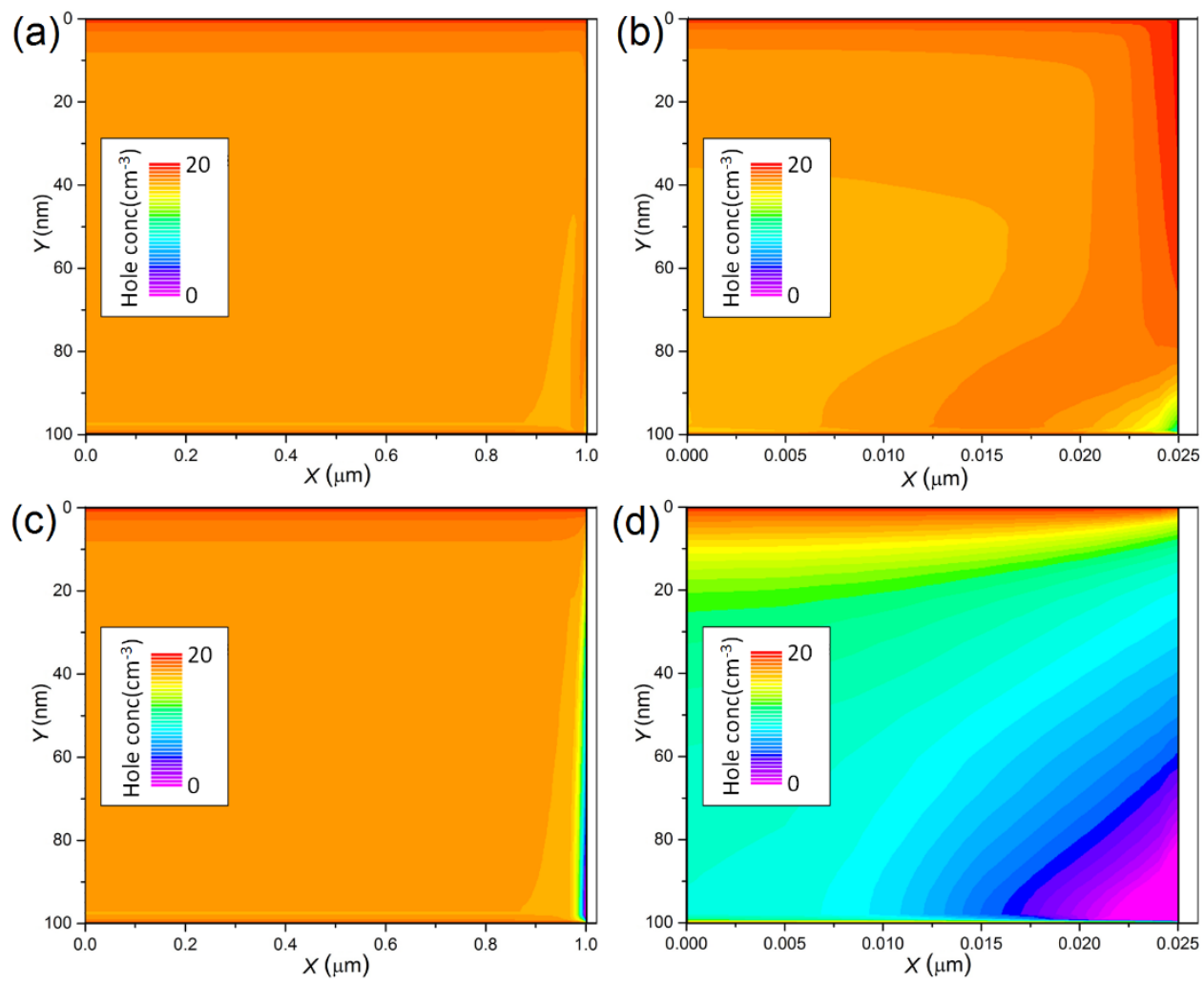

Figure 6.15: Hole concentration (log scale) in the devices corresponding to the simulation results of Fig. 6.14. (a) $d=2 \mu \mathrm{m}, V_{\mathrm{GS}}=-5 \mathrm{~V}$ and $V_{\mathrm{DS}}=-15 \mathrm{~V}$. (b) $d=50 \mathrm{~nm}, V_{\mathrm{GS}}=-5 \mathrm{~V}$ and $V_{\mathrm{DS}}=-15 \mathrm{~V}$. (c) $d=2 \mu \mathrm{m}, V_{\mathrm{GS}}=0 \mathrm{~V}$ and $V_{\mathrm{DS}}=-15 \mathrm{~V}$. (b) $d=50 \mathrm{~nm}, V_{\mathrm{GS}}=0 \mathrm{~V}$ and $V_{\mathrm{DS}}=-15 \mathrm{~V}$.

\section{Gate electrode position dependence}

Above, we concluded that the higher the gate electrode, the better the gate control. But when the gate electrode does not completely cover the gate dielectric between source and drain, would it then matter if the gate is close to the source or the drain? Device simulation results show that the gate effect is also influenced by the position of the gate electrode when the gate electrode does not completely cover the dielectric. We simulated transfer properties of devices with $10 \mathrm{~nm}$ high gate electrodes at different positions: close to the drain, close to the source, and in the middle between the drain and the source, as shown schematically in the top of Fig. 6.16. The simulated transfer properties reveal that devices with a gate in the middle have the largest transconductance (Fig. 6.16).

To understand this, we compare both the hole concentration and the hole current density distributions among the three cases. When $V_{G S}=-5 V$, devices with gate electrodes close to 
the drain have the smallest hole concentration around the source (Fig. 6.17(c)), because the gate electrodes are far away from the source. A poor accumulation layer results in a small conductivity (Fig. 6.17(f)). Devices with gate electrodes close to the source have the largest hole concentration in the accumulation layer around the source (Fig. 6.17(a)). However, their channel region is farther away from the drain than for devices with the gate in the middle (Fig. 6.17(b)). Therefore, a longer SCLC region is needed to bridge the channel region and the drain. Eventually, the total conductivity is smaller in the devices with the gate close to the source than in the devices with the gate in the middle; see the difference between Figs. 6.17(d) and (e).

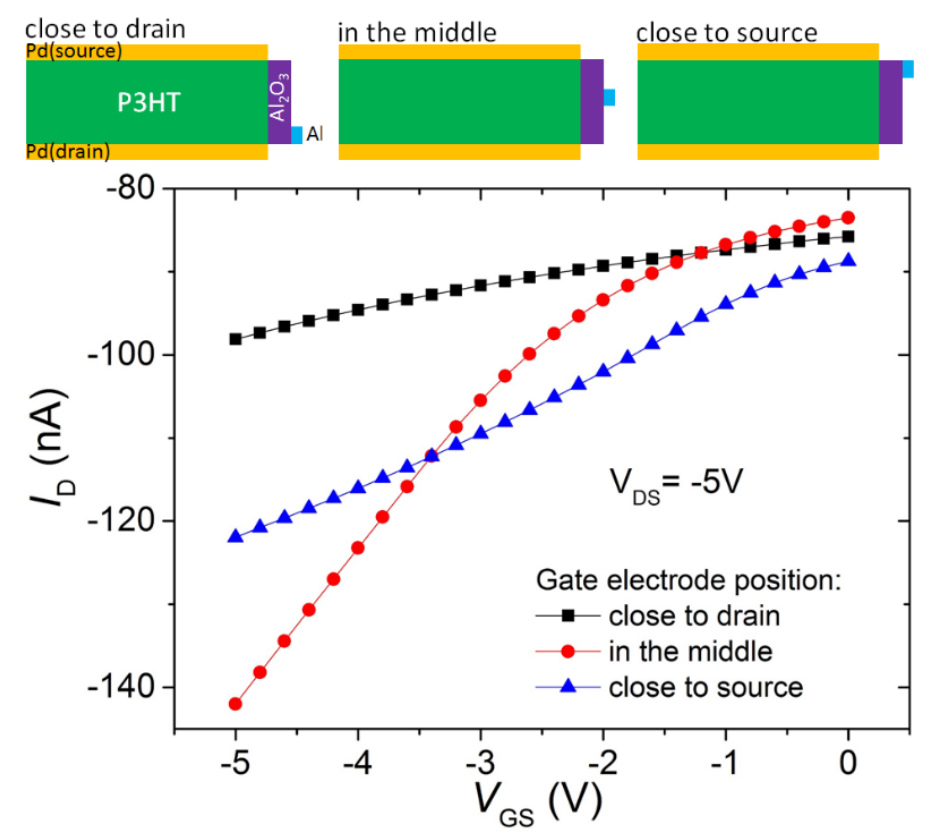

Figure 6.16: Simulated transfer characteristics of three devices with different gate electrode positions. The three devices have the same P3HT pillar diameter $d=2 \mu \mathrm{m}$, the same oxide thickness of $20 \mathrm{~nm}$, and the same gate electrode height of $10 \mathrm{~nm}$. The only difference is the position of the gate electrodes, shown schematically in the top.

Similarly, when $V_{G S}=0 \mathrm{~V}$, devices with gate close to source have the smallest depletion region around the drain (Fig. 6.18(a)). Hence, those devices show the largest conductivity (Fig. 6.18(d)). As for devices with the gate close to the drain, although they have the strongest carrier depletion around the drain, the charge carriers around the source are the least depleted, because the gate electrode is further away from the source (Fig. 6.18(c)) compared to the other two types of devices. The charge carrier conduction path between source and drain is built up as shown in Fig. 6.18(f). As for the devices with gate in the middle, 
they have a moderate depletion around both drain and source (Fig. 6.18(b)), and eventually the most suppressed conduction close to the interface (Fig. 6.18(e)).
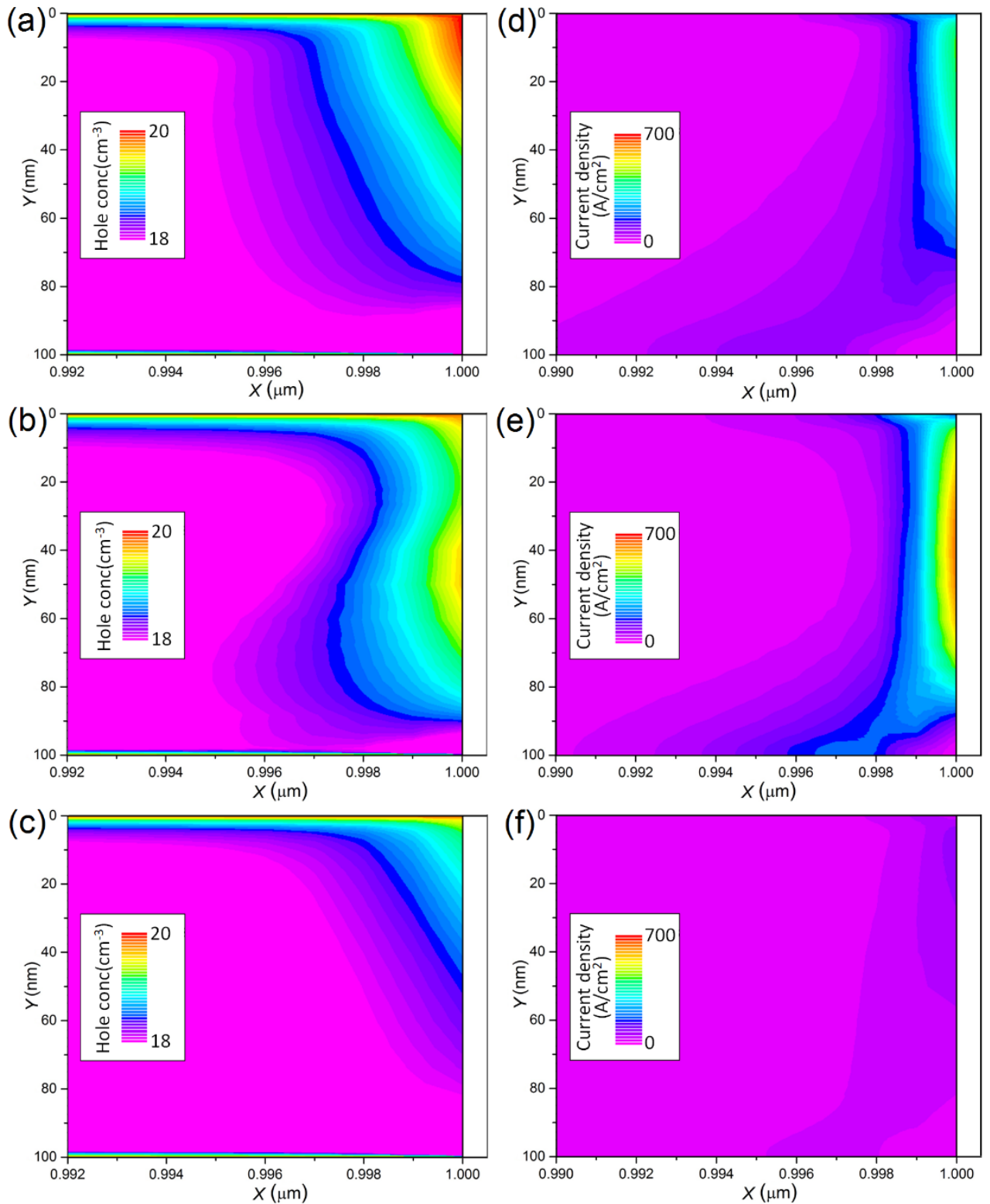

Figure 6.17: Hole concentration (log scale, (a)-(c)) and hole current density (linear scale, (d)-(f)) distribution corresponding to the results of Fig. 6.16 when $V_{G S}=-5 V$ and $V_{D S}=-5 V$. Only the region close to the oxide/dielectric interface is shown. (a) and (d): Gate close to source. (b) and (e): Gate in the middle. (c) and (f): Gate close to drain. 

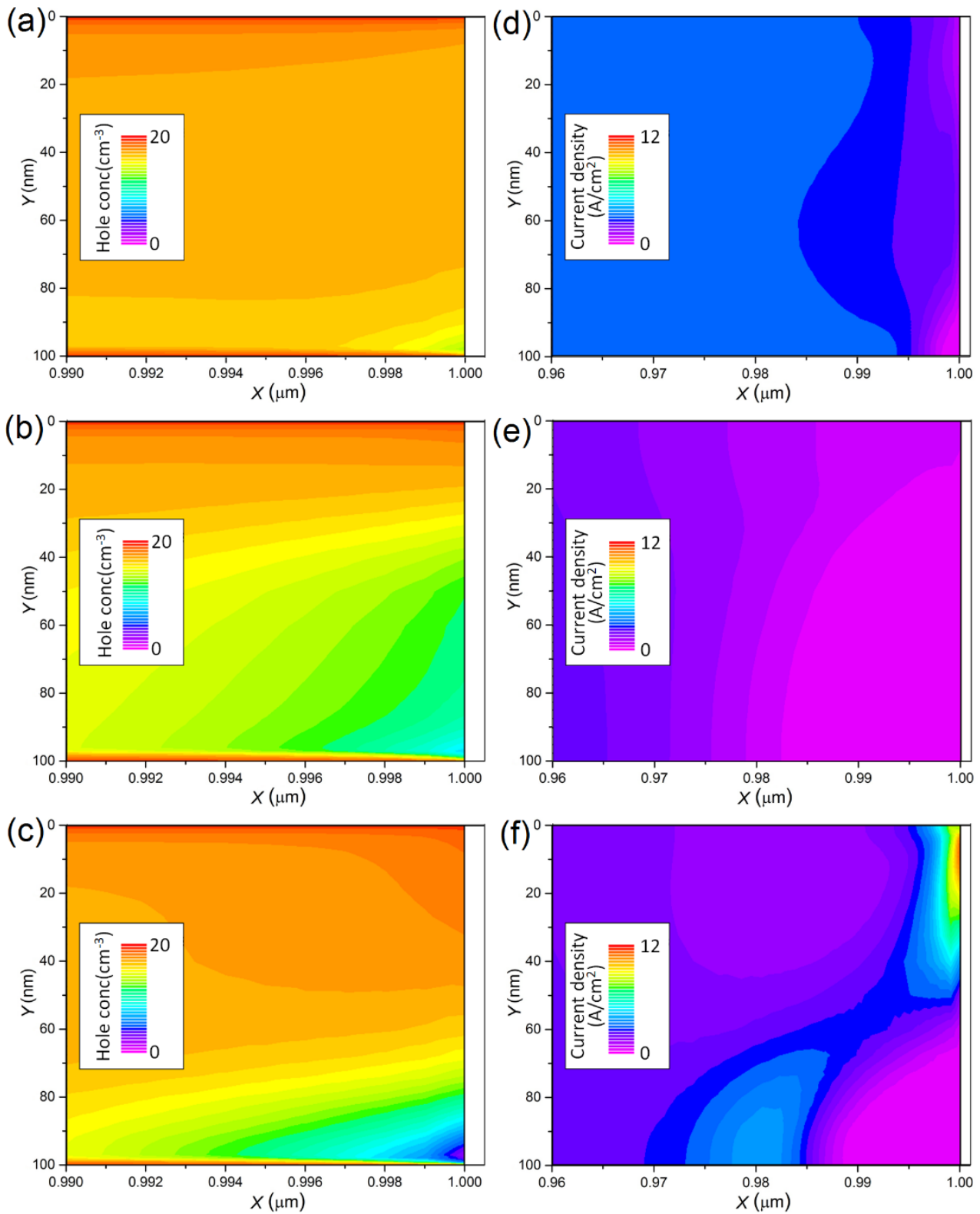

Figure 6.18: Hole concentration (log scale, (a)-(c)) and hole current density (linear scale, (d)-(f)) distribution corresponding to the results of Fig. 6.16 when $V_{G S}=0 \mathrm{~V}$ and $V_{D S}=-5 \mathrm{~V}$. Only the region close to the oxide/dielectric interface is shown. (a) and (d): Gate close to source. (b) and (e): Gate in the middle. (c) and (f): Gate close to drain. 


\section{Gate oxide thickness dependence}

We investigated the influence of the gate oxide thickness on the gate effect as well. Output characteristics of two devices with different oxide thicknesses were simulated. The devices had $d=100 \mathrm{~nm}$ P3HT pillar diameter with $100 \mathrm{~nm}$ high gate electrodes. The gate dielectric was chosen to be $10 \mathrm{~nm}$ and $20 \mathrm{~nm}$, respectively. The simulation results show that the device with a $10 \mathrm{~nm}$ thick oxide has a stronger gate effect than the other one (Fig. 6.19). This is because the gate capacitance increases when the thickness of the gate dielectric decreases, thereby strengthening the gate control.

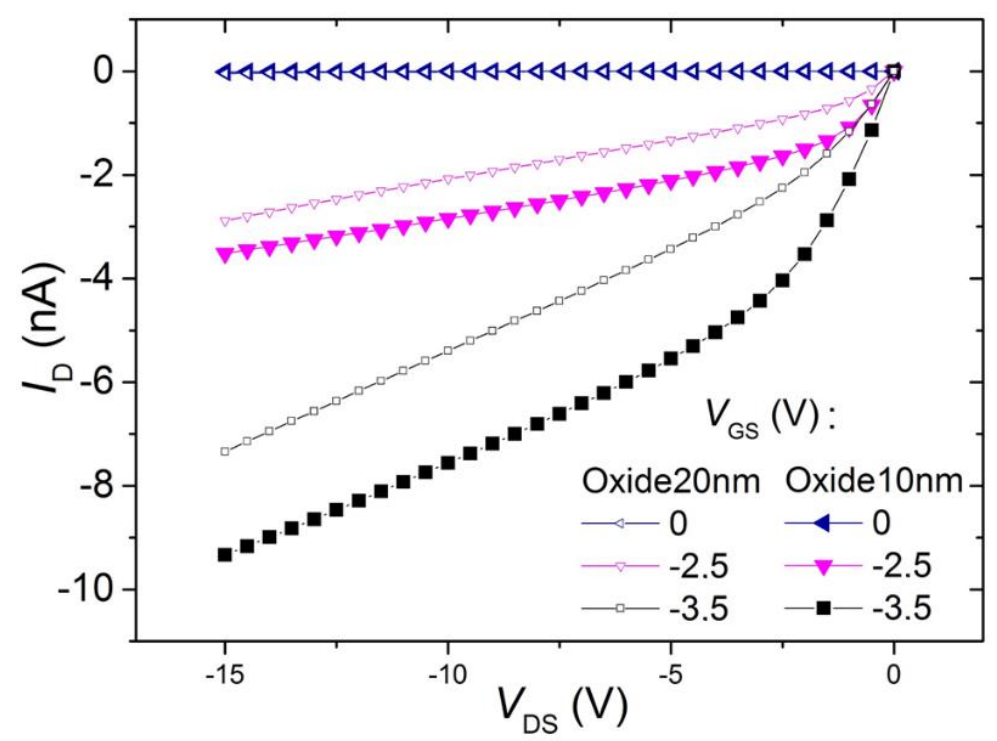

Figure 6.19: Simulated output characteristics of two devices with different gate oxide thickness: 20 and $10 \mathrm{~nm}$. The P3HT pillar diameter $d$ and the gate electrode height of the two devices are both $100 \mathrm{~nm}$.

\section{Temperature dependence of the gate effect}

Finally, the temperature dependence of the gate effect was simulated. The simulated device had a $d=2 \mu \mathrm{m}$ P3HT pillar diameter, a $10 \mathrm{~nm}$ gate electrode height and a $20 \mathrm{~nm}$ oxide thickness. Simulation results demonstrate that not only the drain current decreases with decreasing temperature, but also the transconductance (Fig. 6.20). This can be explained by the decrease of the hopping mobility when the temperature drops [10]. The temperaturedependent transport in the organic semiconductor makes a measurement of the temperature dependence a suitable way to verify whether a measured transport signal is coming from the device or from the measurement equipment. 


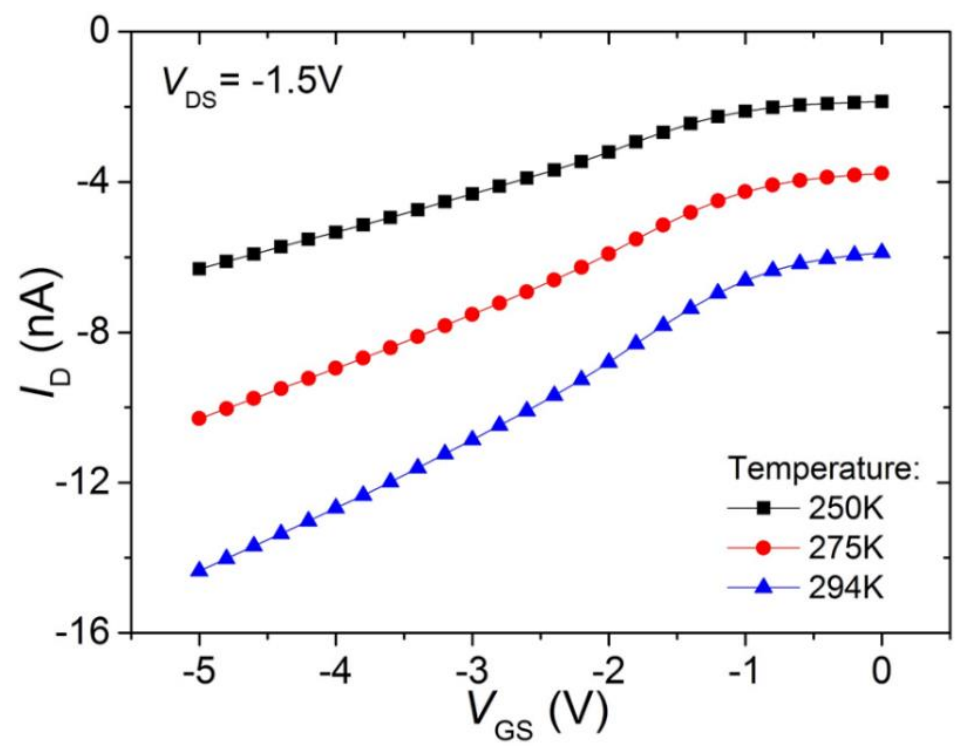

Figure 6.20: Simulated transfer characteristics of a device at three temperatures: 294, 275 and $250 \mathrm{~K}$. The device parameters are: P3HT pillar diameter $d=2 \mu \mathrm{m}, 10 \mathrm{~nm}$ gate electrode height and $20 \mathrm{~nm}$ oxide thickness.

\subsection{Summary and outlook}

We measured output and transfer properties of gated P3HT pillar devices without finding a distinct gate effect. Simulation results of ideal devices show not only a distinct gate effect, but also a larger drain current than in the experiment. Assuming a layer damaged by the RIE at the edge of the P3HT pillars, both the size of gate effect and the drain current are dramatically reduced, which suggest that a damaged layer could be a reason for the reduction of the gate effect and the conductivity. To obtain a reasonable agreement between simulated and measured results, the damaged layer has to be assumed to be very thick. Therefore, we believe that there are also other reasons for the reduction of the gate effect and the conductivity. We suspect that the devices were probably severely affected by the water and oxygen in air due to the long fabrication and storage time.

We also investigated the influence of device parameters on the gate effect. A smaller diameter of the $\mathrm{P} 3 \mathrm{HT}$ pillars, a higher gate electrode and a thinner gate dielectric all lead to better gate control. In the future, the most critical issue is to keep the organic/dielectric interface as intact as possible during the fabrication of organic field-effect transistors. In addition, regions with a large bulk current should be avoided as much as possible in the device design to enhance the gate control. 


\section{References}

1. Wilbers, J.G., Vertical hybrid inorganic-organic nanoelectronic devices. PhD thesis, Univeristy of Twente, 2016, Gildeprint.

2. ATLAS, http://www.silvaco.com/products/tcad/device_simulation/ atlas/atlas. html (Date of access: 30-12-2016)

3. Van Mensfoort, S. and R. Coehoorn, Effect of Gaussian disorder on the voltage dependence of the current density in sandwich-type devices based on organic semiconductors. Physical Review B, 2008. 78(8): p. 085207.

4. Sarkar, C.K., Technology Computer Aided Design: Simulation for VLSI MOSFET. 2013: CRC Press.

5. Cho, Y.-S. and R.R. Franklin, Conducting polymer material characterization using high frequency planar transmission line measurement. Transactions on Electrical and Electronic Materials, 2012. 13(5): p. 237-240.

6. Majewski, L. and A. Song, Extended storage time of poly (3-hexylthiophene) fieldeffect transistors via immersion in common solvents. Journal of Applied Physics, 2007. 102(7): p. 074515.

7. Haddock, J.N., et al., A comprehensive study of short channel effects in organic field-effect transistors. Organic electronics, 2006. 7(1): p. 45-54.

8. Wang, J., Z. Zheng, and H. Sirringhaus, Suppression of short-channel effects in organic thin-film transistors. Applied physics letters, 2006. 89(8): p. 083513.

9. Tukagoshi, K., et al., Suppression of short channel effect in organic thin film transistors. Applied Physics Letters, 2007. 91(11): p. 113508.

10. Li, J., et al., A stable solution-processed polymer semiconductor with record highmobility for printed transistors. Scientific reports, 2012. 2: p. 754. 


\section{Chapter 7}

\section{Conclusions and outlook}

In this chapter, we present the main conclusions of the experimental and numerical simulation results of this thesis, and present an outlook for future research based on the obtained results as well.

\section{Nanoindentation}

From the nanoindentation results reported in Chapter 3 we conclude that the nanoindentation based on the "point-and-shoot" mode of the Bruker Dimension Icon AFM (Icon AFM) is able to create holes on top of the DXP-loaded zeolite $L$ crystals with 150 $300 \mathrm{~nm}$ diameters.

In the follow-up research, devices containing DXP-loaded zeolites need to be fabricated. To realize tunneling junctions with small junction areas in LAO-STO heterostructures, the nanoindentation technique will also be used to fabricate ultrasmall ( $10 \mathrm{~nm})$ metallic contacts on top of LAO-STO heterostructures. With small junction areas, suppression of structural fluctuations is expected to be suppressed. This nanoindentation method also offers a good option to fabricate metallic nanocontacts on other submicron objects, such as organic single-crystals (see the DXP crystals in Fig. 4.1(a)) which are self-organized on conducting substrates. Therefore, organic single-crystal junctions can be easily fabricated.

\section{DXP lateral field-effect transistors}

The DXP lateral field-effect transistors exhibit $n$-type channel behavior based on the output and transfer characteristics.

However, there are also some issues to be addressed, such as the non-saturated output characteristics, large hysteresis in the transfer characteristics. Next, further experiments are necessary to confirm and improve the channel behavior. For the device fabrication, thermal evaporation and substrate heating during deposition of DXP are highly preferable to achieve continuous uniform films with high crystallinity to improve the conductivity. This might also suppress the hysteresis in both the output and transfer characteristics. In addition, HMDS/OTS treatment of the $\mathrm{SiO}_{2}$ surface is promising for decreasing the hysteresis in the 
transfer characteristics. To verify the short-channel effect in the lateral DXP devices, we need to investigate systematically both long-channel and short-channel devices. Organic magnetoresistance (OMAR) in organic field-effect transistors has been reported [1, 2]. We recommend to measure the magnetic-field dependence of electrical transport in DXP fieldeffect transistors.

\section{Vertical P3HT pillar devices}

Two-terminal devices. The fabrication method described in Chapter 5 can be used to realize vertical $\mathrm{P} 3 \mathrm{HT}$ devices with sub-100 nm junction length and a well-defined interface between the metallic contact and the P3HT layer. More significantly, the devices are robust under high current densities and reveal thermally assisted hopping transport. The current density in the vertical P3HT device with an ultrashort junction length $(5 \mathrm{~nm})$ reaches $10^{6} \mathrm{~A} / \mathrm{m}^{2}$, which is much larger than the values obtained in other P3HT devices [3][4]. To our knowledge, such high current densities have not been reported so far. The high current density is beneficial to many applications, such as organic light-emitting transistors and display pixel drivers [5]. Simulation results based on the drift-diffusion model with a Gaussian density of states indicate a low injection barrier (less than $0.1 \mathrm{eV}$ ), revealing very good hole injection.

The fabrication technique yields the prospect of new types of very thin and highly conducting organic devices. In the future, more organic materials could be characterized using this fabrication technique. Apart from the organic layer, it is also possible to use different electrode materials, such as ferromagnetic or superconductive materials. Therefore, more physics experiments could be performed.

Vertical P3HT field-effect transistors. With the fabrication method described in Chapter 6 we can realize gate dielectric layers and surrounding gate electrodes around the twoterminal vertical P3HT junctions. Although a distinct gate effect is revealed in the ATLAS device simulation results, the measured output and transfer characteristics of the devices do not show a clear gate effect. The simulated conductivity is also larger than the measured one. We propose that a layer damaged by the dry etching at the edge of the P3HT pillars could be the reason for the reduction of the gate effect and the conductivity, which is supported by the device simulation results. From the simulation results of ideal devices, we also learn that a smaller diameter of the P3HT pillars, a larger overlap of the gate dielectric and the gate electrode around the pillars, and a thinner gate dielectric all lead to better gate control. 
The ATLAS device simulation results remind us of the importance of the organic/dielectric interface in OFETs. Keeping the interface as intact as possible should be taken into account during the fabrication of OFETs. In addition, regions with a large bulk current should be avoided as much as possible in the device design to enhance the gate control.

\section{References}

1. Reichert, T. and T.P. Saragi, Photoinduced magnetoresistance in organic field-effect transistors. Applied physics letters, 2011. 98(6): p. 29.

2. Isenberg, C. and T.P. Saragi, Revealing the origin of magnetoresistance in unipolar amorphous organic field-effect transistors. Journal of Materials Chemistry C, 2014. 2(40): p. 8569-8577.

3. Sirringhaus, H., N. Tessler, and R.H. Friend, Integrated optoelectronic devices based on conjugated polymers. Science, 1998. 280(5370): p. 1741-1744.

4. Tanase, C., et al., Unification of the hole transport in polymeric field-effect transistors and light-emitting diodes. Physical Review Letters, 2003. 91(21): p. 216601.

5. Wilbers, J.G.E., et al., Charge transport in nanoscale vertical organic semiconductor pillar devices. Scientific Reports, 2017. 7: p. 41171. 


\section{Summary}

Organic semiconductors have been drawing more and more attention due to their huge potential for low-cost, flexible, printable electronics and spintronics. N, N'-bis(2,6dimethylphenyl)-perylene-3,4,9,10-tetracarboxylic diimide (DXP) is an organic semiconductor. DXP-loaded zeolite $L$ crystals is expected to have unique spin-dependent transport property due to the strictly one-dimensional constrain. To realize various electrical transport measurements on the DXP-loaded zeolite L crystals, we design a fabrication method based on nanoindentation to embed the crystals into devices. Besides the DXP-loaded zeolite L crystals, we also plan to explore the spin-dependent transport property of the DXP thin films. Hence, we fabricate and investigate DXP lateral field-effect transistors. For an organic semiconductor device, short junction/channel length is beneficial to scaling down the integrated circuits based on the organic semiconductor device, high cutoff frequency, low operation voltage, and so on. In lateral devices, nanolithography techniques are usually necessary to fabricate short spacing electrodes. However, in a vertical configuration where an organic film is sandwiched between two metallic contacts, the channel length is defined by the film thickness which is well controllable down to a few $\mathrm{nm}$. Nevertheless, fabricating top contacts on thin organic films and patterning organic layers are not straightforward. So, we demonstrate a fabrication method to realize vertical organic devices with short junction/channel length. Poly(3-hexylthiophene) (P3HT) is a widely-investigated organic semiconductor. It has good electrical conductivity. It is a polymeric material and can form continuous and flat thin films by spin-coating. Hence, we fabricate vertical P3HT devices and investigate the charge transport of the devices.

We start in Chapter 1 by putting the experimental and numerical results in context and provide a motivation for our work.

Chapter 2 concisely discusses the theoretical background related to the experimental and numerical simulation research reported in this thesis.

In Chapter 3, a fabrication method based on nanoindentation using atomic force microscope (AFM) is introduced for electron transport measurements on one-dimensional DXP-loaded zeolite $\mathrm{L}$ crystals and two-dimensional electron systems in $\mathrm{LaAlO}_{3} / \mathrm{SrTiO}_{3}$ heterostructures. Parylene- $\mathrm{C}$ is used as the indent material. Three common methods using the Veeco Dimension 3100 AFM (Veeco AFM), and the so-called "point-and-shoot" mode of the Bruker Dimension Icon AFM (Icon AFM) have been used to perform nanoindentation on DXP-loaded zeolite $L$ crystals with $\sim 150-300 \mathrm{~nm}$ diameter. The nanoindentation results demonstrate that the common methods do not meet the positioning requirement. Nanoindentation using the "point-and-shoot" function is able to create indents on top of the sub-micron zeolites. Au-parylene-Au test devices have been fabricated using the fabrication method. IV measurements of the test devices with the nanoindentation show high conduction (about $50 \Omega$ ). Test devices without nanoindentation show good insulation (about $70 \mathrm{G} \Omega$ ). The $I V$ measurement results demonstrate that the fabrication method based 
on the nanoindentation using the "point-and-shoot" mode of the Icon AFM is suitable for the fabrication of nanocontacts on the DXP-loaded zeolites and $\mathrm{LaAlO}_{3} / \mathrm{SrTiO}_{3}$ samples.

Chapter 4 discusses the results on DXP lateral field-effect transistors. For the final purpose of investigating magnetic field effects in the electrical transport properties of DXP, DXP lateral field-effect transistors have been fabricated by drop-casting DXP onto interdigitated $\mathrm{Au}$ electrodes pre-fabricated on $\mathrm{SiO}_{2} / \mathrm{Si}$ substrates. Electro-migration of the electrodes after IV measurements has also been observed. The electrical transport property of the DXP devices are light sensitive and much more stable when measuring in vacuum ( $10^{-5} \mathrm{mbar}$ ) than in ambient atmosphere. Based on the output and transfer characteristics, the DXP devices exhibit n-type channel behavior. The output characteristics do not show saturation, and the threshold voltage of the transfer characteristics is drain-bias dependent. We propose that this is probably due to the short-channel effect. Large hysteresis in the transfer characteristics is also observed. We ascribe this to the bias stress effect caused by the untreated organic/dielectric interface. Further research is needed to confirm and improve the channel behavior of the DXP lateral devices. Spin-dependent transport measurements will also be executed.

In Chapter 5, two-terminal vertical P3HT pillar devices have been fabricated by wedging transfer and utilizing the wedge-transferred Au top contacts as etch masks for directional dry reactive ion etching (RIE) of the P3HT thin film. The diameters of the P3HT pillars are 2 $\mu \mathrm{m}$ to $200 \mathrm{~nm}$ and the thicknesses of the P3HT layers are $5 \mathrm{~nm}$ to $100 \mathrm{~nm}$. SEM images show that the $\mathrm{P} 3 \mathrm{HT}$ is well protected by the top contacts, and that there is a distinctive interface between the metal and the organic layer, suggesting that the metal does not penetrate the $\mathrm{P} 3 \mathrm{HT}$. The devices exhibit good electrical reproducibility when measuring in vacuum $\left(<10^{-4}\right.$ mbar). The relatively high working device yield of $40-85 \%$ indicates that the top-contacting is very soft, allowing for charge transport through very thin organic films. The $5 \mathrm{~nm}$ thick $\mathrm{P} 3 \mathrm{HT}$ junctions carry very high current density, up to $10^{6} \mathrm{~A} / \mathrm{m}^{2}$. The temperature dependence of the electrical transport has been measured between room temperature and $150 \mathrm{~K}$. The results reveal thermally assisted hopping transport.

Simulation of the temperature dependence has been performed based on the driftdiffusion model with a Gaussian density of states. Experimental results of the devices with P3HT thicknesses of 40 and $100 \mathrm{~nm}$ can be explained by the model very well. The simulated results indicate a low injection barrier (less than $0.1 \mathrm{eV}$ ), which can explain the weaker temperature dependence of the devices with thinner P3HT. However, for devices with P3HT thicknesses of 5 and $10 \mathrm{~nm}$, the simulated current densities for the P3HT thicknesses of 10 $\mathrm{nm}$ and $5 \mathrm{~nm}$ are higher than the measured values. Nonetheless, the measured temperature dependence is still well described by the simulations. We attribute the discrepancy between experiment and simulations to a different orientation of the P3HT chains close to the $\mathrm{Au}$ electrodes as compared to the bulk. We conclude that carrier injection in our devices is very good, yielding the prospect of new types of very thin and highly conducting organic devices.

Follow up on this work, we have investigated gated vertical P3HT pillar devices in Chapter 6. Output and transfer properties of the gated devices have been measured without finding a distinct gate effect. Device simulations have been performed as well, using the 
commercial software package Silvaco ATLAS. Simulation results of ideal devices show not only a distinct gate effect, but also a larger drain current than in the experiment. Assuming a damaged layer at the edge of the P3HT pillars due to the RIE, both the gate effect and the drain current are dramatically reduced. This suggests that a damaged layer could be the reason for the reduction of the gate effect and conductivity. To obtain reasonable agreement between simulated and measured results, the damaged layer has to be assumed very thick. Therefore, we believe that there are also other reasons for the small gate effect and conductivity. We suspect that the devices were probably severely affected by the oxygen and water in the air due to the long fabrication and storage time before the electrical characterization. The influence of several device parameters on the gate effect has also been investigated. A smaller P3HT pillar diameter, a larger overlap of the gate dielectric and the gate electrode around the pillars, and a thinner gate dielectric all lead to better gate control. In the future, the most critical issue is to keep the organic/dielectric interface as intact as possible during the fabrication of organic field-effect transistors. In addition, regions with a large bulk current should be avoided as much as possible in the device design to enhance the gate control.

In Chapter 7 we provide a general discussion of the experimental and numerical results obtained in this thesis, and give an outlook for future research. 


\section{Samenvatting}

Organische halfgeleiders staan meer en meer in de belangstelling dankzij het veelbelovende voortuitzicht van goedkope, flexibele en printbare elektronica en spintronica. N,N'-bis(2,6dimethylfenyl)-peryleen-3,4,9,10-tetracarboxyl diimide (DXP) is een organische halfgeleider. Men verwacht dat met DXP gevulde zeoliet-L-kristallen unieke spinafhankelijke transporteigenschappen hebben dankzij de strikt-eendimensionale opsluiting. Om verscheidene transportmetingen te kunnen uitvoeren op de met DXP gevulde zeolietL-kristallen hebben we een fabricagemethode, gebaseerd op nano-inkerving, ontwikkeld om de kristallen in devices te kunnen opnemen. Naast de met DXP gevulde zeoliet-Lkristallen zijn we ook van plan om de spin-afhankelijke transporteigenschappen van dunne DXP lagen te onderzoeken. Voor dat doel hebben we laterale veldeffecttransistoren met DXP gemaakt en gemeten. Voor een device met een organische halfgeleider is een korte junctie-/kanaallengte voordelig voor de schaalbaarheid van geïntegreerde schakelingen gebaseerd op het organische-halfgeleiderdevice, en voor het verkrijgen van, onder meer, een hoge kantelfrequentie en een lage werkspanning. In laterale devices zijn nanolithografie-methodes noodzakelijk om elektrodes op korte afstand van elkaar te maken. In een verticale structuur, waar een organische laag ingeklemd zit tussen twee metallische elektrodes, wordt, daarentegen, de kanaallengte bepaald door de dikte van de laag, die goed controleerbaar is tot op enkele $\mathrm{nm}$. Niettemin zijn het maken van topelektrodes op dunne organische lagen en het creëren van patronen in organische lagen alles behalve eenvoudig. Daarom presenteren wij een methode voor de fabricage van verticale, organische devices met korte junctie-/kanaallengtes. Poly(3-hexylthiofeen) (P3HT) is een breed-onderzochte, organische halfgeleider met goede elektrische geleiding. Het is een polymeer materiaal waarvan, met behulp van spincoating, dunne, vlakke lagen gemaakt kunnen worden. Om die reden maken we verticale P3HT-devices en onderzoeken het ladingstransport van deze devices.

We beginnen in Hoofdstuk 1 met het in perspectief plaatsen van de bereikte experimentele en numerieke resultaten. Tevens geven we een motivatie voor ons werk.

Hoofdstuk 2 bevat een beknopt overzicht van de theoretische achtergronden behorend bij het experimentele en numeriek-gesimuleerde onderzoek dat gepresenteerd wordt in dit proefschrift.

In Hoofdstuk 3 introduceren we een fabricagemethode gebaseerd op nano-inkerving met een atoomkrachtmicroscoop (AFM) voor metingen van het elektrische transport in 
eendimensionale, met DXP gevulde zeoliet-L-kristallen en tweedimensionale elektronensystemen in $\mathrm{LaAlO}_{3} / \mathrm{SrTiO}_{3}$-heterostructuren. Paryleen-C wordt gebruikt als het in te kerven materiaal. Drie standaard modi van de Veeco Dimension 3100 AFM (Veeco AFM), en de zogeheten "point-and-shoot"-modus van de Bruker Dimension Icon AFM (Icon AFM) zijn gebruikt voor het maken van nano-inkervingen op met DXP gevulde zeoliet-Lkristallen met diameters van ongeveer 150 tot $300 \mathrm{~nm}$. De resultaten van het nano-inkerven laten zien dat de standaard modi niet voldoen wat betreft de precisie waarmee de inkerving kan worden gepositioneerd. Met behulp van de "point-and-shoot"-modus is het mogelijk nano-inkervingen te maken bovenop de sub-micron zeolieten. Au-paryleen-Au-testdevices zijn met deze fabricagemethode gemaakt. IV-metingen aan deze testdevices met nanoinkervingen laten hoge geleiding (ongeveer $50 \Omega$ ) zien. Testdevices zonder nanoinkervingen vertonen juist goede elektrisch isolatie (rond $70 \mathrm{G} \Omega$ ). De IV-metingen laten zien dat de gebruikte fabricagemethode met nano-inkerving via de "point-and-shoot"-modus van de Icon AFM geschikt is voor het maken van nano-contacten op de met DXP gevulde zeolieten en de $\mathrm{LaAlO}_{3} / \mathrm{SrTiO}_{3}$-structuren.

Hoofdstuk 4 bespreekt de resultaten van laterale veldeffecttransistoren met DXP. Voor het uiteindelijke doel om de invloed van magnetische velden op de elektrische transporteigenschappen van DXP te onderzoeken, zijn laterale veldeffecttransistoren gemaakt door DXP te druppelgieten op vooraf gefabriceerde, in elkaar grijpende Auelektrodes op $\mathrm{SiO}_{2} / \mathrm{Si}$-substraten. Na het uitvoeren van IV-metingen is elektromigratie van de elektrodes waargenomen. De elektrische transporteigenschappen van de DXP-devices zijn lichtgevoelig en veel stabieler wanneer zij worden gemeten in een vacuüm ( $10^{-5} \mathrm{mbar}$ ) dan onder atmosferische druk. Gebaseerd op de output- en overdrachtskarateristieken vertoont het DXP n-type gedrag. De output-karakteristieken laten geen verzadiging zien en de omslagspanning is afhankelijk van de aangelegde drain-spanning. We stellen dat dit waarschijnlijk wordt veroorzaakt door de korte kanaallengte. Tevens zien we sterke hysterese inde overdrachtskarakteristieken. Dit schrijven we toe aan het bias-stresseffect veroorzaakt door het onbehandelde grensvlak tussen het organische materiaal en het diëlektricum. Verder onderzoek is nodig om het geleidingsgedrag van de laterale DXPdevices te bevestigen en verbeteren. Ook zullen er spin-afhankelijke transportmetingen worden gedaan.

In Hoofdstuk 5 zijn verticale, pilaarvormige P3HT-devices met twee elektrodes gemaakt door middel van "wedging transfer", waarna de aangebrachte Au topelektrodes als etsmasker zijn gebruikt voor het droogetsen van de P3HT-laag. De diameters van de P3HTpilaren variëren van $2 \mu \mathrm{m}$ tot 200 nmen de diktes van de P3HT-lagen liggen tussen de $5 \mathrm{~nm}$ en $100 \mathrm{~nm}$. SEM-opnames laten zien dat het P3HT goed beschermd wordt door de 
topelektrodes. Ook is er een duidelijk waarneembaar grensvlak tussen het metaal en de organische laag, wat suggereert dat het metaal niet doordringt in de P3HT-laag. De devices vertonen goed-reproduceerbaar elektrisch gedrag bij metingen in vacuüm $\left(<10^{-4} \mathrm{mbar}\right)$. De relatief hoge opbrengst van werkende device van 40-85\% laat zien dat er met de gebruikte methode heel zacht contact gemaakt wordt, wat ladingstransport door zeer dunne organische lagen mogelijk maakt. De $5 \mathrm{~nm}$ dikke P3HT-juncties dragen een zeer hoge stroomdichtheid, tot $10^{6} \mathrm{~A} / \mathrm{m}^{2}$. De temperatuursafhankelijkheid van het elektrisch transport is gemeten in het bereik van kamertemperatuur tot $150 \mathrm{~K}$. De resultaten laten thermisch ondersteund hopping-transport zien.

Simulaties van de temperatuursafhankelijkheid zijn uitgevoerd gebaseerd op het driftdiffusiemodel met een Gaussische toestandsdichtheid. Experimentele resultaten van de devices met P3HT-diktes van 40 en 100 nm kunnen zeer goed worden verklaard met behulp van dit model. De simulaties laten een lage injectiebarrière (minder dan $0.1 \mathrm{eV}$ ) zien, wat de zwakkere temperatuursafhankelijkheid van de devices met dunnere P3HT-lagen kan verklaren. Voor de devices met P3HT-diktes van 5 en $10 \mathrm{~nm}$ zijn de gesimuleerde stroomdichtheden echter hoger dan de gemeten waardes. Desalniettemin wordt de gemeten temperatuursafhankelijkheid goed beschreven door de simulaties. Verschillen tussen experiment en simulaties dichten we toe aan een afwijkende oriëntatie van de P3HTketens vlakbij de Au-elektroden in vergelijking met de rest van het materiaal. We concluderen dat de injectie van ladingsdragers erg goed is in onze devices, veelbelovend voor de realisatie van nieuwe soorten zeer dunne en zeer goed geleidende organische devices.

Voortbouwend op dit werk hebben in Hoofdstuk 6 verticale, pilaarvormige P3HT-devices met gates onderzocht. Metingen van output- en overdrachtskarakteristieken van deze devices laten geen uitgesproken gate-effect zien. Daarnaast zijn simulaties van deze devices uitgevoerd met behulp van het commerciële softwarepakket Silvaco ATLAS. Gesimuleerde, ideale devices laten niet alleen een duidelijk gate-effect zien, maar vertonen ook hogere stroomsterktes door de drain dan experimenteel waargenomen. De aanwezigheid van een beschadigd gebied aan de rand van de P3HT pilaren leidt tot een sterke afname van het gate-effect en de drain-stroomsterkte in de gesimuleerde devices. Dit maakt het waarschijnlijk dat de gemeten lagere gate-effect en geleiding het resultaat zijn van beschadigingen veroorzaakt door het RIE-proces. Om redelijke overeenstemming te verkrijgen tussen de gesimuleerde en gemeten resultaten zou deze beschadigde laag echter erg dik moeten zijn. We verwachten daarom dat er nog andere oorzaken zijn die bijdragen aan de verschillen. We vermoeden dat langdurige blootstelling aan in de lucht aanwezig zuurstof en water gedurende zowel de fabricage als de lange opslagtijd voordat de 
elektrische transportmetingen zijn uitgevoerd de devices sterk heeft beïnvloed. Ook is de invloed van enkele device-eigenschappen op het gate-effect onderzocht. Een kleinere diameter van de $\mathrm{P} 3 \mathrm{HT}$ pilaar, een grotere overlap van het gate-diëlektricum met de gateelektrode rondom de pilaren, en een dunner gate-diëlektricum leiden allemaal tot betere gate-controle. Voor toekomstige toepassingen is het van kritiek belang dat het grensvlak tussen het organische materiaal en het diëlektricum onbeschadigd blijft tijdens de fabricage van organische veldeffecttransistoren. Daarnaast moeten gebieden met een hoge bulk stroom zoveel mogelijk worden vermeden in het device-ontwerp om de gate-controle te verbeteren.

In Hoofdstuk 7 geven we een algemene discussie van de experimentele en numerieke resultaten van dit proefschrift, en blikken we vooruit naar toekomstig onderzoek. 


\section{Acknowledgements}

Whilst writing this part of my thesis, I realize that not just the manuscript is ending but also a stage of my life is coming to an end. Life is not easy, especially doing a PhD and without many peoples' company and help, I could not have proceeded. Hereby, I would like to express my sincere gratitude to my dear colleagues, friends and family; thank you!

First of all, I would like to thank Wilfred for offering me this PhD position and supervising me for the last four years. I learned a lot from your extensive knowledge. You also enlightened me time after time on carefully handling details in experiments, efficiently and purposively arranging work, properly writing a scientific report and performing an academic presentation, and so on. As a group leader, you have been encouraging a harmonic and socially active atmosphere within the NanoElectronics group, which makes me feel like being at home.

I appreciate very much that Prof. Paul W. M. Blom, Prof. Bart Jan Ravoo, Prof. Gertjan Koster, Prof. Peter A. Bobbert, and Dr. Michel P. de Jong agreed to be the graduation committee members of my PhD defense. Thank you very much for evaluating my concept thesis and giving valuable comments. Peter, it was a great pleasure to work with you. You illuminated many physical concepts and theories to me. Michel, thank you for being so friendly and helpful. You have extensive theoretical and experimental knowledge. It is always inspiring to discuss with you.

It is a great pleasure to be an element of the NanoElectronics group. Thank you, all group members, for creating such a pleasant environment, thereby bringing me a wonderful PhD time decorated with those enjoyable NEvents, journal clubs, NE cake-moments. I would like to specially thank some group members. Martin, you are really an electronics and SPM master. Thank you very much for explaining to me a lot about AFM and assisting me with the electrical transport measurements. Thijs, thanks a lot for the help with the experiments, the computer and ICT account issues, especially for building the superior LabVIEW programs. Johnny (Sanderink), thank you for the cleanroom work you did for me. Karen and Susan, thanks to you, I had never been troubled by the administrative work. I would like to thank my office mates, Dilu, Frank, Tamer, Celestine and Kai, for building such a nice office ambience. Kai, thanks a lot for the help with the low-temperature and Bruker experiments. Celestine and Frank, thank you for sparing time to be my paranymphs. Janine and Tamer, it was enormously fortunate to share the same projects with you. Owing to your splendid work, my PhD could progress smoothly. Janine, I am very glad that our work has already 
been published in Scientific Reports. Elmer, you are always very kind. Thank you very much for the excellent Dutch translation of the summary of this thesis. Sergey and Derya, many thanks for growing the $\mathrm{SiO}_{2}$ wafers for me.

My PhD research considerably depended on the nanofabrication work in the cleanroom of the MESA+ Institute for Nanotechnology. Hence, I would like to thank all cleanroom staff members for instructing me how to use the equipment and maintaining normal operation of the whole cleanroom. During my PhD, I also received plenty of help from other research groups at the University of Twente. The nanoindentation work described in this thesis was performed using the ICON AFM of the Physics of complex fluids (PCF) group. I also used the experimental equipment in the chemical labs of the Molecular nanofabrication (MNF) group many times in the last four years. Besides, I also used the PPMS of the Inorganic materials science (IMS) group. The device simulations reported in this thesis were performed using the ATLAS software of the Semiconductor components (SC) group. So, thank you all for supporting my work. Furthermore, I would like to specially thank Dr. Ray Hueting of the SC group for instructing me in the ATLAS software. Without your valuable help, Chapter 6 of this thesis would not exist. In addition, I also would like to specially thank Prof. Luisa De Cola and Dr. Nadia Licciardello of the Laboratory of supramolecular biomaterials and chemistry group at the University of Strasbourg for supplying the DXP-loaded zeolite L crystals.

With this opportunity, I would like to mention my master supervisor, Prof. Ya Zhai. You led me into the research field of magnetics/spintronics. The experience and knowledge you taught me are still helpful for my PhD research. Thank you very much for the supervising, and supporting my PhD application.

Life would be tedious to me without friends. I would like to thank my dear friends, Jingfeng, Aijie, Yi, Jabiao, Yong and Chuxi, for the company in the last four years. It was always pleasure to spend my leisure time with you. Qiao, it is a great happiness to have you in my life. I will never forget the wonderful time we spent together. When I was sick, it was also you who took care of me. Thank you very much!

Finally, I would like to express my deep gratitude to my parents and elder sister. I could not make it this far without your understanding and support. Thank you!

Bojian Xu

February 2017

Enschede, The Netherlands 


\section{ISBN: 978-90-365-4286-9}

